\title{
SUPPLY CHAIN MODELS WITH CONSIGNMENT STOCK AGREEMENT, PAYMENT SCHEMES AND DELAY-IN-PAYMENTS
}

\author{
by
}

Siraj Khalid Saleh Zahran

Master of Engineering Entrepreneurship and Innovation McMaster University, Hamilton, Canada, 2011

Bachelor of Science in Systems and Industrial Engineering King Fahd University of Petroleum and Minerals, Dhahran, Saudi Arabia, 2007

A dissertation presented to Ryerson University in partial fulfillment of the requirements for the degree of Doctor of Philosophy in the program of Mechanical and Industrial Engineering Toronto, Ontario, Canada, 2017 (C) Siraj Zahran 2017 


\section{Author's Declaration}

I hereby declare that I am the sole author of this dissertation. This is a true copy of the dissertation, including any required final revisions, as accepted by my examiners.

I authorize Ryerson University to lend this dissertation to other institutions or individuals for the purpose of scholarly research.

I further authorize Ryerson University to reproduce this dissertation by photocopying or by other

means, in total or in part, at the request of other institutions or individuals for the purpose of scholarly research.

I understand that my dissertation may be made electronically available to the public. 


\title{
Supply chain models with consignment stock agreement, payment schemes and delay-in-payments
}

\section{Doctor of Philosophy 2017}

\author{
Siraj Khalid Saleh Zahran \\ Mechanical and Industrial Engineering \\ Ryerson University
}

\begin{abstract}
Supply chain management (SCM) has shown to be a successful strategy to manage the flow of goods, materials, information and services between multiple entities in one organization or multiple businesses working together to provide final customers with final products or services with the objective of improving and enhancing the performance of the chain and maximizing its profit.
\end{abstract}

Inventory management (IM) is one element of the SCM that has shown researchers' interests as it plays a major role in increasing supply chain profits and satisfying customers. Different coordination mechanisms have been developed to improve the collaboration and the integration of supply chain players.

Consignment stock (CS) is one of the coordination mechanisms that is extensively studied by researchers to reflect its benefits, drawbacks, and the proper techniques of implementing it between two or more players in the chain. The studies of the CS still have some gaps that can be covered 
by researchers such as studying its effect in a three-level supply chain or when a delay-in-payment exists. Optimizing the number of payments or studying a three-level supply chain system with multiple suppliers and multiple buyers has not been developed. This thesis covers these gaps and considers different scenarios where a CS, a traditional policy (TP) or a combination between both of them might exist in case a system consists of three players.

The main findings are optimizing the number of payments and incorporating a delay-in-payment increase the profit of the chain. In addition, a combination of a TP between the upstream players and a CS between the downstream players has shown to be better than adopting the same policy between all players. Some results of adopting a CS by all players have shown to be very close to the best scenario which could be the best option when demands highly fluctuate. 


\section{Acknowledgements}

Getting a Ph.D. degree from Ryerson University was challenging and enthusiastic. Through my journey, which took me five years, I met many people who helped, supported and encouraged me in order to achieve my goal and get the degree. I would like to thank all those people who assisted me and made this experience easy and pleasurable for me.

I would like to start by thanking everyone in my family parents, wife, children and siblings who made my life easy and were always behind my achievements. Their support, cooperation, and encouragement made me face and get rid of my life's obstacles which had enormous impacts on my study.

I also would like to thank my supervisor Professor Mohamad Y. Jaber for his support, cooperation, knowledge, and guidance that were always helpful to me in order to complete my work and get the degree. His availability and advice to me helped me to get better in learning and understanding the area that I worked on and getting rid of all obstacles that faced me through my research.

Moreover, I would like to thank the Ministry of Education (MOE) in Saudi Arabia through the Saudi Arabian Cultural Bureau (SACB) in Canada for giving me the opportunity to get through this experience and supporting my research and study.

Toronto, January 2017 
To the most great and wonderful people in my life... My mom and dad, siblings, wife and children 


\section{Table of Contents}

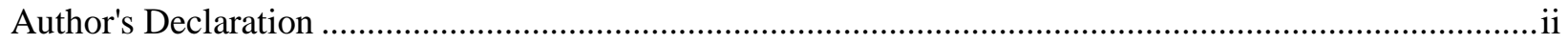

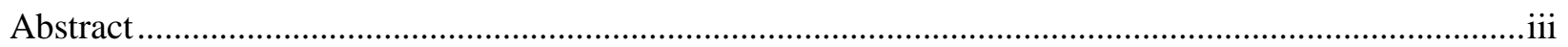

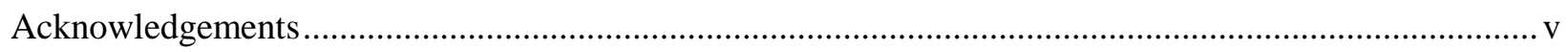

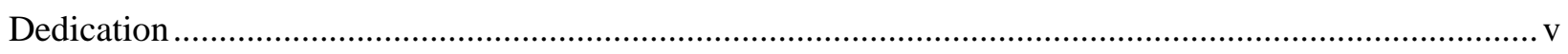

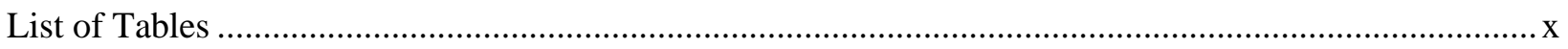

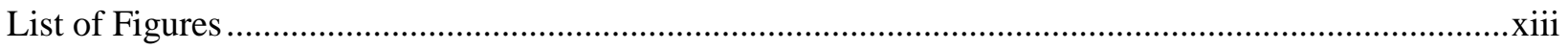

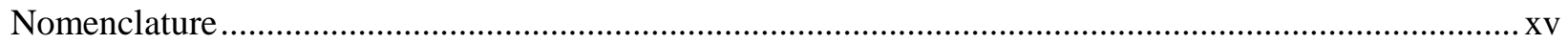

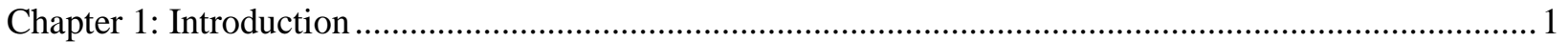

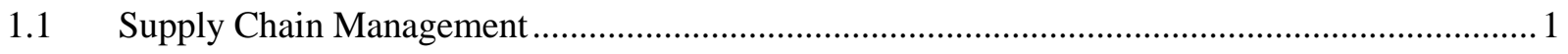

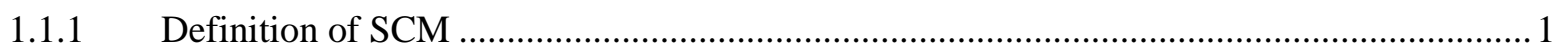

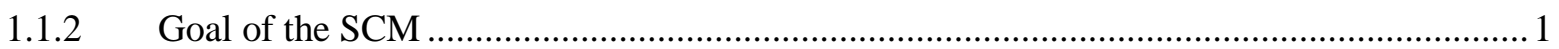

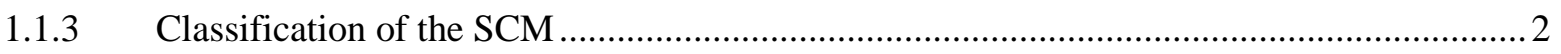

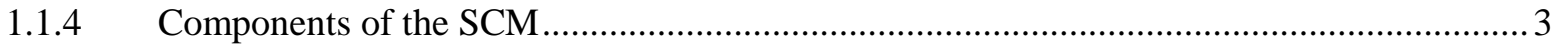

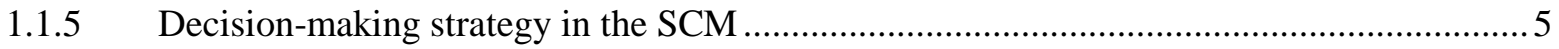

1.1.6 Centralized vs. Decentralized supply chain system ….................................................. 5

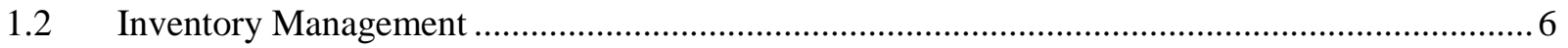

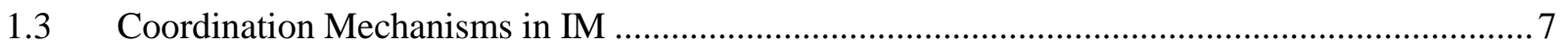

1.3.1 Vendor Managed Inventory (VMI) Systems .............................................................. 7

1.3.2 Collaborative Planning, Forecasting and Replenishment (CPFR) .................................... 8

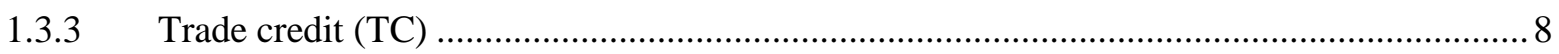

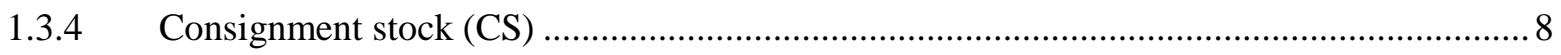

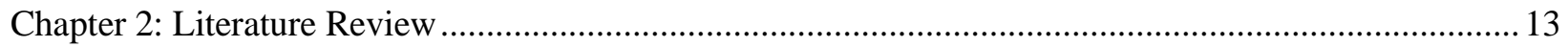

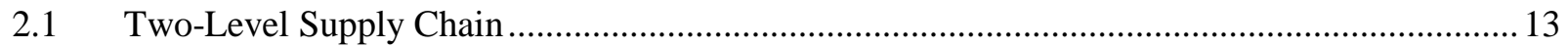

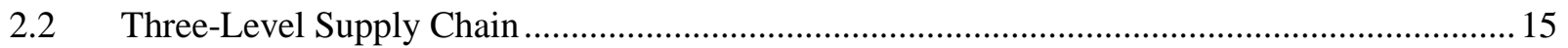

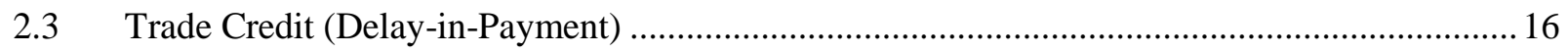

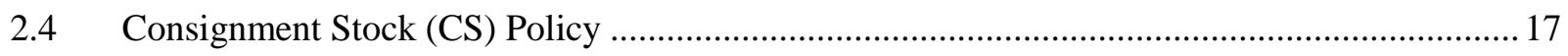

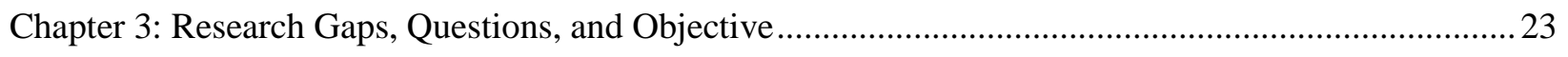

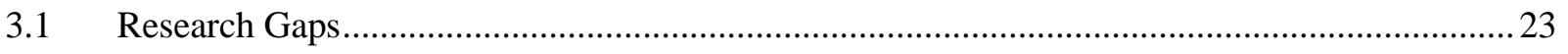

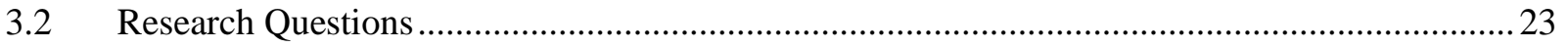

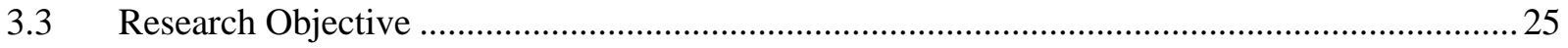

Chapter 4: Brief Description of Hill (1997), CS and TP Models.........................................................28

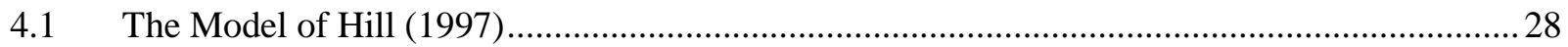




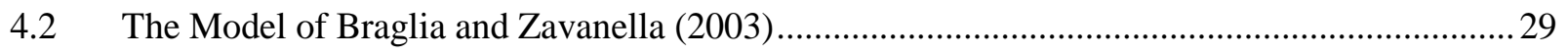

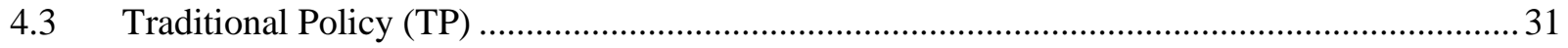

Chapter 5: Payment schemes for a two-level consignment stock supply chain system............................ 32

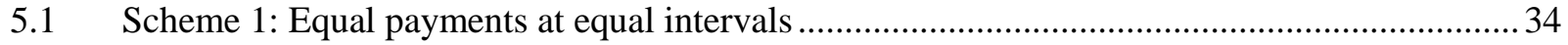

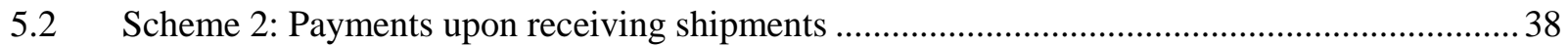

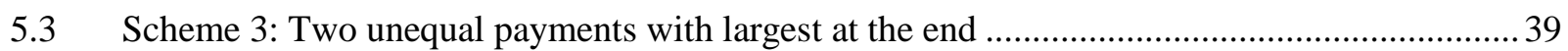

5.4 Scheme 4: Two unequal payments with smallest at the end ................................................. 41

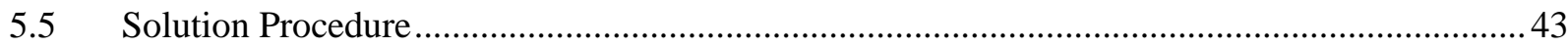

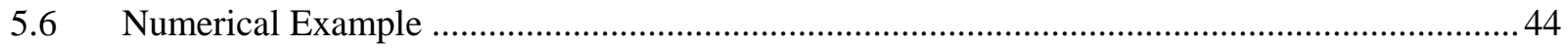

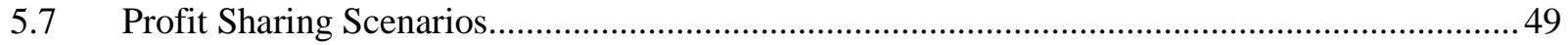

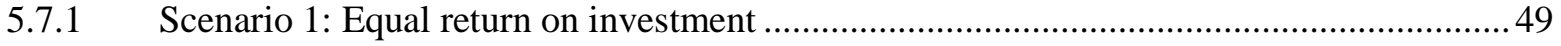

5.7.2 Scenario 2: Increase in total cost resulted from adopting the CS...................................51

5.7.3 Scenario 3: Equal distribution of the excess profit ..........................................................53

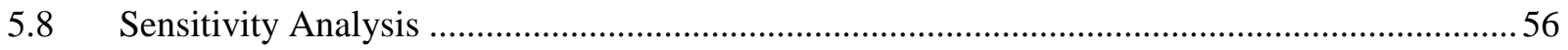

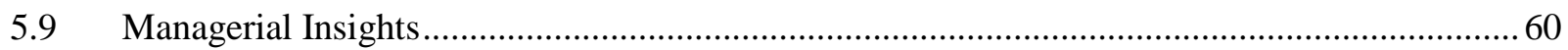

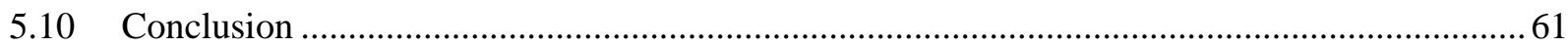

Chapter 6: The consignment stock case for a vendor and a buyer with delay-in-payments ...................... 64

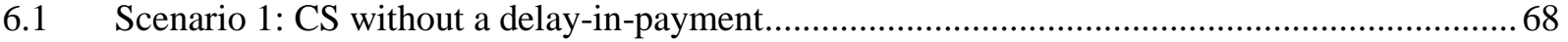

6.2 Scenario 2: CS with a delay-in-payment (Interest-free) ….................................................... 72

6.3 Scenario 3: CS with a delay-in-payment (Interest-charge) …............................................... 75

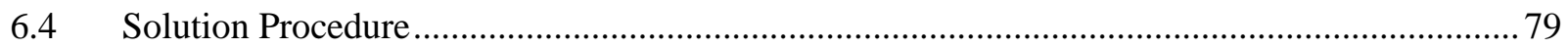

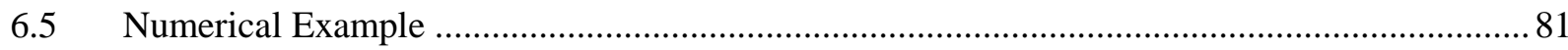

6.5.1 Comparison between the three scenarios of the CS ....................................................... 81

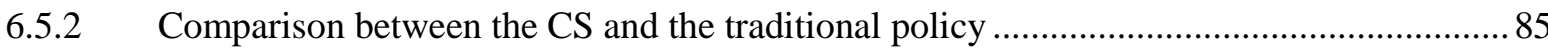

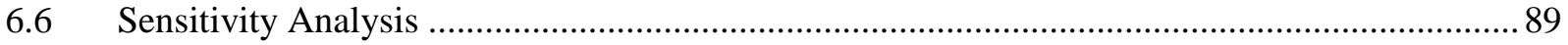

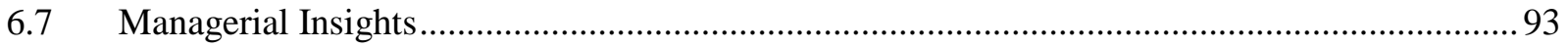

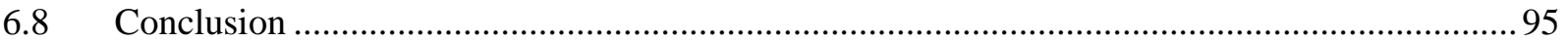

Chapter 7: Comparing different coordination scenarios in a three-level supply chain system...................97

7.1 Scenario 1: Consignment-Consignment (C-C) Scenario: ........................................................ 102

7.2 Scenario 2: No Consignment-Consignment (NC-C) Scenario:................................................ 105

7.3 Scenario 3: Consignment-No Consignment (C-NC) Scenario:................................................ 108

7.4 Scenario 4: No Consignment-No Consignment (NC-NC) Scenario:...................................... 110

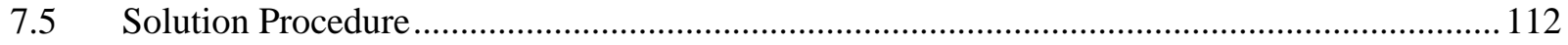




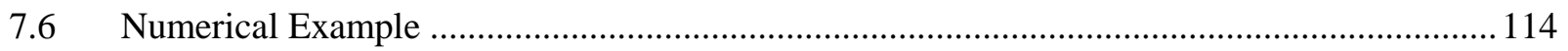

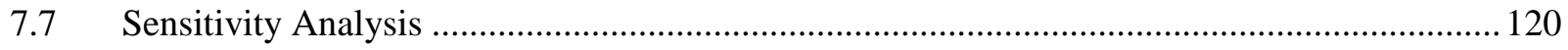

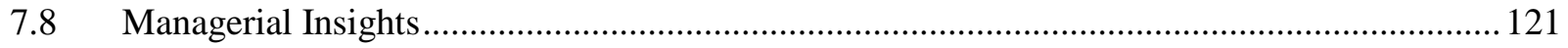

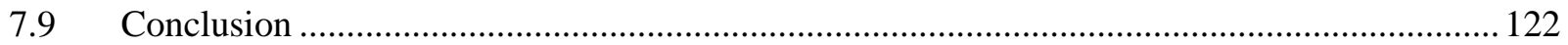

Chapter 8: Investigation of a consignment stock and a traditional inventory policy in a three-level supply chain system with multiple suppliers and multiple buyers ............................................................. 124

8.1 Brief description of the traditional policy (TP) and the consignment stock (CS) .................. 129

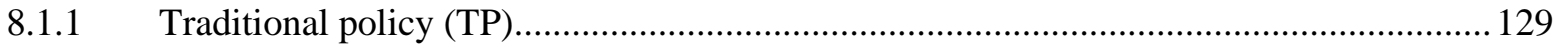

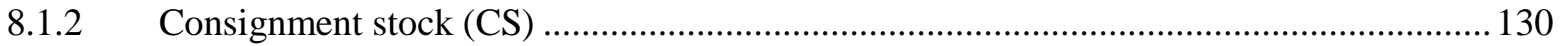

8.2 Scenario 1: No Consignment-No Consignment (NC-NC) .................................................. 131

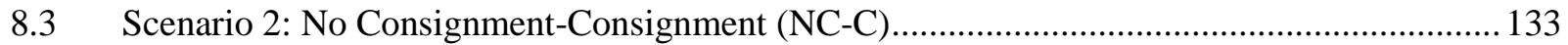

$8.4 \quad$ Scenario 3: Consignment-No Consignment (C-NC) .......................................................... 135

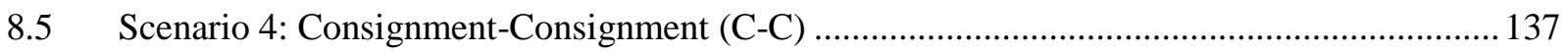

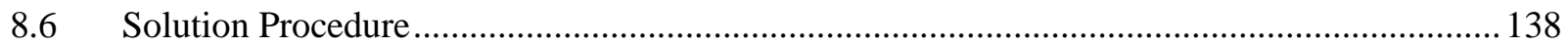

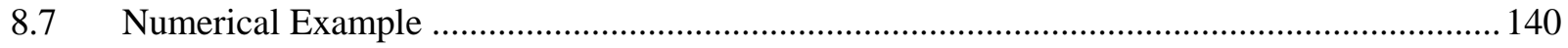

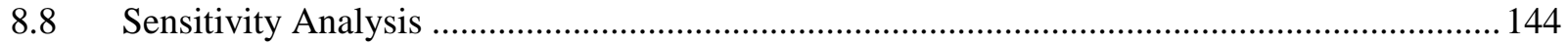

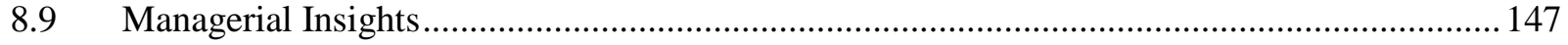

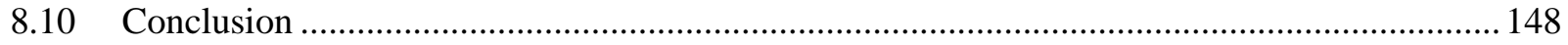

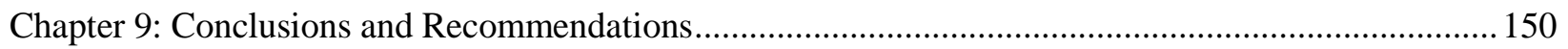

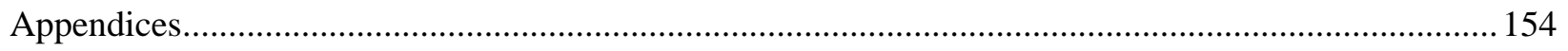

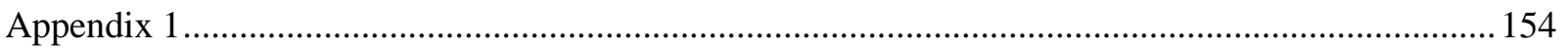

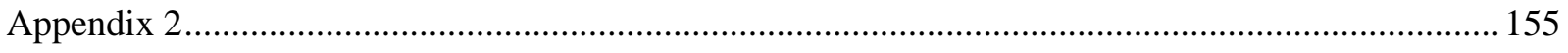

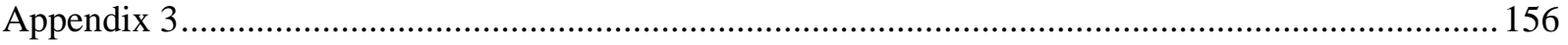

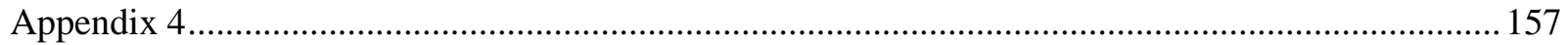

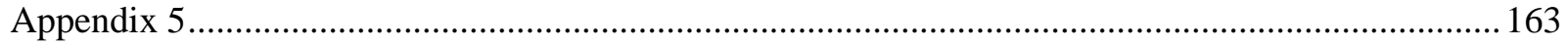

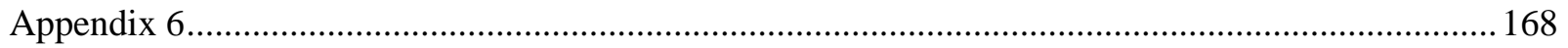

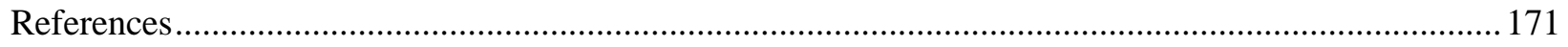




\section{List of Tables}

Table No. Description $\quad$ Page

No.

Table 1-1 References for real consignment stock contracts $\quad 10$

Table 1-2 Summary of the responsibilities for the vendor and the buyer for 12 consignment stock agreements

Table 2-1 Summary of the consignment stock literature 20

Table 5-1 Cost comparison with Braglia and Zavanella (2003) 36

Table 5-2 Values used to solve the numerical example 44

Table 5-3 Results for the optimal solution for the numerical example 45

Table 5-4 Breakdown of all variable costs and revenue in dollars for the vendor, 45 the buyer, and the system

Table 5-5 Minimum and maximum ranges, and average and standard deviation 47 values for each parameter for 2,000 numerical examples

Table 5-6 Optimal results for the total profit for B\&Z, Scheme 1+B\&Z, and 48 Scheme 1, 2, 3 and 4

Table 5-7 Deviation between the vendor's and the buyer's total profit 49

Table 5-8 Results of using the profit sharing mechanism Scenario 1

Table 5-9 Results of the non-coordinated system 52

Table 5-10 Results when using the profit sharing mechanism Scenario 2

Table 5-11 Results for Hill's model $\quad 54$

Table 5-12 Results of using the profit sharing mechanism Scenario $3 \quad 55$

Table 6-1 Samples of real consignment stock contracts $\quad 65$

Table 6-2 The distribution of the holding cost between the vendor and the buyer 67

Table 6-3 Search for Scenario 1 optimal solution $\quad 82$

Table 6-4 Search for Scenario 2 optimal solution $\quad 82$ 


\section{List of Tables Continued}

Table No. Description $\quad$ Page

No.

Table 6-5 Search for Scenario 3 optimal solution 83

Table 6-6 The optimal solutions for the three scenarios using the two approaches 83

Table 6-7 Detailed comparison between the results of the three scenarios 84

Table 6-8 Comparison between the CS and Hill (1997) models with/without the 86 delay-in-payment

Table 6-9 Minimum and maximum ranges, and average and standard deviation $\quad 87$ values for each parameter for 5,000 numerical examples

Table 6-10 Coefficients' values of the significant parameters for CS and Hill 88 models

Table 6-11 Number and percentages of examples that returned the highest system's profit for 5,000 examples

Table 7-1 The distribution of the storage-holding and the financial-holding costs 99 among the players for the four scenarios

Table 7-2 Parameter values for the numerical example 115

Table 7-3 Storage-holding costs values for the raw materials and the finished 115 items for the nine cases for each player

Table 7-4 Example of the manual search for the optimal solution for Scenario 1116 (Case 1)

Table 7-5 Optimal results for the nine cases of the numerical example

Table 7-6 Number of equations used in the calculation of the optimal quantity and the total profit

Table 7-7 Breakdown of all variable costs that affect the total system profit for all cases

Table 8-1 Example of manual search for optimal results for Scenario NC-C

(Case 1)

Table 8-2 The optimal results for Cases 1, 2 and 3 


\section{List of Tables Continued}

\begin{tabular}{llc}
\hline Table No. & Description & $\begin{array}{c}\text { Page } \\
\text { No. }\end{array}$ \\
\hline Table 8-3 & $\begin{array}{l}\text { Suppliers', Vendor's and Buyers' total profit for the optimal results } \\
\text { for Cases 1, } 2 \text { and 3 }\end{array}$ & 143 \\
Table 8-4 & $\begin{array}{l}\text { Optimal quantities and shipments for the optimal results Cases 1, 2 } \\
\text { and 3 }\end{array}$ & 144 \\
Table 8-5 & Results of the sensitivity analysis & 146 \\
Table 10-1 & Closes as stated in the consignment stock contract for companies & 154 \\
\hline
\end{tabular}




\section{List of Figures}

Figure No. Description

Figure 1-1 Supply chain management classification

Figure 1-2 Supply chain management components

Figure 1-3 Supply chain management decision-making classification $\quad 5$

Figure 3-1 Thesis structure and final objective 25

Figure 4-1 The behavior of Hill (1997) 29

Figure 4-2 The behavior of the consignment stock inventory as in Braglia and 30 Zavanella (2003)

Figure 4-3 Traditional policy considered in the thesis

Figure 5-1 The behavior of cash inventory for Scheme 1 35

Figure 5-2 The behavior of cash inventory for Scheme 2 38

Figure 5-3 The behavior of cash inventory for Scheme 3 40

Figure 5-4 The behavior of cash inventory for Scheme 4 42

Figure 5-5 Total profit while varying the ratio of $D / P$ for all payment schemes 56

Figure 5-6 Total profit while varying the ratio of $I_{b} / I_{v}$ for all payment schemes 57

Figure 5-7 Total profit while varying the ratio of $c_{c} / c_{b}$ for all payment schemes $\quad 57$

Figure 5-8 Total profit while varying the ratio of $h_{b} / h_{v}$ for all payment schemes 58

Figure 5-9 Total profit while varying the ratio of $O_{b} / S_{v}$ for all payment schemes 59

Figure 5-10 Total profit while varying the ratio of $c_{t} / c_{c}$ for all payment schemes $\quad 59$

Figure 5-11 Total profit while varying the ratio of $c_{b}^{a} / c_{v}^{a}$ for all payment schemes $\quad 60$

Figure 6-1 Behavior of the consignment stock with no delay-in-payment 69

Figure 6-2 Behavior of the consignment stock with delay-in-payment (Interest- 73 free) 


\section{List of Figures Continued}

Figure No. Description

Figure 6-3 Behavior of the consignment stock with delay-in-payment (interestcharge)

Figure 6-4 Total profit while varying $D / P$

Figure 6-5 Total profit while varying $O_{b} / S_{v}$

Figure 6-6 Total profit while varying $h_{v, f} / h_{v, s}$

Figure 6-7 Total profit while varying $h_{b, s} / h_{v, s}$

Figure 6-8 Total profit while varying $\alpha$

Figure 6-9 Total profit while varying $\beta / \alpha$

Figure 6-10 Total profit while varying $I_{b} / I_{v}$

Figure 7-1 The behavior of the Consignment - Consignment (C-C) Scenario

103

Figure 7-2 The behavior of the No Consignment - Consignment (NC-C) Scenario 106

Figure 7-3 The behavior of the Consignment - No Consignment (C-NC) Scenario 108

Figure 7-4 The behavior of the No Consignment - No Consignment (NC-NC) Scenario

Figure 8-1 General diagram for a three-level supply chain system with multiplesuppliers and multiple-buyers

Figure 8-2 General traditional policy diagram

Figure 8-3 General consignment stock diagram

Figure 10-1 Graphical representation for NC-NC Scenario 168

Figure 10-2 Graphical representation for NC-C Scenario 169

Figure 10-3 Graphical representation for C-NC Scenario 


\section{Nomenclature}

The following notations were used for Chapter 5:

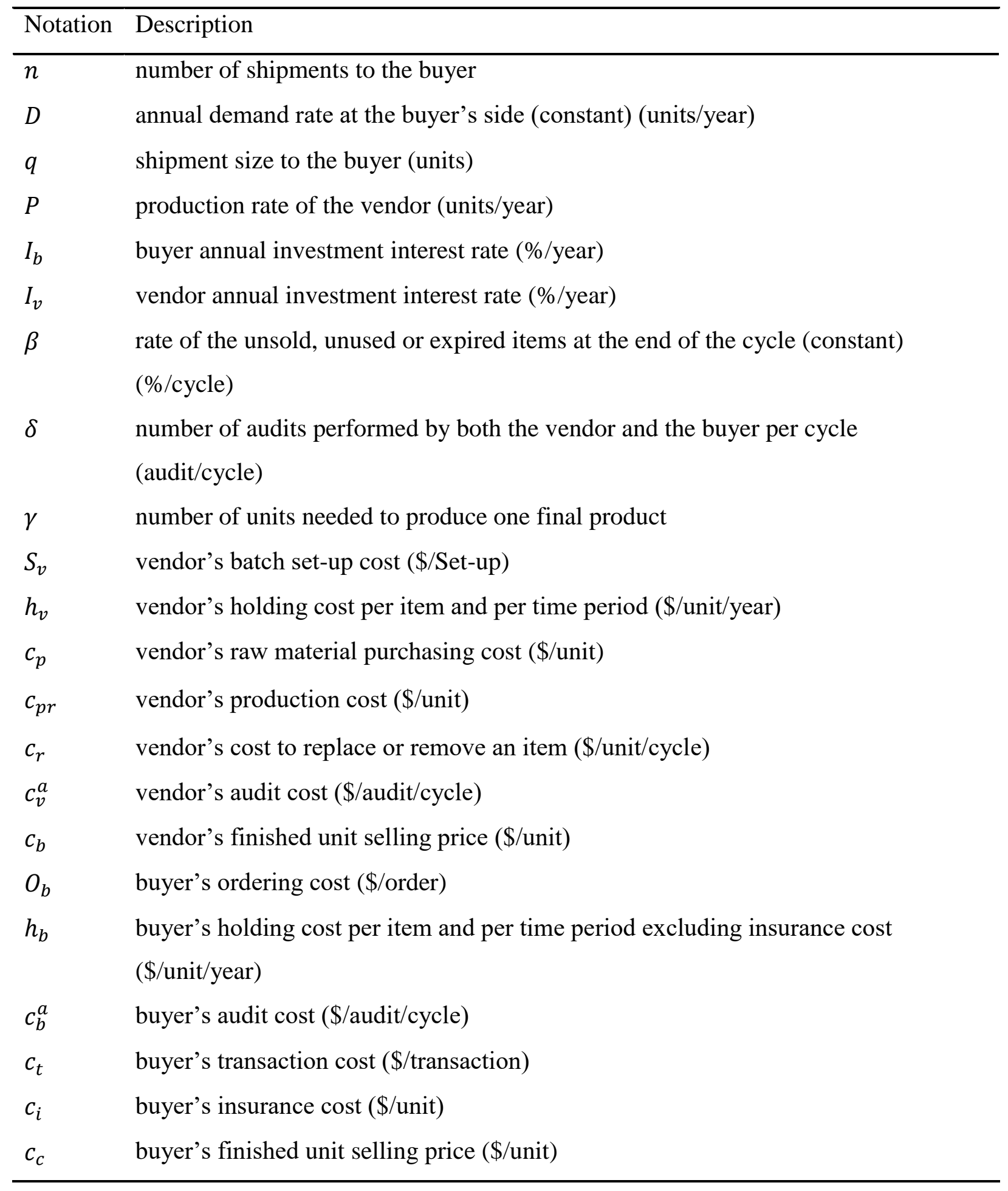


The following notations were used for Chapter $\mathbf{6}$ :

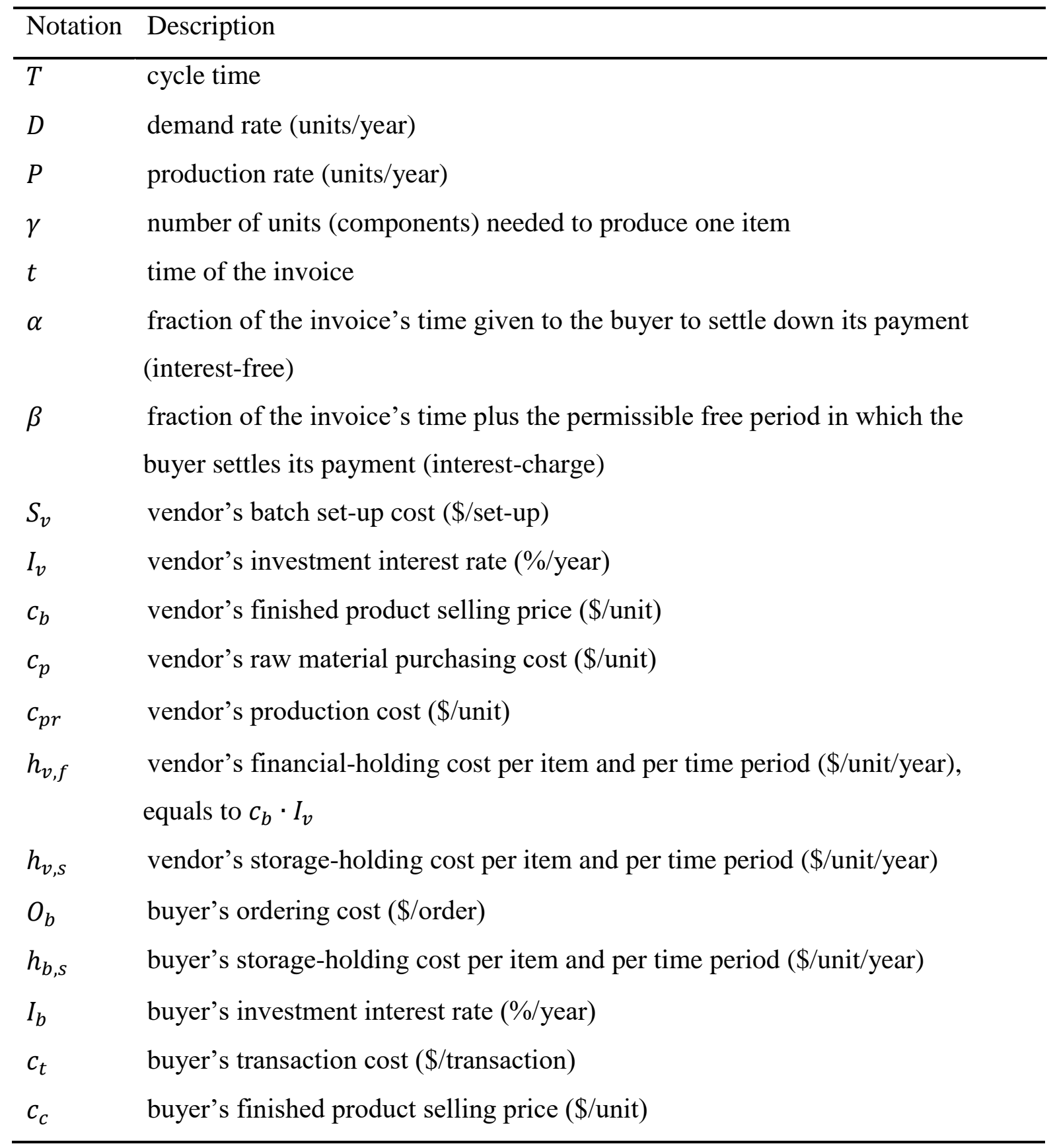


The following notations were used for the decision variables for Chapter 6:

\begin{tabular}{ll}
\hline Notation & Description \\
\hline$n_{i}$ & $\begin{array}{l}\text { number of shipments (integer) to the buyer for scenario } i, \text { where } i=1,2, \text { and } 3 \\
\text { (unit-less) }\end{array}$ \\
& $\begin{array}{l}\text { number of payments (integer) in one full cycle for scenario } i, \text { where } i=1,2 \text {, and } 3 \\
m_{i}\end{array}$ \\
$q_{i}\left(n_{i}, m_{i}\right)$ & quantity size shipped to the buyer for scenario $i$, where $i=1,2$, and 3 (units) \\
\hline
\end{tabular}

The following notations were used for Chapter 7:

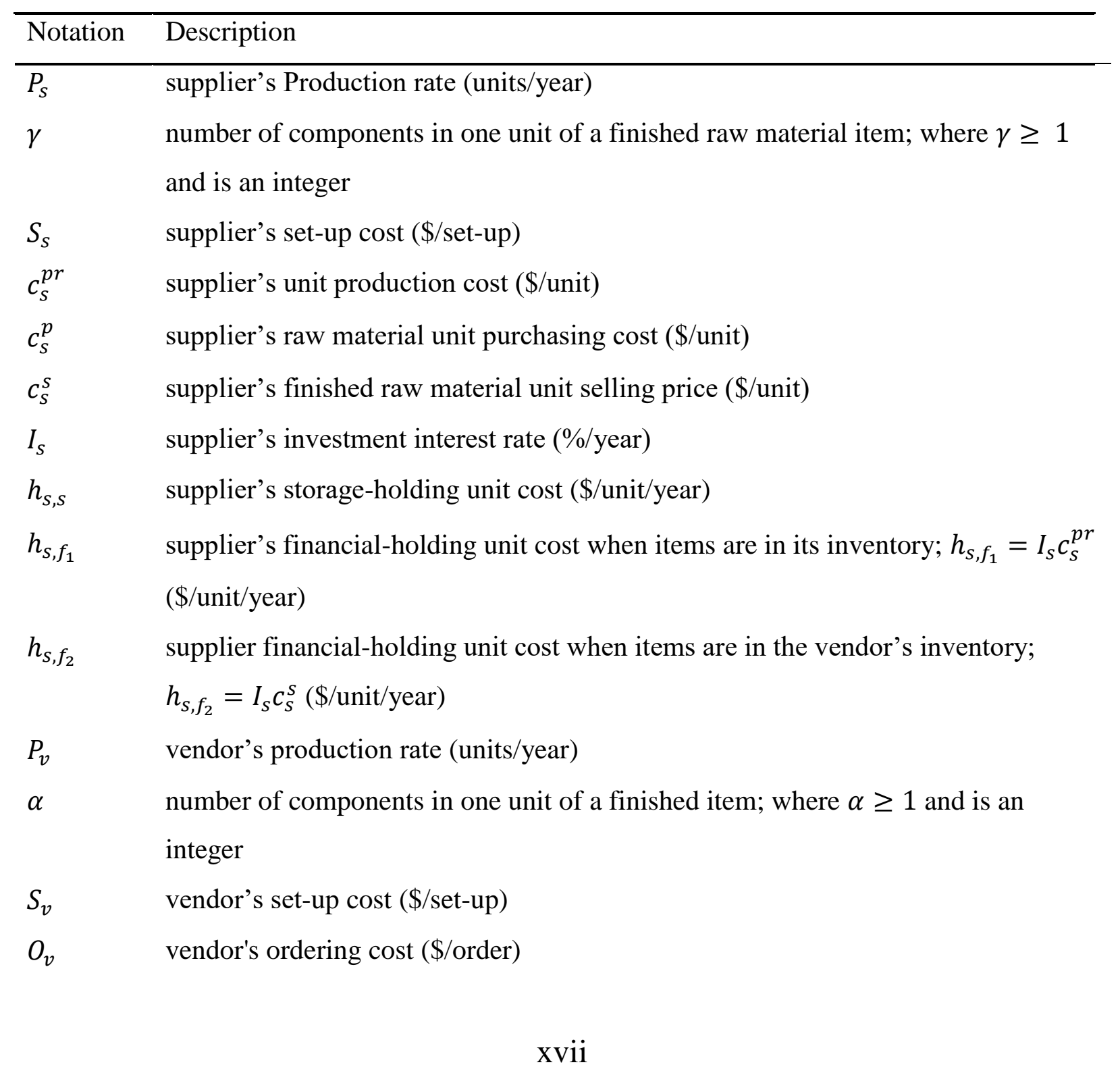




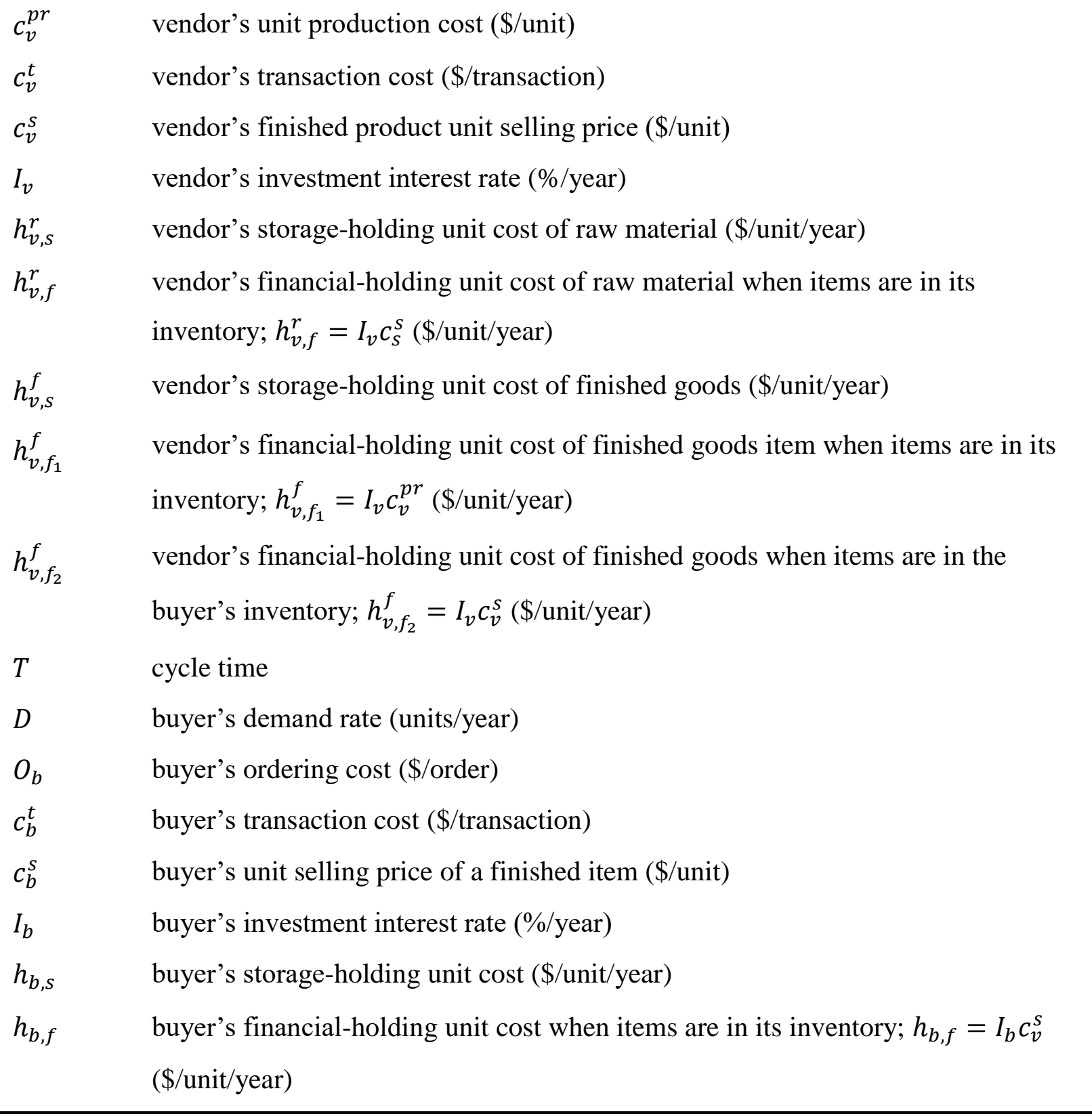

The following notations were used for the decision variables for Chapter 7 :

\begin{tabular}{ll}
\hline Notation & Description \\
\hline$n_{1}$ & number of shipments from the supplier to the vendor \\
$n_{2}$ & number of shipments from the vendor to the buyer \\
$m_{1}$ & $\begin{array}{l}\text { number of payments made by the vendor; where } m_{1}=n_{1} \text { if the traditional policy } \\
\text { (as per its assumptions) is adopted }\end{array}$
\end{tabular}



$m_{2} \quad$ number of payments made by the buyer; where $m_{2}=n_{2}$ (as per its assumptions) if the traditional policy is adopted
$q_{c} \quad$ shipment size from the vendor to the buyer when CS is adopted; where $Q_{v}=$ $n_{2} \cdot q_{c}$ is the vendor's lot size.
$q_{n c} \quad$ shipment size from the vendor to the buyer when CS is not adopted; where $Q_{v}=$ $n_{2} \cdot q_{n c}$ is the vendor's lot size.
$q_{s} \quad$ shipment size from the supplier to the vendor when CS is adopted; where $Q_{s}=$ $n_{1} \cdot q_{s}$ is the supplier's lot size.
$q_{v r} \quad$ shipment size from the supplier to the vendor when CS is not adopted; where $Q_{s}=n_{1} \cdot q_{v r}$ is the supplier's lot size.

The following notations were used for Chapter 8 :

\begin{tabular}{|c|c|}
\hline Notation & Description \\
\hline$x$ & number of suppliers, where $j=1,2,3, \ldots x$ \\
\hline$S_{S_{j}}$ & supplier $j$ batch set-up cost (\$/set-up) \\
\hline$P_{s_{j}}$ & supplier $j$ production rate (units/year) \\
\hline$\gamma_{j}$ & $\begin{array}{l}\text { number of components in one unit of a finished raw material item for supplier } j \text {; } \\
\text { where } \gamma_{j} \geq 1 \text { and integer }\end{array}$ \\
\hline$I_{s_{j}}$ & supplier $j$ investment interest rate (\%/year) \\
\hline$I_{s_{j}}^{c}$ & supplier $j$ interest charged rate for vendor (\%/year) \\
\hline$c_{s_{j}}^{p}$ & supplier $j$ raw material purchasing cost (\$/unit) \\
\hline$c_{s_{j}}^{p r}$ & supplier $j$ production cost (\$/unit) \\
\hline$c_{S_{j}}^{S}$ & $\begin{array}{l}\text { supplier } j \text { finished raw material product selling price (\$/unit); where } c_{s_{j}}^{S}= \\
\left(1+a_{s_{j}}\right)\left(c_{s_{j}}^{p}+c_{s_{j}}^{p r}\right) \text { and } a_{s_{j}} \text { is a marginal profit value for supplier } j\end{array}$ \\
\hline$h_{s_{j}}^{S}$ & supplier $j$ storage-holding cost per item (\$/unit/year) \\
\hline$h_{s_{j}}^{f_{1}}$ & $\begin{array}{l}\text { supplier } j \text { in-house financial-holding cost per item (\$/unit/year); where } h_{s_{j}}^{f_{1}}= \\
c_{s_{j}}^{p r} I_{s_{j}}\end{array}$ \\
\hline
\end{tabular}


$h_{s_{j}}^{f_{2}} \quad$ supplier $j$ out-house financial-holding cost per item (\$/unit/year); where $h_{s_{j}}^{f_{2}}=$ $c_{S_{j}}^{S} I_{S_{j}}$

$P_{v} \quad$ vendor's production rate (units/year)

$\theta_{j} \quad$ number of components needed from supplier $j$ in one unit of a finished item; where $\theta_{j} \geq 1$ and integer

$\alpha_{1, j} \quad$ fraction of the invoice's time given to the vendor by supplier $j$ to settle down its payment (interest-free)

$\beta_{1, j} \quad$ fraction of the invoice's time plus the permissible free period in which the vendor settles its payment with supplier $j$ (interest-charge)

$S_{v} \quad$ vendor's batch set-up cost (\$/set-up)

$O_{v_{j}} \quad$ vendor's ordering cost from supplier $j$ (\$/order)

$I_{v} \quad$ vendor's investment interest rate $(\% /$ year $)$

$I_{v}^{c} \quad$ vendor's interest charged rate for buyers $(\% /$ year $)$

$c_{v}^{p r} \quad$ vendor's production cost (\$/unit)

$c_{v_{j}}^{t} \quad$ vendor's transaction cost made to supplier $j$ (\$/transaction)

$c_{v}^{S} \quad$ vendor's finished product selling price $(\$ /$ unit $)$; where $c_{v}^{S}=\left(1+a_{v}\right)\left(\sum c_{s_{j}}^{S}+\right.$ $\left.c_{v}^{p r}\right)$ and $a_{v}$ is a marginal profit value for the vendor

$h_{v_{j}}^{s, r} \quad$ vendor's raw-material storage-holding cost per item received from supplier $j$ (\$/unit/year)

$h_{v_{j}}^{f, r} \quad$ vendor's raw-material financial-holding cost per item received from supplier $j$ (\$/unit/year); where $h_{v_{j}}^{f, r}=c_{s_{j}}^{s} I_{v}$

$h_{v}^{s, f} \quad$ vendor's finished-products storage-holding cost per item (\$/unit/year)

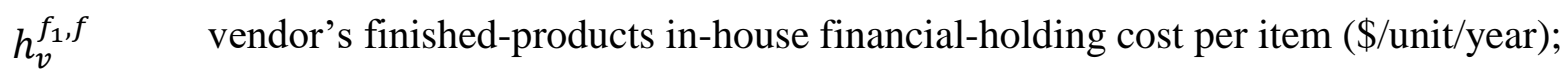
where $h_{v}^{f_{1}, f}=c_{v}^{p r} I_{v}$

$h_{v}^{f_{2}, f} \quad$ vendor's finished-products out-house financial-holding cost per item (\$/unit/year); where $h_{v}^{f_{2}, f}=c_{v}^{S} I_{v}$

$y \quad$ number of buyers, where $i=1,2,3, \ldots y$

$d_{i} \quad$ buyer $i$ demand rate (units/year) 
$\alpha_{2, i} \quad$ fraction of the invoice's time given to buyer $i$ by the vendor to settle down its payment (interest-free)

$\beta_{2, i} \quad$ fraction of the invoice's time plus the permissible free period in which buyer $i$ settles its payment with the vendor (interest-charge)

$O_{b_{i}} \quad$ buyer $i$ ordering cost (\$/order)

$I_{b_{i}} \quad$ buyer $i$ investment interest rate (\%/year)

$h_{b_{i}}^{S} \quad$ buyer $i$ storage-holding cost per item (\$/unit/year)

$h_{b_{i}}^{f} \quad$ buyer $i$ financial-holding cost per item (\$/unit/year); where $h_{b_{i}}^{f}=c_{v}^{S} I_{b_{i}}$

$c_{b_{i}}^{S} \quad$ buyer $i$ finished product selling price (\$/unit); where $c_{b_{i}}^{S}=\left(1+a_{b_{i}}\right) c_{v}^{S}$ and $a_{b_{i}}$ is a marginal profit value for buyer $i$

$c_{b_{i}}^{t} \quad$ buyer $i$ transaction cost (\$/transaction)

The following notations were used for the decision variables for Chapter 8:

\begin{tabular}{ll}
\hline Notation & Description \\
\hline$n_{1, j}$ & number of shipments from supplier $j$ to the vendor \\
$n_{2, i}$ & number of shipments from the vendor to buyer $i$ \\
$m_{1, j}$ & number of payments made by the vendor to supplier $j$ \\
$m_{2, i}$ & number of payments made by buyer $i$ to the vendor \\
$T_{z}^{*}$ & optimum cycle time for the system for scenario where z represents Scenarios 1,2, \\
& 3 and 4 \\
\hline
\end{tabular}




\section{Chapter 1: Introduction}

\subsection{Supply Chain Management}

Recently, increasing the competition in the market has forced researchers and managers to study and develop new strategies and mechanisms to improve the performance of companies and satisfy customers. Some of these studies focus on improving the internal strategy of the companies where others try to improve the external one to have a strong integrated system. One of these strategies is the Supply Chain Management (SCM), which usually helps in minimizing costs or maximizing profits of the individual organization as well as the whole supply chain system. It also helps in providing better services, quality and products to customers by integrating and linking all players in the chain.

\subsubsection{Definition of SCM}

SCM is a broad area that looks into the collaboration and the linkage between different parties in the same organization, or into two or more separate companies, working together in order to provide products or services for their customers (Stadtler, 2008). Although there are different SCM definitions in the literature, all of them cover the main ideas of this topic, which are managing information, goods and financial flow between different parties in the chain to enhance the collaboration as well as the coordination in order to optimize the system and minimize the cost (see for intense Ballou et al., 2000 and Mentzer et al., 2001).

\subsubsection{Goal of the SCM}

Competitiveness is the main goal of the SCM. It could be enhanced by reducing costs, increasing flexibility to face demand changes or variability, or providing customers with better quality products and services by improving the level of the integration and the coordination of the players in the chain (Stadtler, 2008). 


\subsubsection{Classification of the SCM}

SCM manages a range of internal and external activities of a supply chain. Researchers provided different classification of SCM. Looking at different definitions of SCM, in particular, that of Harland (1996), one can classify it into internal or external supply chain as illustrated in Figure 1-1.

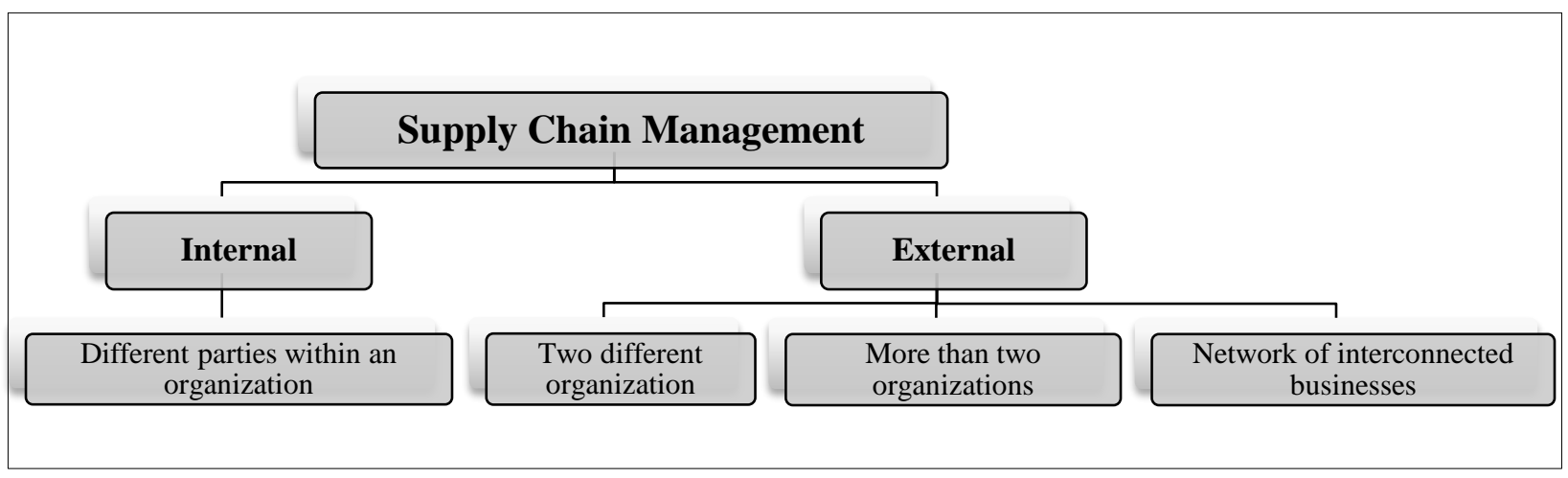

Figure 1-1. Supply chain management classification

Looking at Figure 1-1 above, one can notice that internal SCM focuses on managing the relationships between two or more parties within the same company such as manufacturing and sales departments, or among several partnering companies. Oliver and Webber (1982) were among the first to study the benefits of integrating internal functions of an organization. Also, Jones and Riley (1985) studied how managing the internal supply chain efficiently, by planning and controlling their inventories and activities as an integrated single entity, can be beneficial for an organization. It could be achieved by identifying the best location of their inventories and developing standard operation procedures. Moreover, Stevens (1989) studied the effect of developing an integrated supply chain and showed how managing the internal supply chain and working as a single entity could help in enhancing business performance.

On the other hand, external SCM looks into the collaboration, the coordination, and the integration of more than one company or organization. It enhances the relationship between two or more different organizations (i.e., supplier and vendor or supplier, vendor and retailer), or a network of interconnected businesses that are involved in providing a product or services to end customers (i.e., multi-suppliers, a vendor and multi-customers). 
Different studies have been developed for a two-level supply chain. For example, Lee and Whang (2000), and Lee et al. (2000) studied the benefits of sharing information between two parties in a supply chain and focused on finding the minimum amount of information to be shared between the partners, which could enhance coordination, integration, and system performance. Moreover, Jaber and Osman (2006) studied the benefits of the coordination between the supplier and the retailer with delay-in-payments and profit sharing on the player and the supply chain.

Other studies that considered a three-level supply chain system. For example, Munson and Rosenblatt (2001) studied the benefits of quantity discounts and showed that quantity discounts would reduce its costs. Ding and Chen (2008) studied the benefits of coordinating and profit sharing with product return on the supply chain performance. Jaber et al. (2006), as another example, studied two different mechanisms for sharing profits and showed that a mechanism where each player gets an equal return per dollar was the best.

For a network of players, Cheng and Wu (2005) studied the impact of sharing inventory information in a single vendor and multiple buyers system and found that inventory level and the expected costs decrease as sharing information increase. Furthermore, Jaber and Goyal (2008) studied the coordination of a centralized system of multiple suppliers, a single vendor, and multiple buyers and showed that the total cost of the chain either would remain the same as before the coordination or would decrease because of it. Their model was later extended to a four-level supply chain by including multiple tier-2 suppliers with multiple components and subcomponents (Jaber and Goyal, 2009).

\subsubsection{Components of the SCM}

There are two main components that help in achieving the goals of the SCM, which are "the integration of a network of organizations and the coordination of information, material, and financial flows." (Stadtler, 2008, p.11). Figure 1-2 (next page) is a schematic diagram of SCM components (level I) and subcomponents (level II). 


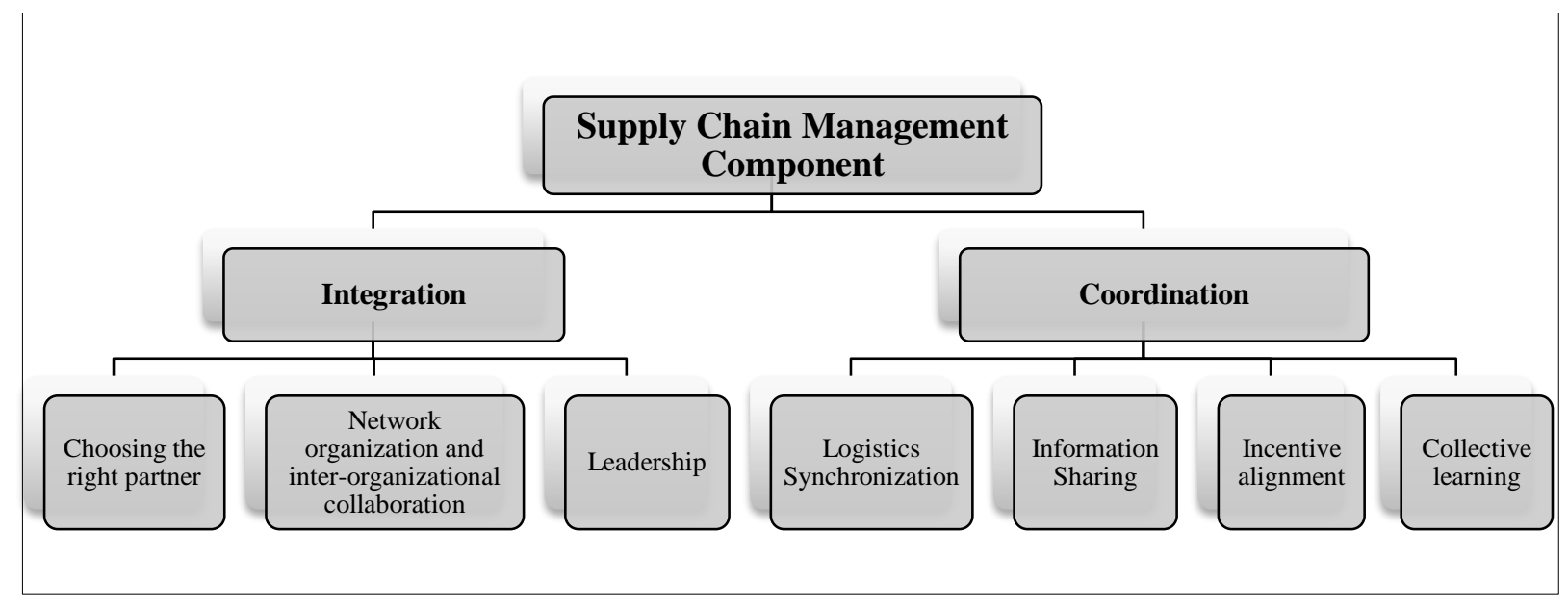

Figure 1-2. Supply chain management components

Stadtler (2008) suggested considering three aspects for a better integration and improving collaboration between different firms. These aspects are: (1) choosing the right partners that help achieve firm's goals instead of just focusing on the one that helps reduce its costs, (2) managing information, materials, and the financial flows among the players of a chain in order to increase the chain's profit through coordination, and (3) identifying the chain leader to facilitate the decision making process.

The second component of SCM is coordination, which Simatupang et al. (2002) devided into four main modes: Logistics Synchronization, Information Sharing, Incentive alignment, and Collective learning. The first mode, logistics synchronization, improves the performance and efficiency of a supply chain by recognizing the changes in customers demand, inventory management, facility layout and transportation between the players of the supply chain, reducing the forecasting errors and satisfying the customers, lowering the cost of the inventory, and improving the availability of the product (Lambert et al., 1998). The second mode, information sharing, focuses on sharing the necessary and important data between downstream and upper players. The third mode, incentive alignment, shows how one player in the supply chain is rewarded or penalized for the decision it makes. The collective learning, the fourth and last mode, considers transferring useful knowledge that one player has to the other parties in a supply chain as part of ongoing improvements to enhance the performance (Simatupang et al., 2002). 


\subsubsection{Decision-making strategy in the SCM}

Figure 1-3 classifies decision-making in a supply chain into temporal or functional.

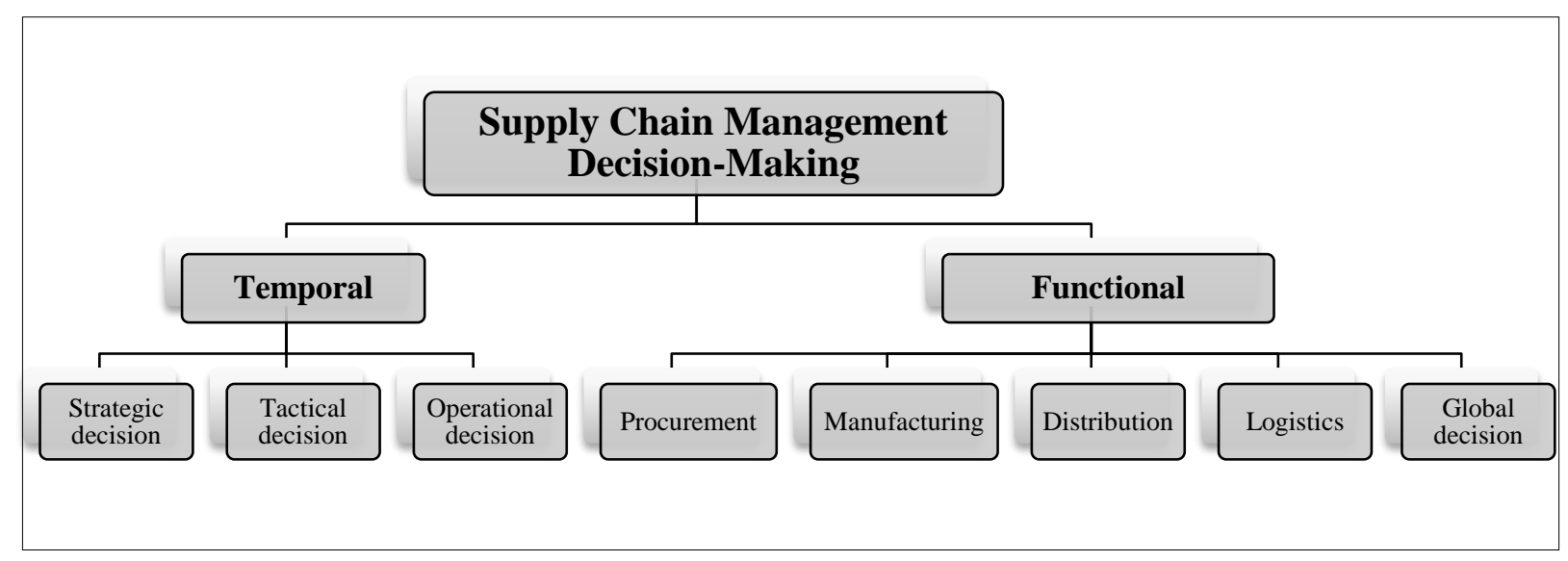

Figure 1-3. Supply chain management decision-making classification

Temporal decision-making is either strategic, tactical, or operational. Functional decision-making includes procurement, manufacturing, distribution, and logistics. Sometimes, the scope of a decision covers several functional area (global decision). Procurement decisions focus on selecting suppliers, setting appropriate inventory management techniques, determining direct/indirect deliveries from suppliers, and finding the optimal procurement policy. Manufacturing decisions focus on selecting the best location for a plant, planning and allocating capacity, setting manufacturing strategy, and optimizing the utilization of resources. Distribution decisions focus on choosing the type of the distribution facility, location, and customers to be serviced by the facility, and determining the optimal replenishment and distribution strategies. Logistics decisions focus on selecting the best transportation modes throughout a network, the appropriate port for importing or exporting products, and the optimal transportation strategy to be adopted.

\subsubsection{Centralized vs. Decentralized supply chain system}

A supply chain system can either be centralized or decentralized. A centralized supply chain is the one in which a group of people from partnering companies collectively make a decision, and evaluate and manage activities that optimize the performance of a supply chain (e.g., Fredrickson, 1986; Li and Wang, 2007). On the other hand, in a decentralized supply chain system, each member of the supply chain acts and works independently to optimize its individual system 
performance (Li and Wang, 2007). Several models for centralized and decentralized supply chain are available in the literature.

The joint economic lot size problem (JELSP), developed by Goyal (1977) and first enhanced by Banerjee (1986) and Goyal (1988), is commonly used the literature as a centralized decisionmaking tool for determining the order quantities and the number of shipments that minimize the total cost of a supply chain (e.g., Jaber and Zolfaghari, 2008; Glock, 2012). Although coordination has been shown to reduce the total cost of a supply chain, it comes at a cost to some members. Trade credit; e.g., quantity discounts, delay in payments, volume discounts, profit/savings sharing, etc., has been practiced to compensate and entice members to accept coordination (e.g., Jaber and Zolfaghari, 2008; Glock, 2012).

\subsection{Inventory Management}

Inventory management (IM) is one aspect that affects the total cost or profit of the supply chain. Richardson (1995) estimated that the inventory carrying costs in a supply chain to be between $25 \%$ and $55 \%$ of its total cost, which corroborates the claims of other studies that inventory costs can be as high as $50 \%$ of the total logistics costs (Jaber and Zolfaghari, 2008). Therefore, managing inventories by coordinating shipments among players in a supply chain has been shown to substantially reduce supply chain costs, increase the profitability of supply chain players and satisfy customers.

Researchers have developed different IM models to solve two main issues: transportation and warehousing (Williams and Tokar, 2008). Optimal inventory level helps reduce costs and increase profits. It also helps organizations to meet customer needs and minimize the out-of-stock situation (http://en.wikipedia.org/wiki/Inventory). The economic order quantity (EOQ) model, developed by Harris (1913), finds the quantity that minimizes the total cost, which is the sum of holding and setup costs. Taft (1918) modified the EOQ model by considering finite rather than an instantaneous

production rate. His model came to be known as the economic production quantity (EPQ) model. These models have been used by many researchers as base models to develop more realistic ones by relaxing some and adding other assumptions (e.g., Braglia and Zavanella, 2003; Jaber, 2007; Jaber and Zolfaghari, 2008; Glock, 2012). The plethora of papers on inventory management just goes to show the importance of the topic to academicians and practitioners. 
It is important, therefore, to give a brief review of the coordination mechanisms used to manage inventory in supply chains.

\subsection{Coordination Mechanisms in IM}

There are different mechanisms for coordinating activities in supply chains to increase customer satisfaction and supply chain profitability. Some of those mechanisms are vendor managed inventory systems (VMI), collaborative planning, forecasting and replenishment (CPFR), and consignment (Ryu, 2006). Offering trade credit has been shown to enhance a supply chain system and to go hand-in-hand with coordination.

\subsubsection{Vendor Managed Inventory (VMI) Systems}

Vendor Managed Inventory (VMI), also known as continuous replenishment or supplier-managed inventory, is a coordination mechanism that has been widely used in industry to increase customer service level and supply chain performance. Initiated by Wal-Mart and Procter \& Gamble in the 1980’s, VMI is used by many companies in the United States and Europe (Waller et al., 1999).

VMI can be defined as "A means of optimizing Supply Chain performance in which the manufacturer is responsible for maintaining the distributor's inventory levels. The manufacturer has access to the distributor's inventory data and is responsible for generating purchase orders." (vendormanagedinventory.com). It was also defined as "Inventory replenishment arrangement whereby the supplier either monitors the customer's inventory with own employees or receives stock information from the customer. The vendor then refills the stock automatically, without the customer initiating purchase orders.” (BusinessDictionary.com).

Hence, in a VMI setting, it is the responsibility of the supplier to monitor the buyers' inventory levels either physically or electronically. It is the one that makes the order quantities, shipping and timing decisions for the replenishment and informs the buyers in advance about the transactions that were taking place before receiving the shipment. (Waller et al., 1999)

Using the VMI policy helps suppliers in predicting demand and reducing the safety stock levels thus reducing their inventory costs. At the same time, buyers are guaranteed product availability 
to satisfy the customers' demand and increase the service level. Finally, VMI policy reduces transportation costs by shipping full truckload.

\subsubsection{Collaborative Planning, Forecasting and Replenishment (CPFR)}

CPFR mechanism is "a holistic approach to supply chain management and information exchange among trading partners" (J.D. Edwards, 2003, p.1). Its goal is to develop a synchronized forecast that optimizes the flow process from end to end and reducing forecasting errors. Applying CPFR in organizations increases product availability and sales while reducing the costs of inventory, transportation and logistics (Voluntary Interindustry Commerce Standards (VICS), 2004).

\subsubsection{Trade credit (TC)}

It is a common business practice that vendors provide trade credit (e.g. quantity discounts, delay in payments, price discount, etc) to increase sales and enhance cooperation with their buyers. for example, in the United States, roughly $80 \%$ of the firms deal with trade credit (Tirole, 2006), and in the United Kingdom, around $80 \%$ of business transactions end in credit (Wilson \& Summers, 2002) which increases supply chain profitability (Wu, J., et al., 2014). There are different types of trade credit in the literature such as quantity discount, price discount, delay-in-payment, etc.

A delay-in-payment, which is of interest to this thesis, can be defined as a permissible period given by a vendor to its customers to allowing them some time to settle the amounts they owe at no additional charges. Extra charges applied to balances settled after the permissible period.

\subsubsection{Consignment stock (CS)}

CS is a coordination mechanism that has been practiced in industry to increase the customer service level and the supply chain performance. It is "an innovative approach to manage inventories in which the vendor removes its inventory and maintains a stock of materials at the buyer's plant." (Battini et al., 2010b, p. 477). CS is also referred to in the literature as supplier owned inventory (SOI) (Piplani and Viswanathan, 2003) or consignment inventory (CI) (Gümüs et al., 2008). It can be adopted by players whether a decision-making strategy in a supply chain system is centralized or decentralized. A centralized supply chain is when players, represented by a team of decision makers, decide the numbers and sizes of shipments that minimize the total chain cost. On the other 
hand, a decentralized supply chain is when the decision(s) is (are) made by each player independently from the players in the chain. This usually results in conflicts among the players that requires coordinating contracts among them, for example, buyback, return policy, markdown allowance, and cost sharing are among the options (Krishnan et al., 2004). This thesis considers a centralized supply chain system when developing the mathematical models.

Many businesses, such as hospitals, clothing and furniture retailers, some gas stations, bookstores, sports equipment and musical instruments' stores, have started adopting CS agreements (Sarker, 2014). In a CS agreement, products stored at the buyer's (downstream player) belong to the vendor (upstream player). The buyer consumes or sells items in the consigned inventory and pays the vendor for the withdrawn quantities after. From reviewing different consignment stock contracts, it can be seen that the buyer is responsible for maintaining and managing the items stored in its warehouse. Also, the consignment stock has to be stored separately from the buyer's stock for easy tracking and accuracy. In addition, the buyer is responsible for providing insurance against theft, damage, and loss of items. Moreover, to avoid additional charges, received items have to be inspected for possible damage or defects before entering the buyer's warehouse. Furthermore, contrary from the VMI, it is the responsibility of the buyer to send a regular usage report to the vendor showing the quantities that have been used from inventory and to place orders to replenish its stock. In other words, in a VMI setting, the vendor is the one who decided the timing of shipments and order quantity, whereas, in a consignment stock agreement it is the buyer. (Gümüs et al., 2008) Additionally, the vendor is the one that sets and controls the selling price. The buyer's markup price is agreed upon when signing the contract. In such settings, it is the responsibility of the vendor to remove or replace any unused, unsold or expired items. which is in the favor of the buyer especially if the product is new on the market or has a short lifetime. Finally, the vendor and the buyer perform periodic reviews or audits to ensure the accuracy of the usage reports sent by the buyer to the vendor.

A CS policy has some benefits for both the vendor and the customers (buyers). One of the benefits for the vendor comes from storing items at the buyer's facility as it saves on storage costs and frees space for other products. Also, the vendor can use this policy to help in promoting or launching a new product if the customer does not want to buy it. Another benefit is that the vendor can use the consignment policy so as not to lose the buyer, especially when it is not the chain leader. For the 
customer, CS policy benefits the customer as it can save its capital for other uses since it is going to pay after using or consuming the products. Also, a consignment agreement helps the customer in avoiding shortages if the demand was uncertain or unknown, so probably no stock out will occur. (Gümüs et al., 2008) Additionally, the CS policy helps the customer in increasing or maintaining the service level as it guarantees that products are always available.

Table 1-1 identifies some of the responsibilities of the buyer and the vendor documented in actual consignment stock contracts. These responsibilities affect the costs of the players.

Table 1-1. References for real consignment stock contracts

\begin{tabular}{ll}
\hline Company name & Link \\
\hline AXIS Electronics & $\underline{\text { http://www.axiselectronics.com/images/Axis\%20Consignment_Inventory }}$ \\
BOSgreement.pdf & $\underline{\text { http://www.bos.de/fileadmin/dokumente/einkauf/D0005_E_Konsilagerve }}$ \\
Btrag_alle_Werke_EN.pdf & $\underline{\text { http://www.exceet.ch/fileadmin/exceet/downloads/contec/documents/BE2 }}$ \\
Contec Steuerungstechnik & $\underline{137 \text { Konsignationslager-Vereinbarung_EN.pdf }}$ \\
und Automation GmbH & $\underline{\text { http://www.nascosales.com/assets/downloads/cosignment_agreement.pdf }}$ \\
NASCO Distributor Sales & $\underline{\text { http://www.weplayreplay.com/ToyCA.php }}$ \\
RePlay Toys & $\underline{\text { http://www.stoneartmanufacturing.co.za/My_Homepage_Files/Download }}$ \\
STONE ART & $\underline{\text { /Consignment\%20Stock\%20Agreement\%20Revision.pdf }}$ \\
MANUFACTURING & $\underline{\text { http://www.newcastle- }}$ \\
The Newcastle upon Tyne & $\underline{\text { hospitals.org.uk/downloads/policies/Operational/ConsignmentStockPolic }}$ \\
Hospitals NHS Foundation & yandProcedure201307.pdf \\
Trust & $\underline{\text { http://www.uiowa.edu/ purchase/Shared/docs/UIHC_consignmntForm.p }}$ \\
The University of Iowa & $\underline{\text { df }}$ \\
Hospitals and Clinics & $\underline{\text { https://www.utoledo.edu/policies/administration/finance/pdfs/3364-40- }}$ \\
The University of Toledo & $\underline{06 . p d f}$ \\
Medical Center &
\end{tabular}

The responsibilities of a vendor and a buyer are:

Product ownership: The ownership of items in inventory is transferred from the vendor to the buyer after selling them to its customers or withdrawing them from the consignment inventory.

Physical loss, damage, theft, or defect: The buyer is responsible for any physical loss, damage, or defect to items while in inventory. It provides an insurance policy against unexpected costs.

Unused, unsold, or expired products: The vendor is responsible for removing and replacing unused, unsold or expired products at no cost to the buyer. 
Inspection of delivered items: It is the responsibility of the buyer to inspect items before entering its warehouse to confirm that their quality conforms to what has been agreed upon with the vendor. Periodic inventory review or audit: The vendor and the buyer conduct periodic reviews or audits of the inventory to check the quantity in stock and the storing conditions. The audit or the review is performed either weekly, monthly, quarterly, annually, or as pre-agreed.

Inventory management: It is the responsibility of the buyer to manage the CS, and to use the proper policy (e.g. the first-in-first-out (FIFO) policy) when handling, using and selling the items.

Reporting, and order initiation: The buyer is responsible for sending frequent reports to the vendor detailing the quantities used or sold from the consignment inventory, and asking for new shipments to replace the withdrawn items. Usually, the reports are sent based on the agreement between the parties; e.g. weekly or a monthly.

Product pricing: The vendor is usually the one which sets the selling price of the product. Sometimes, it is a joint pricing decision. Thus, this responsibility depends on the consignment agreement and the power of the parties involved.

Stock level management, and usage invoice: The vendor guarantees that the buyer's inventory level remains between a minimum (s) and a maximum (S), which both parties agreed on when signing the contract. Violating the limits of the inventory level subjects the vendor to penalties. The vendor is also responsible for invoicing the buyer periodically for used or sold from the consignment inventory. In addition, the vendor invoices the buyer for any damages, loss, or variation, which is caused by the buyer or its employees, that is discovered during the audit.

Payment by the due date: The vendor invoices the buyer for the items withdrawn from inventory during a given period. The buyer is usually invoiced on the first day of each month for its usage of the previous month. The vendor sets the payment date for the buyer to settle its balance (e.g., 7, 30,45 , or 60 days).

Storage space requirement: It is the buyer's responsibility to allocate space to keep the consigned inventory. 
Table 1-2. Summary of the responsibilities for the vendor and the buyer for consignment stock agreements

\begin{tabular}{lcc}
\hline \multicolumn{1}{c}{ Responsibility } & Vendor & Buyer \\
\hline Product ownership & $\checkmark$ & $\checkmark$ \\
Physical loss, damage, theft, or defect & $\checkmark$ & \\
Unused, unsold, or expired products & & $\checkmark$ \\
Inspection of delivered items & $\checkmark$ & $\checkmark$ \\
Periodic inventory review, or audit & & $\checkmark$ \\
Inventory management & & $\checkmark$ \\
Reporting, and order initiation & $\checkmark$ & $\checkmark$ \\
Product pricing & $\checkmark$ & $\checkmark$ \\
Stock level management, and usage invoice & & \\
Payment by the due date & & \\
Storage space requirement & & \\
\hline
\end{tabular}




\section{Chapter 2: Literature Review}

Supply chain management is a topic of many themes that solves some issues that the members of the chain face in today's dynamic market. Specifically, it aims at coordinating and integrating different functions in a chain to provide customers with products and services while reducing the chain's total cost and/or increasing its profitability. This chapter presents a literature review to identify the exciting research gaps and subsequently the focus and contribution of the intended thesis. Researchers in inventory management have published numerous studies that suggest methods to reducing (increasing) supply chain costs (profits). Some of them studied simple supply chain systems consisting of two levels while others studied complex structures of three or more levels (see, Jaber and Zolfaghari, 2008; Glock, 2012). Single or multiple entities (suppliers or buyers) were also considered at each level. This chapter reviews the most relevant research relating to two-level and three-level supply chain coordination, trade credit (delay-in-payments) and consignment stock policy.

\subsection{Two-Level Supply Chain}

Two-level supply chain systems have been studied extensively in the literature as being simple and illustrative models that help understand what factors affect performance. Many studies investigated two-level supply chains consisting a vendor and a buyer (or multiple buyers). Following is a brief review of such works that are relevant to this thesis.

In the area of inventory management, Harris (1913) was the first who developed the economic order quantity (EOQ) to find the optimal lot size that reduces the sum of the holding and setup costs. The work of Harris (1913) was modified by Taft (1918) by considering a finite (not instantaneous) production rate. His model came to be known as the economic production quantity (EPQ). These models have been used by many researchers as base models to develop more realistic ones by relaxing some and adding other assumptions.

Banerjee (1986) initiated a joint economic lot size problem (JELSP) in a two-level supply chain system that consists of a single vendor and a single buyer under deterministic conditions with lotfor-lot policy. This model set the foundation for the centralized coordination policy in which a decision maker (usually a group of people) determines the optimal order sizes and shipment numbers that minimize the total supply chain cost (see, Jaber and Zolfaghari, 2008; Glock, 2012). 
Banerjee divided the lead-time into three components; order transmission and production setup, production time, and delivery time. The model also considered the increase in the item's cost. Goyal (1988) developed a general JELS model. The lot-for-lot policy of Banerjee (1986) became a special case of Goyal's model.

Li et al. (1996) studied and compared two different inventory control systems, with and without cooperation between a buyer and a seller. They assumed that the demand rate is a decreasing function of the retail price and the annual inventory cost for the buyer depends on the value of the item. They also assumed that the buyer is the one who has the ordering decision and the seller uses a lot-for-lot policy. They found that the cooperation model has a higher optimal order quantity and lower wholesale price. Later, Hill (1997) developed an integrated production inventory model in a two-level supply chain system consisting of a vendor and a buyer. His model determines the production quantities and shipment schedule that minimize the average total cost per unit time. Hill assumed the vendor producing items in batches at a finite production rate and incurring a setup cost for every run. The vendor delivers items to the buyer in shipments of equal sizes who incurs an order cost with each delivery and experience a deterministic demand. The holding costs of inventory for the vendor and the buyer are time-proportional and increase when items move to downstream. In a follow-up work, Hill (1999) assumed that each batch is sent to the customer in multiple deliveries while production is running. Different cost factors were considered such as delivery costs, time-proportional stockholding cost for both the vendor and the buyer and it increases when items move to downstream, and extra fixed cost for item transfer between the parties and it is independent of batching and shipping. Hill and Omar (2006) extended the work of Hill (1999) by minimizing the long run total average cost (batch setup, delivery, holding cost for both vendor and buyer). They assumed that the buyer and the vendor collaborate to maximize their profit where a profit sharing mechanism was adopted. They also assumed holding cost increases when items move downstream. They showed that shipments are not necessarily of equal sizes. Lee (2005) developed a JELSP where a vendor orders raw materials from its supplier and uses it to produce a final product that it sells to customers. Moreover, Sajadieh et al. (2010) developed an integrated vendor-buyer system with stock dependent demand considering that the vendor delivers the final product to its buyer in equal sized batches. They found that coordination increases the profitability of the supply chain when demand is stock dependent. 
Lu (1995) studied a two-level supply chain system with a single vendor and multiple buyers assuming that the vendor knows its buyer's annual demand and order frequency from historical data. They also assumed deterministic production and demand rates, no shortages, lead-time and cost parameters do not vary over time. They assumed the setup cost to consist of a fixed and variable cost and that the buyer uses an integer-ratio purchasing policy. Abdul-Jalbar et al. (2007) developed a supply chain consisting of a vendor and two buyers. They assumed a single product, deterministic demand, finite production rate, storage-holding costs, and no shortages occur. Like other studies, they minimized the total supply chain cost.

\subsection{Three-Level Supply Chain}

Some researchers developed a more complex system consisting of a supplier (or multiple suppliers), a vendor, and a buyer (or multiple buyers). This section provides a review for some of the relevant literature.

Munson and Rosenblatt (2001) investigated the benefits from using quantity discounts on both ends and showed that it reduces the total system cost. They assumed all-unit discounts (supplier to manufacturer to retailer) as an incentive for coordination. They concluded that coordination helps in eliminating supply chain inefficiency. In a supply chain, each player looks at its benefits and maximizing its profit. Jaber et al. (2006) extended the work of Munson and Rosenblatt (2001) by considering price discounts is offered by an upstream to a downstream player with demand at the side of the buyer to be linearly increasing with discount. They investigated their model for two profit-sharing mechanisms. They maximized profits and showed a profit-sharing mechanism of an equal return on investment is beneficial to all. Jaber et al. (2010) considered a system similar to that of Munson and Rosenblatt (2001) and showed how learning in production, setups, and quality enhances the supply chain performance. They demonstrated that learning-based improvements in set-up time and rework help retailers to order frequently in smaller size and manufacturer to provide larger discounts as well as increasing the chain profits. On the other hand, they found that forgetting has negative impacts on the system as it will increase its costs.

For a system having more than a player, Yang and Wee (2001) developed a three-level supply chain that consists of a single vendor, multiple distributors and multiple retailers and showed how integrating all players in the system could result in minimizing the total cost. They also performed 
a sensitivity analysis to see how the system would be affected by changing different variables. Later, Khouja (2003) minimized the cost for a three-level supply chain system consisting of multiple firms at each level. He studied three different inventory coordination mechanisms, which are equal cycle time, the integer multipliers, and the integer powers of two multipliers. Each firm can supply two or more customers. His results showed that some mechanisms performed better than others. Ben-Daya and Al-Nassar (2008) studied two integrated production and inventory models in a supply chain system consisting of multiple suppliers, manufacturers, and retailers. They assumed that the cycle time at each stage is an integer multiple of the cycle time of the next and that shipments between stages are of equal sizes. They found that it is cheaper to send out a shipment once it is produced rather than waiting to send the whole lot. Jaber and Goyal (2008) investigated the coordination of order quantities in a three level supply chain system that consists of multiple suppliers, a vendor, and multiple buyers. They considered a centralized decisionmaking strategy. The model assumed that the suppliers send different items to the manufacturer to produce the products and sell them to the customers. The study showed that the local costs for the players remains the same or decreases as a result of the coordination. Also, savings generated from coordination are shared among the players of the chain.

Readers may also refer to Andriolo et al. (2014), Glock et al. (2014) and Buchuev et al. (2015) for recent and concise reviews of the EOQ/EPQ and supply chain models.

\subsection{Trade Credit (Delay-in-Payment)}

Another topic that has been incorporated with the JELSP to investigate its effect on the total cost (profit) of the system is the trade credit (delay-in-payment). In this area, researchers have assumed different hypothesis when developing their models. Maddah et al. (2004) studied the effect of the delay-in-payments on ordering policies considering a stochastic demand. Moreover, Jaber and Osman (2006) developed a model that coordinated the players (a supplier - a buyer) of the chain and minimized their total costs with the existence of the delay-in-payment and profit sharing. They were the first to treat the length of a delay-in-payment and the extended delay period as decision variables among others. Jaber (2007) developed a model that incorporated entropy cost into the EOQ problem with permissible delay-in-payments. In addition, Chen and Kang (2010) studied integrated inventory models allowing for delay-in-payments and variant pricing strategy. Krichen et al. (2011) developed a model that consists of a single supplier and multiple retailers considering 
a quantity discount and permissible delay-in-payments. Sarkar (2012) developed an EOQ model with delay-in-payments and time-varying deterioration rate. Another study was performed by Sarker (2012) to test the effect of the permissible delay-in-payment with stock dependent demand in the presence of imperfect production on the optimal policy of the retailer within the area of the EOQ. Furthermore, Lin et al. (2012) developed a (supplier-retailer) inventory model to find the joint optimal ordering and delivery policy by considering a trade credit and defective items. Musa and Sani (2012) studied an inventory ordering policy for deteriorating items with delay-inpayments. Some of the recent studies on this topic that considered three rather than two levels in a chain, as this is of interest to this thesis, are those of Moussawi-Haidar et al. (2014) and Aljazzar et al. (2016b). Readers may refer to Chang et al. (2008), Seifert et al. (2013), and Molamohamadi et al. (2014) for additional readings on trade credit.

\subsection{Consignment Stock (CS) Policy}

Another emerging subject that has been of interest to researchers is the CS policy, a form of the joint economic lot size problem (JELSP), which has been investigated by some researchers to study its impact on the supply chain system, its pros and cons, and its benefits as well. It is worth noting that the available studies on CS have only considered a two-level supply chain system.

Although CS has been a business practice for some time, it was not until some years ago when researchers became interested in it. Braglia and Zavanella (2003) are believed to be the first who analytically investigated the CS policy for controlling inventory. They highlighted its benefits and the areas where it could be applied. They compared their model to the model of Hill $(1997,1999)$ and they concluded that the CS is more profitable in case of demand fluctuation and helps increase service level. This model has been a base model for several investigations and extensions. Valentini and Zavanella (2003) applied the model of Braglia and Zavanella (2003) in the automotive industry and showed the pros and cons of CS policy. They found that the CS has a positive impact on the system as it increases savings, flexibility level, service level, and enhances the collaboration as well as the relationship between parties in the chain. Wang et al. (2004) showed the effect of a CS contract with revenue sharing on the performance of the system. Persona et al. (2005) developed a CS model considering the effects of obsolescence, while Tang et al. (2007) showed the benefits of this contract in an uncertain environment. Gümüş et al. (2008) studied the combined effects of CS and vendor managed inventory (VMI) in a two-level supply 
chain. Lee and Wang (2008) developed a general model for managing the level of the CI when the buyer has a limited warehouse capacity, while Huang and Chen (2009) divided the holding cost into storage and financial components. Battini et al. (2010a) developed an integrated framework that helped analysts when switching from the traditional model to the CS model. Zanoni et al. (2012) considered the effects of learning and forgetting in the production process of the vendor in a two-level supply chain that operates according to VMI with consignment agreement. Wang et al. (2012) considered deteriorating items and warehouse capacity constraint where $\mathrm{Hu}$ and $\mathrm{Li}$ (2012) studied the effect of disposing of the leftovers when there is a CS agreement with revenue sharing between the parties. Omar and Supadi (2012) studied a CS policy considering a linearly decreasing demand. Yi and Sarker (2013b) developed a CS considering a controllable lead time. In a follow-up paper, Yi and Sarker (2013a) extended their previous work and considered a buyer's space limitation. Hariga and Al-Ahmari (2013) studied a CS model by considering retail space allocation. Bazan et al. (2014) developed a VMI-CS model considering an imperfect production process with/without restoration interruptions. Braglia et al. (2014) developed a model that focuses on managing safety stock in a two-level supply chain with VMI and CS. Lee and Cho (2014) studied the benefits of sharing the stock out cost in a two-level supply chain under a deterministic and a stochastic demand. Yi and Sarker (2014) developed a consignment stock model considering a normally distributed demand, a controllable lead time and a buyer's warehouse capacity. Islam (2014) developed a consignment stock model for a seasonal product in a vendor-buyer system. Jaber et al. (2014a) modified Braglia and Zavanella (2003) by applying the laws of thermodynamics to quantify the cost of a disorder associated with the flow of items, in another paper, Jaber et al. (2014b) applied CS to manage inventory in a simple closed loop supply chain. Some researchers have investigated the model of Braglia and Zavanella (2003) for environmental issues such as carbon emissions and/or energy consumption (Zanoni et al., 2014; Bazan et al., 2015). Recently, Zanoni and Jaber (2015) developed a VMI-CS model when demand is stockdependent on the number of items on display in the buyer's store. Bylka and Górny (2015) developed a general model for the consignment stock in a two-level supply chain system and assumed that shipments do not need to be in equal sizes. Giri and Bardhan (2015) developed a consignment model considering a stock dependent demand and buyer's space limitation. In another study, Giri et al. (2015) developed a joint economic lot sizing problem (JELSP) model and 
assumed that the vendor follows a consignment stock with its buyer when the vendor's on-hand inventory reaches the maximum capacity.

In a single vendor and multiple buyers system, Liu et al. (2007) studied the effect of product obsolescence on the performance of the CS system, while Srinivas et al. (2008) used a genetic algorithm for formulating effective inventory management under a CS agreement. Zavanella and Zanoni (2009) developed a model that consists of multiple buyers, which was also considered later by Battini et al. (2010b), but with stochastic demand. Srinivas and Rao (2010) found the optimum solution using a genetic algorithm in a single vendor and multiple buyers system. Another study by Chen et al. (2010) studied a decentralized two-level supply chain system that consists of a wholesaler that distributes and sells deteriorating items to multiple non-identical retailers with the objective of maximizing profit. Sui et al. (2010) studied the effect of a reinforcement learning approach for inventory replenishment considering a VMI with consignment policy. Ben-Daya et al. (2013) showed the benefits of the VMI and CS agreement by comparing it with the other one in which the vendor and the buyers act independently of each other. Sadeghi et al. (2014) adopted the CS and studied a particle swarm optimization algorithm for a near optimal solution and used different methods for defuzzification and calibration. Mandal and Giri (2015) developed a consignment stock model considering a stock and a price dependent demand where Chen and Kan (2015) investigated a CS model for conforming and non-conforming products.

There is only one direct extension to the model of Braglia and Zavanella (2003) that studied a CS agreement in a three-level supply chain system and was made available to us by Prof. Zavanella. Surini (2011) considered a vendor who sells a product to a wholesaler, a cross-docking facility, who sells it to a buyer. He compared the traditional coordination policy with CS by considering centralized and decentralized decision-making strategies for two scenarios: (1) the vendor pays the financial-holding cost for both the wholesaler and the buyer, and (2) the vendor pays the wholesaler financial-holding cost and the latter pays the buyer. The study of Surini focused on finding the best option of paying the financial-holding part that minimizes the total cost of the system. Table 2-1 (next two pages) summarizes the CS literature that is relevant to this thesis. The papers have been classified under several headings. The mark $X$ indicates that a paper assumed/considered that heading. 
Table 2-1. Summary of the consignment stock literature

\begin{tabular}{|c|c|c|c|c|c|c|c|c|c|c|c|c|}
\hline & & & & & & & $\mathrm{h} \mathrm{Cr}$ & & & & & \\
\hline & & $\begin{array}{r}\mathrm{S} \\
\mathrm{Cha}\end{array}$ & ly & & layer & & & & & & & \\
\hline $\begin{array}{l}\text { Sr. } \\
\text { No. }\end{array}$ & Author & $\begin{array}{l}\overline{0} \\
\frac{0}{0} \\
\frac{1}{0} \\
3 \\
0\end{array}$ & 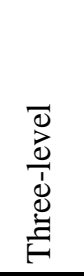 & 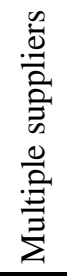 & 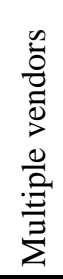 & 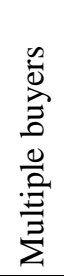 & $\begin{array}{l}\vec{\Xi} \\
\text { 馬 } \\
\tilde{\Xi}\end{array}$ & 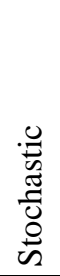 & 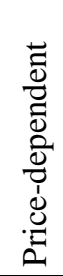 & 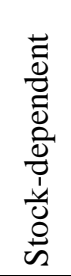 & 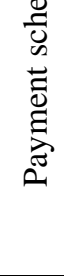 & 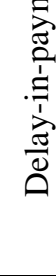 \\
\hline 1 & $\begin{array}{l}\text { Braglia and } \\
\text { Zavanella (2003) }\end{array}$ & $\mathrm{X}$ & & & & & $\mathrm{X}$ & & & & & \\
\hline 2 & $\begin{array}{l}\text { Valentini and } \\
\text { Zavanella (2003) }\end{array}$ & $\mathrm{X}$ & & & & & $X$ & & & & & \\
\hline 3 & Wang et al. (2004) & $\mathrm{X}$ & & & & & & & $\mathrm{X}$ & & & \\
\hline 4 & $\begin{array}{l}\text { Persona et al. } \\
\text { (2005) }\end{array}$ & $\mathrm{X}$ & & & & & $\mathrm{X}$ & & & & & \\
\hline 5 & Tang et al. (2007) & $\mathrm{X}$ & & & & & & $\mathrm{X}$ & & & & \\
\hline 6 & Gümüş et al. (2008) & $\mathrm{X}$ & & & & & $\mathrm{X}$ & & & & & \\
\hline 7 & $\begin{array}{l}\text { Lee and Wang } \\
\text { (2008) }\end{array}$ & $\mathrm{X}$ & & & & & $\mathrm{X}$ & & & & & \\
\hline 8 & $\begin{array}{l}\text { Huang and Chen } \\
(2009)\end{array}$ & $X$ & & & & & $\mathrm{X}$ & & & & & \\
\hline 9 & $\begin{array}{l}\text { Battini et al. } \\
(2010 a)\end{array}$ & $\mathrm{X}$ & & & & & & $\mathrm{X}$ & & & & \\
\hline 10 & Zanoni et al. (2012) & $\mathrm{X}$ & & & & & $\mathrm{X}$ & & & & & \\
\hline 11 & Wang et al. (2012) & $\mathrm{X}$ & & & & & $\mathrm{X}$ & & & & & \\
\hline 12 & $\mathrm{Hu}$ and $\mathrm{Li} \mathrm{(2012)}$ & $\mathrm{X}$ & & & & & & $\mathrm{X}$ & & & & \\
\hline 13 & $\begin{array}{l}\text { Omar and Supadi } \\
(2012)\end{array}$ & $\mathrm{X}$ & & & & & $\mathrm{X}$ & & & & & \\
\hline 14 & $\begin{array}{l}\text { Yi and Sarker } \\
(2013 \text { b) }\end{array}$ & $\mathrm{X}$ & & & & & $\mathrm{X}$ & & & & & \\
\hline 15 & $\begin{array}{l}\text { Yi and Sarker } \\
(2013 a)\end{array}$ & $\mathrm{X}$ & & & & & $\mathrm{X}$ & & & & & \\
\hline 16 & $\begin{array}{l}\text { Hariga and Al- } \\
\text { Ahmari (2013) }\end{array}$ & $\mathrm{X}$ & & & & & & & & $\mathrm{X}$ & & \\
\hline 17 & Bazan et al. (2014) & $X$ & & & & & $\mathrm{X}$ & & & & & \\
\hline 18 & $\begin{array}{l}\text { Braglia et al. } \\
\text { (2014) }\end{array}$ & $\mathrm{X}$ & & & & & $\mathrm{X}$ & & & & & \\
\hline 19 & Lee and Cho (2014) & $\mathrm{X}$ & & & & & & $\mathrm{X}$ & & & & \\
\hline 20 & $\begin{array}{l}\text { Yi and Sarker } \\
(2014)\end{array}$ & $\mathrm{X}$ & & & & & & $\mathrm{X}$ & & & & \\
\hline 21 & Islam (2014) & $\mathrm{X}$ & & & & & & $\mathrm{X}$ & & & & \\
\hline 22 & Jaber et al. (2014a) & $\mathrm{X}$ & & & & & $\mathrm{X}$ & & & & & \\
\hline 23 & Jaber et al. (2014b) & $\mathrm{X}$ & & & & & $\mathrm{X}$ & & & & & \\
\hline
\end{tabular}


Table 2-1. (continued) Summary of the consignment stock literature

\begin{tabular}{|c|c|c|c|c|c|c|c|c|c|c|c|c|}
\hline \multirow[b]{3}{*}{$\begin{array}{l}\text { Sr. } \\
\text { No. }\end{array}$} & \multirow[b]{3}{*}{ Author } & \multicolumn{11}{|c|}{ Search Criterion } \\
\hline & & \multicolumn{2}{|c|}{$\begin{array}{c}\text { Supply } \\
\text { Chain Level }\end{array}$} & \multicolumn{3}{|c|}{ Players } & \multicolumn{4}{|c|}{ Demand } & \multirow[b]{2}{*}{ 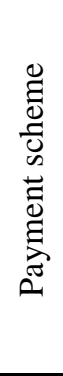 } & \multirow{2}{*}{ 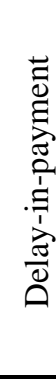 } \\
\hline & & $\begin{array}{l}\overline{0} \\
\overrightarrow{0} \\
\frac{0}{1} \\
0 \\
3 \\
\end{array}$ & 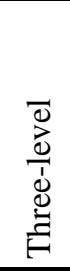 & 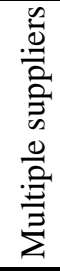 & 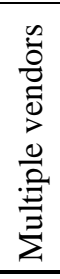 & 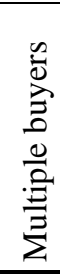 & $\begin{array}{l}\vec{\Xi} \\
\text { ज्ञ } \\
\tilde{0} \\
0\end{array}$ & 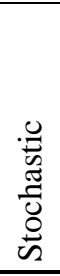 & 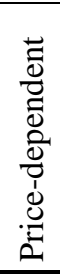 & 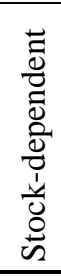 & & \\
\hline 24 & Zanoni et al. (2014) & $\mathrm{X}$ & & & & & $\mathrm{X}$ & & & & & \\
\hline 25 & Bazan et al. (2015) & $X$ & & & & & $\mathrm{X}$ & & & & & \\
\hline 26 & $\begin{array}{l}\text { Zanoni and Jaber } \\
\text { (2015) }\end{array}$ & $\mathrm{X}$ & & & & & & & & $\mathrm{X}$ & & \\
\hline 27 & $\begin{array}{l}\text { Bylka and Górny } \\
\text { (2015) }\end{array}$ & $\mathrm{X}$ & & & & & $\mathrm{X}$ & & & & & \\
\hline 28 & $\begin{array}{l}\text { Giri and Bardhan } \\
\text { (2015) }\end{array}$ & $\mathrm{X}$ & & & & & & & & $\mathrm{X}$ & & \\
\hline 29 & Giri et al. (2015) & $\mathrm{X}$ & & & & & $X$ & & & & & \\
\hline 30 & Liu et al. (2007) & $\mathrm{X}$ & & & & $\mathrm{X}$ & $\mathrm{X}$ & & & & & \\
\hline 31 & $\begin{array}{l}\text { Srinivas et al. } \\
(2008)\end{array}$ & $\mathrm{X}$ & & & & $\mathrm{X}$ & & $\mathrm{X}$ & & & & \\
\hline 32 & $\begin{array}{l}\text { Zavanella and } \\
\text { Zanoni (2009) }\end{array}$ & $\mathrm{X}$ & & & & $\mathrm{X}$ & $\mathrm{X}$ & & & & & \\
\hline 33 & $\begin{array}{l}\text { Battini et al. } \\
(2010 b)\end{array}$ & $\mathrm{X}$ & & & & $\mathrm{X}$ & & $\mathrm{X}$ & & & & \\
\hline 34 & $\begin{array}{l}\text { Srinivas and Rao } \\
(2010)\end{array}$ & $\mathrm{X}$ & & & & $\mathrm{X}$ & & $\mathrm{X}$ & & & & \\
\hline 35 & Chen et al. (2010) & $X$ & & & & $\mathrm{X}$ & & $\mathrm{X}$ & & & & \\
\hline 36 & Sui et al. (2010) & $X$ & & & & $X$ & $\mathrm{X}$ & & & & & \\
\hline 37 & $\begin{array}{l}\text { Ben-Daya et al. } \\
\text { (2013) }\end{array}$ & $\mathrm{X}$ & & & & $X$ & $\mathrm{X}$ & & & & & \\
\hline 38 & $\begin{array}{l}\text { Sadeghi et al. } \\
(2014)\end{array}$ & $\mathrm{X}$ & & & & $X$ & & $\mathrm{X}$ & & & & \\
\hline 39 & $\begin{array}{l}\text { Mandal and Giri, } \\
(2015)\end{array}$ & $\mathrm{X}$ & & & & X & & & $\mathrm{X}$ & $\mathrm{X}$ & & \\
\hline 40 & $\begin{array}{l}\text { Chen and Kan } \\
(2015)\end{array}$ & $\mathrm{X}$ & & & & $X$ & & $\mathrm{X}$ & & & & \\
\hline 41 & Surini (2011) & & $\mathrm{X}$ & & & & $\mathrm{X}$ & & & & & \\
\hline
\end{tabular}

The literature review shows that most of the studies considered two-level supply chain systems operating under a traditional policy (EOQ or JELSP). Some have considered three-level systems. Most of the studies considered a single entity (supplier, vendor or buyer) at each level, where some considered multiple entities. Some of the studies in the literature considered trade credit 
agreements (e.g., delay-in-payment, a profit sharing, price discount, quantity discounts) between the players in a supply chain to entice coordination and mitigate unexpected costs or losses.

In the area of CS, the literature review also shows that most of the researchers focused their research on a two-level supply chain system considering different mechanisms or criteria such as profit sharing, limited warehouse capacity, controllable lead-time, and obsolescence or deteriorating items. Few researchers tried to extend the two-level supply chain system with a single vendor and a single buyer to a model that considers a vendor deals with two or multiple buyers.

To the best of the author's knowledge and from the literature, no study yet has investigated the effect of different payment schemes and the delay-in-payments on the total profit of the CS system. None of the CS studies have considered a three-level supply chain system that consists of multiple suppliers (produce raw materials), a vendor (stores raw materials and produces finished items), and multiple buyers. Therefore, this thesis addresses all of these limitations in the CS literature. 


\section{Chapter 3: Research Gaps, Questions, and Objective}

Researchers in supply chain management have been investigating how to develop mathematical or simulation models that reflect reality of businesses and environments. The objectives of these models are centered on improving supply chain performance that, mainly, revolves around reducing costs and increasing profits.

Under the umbrella of the SCM, CS is one of the IM areas that has been practiced by businesses for some time and showed to have many benefits for the supply chain system when adopted by the players in a chain. The CS literature shows different gaps that have not been addressed and questions that have not been answered yet. Table 2-1 lists the CS published studies (marked by X mark) and the gaps that can be filled (empty white cells), which are mentioned in bullet points in Section 3.1 .

\subsection{Research Gaps}

$>$ The number of payments that maximizes the total profit of the system when a CS agreement is adopted still has not been investigated.

Researchers have not investigated the effect of delay-in-payments on the total profit of the supply chain system when a CS agreement is adopted.

> Investigating the CS agreement in a three-level supply chain system that consists of a supplier, a vendor, and a buyer is still a gap in the CS literature.

Investigating the CS agreement in a complex structure of a three-level supply chain system that consists of multiple suppliers (produces raw materials), a vendor (stores raw materials and produces finished products), and multiple buyers, has not been done.

\subsection{Research Questions}

Researchers have considered the impractical situation in which a payment is made upon the withdrawal of items from the consignment inventory. This an impractical case. More realistic is for the seller to bill the buyer periodically where the buyer makes a payment upon receiving an invoice from the seller. The question becomes how frequent the seller should invoice the buyer? How does this affect the profitability of the system? 
What are the possible and practical payment/invoice schemes that can be followed by players when they adopt a CS agreement? Which of the considered schemes would maximize the total profit of the system? How this is different from the payment scheme adopted for a traditional policy?

Researchers have investigated the effect of the delay-in-payment on the traditional supply chain system, but not when a CS is adopted between the players in a supply chain. This is surprising given that it has been shown to be commonly practiced. Since a payment in CS already delayed until the items are withdrawn from inventory, how a delay-in-payment can be incorporated into a CS agreement? Also, how does the delay-in-payment affect the total profit of the system? Is it better for the upstream player to offer the downstream player a permissible period to settle its balance?

The literature shows that the CS policy has been investigated in a two-level supply chain for various situations. The literature shows that there is no study that investigated a CS policy in the context of a three-level supply chain. This thesis does that and answers the question: How does a three-level supply chain system behave when a CS agreement is adopted by all or just adjacent players in the chain? What are the possible coordination scenarios that can be followed among the players in a three-level supply chain system? What is the best scenario among these scenarios and for what conditions?

The developed three-level supply chain model has been extended to consider multiple suppliers and multiple players at their respective levels. The extended model accounts for delay-in-payments and a scheme of equal-sized payments at equal time intervals. Similar to the above questions, what are the possible coordination scenarios that can take form between different levels of the chain? What is the scenario that maximizes the system's profit? Is it better, or not, for the supply chain to offer the downstream players permissible delay periods to settle their accounts?

In general, what are the situations in which a CS agreement performs better than a traditional policy? What are the benefits for each player in the supply chain when a CS agreement is adopted? Is the best option (scenario) for the system is the best for all players? How can a losing player be compensated? 


\subsection{Research Objective}

The primary objective of the thesis is to develop a more realistic model that consists of multiple suppliers who produce semi-finished products (raw materials or components/parts) and ship them to a vendor that stores them and later uses them to produce a finished product, ships the finished items of the product to multiple buyers. The model also accounts, when applicable, for a scheme of equal-sized payments at equal intervals with/without delay-in-payments. To achieve the final objective outlined herein, four different models are developed as explained in Figure 3-1.

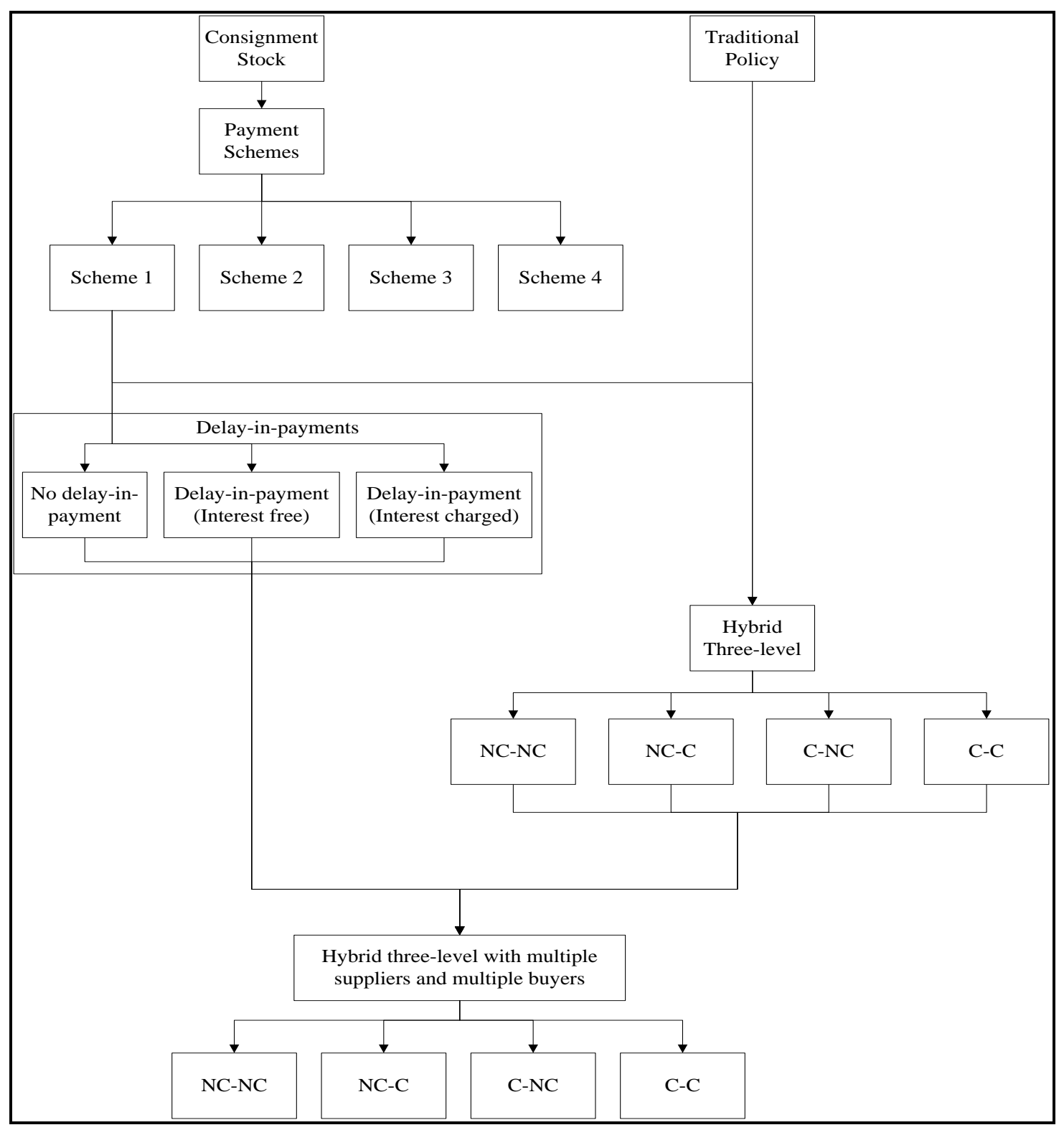

Figure 3-1. Thesis structure and final objective 
A significant effort has been made by the author to address the identified research gaps and to answer the above questions. Several inventory models have been developed and optimized using different optimization methods such as linear and non-linear programming and differential calculus. The effects of several input parameters on the developed models have been investigated to observe their influence on the performance of supply chain system. The research presented in this thesis is:

Chapter 4: This chapter briefly describes the CS model of Braglia and Zavenella (2003), which is considered as the first to investigate the CS in a two-level supply chain. In addition, the model of Hill (1997), a form of traditional policy (TP), is presented as it is used later in the thesis to compare it with the CS model(s) that is(are) developed in this thesis. This chapter, therefore, provides the background to the models developed in this thesis. Finally, an overview of TP that is used in Chapters 7 and 8.

Chapter 5: Four payment schemes are investigated in a CS two-level supply chain context is presented. Considering a payment scheme, rather than paying for items upon withdrawal, brings additional costs and revenues that arise for interest earned or charged. These costs must be factored into the developed cost/profit functions. This requires setting number of payments in a cycle as a decision variable. Also, three profit sharing mechanisms are developed and a comparison with the model of Hill (1997) is made. (Note that the context of this chapter is published online and the reference to the paper is: Zahran, S. K., Jaber, M. Y., Zanoni, S., \& Zavanella, L. E. (2015). Payment schemes for a two-level consignment stock supply chain system. Computers \& Industrial Engineering, 87, 491-505.)

Chapter 6: This chapter develops a CS case for a vendor and a buyer with a permissible delay-inpayment. Three different scenarios, with and without delay-in-payments, have been developed and a comparison between them is performed to test the effect of delay-inpayments when a CS agreement is in place. Two approaches that return the same results are used to solve the problem. The developed CS scenarios are compared with the TP of Hill (1997) after modifying its mathematics. (Note that the context of this chapter is published online and the reference to the paper is: Zahran, S. K., Jaber, M. 
Y., \& Zanoni, S. (2016). The consignment stock case for a vendor and a buyer with delay-in-payments. Computers \& Industrial Engineering, 98, 333-349.)

Chapter 7: The literature shows that there is no study that investigated the CS policy, adopted by all or only by adjacent players, in the context of a three-level supply chain system consisting of a supplier (produces raw material), a vendor (stores raw material and produces finished products) and a buyer. This chapter is believed to be the first to address the limitation in the CS literature and develops a three-level supply chain system. When having more than two levels, it may be possible that a CS policy is not employed by all players, only between adjacent ones. As a result, four possible coordination scenarios are considered. It also considers an equal payment scheme between adjacent players in the supply chain. (Note that the context of this chapter is published online and the reference to the paper is: Zahran, S. K., Jaber, M. Y., \& Zanoni, S. (2016). Comparing different coordination scenarios in a three-level supply chain system. International Journal of Production Research, 1-21.)

Chapter 8: Different from the works in the literature and developed in the previous chapters, the main objective of this chapter is to develop a three-level supply chain system that consists of multiple suppliers, a vendor, and multiple buyers. Similar to Chapter 7, four scenarios are developed considering the possibility of adopting different agreements (a CS and no-CS) at the same time between the adjacent parties in the chain. All suppliers or buyers are assumed to have the same type of agreement. This chapter investigated the above scenarios with and without the possibility of offering a delayin-payment by the upstream players and the optimal number of equal payments has been found. (Note that the context of this chapter has been submitted for review in Applied Mathematical Modelling.)

In the end, Chapter 9 represents an overall conclusion and some recommendation for future research works. 


\section{Chapter 4: Brief Description of Hill (1997), CS and TP Models}

The main focus of this research is to study the CS policy for different situations, test the effects of different factors and parameters on the performance of a supply chain system and to compare it with a traditional coordination policy, i.e. Hill (1997), from the literature to get some insights and recommendations. This chapter starts with a brief description of the model of Hill (1997) and the base CS model of Braglia and Zavanella (2003). It also provides a brief description of another traditional policy that is used later in Chapters 7 and 8 that compare four scenarios that represent combinations of consignment and traditional coordination agreements.

\subsection{The Model of Hill (1997)}

Hill (1997) developed a general integrated production-inventory policy for a two-level supply chain system (a vendor and a buyer). The objective of this model is to minimize the average total cost for the system. In his model, it was assumed that the vendor produces a single product, in batches, at a finite rate and ships it to the buyer that faces a deterministic demand rate. The vendor is assumed to incur a fixed setup and a storage-holding for items in its inventory costs. At the same time, the buyer incurs a fixed ordering and a storage-holding for items in its inventory costs. It was also assumed that the vendor delays its production and starts only at the time it produces exactly the quantity needed by the buyer for the first shipment. This reduces the inventory level to zero after the first shipment is dispatched. Hill (1997) assumed that the proportional increase in the size of two successive shipments can be between 1 and $P / D$. The focus of this research is on the equal size shipment strategy as it is close to the CS policy. Figure 4-1 (next page) shows the behavior of Hill (1997).

The average total cost for Hill (1997) when the sizes of the shipments are equal as expressed in his paper is

$$
C=\left(A_{1}+n A_{2}\right) \frac{D}{n q}+h_{1}\left(\frac{D q}{P}+\frac{(P-D) n q}{2 P}\right)+\left(h_{2}-h_{1}\right) \frac{q}{2}
$$

Where $A_{1}$ and $A_{2}$ represent a fixed setup and ordering costs, respectively, and $h_{1}$ and $h_{2}$ are for the storage-holding costs for the vendor and the buyer, respectively. The optimal quantity that minimizes the average total cost for the system is 


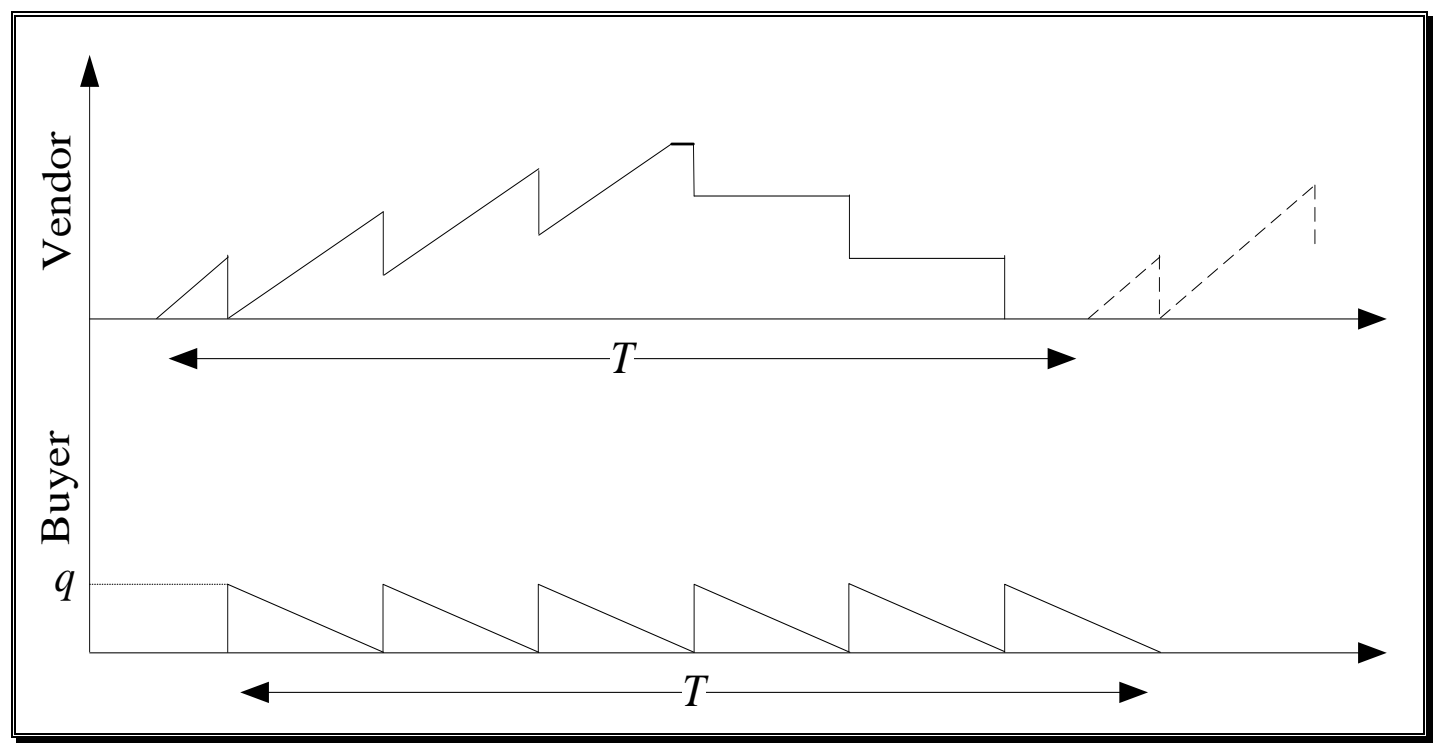

Figure 4-1. The behavior of Hill (1997)

$$
q^{*}=\sqrt{\frac{\left(A_{1}+n A_{2}\right) \frac{D}{n}}{h_{1}\left(\frac{D}{P}+\frac{(P-D) n}{2 P}\right)+\frac{\left(h_{2}-h_{1}\right)}{2}}}
$$

and a minimum total cost that is equal to

$$
C\left(q^{*}\right)=2 \sqrt{\left(A_{1}+n A_{2}\right) \frac{D}{n} / h_{1}\left(\frac{D}{P}+\frac{(P-D) n}{2 P}\right)+\frac{\left(h_{2}-h_{1}\right)}{2}}
$$

\subsection{The Model of Braglia and Zavanella (2003)}

Although CS has been practiced for some period, Braglia and Zavanella (2003) were the first to analytically investigate the CS for controlling inventories in a two-level (a vendor and a buyer) supply chain system. They also highlighted its benefits and the areas where it could be applied.

In their paper, the authors assumed that there is a consignment agreement between the players, and the vendor sells a single product to the buyer. The inventory behavior is described in their paper and can be summarized as follow. The vendor produces $n q$ units in a cycle of length $T$, which is the same for the buyer. The vendor ships $q$ units every $q / P$ units of time, where $n q / P<T=$ $n q / D$. The buyer's inventory reaches a maximum of $q(n-(n-1) D / P)$ in which is depleted by 
the buyer over the period $T-n q / P$, which is the period over which the vendor does not produce the product. The behavior of the CS as in Braglia and Zavanella (2003) is seen in Figure 4-2.

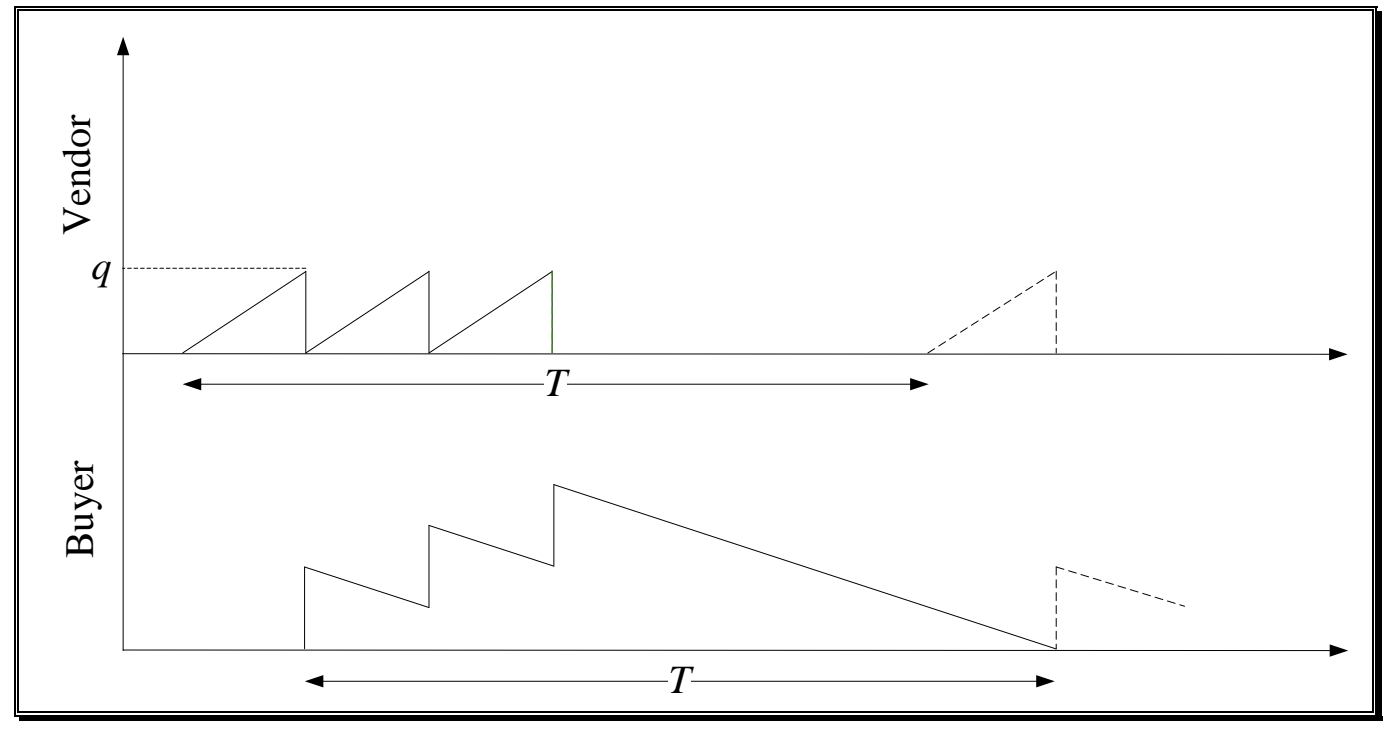

Figure 4-2. The behavior of the consignment stock inventory as in Braglia and Zavanella (2003)

The total cost per unit of time (annual) for the vendor, $C_{v}$, and the buyer, $C_{b}$, are given from Braglia and Zavanella (2003), respectively, as

$$
\begin{aligned}
& C_{v}=C_{v}^{1}+C_{v}^{2}=\frac{A_{v} D}{n q}+h_{1} \frac{q D}{2 P} \\
& C_{b}=C_{b}^{1}+C_{b}^{2}=\frac{A_{b} D}{q}+h_{2} \frac{q}{2}\left(n-(n-1) \frac{D}{P}\right)
\end{aligned}
$$

Where $C_{i}^{1}$ and $C_{i}^{2}$ are, respectively, the setup/order and holding per unit of time (annual) costs for player $i(i=v, b)$. The total supply chain cost is then written as

$$
C_{S}=C_{v}+C_{b}=\left(A_{v}+n A_{b}\right) \frac{D}{n q}+h_{2}\left(\frac{q D}{P}+n q \frac{P-D}{2 P}\right)-\left(h_{2}-h_{1}\right) \frac{q D}{2 P}
$$

where $h_{1}=\left(h_{v, f}+h_{v, s}\right)$ and $h_{2}=\left(h_{v, f}+h_{b}\right)$

$h_{v, f}$ is the financial part of the holding cost where $h_{v, s}$ is the stocking part of the holding cost. 


\subsection{Traditional Policy (TP)}

One more TP that is close to the one developed by Hill (1997) and is used in the thesis assumes that the vendor produces a single item in batches, at a finite rate, and ships equal size shipments $(q)$ to the buyer that faces a constant demand in multiple transport operations while the production is running. The vendor stops its production when it reaches $n q$, and continues shipping the quantity needed by the buyer every $q / D$ unit of time. The behavior of this policy is illustrated in Figure 4-3.

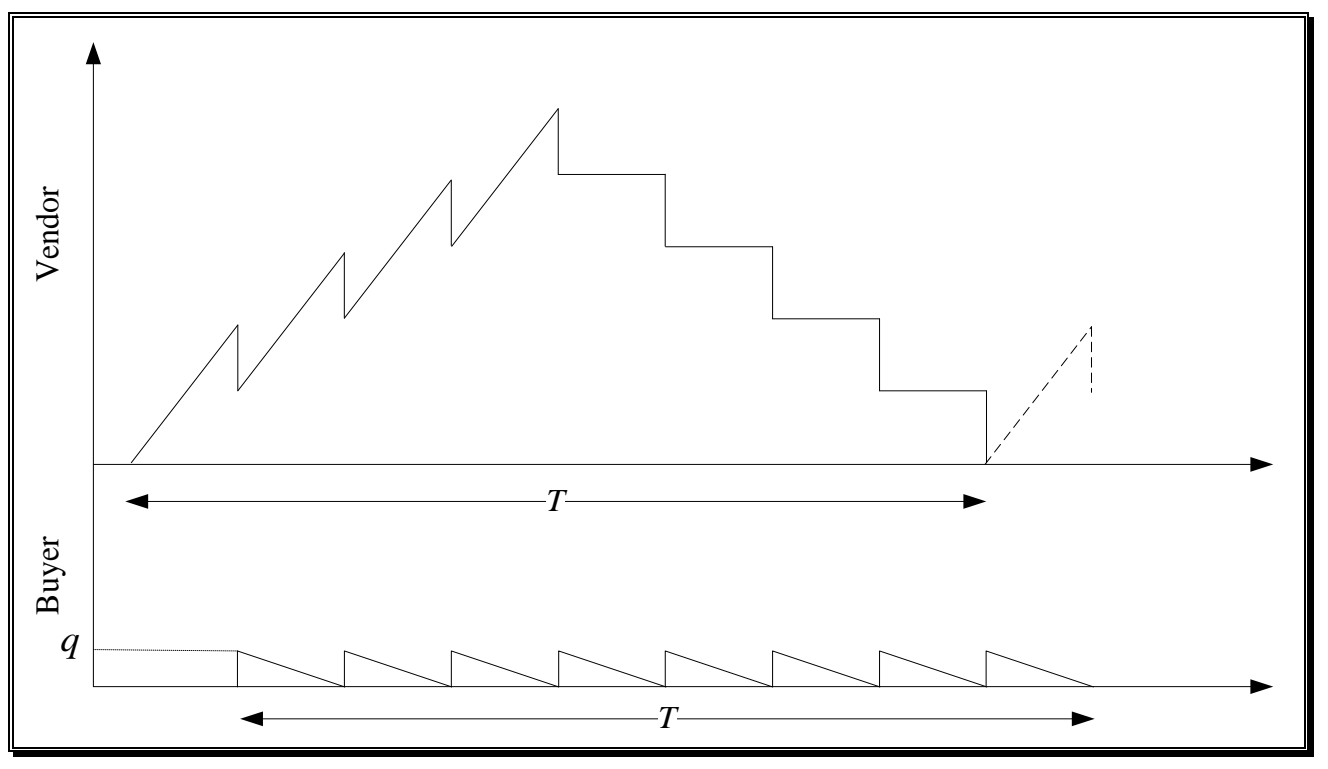

Figure 4-3. Traditional policy considered in the thesis 


\section{Chapter 5: Payment schemes for a two-level consignment stock supply chain system}

CS mechanism has been practiced by many businesses as it brings many benefits to collaborating parties. For example, it improves service levels for collaborating firms and customer satisfaction by making products available, and increases profits.

The main goal of this chapter is to investigate some real aspects of CS contracts. It modifies the work of Braglia and Zavanella (2003) by accounting for some related costs that arise from adopting a billing/payment scheme between the vendor and the buyer in a two-level supply chain system. The relevant studies on CS in the literature implied that the payments are made by the downstream player to the upstream player upon the withdrawal of items from inventory. This assumption is not realistic and not applied and/or practiced in a real-world setting as shown from the surveyed contracts in Table 1-1. From another perspective, it significantly increases the cost as there are fees associated with each money transaction/transfer and negatively affect the profitability of the supply chain. In this regard, this chapter focuses on these two main issues (other related costs and different payment schemes) that have not been addressed in the literature, and unlike earlier works it uses a profit maximization approach. It also studies the effects of different payment schemes and associated cost factors on the total profit of a two-level (vendor-buyer) supply chain system for a single product operating according to a consignment stock coordination agreement. The behavior of inventory as shown in Braglia and Zavanella (2003) is provided in Section 4.2 of this thesis.

For the model of Braglia and Zavanella (2003), who adopted a cost minimization approach and did not consider any payment scheme that illustrates how the buyer pays the vendor for the supplied items, four different payment schemes are proposed, which are:

- Scheme 1: Equal payments at equal intervals.

- Scheme 2: Payments upon receiving shipments.

- Scheme 3: Two unequal payments with largest at the end.

- Scheme 4: Two unequal payments with smallest at the end.

The following assumptions were considered when developing the models: 
1- The demand is known and constant.

2- The production rate of the vendor is greater than the demand rate of the buyer to avoid shortages.

3- The value of the product increases as it moves forward in a supply chain. The holding cost also increases.

4- The buyer does not pay for the product until it is used or sold.

5- A single finished product is considered.

6- The vendor and the buyer have a common cycle time.

7- Lead time is neglected (zero) to simplify the model and show the exact effects of the payment schemes.

8- The buyer has to insure the consigned products and the insurance cost for the consigned products is excluded from the holding cost.

9- The vendor has to remove or replace any unused, unsold or expired products instantaneously.

10- The vendor and the buyer perform a periodic audit review.

The notations that are used in the mathematical modeling for this chapter as follow.

$n \quad$ number of shipments to the buyer

$D \quad$ annual demand rate at the buyer's side (constant) (units/year)

$q \quad$ shipment size to the buyer (units)

$P \quad$ production rate of the vendor (units/year)

$I_{b} \quad$ buyer annual investment interest rate (\%/year)

$I_{v} \quad$ vendor annual investment interest rate (\%/year)

$\beta \quad$ rate of the unsold, unused or expired items at the end of the cycle (constant) (\%/cycle)

$\delta \quad$ number of audits performed by both the vendor and the buyer per cycle (audit/cycle)

$\gamma \quad$ number of units needed to produce one final product 


\begin{tabular}{|c|c|}
\hline$S_{v}$ & vendor's batch set-up cost (\$/Set-up) \\
\hline$h_{v}$ & vendor's holding cost per item and per time period (\$/unit/year) \\
\hline$c_{p}$ & vendor's raw material purchasing cost (\$/unit) \\
\hline$c_{p r}$ & vendor's production cost (\$/unit) \\
\hline$c_{r}$ & vendor's cost to replace or remove an item (\$/unit/cycle) \\
\hline$c_{v}^{a}$ & vendor's audit cost (\$/audit/cycle) \\
\hline$c_{b}$ & vendor's finished unit selling price (\$/unit) \\
\hline$O_{b}$ & buyer's ordering cost (\$/order) \\
\hline$h_{b}$ & $\begin{array}{l}\text { buyer's holding cost per item and per time period excluding insurance cost } \\
\text { (\$/unit/year) }\end{array}$ \\
\hline$c_{b}^{a}$ & buyer's audit cost (\$/audit/cycle) \\
\hline$c_{t}$ & buyer's transaction cost ( $\$ /$ transaction) \\
\hline$c_{i}$ & buyer's insurance cost (\$/unit) \\
\hline$c_{c}$ & buyer's finished unit selling price (\$/unit) \\
\hline
\end{tabular}

The rest of this chapter is organized as follows: Section 5.1 is for Scheme 1, Section 5.2 presents Scheme 2, Section 5.3 is for Scheme 3, Section 5.4 is for Scheme 4, Section 5.5 presents a solution procedure, Section 5.6 is for the numerical example, Section 5.7 presents different profit sharing scenarios, Section 5.8 performs a sensitivity analysis, Section 5.9 represents managerial insights and the chapter ends with Section 5.10, which is for summary, conclusions and future work.

\subsection{Scheme 1: Equal payments at equal intervals}

The first model studies the effect of $m$ equal payments on the total profit of the system. Braglia and Zavanella (2003) assumed that the buyer pays the vendor continuously (on a daily basis) for the used or sold items, which is impractical. Scheme 1 assumes that the vendor sends an invoice to the buyer every $T / m$ period of time for its consumption in that period. Accordingly, the buyer makes $m$ payments of $c_{b} D T / m$ each to the vendor at the same time it receives the invoice. The buyer's revenue in a cycle is $c_{c} D T$ which is invested at an interest rate $I_{b}$ to generate additional profits. On the other hand, there is a delay in investing $c_{b} n q$ (the vendor's revenue in a cycle) at an interest rate $I_{v}$ until the payment is made by the buyer, suggesting a loss of opportunity of not investing the money for a period of time. The behavior of the cash inventory for the vendor and 
the buyer is shown in Figure 5-1. The grey triangles in Figure 5-1, which illustrate the inventory behavior at the buyer's side, represent the cumulative sales made by the buyer from directly selling the items to customers. At the vendor side, the area under the dashed line shows the cumulative investment for the payments which is made by the vendor in one cycle. The grey rectangles also represent the vendor's tied up capital in inventory stocked at the buyer's warehouse. All the calculations are made for a one full cycle to be consistent.

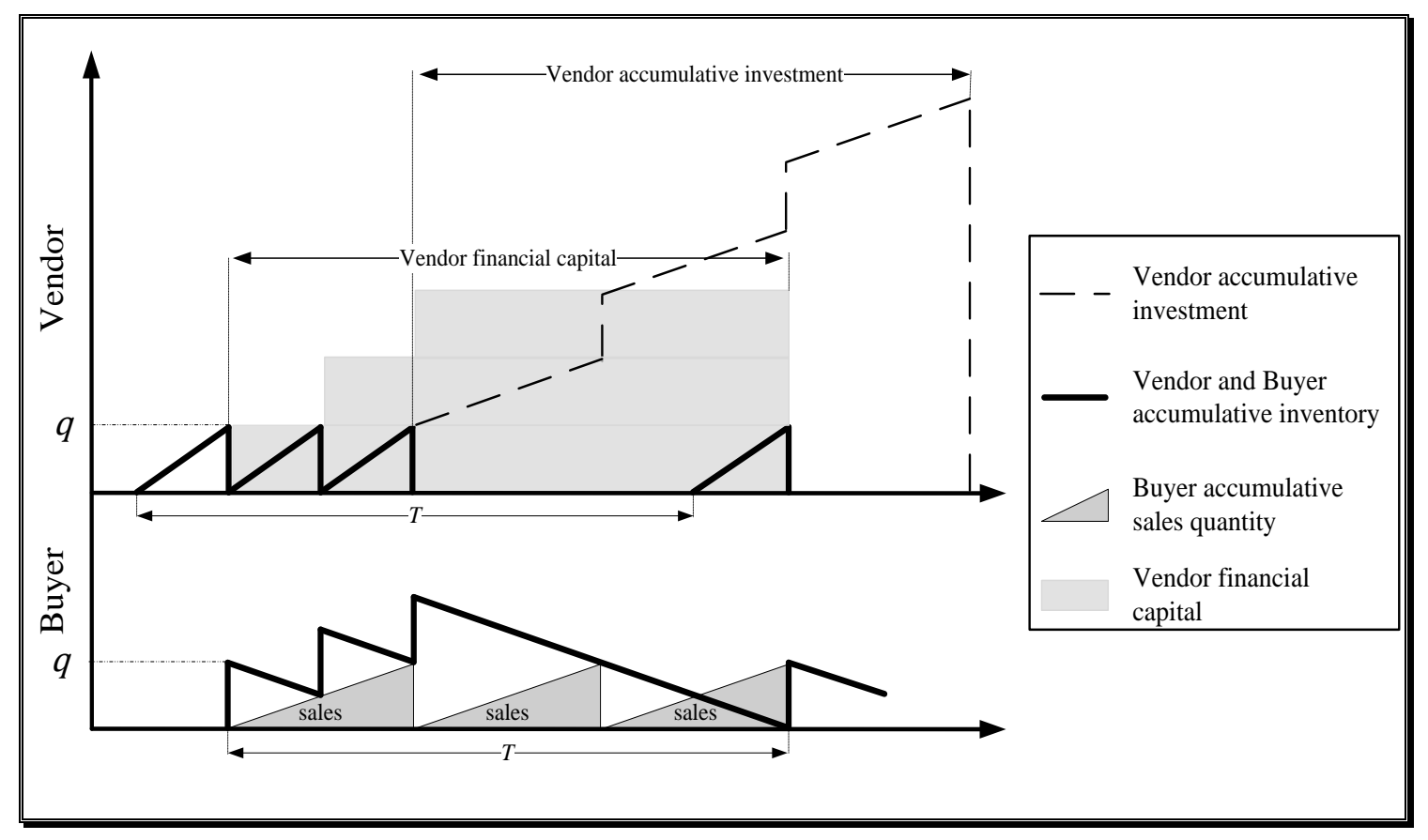

Figure 5-1. The behavior of cash inventory for Scheme 1

The model of Braglia and Zavanella (2003) is modified to include additional costs as per the summary of the contracts in Table 1-2. It is more appropriate here to follow a profit maximization approach. Table 5-1 (next page) provides a comparison between the cost factors that were considered by Braglia and Zavanella (2003) and the ones considered in this model marked in (X).

In addition to the costs considered in Braglia and Zavanella (2003), the vendor incurs additional annual costs, which are the raw material purchasing cost, $c_{p} \gamma D$, the production cost, $c_{p r} D$, the removing and replacing any unsold, unused, or expired product cost, $c_{r} \beta D$, and the audit of inventory cost, $c_{v}^{a} \delta D / n q$. The vendor also incurs the cost of losing the opportunity $\left(C_{v}^{o p}\right)$ to invest the money as it is tied up in the buyer's inventory, which is computed from the sum of the area of 
Table 5-1. Cost comparison with Braglia and Zavanella (2003)

\begin{tabular}{|c|c|c|c|}
\hline \multirow{8}{*}{$\begin{array}{l}\dot{\bar{o}} \\
\frac{0}{0} \\
\end{array}$} & Cost factor & Braglia and Zavanella (2003) & Our model \\
\hline & Setup & $X$ & $\mathrm{X}$ \\
\hline & Holding & $X$ & $X$ \\
\hline & Losing opportunity & & $\mathrm{X}$ \\
\hline & Purchasing & & $X$ \\
\hline & Production & & $\mathrm{X}$ \\
\hline & $\begin{array}{l}\text { Removing and replacing unsold, } \\
\text { unused and expired products }\end{array}$ & & $\mathrm{X}$ \\
\hline & Inventory audit & & $\mathrm{X}$ \\
\hline \multirow{6}{*}{$\underset{\oplus}{\stackrel{\bar{D}}{\vec{D}}}$} & Ordering & $\mathrm{X}$ & $\mathrm{X}$ \\
\hline & Holding & $\mathrm{X}$ & $X$ \\
\hline & Purchasing & & $\mathrm{X}$ \\
\hline & Transaction & & $X$ \\
\hline & Insurance & & $X$ \\
\hline & Inventory audit & & $\mathrm{X}$ \\
\hline
\end{tabular}

the grey rectangles shown in Figure 5-1 at the vendor's side as

$$
C_{v}^{o p}=\frac{c_{b} I_{v} q}{T} \sum_{i=1}^{n}\left(T-(i-1) \frac{q}{P}\right)=c_{b} I_{v} q\left(n-\frac{(n-1) D}{2 P}\right)
$$

where $T=n q / D$, and $c_{b} I_{v}$ is considered as the financial part of the holding cost for the vendor (see i.e. Yi and Sarker, 2013b). Therefore, the vendor's net investment per unit of time $\left(N I_{v}^{1}\right)$ is equal to the investment of each payment (the second part of Eq. 5-5) less the losing opportunity to invest the payment (Eq. 5-1), which is written as

$$
N I_{v}^{1}=c_{b} I_{v} q\left(\frac{(n-1) D}{2 P}-\frac{(m-1) n}{2 m}\right)
$$

Thus, the total cost per unit of time (annual) for the vendor $\left(T C_{v}\right)$ is equal to

$$
T C_{v}=\left(\gamma c_{p}+c_{p r}+\beta c_{r}\right) D+\left(S_{v}+\delta c_{v}^{a}\right) \frac{D}{n q}+h_{v} \frac{q D}{2 P}+c_{b} I_{v} q\left(n-\frac{(n-1) D}{2 P}\right)
$$

The buyer also incurs additional annual costs, which are the purchasing $\operatorname{cost}, c_{b} D$, the insurance $\operatorname{cost}, c_{i} D$, the transaction cost, $c_{t} m D / n q$, and the audit of inventory cost, $c_{b}^{a} \delta D / n q$. The buyer does not incur an opportunity cost from not investing its revenues. Therefore, the total annual (per unit of time) cost for the buyer $\left(T C_{b}\right)$ is equal to 


$$
T C_{b}=\left(c_{b}+c_{i}\right) D+\left(n O_{b}+m c_{t}+\delta c_{b}^{a}\right)\left(\frac{D}{n q}\right)+\frac{h_{b}}{2}\left(n q-(n-1) \frac{q D}{P}\right)
$$

The vendor makes revenue $\left(T R_{v}^{1}\right)$ when the buyer pays for the products sold to its customers (the first part of Eq. 5-5), and from investing each payment (the second part of Eq. 5-5). The vendor's per unit of time (annual) revenue is written as

$$
T R_{v}^{1}=c_{b} D+c_{b} I_{v} q\left(n-\frac{(m-1) n}{2 m}\right)
$$

Similarly, the buyer generates annual revenue $\left(T R_{b}^{1}\right)$ from selling the items to its customers and from investing the amount generated until the due date for each payment (the first and the second part of Eq. 5-6, respectively).

$$
T R_{b}^{1}=c_{c} D+c_{c} I_{b} q\left(\frac{n}{2 m}\right)
$$

Therefore, the total annual (per unit of time) profit $\left(T P_{1}\right)$ of the system is equal to the sum of revenues that the players make minus all their costs, which is written, after some simplification, as

$$
\begin{aligned}
& T P_{1}=\left(c_{c}-\gamma c_{p}-c_{p r}-\beta c_{r}-c_{i}\right) D-\left(S_{v}+\delta c_{v}^{a}+n O_{b}+m c_{t}+\delta c_{b}^{a}\right)\left(\frac{D}{n q}\right)+ \\
& c_{b} I_{v} q\left(\frac{(n-1) D}{2 P}-\frac{(m-1) n}{2 m}\right)+c_{c} I_{b} q\left(\frac{n}{2 m}\right)+\left((n-1) h_{b}-h_{v}\right)\left(\frac{q D}{2 P}\right)-h_{b} \frac{n q}{2}
\end{aligned}
$$

By taking the second derivative of the above equation, it was found that it is negative for all values of $q$ indicating that Eq. (5-7) is concave. The optimal production quantity for Scheme $1\left(q_{1}^{*}\right)$ is found by setting the first derivative for the total system profit $\left(T P_{1}\right)$ equal to zero and solving for $q$. The optimal quantity is found to be equal to

$$
q_{1}^{*}=\sqrt{\frac{O(n, m)}{H_{1}(n, m)}}
$$

where

$O(n, m)=\left(S_{v}+\delta c_{v}^{a}+n O_{b}+m c_{t}+\delta c_{b}^{a}\right)\left(\frac{D}{n}\right)$ 
and

$H_{1}(n, m)=\frac{h_{b} n}{2}-c_{b} I_{v}\left(\frac{(n-1) D}{2 P}-\frac{(m-1) n}{2 m}\right)-c_{c} I_{b}\left(\frac{n}{2 m}\right)-\frac{\left((n-1) h_{b}-h_{v}\right) D}{2 P}$

\subsection{Scheme 2: Payments upon receiving shipments}

Different from the previous scheme, this scheme assumes that the buyer pays the vendor when it receives a shipment for the quantity that has been sold since the last payment. The buyer pays the vendor $(n-1)$ payments that are equal to $c_{b} D(q / P)$ each at time $q / P, 2 q / P, \ldots,(n-1) q / P$, and a single payment that is equal to $c_{b} D(T-(n-1) q / P)$ at time $T$, where $m=n$. The behavior of the cash inventory for the vendor and the buyer for Scheme 2 is shown in Figure 5-2.

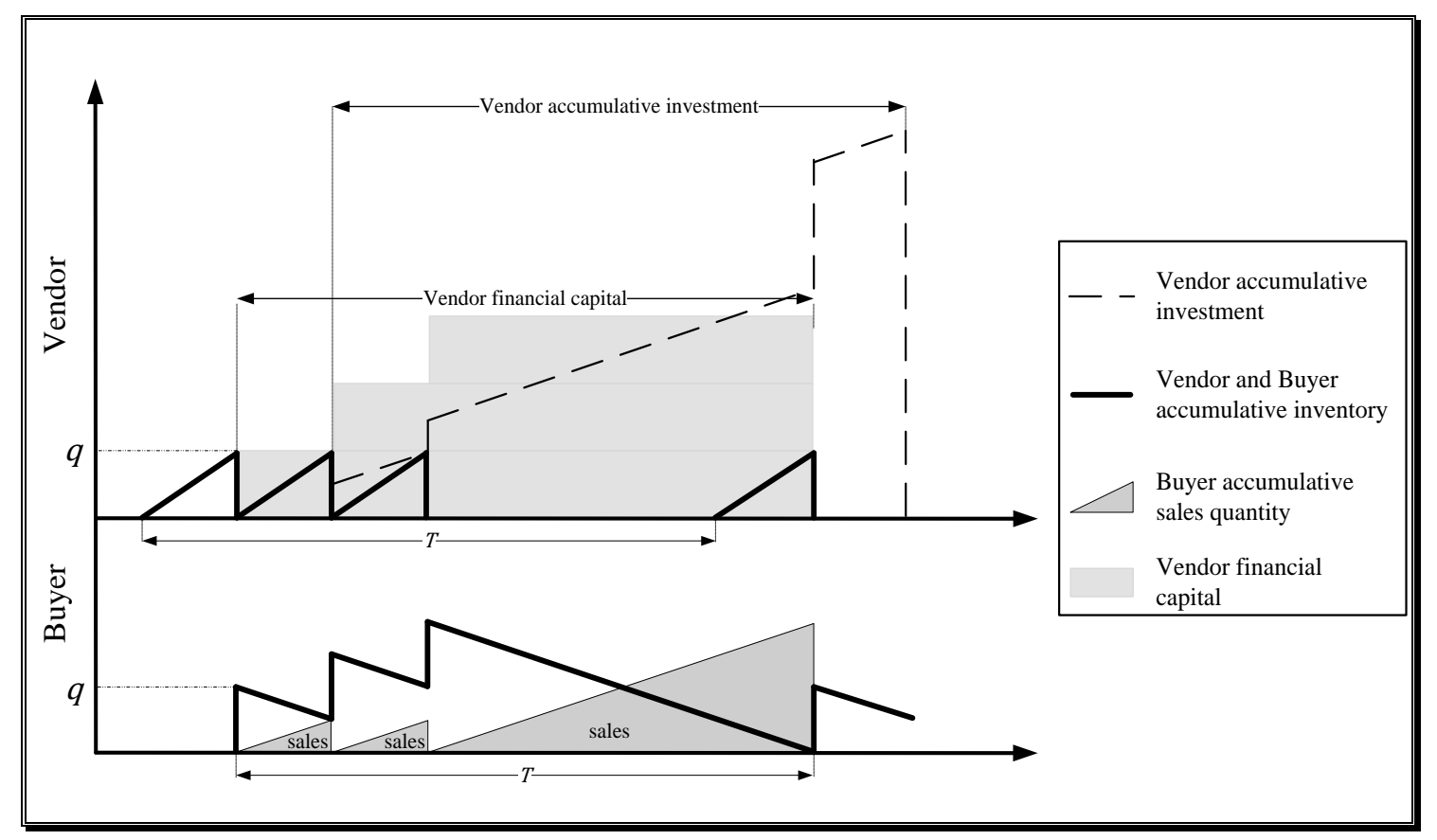

Figure 5-2. The behavior of cash inventory for Scheme 2

Both players incur the same costs as those described in Scheme 1. The vendor's net investment per unit of time $\left(N I_{v}^{2}\right)$ according to Figure 5-2 is rewritten as

$$
N I_{v}^{2}=c_{b} I_{v} q\left(\frac{2 n(D-P)+(n-1) D}{2 P}-\frac{(n-1) D^{2}((n-2) D-2)}{2 n P^{2}}\right)
$$


The vendor's and the buyer's revenues per unit of time, $\left(T R_{v}^{2}\right)$ and $\left(T R_{b}^{2}\right)$, respectively, are modified in accordance with Figure 5-2 as:

$$
\begin{aligned}
& T R_{v}^{2}=c_{b} D+c_{b} I_{v} q\left(\frac{n D}{P}-\frac{(n-1) D^{2}((n-2) D-2)}{2 n P^{2}}\right) \\
& T R_{b}^{2}=c_{c} D+c_{c} I_{b} q\left(\frac{n(P-D)^{2}-(D-2 P) D}{2 P^{2}}\right)
\end{aligned}
$$

The total annual profit $\left(T P_{2}\right)$ of the system is written similar to Eq. (5-7) as

$$
\begin{aligned}
& T P_{2}=\left(c_{c}-\gamma c_{p}-c_{p r}-\beta c_{r}-c_{i}\right) D-\left(S_{v}+\delta c_{v}^{a}+n O_{b}+m c_{t}+\delta c_{b}^{a}\right)\left(\frac{D}{n q}\right)+ \\
& c_{b} I_{v} q\left(\frac{2 n(D-P)+(n-1) D}{2 P}-\frac{(n-1) D^{2}((n-2) D-2)}{2 n P^{2}}\right)+c_{c} I_{b} q\left(\frac{n(P-D)^{2}-(D-2 P) D}{2 P^{2}}\right)+((n- \\
& \text { 1) } \left.h_{b}-h_{v}\right)\left(\frac{q D}{2 P}\right)-h_{b}\left(\frac{n q}{2}\right)
\end{aligned}
$$

Eq. (5-12) is also concave, and the optimum quantity for Scheme $2\left(q_{2}^{*}\right)$ that maximizes Eq. (5-12) is given as

$$
q_{2}^{*}=\sqrt{\frac{O(n, m)}{H_{2}(n)}}
$$

where

$$
\begin{gathered}
H_{2}(n)=\frac{n h_{b}}{2}-c_{b} I_{v}\left(\frac{2 n(D-P)+(n-1) D}{2 P}-\frac{(n-1) D^{2}((n-2) D-2)}{2 n P^{2}}\right) \\
-c_{c} I_{b}\left(\frac{n(P-D)^{2}-(D-2 P) D}{2 P^{2}}\right)-\frac{\left((n-1) h_{b}-h_{v}\right) D}{2 P}
\end{gathered}
$$

\subsection{Scheme 3: Two unequal payments with largest at the end}

Unlike the previous models, this model assumes that the buyer makes two payments $m=2$. The first one is due when the buyer receives the last shipment $(n-1) q / P$ while the second payment is due by the end of the cycle. The behavior of the cash inventory for the vendor and the buyer for Scheme 3 is shown in Figure 5-3 (next page). 


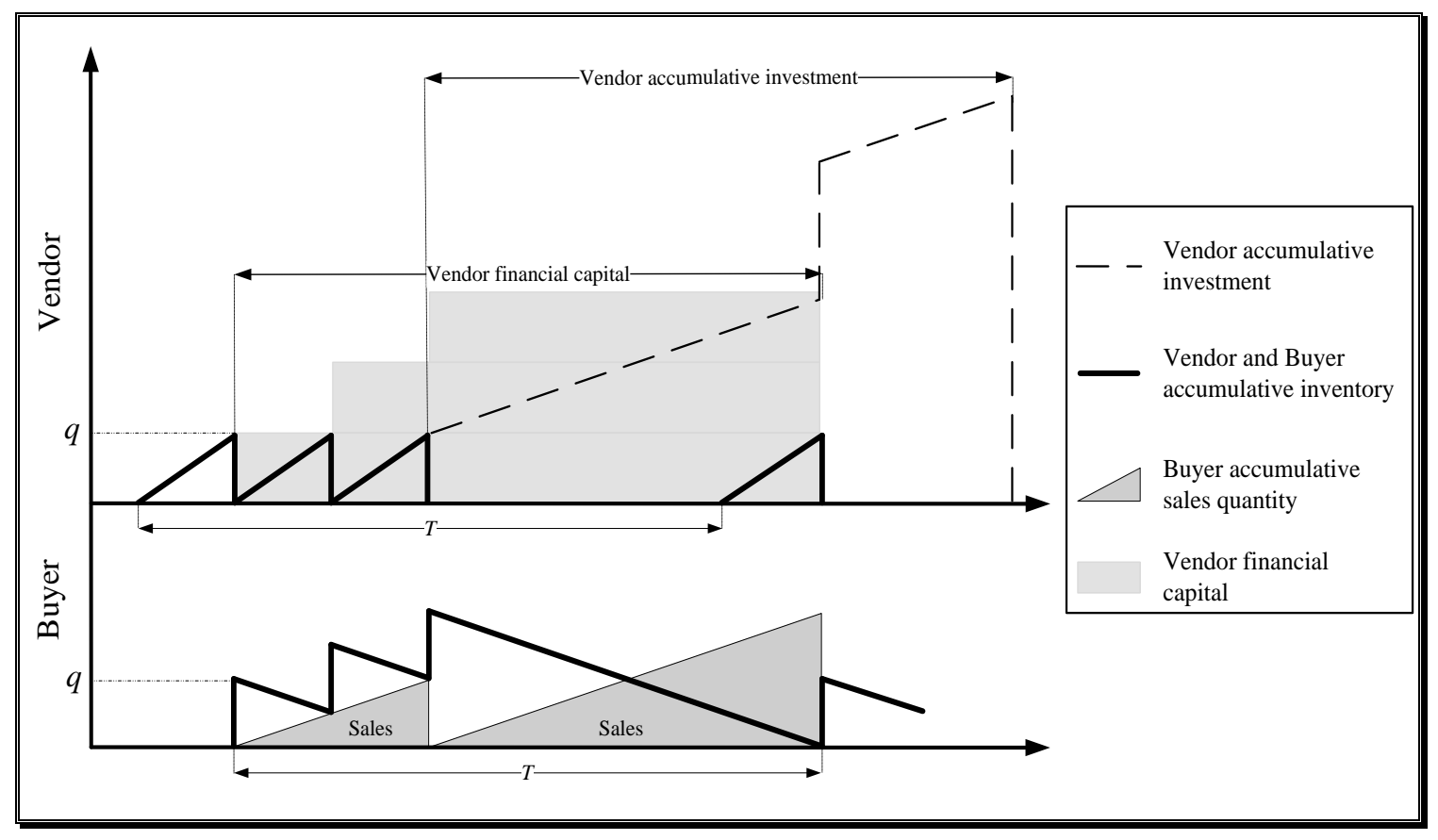

Figure 5-3. The behavior of cash inventory for Scheme 3

Both players incur the same costs as those described in Scheme 1. The vendor's net investment per unit of time $\left(N I_{v}^{3}\right)$ according to Figure 5-3 is rewritten as

$$
N I_{v}^{3}=c_{b} I_{v} q\left(\frac{2(n-1) D+(n-1) D / 2}{P}-\frac{n\left(P^{2}+D^{2}\right)+(2 n-1) D^{2} / n}{P^{2}}\right)
$$

Eqs. (5-5 and 5-6) are modified in accordance with Figure 5-3 to find the vendor's $\left(T R_{v}^{3}\right)$ and the buyer's $\left(T R_{b}^{3}\right)$ revenues per unit of time as:

$$
\begin{aligned}
& T R_{v}^{3}=c_{b} D+c_{b} I_{v} q\left(\frac{(2 P-D) n D / P+(2 n-1) D^{2} / n P-2 D}{P}\right) \\
& T R_{b}^{3}=c_{c} D+c_{c} I_{b} q\left(\frac{(n-1)^{2} D^{2} / n P+n(P-2 D) / 2+D}{P}\right)
\end{aligned}
$$

The total annual profit $\left(\mathrm{TP}_{3}\right)$ of the system is written similar to Eq. (5-7) as 


$$
\begin{aligned}
T P_{3}= & \left(c_{c}-\gamma c_{p}-c_{p r}-\beta c_{r}-c_{i}\right) D-\left(S_{v}+\delta c_{v}^{a}+n O_{b}+m c_{t}+\delta c_{b}^{a}\right)\left(\frac{D}{n q}\right)+ \\
& c_{b} I_{v} q\left(\frac{2(n-1) D+(n-1) D / 2}{P}-\frac{n\left(P^{2}+D^{2}\right)+(2 n-1) D^{2} / n}{P^{2}}\right)+ \\
& c_{c} I_{b} q\left(\frac{(n-1)^{2} D^{2} / n P+n(P-2 D) / 2+D}{P}\right)+\left((n-1) h_{b}-h_{v}\right)\left(\frac{q D}{2 P}\right)-h_{b} \frac{n q}{2}
\end{aligned}
$$

Eq. (5-17) is also concave, and the optimum quantity for Scheme $3\left(q_{3}^{*}\right)$ that maximizes Eq. (5-17) is given as

$$
q_{3}^{*}=\sqrt{\frac{O(n, m)}{H_{3}(n)}}
$$

where

$$
\begin{aligned}
H_{3}(n)=\frac{h_{b} n}{2} & -c_{b} I_{v}\left(\frac{2(n-1) D+(n-1) D / 2}{P}-\frac{n\left(P^{2}+D^{2}\right)+(2 n-1) D^{2} / n}{P^{2}}\right) \\
& -c_{c} I_{b}\left(\frac{(n-1)^{2} D^{2} / n P+n(P-2 D) / 2+D}{P}\right)-\left((n-1) h_{b}-h_{v}\right)\left(\frac{D}{2 P}\right)
\end{aligned}
$$

\subsection{Scheme 4: Two unequal payments with smallest at the end}

In this payment scheme, one can realize that the due date for the first payment is $(T-q / P)$ where the second payment is made at the end of the cycle, which means $m=2$. The reason behind considering this scheme is to test the effect of delaying the first payment as much as the vendor can, which is opposite of the third scheme. The behavior of the cash inventory for the vendor and the buyer for Scheme 4 is shown in Figure 5-4 (next page).

Both players incur the same costs as those described in Scheme 1. The vendor's net investment per unit of time $\left(N I_{v}^{4}\right)$ according to Figure 5-4 is rewritten as

$$
N I_{v}^{4}=c_{b} I_{v} q\left(\frac{(n-1) D}{2 P}-\frac{D^{2}}{n P^{2}}\right)
$$

Eqs. (5-5) and (5-6) are modified in accordance with Figure 5-4 to find the vendor's $\left(T R_{v}^{4}\right)$ and the buyer's $\left(T R_{b}^{4}\right)$ revenues per unit of time which are written as: 


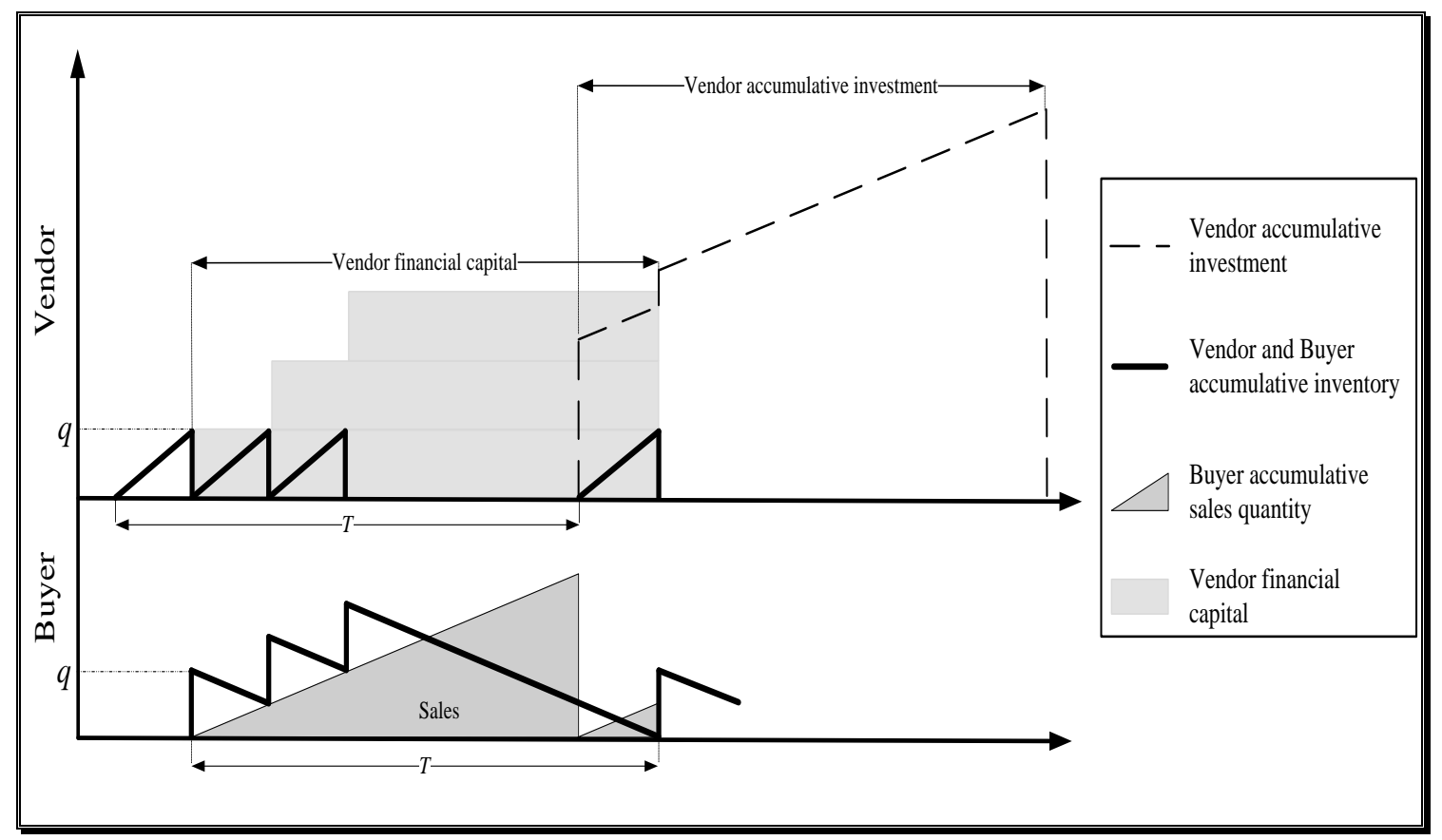

Figure 5-4. The behavior of cash inventory for Scheme 4

$$
\begin{aligned}
& T R_{v}^{4}=c_{b} D+c_{b} I_{v} q\left(n-\frac{D^{2}}{n P^{2}}\right) \\
& T R_{b}^{4}=c_{c} D+c_{c} I_{b} q\left(\frac{n P-2 D}{2 P}+\frac{D^{2}}{n P^{2}}\right)
\end{aligned}
$$

The total annual profit $\left(\mathrm{TP}_{4}\right)$ of the system is written similar to Eq. $(5-7)$ as

$$
\begin{aligned}
& T P_{4}=\left(c_{c}-\gamma c_{p}-c_{p r}-\beta c_{r}-c_{i}\right) D-\left(S_{v}+n O_{b}+\delta c_{v}^{a}+m c_{t}+\delta c_{b}^{a}\right)\left(\frac{D}{n q}\right)+ \\
& c_{b} I_{v} q\left(\frac{(n-1) D}{2 P}-\frac{D^{2}}{n P^{2}}\right)+c_{c} I_{b} q\left(\frac{n P-2 D}{2 P}+\frac{D^{2}}{n P^{2}}\right)+\left((n-1) h_{b}-h_{v}\right)\left(\frac{q D}{2 P}\right)-h_{b} \frac{n q}{2}
\end{aligned}
$$

Eq. (5-22) is also concave, and the optimum quantity for Scheme $4\left(q_{4}^{*}\right)$ that maximizes Eq. (5-22) is given as

$$
q_{4}^{*}=\sqrt{\frac{O(n, m)}{H_{4}(n)}}
$$

where 
$H_{4}(n)=h_{b} \frac{n}{2}-c_{b} I_{v}\left(\frac{(n-1) D}{2 P}-\frac{D^{2}}{n P^{2}}\right)-c_{c} I_{b}\left(\frac{n P-2 D}{2 P}+\frac{D^{2}}{n P^{2}}\right)-\left((n-1) h_{b}-h_{v}\right)\left(\frac{D}{2 P}\right)$

\subsection{Solution Procedure}

This section illustrates the procedure used to find the optimal solution (number of shipments $\left(n^{*}\right)$, payments $\left(m^{*}\right)$, and quantity $\left.\left(q_{i}^{*}\right)\right)$ that maximizes the total profit of the system for each payment scheme described in the previous sections. The solution procedure was programmed in Microsoft Excel, using Visual Basic for Applications (VBA) with nested search loops. The procedure is very similar to the one described in Jaber and Goyal (2008).

1. Input the parameters for the buyer $\left(D, h_{b}, \delta, I_{b}, c_{b}, c_{i}, c_{c}, O_{b}, c_{t}, c_{b}^{a}\right)$, and the vendor $\left(P, h_{v}, \gamma, \beta, \delta, I_{v}, c_{p}, c_{p r}, c_{r}, c_{v}^{a}, S_{v}\right)$

2. Set $\left(n_{j}, m_{y}\right)$ to be the number of shipments $\left(n_{j}=j\right)$ and the number of payments $\left(m_{y}=y\right)$, respectively, where $j$ and $y=1,2, \ldots$ etc.

3. Find the optimum quantity of the system $\left(q_{i}^{*}\right)$ for Scheme $i$, where $i=1,2,3$ and 4 , using the values in Steps 1 and 2, and Eqs. (5-8), (5-13), (5-18), and (5-23) for Schemes 1, 2, 3 and 4 , respectively.

4. Calculate the total profit of the system $T P_{i}$ for $i=1,2,3$ and 4 , using the values in Steps 1, 2 and 3, and Eqs. (5-7), (5-12), (5-17) and (5-22) for Schemes 1, 2, 3 and 4, respectively.

5. Repeat Steps 3 and 4 for $\left(n_{j}, m_{y}+1\right)$ and compare it with the system total profit for $\left(n_{j}, m_{y}\right)$, e.g. when $j=1, y=1, n_{1}=1$, and $m_{1}=1$. If the system total profit is such that $T P_{i}\left(n_{j}, m_{y}+1\right)>T P_{i}\left(n_{j}, m_{y}\right)$, then set $\left(n_{j}, m_{y}+1\right)$ to be your new $\left(n_{j}, m_{y}\right)$, and repeat Steps 3 and 4 for $\left(n_{j}, m_{y}+2\right)$. Continue until $T P_{i}$ for $T P_{i}\left(n_{j}, m_{y}\right)>T P_{i}\left(n_{j}, m_{y}-\right.$ $1)$ and $T P_{i}\left(n_{j}, m_{y}\right)>T P_{i}\left(n_{j}, m_{y}+1\right)$. Then, stop and go for the next step. Set $\left(n_{j}, m_{y}\right)$ that give the highest $T P_{i}$ to be the optimal solution for this iteration with the optimum number of shipments and payments $\left(n_{j}^{*}, m_{y}^{*}\right)$ and the optimum quantity $\left(q_{i}^{*}\right)$.

6. Repeat Steps 3 and 4 for $\left(n_{j}+1, m_{y}\right)$ and compare the system's total profit with the one from Step 5 for $\left(n_{j}^{*}, m_{y}^{*}\right)$. If $T P_{i}\left(n_{j}+1, m_{y}\right)>T P_{i}\left(n_{j}^{*}, m_{y}^{*}\right)$, then set $\left(n_{j}+1, m_{y}\right)$ to be 
the new $\left(n_{j}, m_{y}\right)$ values, and repeat Steps 3 and 4 for $\left(n_{j}+1, m_{y}+1\right)$. Continue until $T P_{i}\left(n_{j}, m_{y}\right)>T P_{i}\left(n_{j}, m_{y}-1\right)$ and $T P_{i}\left(n_{j}, m_{y}\right)>T P_{i}\left(n_{j}, m_{y}+1\right)$. Then, stop and set $\left(n_{j}, m_{y}\right)$ that give the highest $T P_{i}$ to be the optimal solution for that payment scheme with the optimum number of shipments and payments $\left(n_{j}^{*}, m_{y}^{*}\right)$ and optimum quantity $\left(q_{i}^{*}\right)$ to replace those in Step 5.

7. Now, after finding $\left(q_{i}^{*}, n_{j}^{*}, m_{y}^{*}\right)$, use these values to calculate the vendor's and buyer's total $\operatorname{cost}\left(T C_{v}\right)$ and $\left(T C_{b}\right)$ using Eqs. (5-3) and (5-4), respectively.

8. To find the total revenue for each player for Scheme 1, 2, 3 and 4, Eqs. (5-5), (5-10), (5-15) and (5-20) are used for the vendor $\left(T R_{v}\right)$, respectively, where Eqs. (5-6), (5-11), (5-16) and $(5-21)$, respectively, are used for the buyer $\left(T R_{b}\right)$ using $\left(q_{i}^{*}, n_{j}^{*}, m_{y}^{*}\right)$.

9. Finally, to calculate the total profit for each player, $\left(T P_{v}\right)$ and $\left(T P_{b}\right)$, subtract the total cost for the player, found in Step 7, from its total revenue, found in Step 8.

Note that, in Scheme 2, $n_{j}=m_{y}$ where, in Schemes 3 and 4, $m_{y}=2$.

\subsection{Numerical Example}

In this section, a numerical example is solved to illustrate the behavior of the developed models and to compare the results of the four payment schemes. To solve the numerical example, some of the values were adopted from Braglia and Zavanella (2003) while the others were logically (from published studies) estimated to meet the assumptions that were considered in this chapter. For example, $c_{b}=\left(c_{p}+c_{p r}\right) 1.35, c_{i}=0.013 c_{b}, c_{c}=1.35 c_{b}$. Table 5-2 below represents the numbers that were used in this example.

Table 5-2. Values used to solve the numerical example

\begin{tabular}{cccccccccccccccccccc}
\hline$P$ & $D$ & $h_{v}$ & $h_{b}$ & $\gamma$ & $\beta$ & $\delta$ & $I_{v}$ & $I_{b}$ & $c_{p}$ & $c_{p r}$ & $c_{r}$ & $c_{b}$ & $c_{i}$ & $c_{c}$ & $c_{v}^{a}$ & $S_{v}$ & $O_{b}$ & $c_{t}$ & $c_{b}^{a}$ \\
3200 & 1000 & 4 & 5 & 1 & 0.02 & 2 & 0.1 & 0.2 & 3 & 1 & 10 & 5.4 & 0.07 & 7.29 & 4 & 400 & 25 & 0.5 & 5 \\
\hline
\end{tabular}


The steps of the solution procedure described in Section 5.5 were used to find the optimal solution for each payment scheme as seen in Table 5-3.

Table 5-3. Results for the optimal solution for the numerical example

\begin{tabular}{ccccccccccc}
\hline Scheme & $n$ & $m$ & $q$ & $T C_{v}(\$)$ & $T R_{v}(\$)$ & $T P_{v}(\$)$ & $T C_{b}(\$)$ & $T R_{b}(\$)$ & $T P_{b}(\$)$ & $T P_{S}(\$)$ \\
\hline 1 & 5 & 1 & 134 & $5,208.7$ & $5,762.9$ & 554.2 & $6,931.7$ & $7,779.9$ & 848.2 & $\mathbf{1 , 4 0 2 . 5}$ \\
2 & 2 & 2 & 231 & $5,457.9$ & $5,484.0$ & 26.1 & $6,575.9$ & $7,537.8$ & $\mathbf{9 6 1 . 9}$ & 988.0 \\
3 & 4 & 2 & 113 & $5,387.2$ & $5,501.3$ & 114.1 & $6,582.3$ & $7,501.8$ & 919.5 & $1,033.6$ \\
4 & 6 & 2 & 113 & $5,189.9$ & $5,766.7$ & $\mathbf{5 7 6 . 8}$ & $6,965.4$ & $7,737.4$ & 771.9 & $1,348.7$ \\
\hline
\end{tabular}

The results in Table 5-3 show that the best payment scheme in which the system makes more profit is when the buyer pays the vendor frequent equal payments, which is in this example equal to one payment $(m=1)$ at the end of the cycle. The best payment scheme for the buyer is Scheme 2: Payments upon receiving shipments in which it makes $\$ 961.9$ as a profit even though the system makes the least profit using this payment scheme. On the other hand, the vendor makes the highest profit using Scheme 4. The second profitable payment scheme for the whole system is Scheme 4: Two unequal payments with the smallest at the end. The results in Table 5-3 also show that it is more profitable for the system when the payment is close to the end of the cycle. The reason behind this is that the system's revenue from investing the money is higher as the buyer has longer time to invest the money (generated from sales) before paying the vendor. This helps the buyer to cover its additional expenses such as increasing holding, buying insurance premium, and inventory audit costs, which occur from adopting the CS agreement (see Table 5-4).

Table 5-4. Breakdown of all variable costs and revenue in dollars for the vendor, the buyer, and the system

\begin{tabular}{|c|c|c|c|c|c|c|c|c|c|c|c|c|}
\hline \multirow[b]{2}{*}{ Cost } & \multicolumn{3}{|c|}{ Scheme 1} & \multicolumn{3}{|c|}{ Scheme 2} & \multicolumn{3}{|c|}{ Scheme 3} & \multicolumn{3}{|c|}{ Scheme 4} \\
\hline & $\mathrm{V}$ & $\mathrm{B}$ & $S$ & $\mathrm{~V}$ & $\mathrm{~B}$ & $S$ & $\mathrm{~V}$ & B & $S$ & $\mathrm{~V}$ & $\mathrm{~B}$ & $S$ \\
\hline Set-up & 595.2 & & 595.2 & 866.5 & & 866.5 & 882.7 & & 882.7 & 587.5 & & 587.5 \\
\hline Holding & 84.0 & $1,260.0$ & $1,344.0$ & 144.3 & 973.7 & $1,118.0$ & 70.8 & 867.4 & 938.2 & 70.9 & $1,259.0$ & $1,329.9$ \\
\hline $\begin{array}{l}\text { Losing } \\
\text { Opportunity }\end{array}$ & 317.5 & & 317.5 & 229.8 & & 229.8 & 216.0 & & 216.0 & 319.8 & & 319.8 \\
\hline $\begin{array}{l}\text { Inventory } \\
\text { audit }\end{array}$ & 11.9 & 14.9 & 26.8 & 17.3 & 21.7 & 39.0 & 17.7 & 22.1 & 39.7 & 11.7 & 14.7 & 26.4 \\
\hline Ordering & & 186.0 & 186.0 & & 108.3 & 108.3 & & 220.7 & 220.7 & & 220.3 & 220.3 \\
\hline Transaction & & 0.7 & 0.7 & & 2.2 & 2.2 & & 2.2 & 2.2 & & 1.5 & 1.5 \\
\hline Insurance & & 70.0 & 70.0 & & 70.0 & 70.0 & & 70.0 & 70.0 & & 70.0 & 70.0 \\
\hline \multicolumn{13}{|l|}{ Revenue } \\
\hline Investment & 362.9 & 489.9 & 852.8 & 84.0 & 247.8 & 331.8 & 101.3 & 211.8 & 313.1 & 366.7 & 447.4 & 814.1 \\
\hline
\end{tabular}

(V: vendor, B: buyer and S: system) 
In addition to the numerical example above, 2,000 numerical examples were generated and solved using nested loop search written using Visual Basic for Applications (VBA) codes, similar to the one provided in Jaber and Goyal (2008). The values for each parameter were randomly generated, and the optimum total profit for the system $\left(T P_{S}\right)$ was found for each payment scheme. Some conditions were applied when generating these examples to meet the assumptions such as the demand is less than the production rates. Table 5-5 (next page) shows the minimum (Min) and the maximum (Max) ranges, and the average (Avg) and the standard deviation (SD) of the generated values for the parameters of the 2,000 examples. After finding the optimum solutions for the 2,000 numerical examples, which were solved for each payment scheme, a comparison among the results was performed to determine the ranking of the payment schemes. The results and the analysis for these examples showed that Scheme 1 performed the best by registering the highest total profit in $100 \%$ of the 2,000 numerical examples. Scheme 2 returned the second highest total profit in $13.35 \%$ of the 2,000 examples, the third highest total profit in $78.45 \%$ of the examples, and was the lowest in $8.2 \%$ of the 2,000 examples. For Scheme 3, the analysis showed that it returned the third highest total profit in $17.3 \%$ of the 2,000 examples, and was the lowest in $82.7 \%$ of the 2,000 examples. Moreover, the analysis showed that Scheme 4 returned the second highest total profit in $86.65 \%$ of the examples, the third highest total profit in $4.25 \%$ of the total number of examples, and was the lowest in $9.1 \%$ of the 2,000 examples. Therefore, the payment schemes are ranked from 1 to 4 based on the highest percentage that was achieved by each one of them. In this case, Scheme 1 was the best, followed by Scheme 4, Scheme 2, and Scheme 3, respectively.

As this chapter is an extension of the work of Braglia and Zavanella (2003), it is better to compare the results above with their work to show the effects that the additional costs and revenues have on the total profit of the system. The total profit per unit of time for the model of Braglia and Zavanella (2003) is equal to the total revenue for the system less the total costs (Eq. 4-6), which is written as

$P_{S}=\left(c_{c}-\gamma c_{p}\right) D-\left(A_{v}+n A_{b}\right) \frac{D}{n q}-h_{2}\left(\frac{q D}{P}+n q \frac{P-D}{2 P}\right)+\left(h_{2}-h_{1}\right) \frac{q D}{2 P}$ 
Table 5-5. Minimum and maximum ranges, and average and standard deviation values for each parameter for 2,000 numerical examples

\begin{tabular}{ccccc}
\cline { 2 - 4 } Parameter & \multicolumn{2}{c}{ Range } & $\begin{array}{c}\text { Average of generated } \\
\text { examples }\end{array}$ & $\begin{array}{c}\text { Standard deviation of } \\
\text { generated examples }\end{array}$ \\
\cline { 2 - 4 }$P$ & $1,000.00$ & $11,000.00$ & $8,261.80$ & $2,012.60$ \\
$D$ & $1,000.00$ & $6,000.00$ & $1,663.40$ & 779.40 \\
$h_{v}$ & 1.00 & 4.00 & 2.60 & 1.10 \\
$h_{b}$ & 3.00 & 8.00 & 6.00 & 1.60 \\
$\gamma$ & 1.00 & 1.00 & 1.00 & 0.00 \\
$\beta$ & 0.00 & 0.05 & 0.03 & 0.01 \\
$\delta$ & 1.00 & 3.00 & 2.00 & 0.80 \\
$I_{v}$ & 0.00 & 0.40 & 0.12 & 0.11 \\
$I_{b}$ & 0.00 & 0.40 & 0.18 & 0.11 \\
$c_{p}$ & 1.00 & 4.00 & 2.20 & 1.10 \\
$c_{p r}$ & 1.00 & 3.00 & 1.80 & 0.80 \\
$c_{r}$ & 1.00 & 10.00 & 5.60 & 2.90 \\
$c_{b}$ & 2.70 & 9.50 & 5.50 & 1.90 \\
$c_{i}$ & 0.02 & 0.07 & 0.04 & 0.01 \\
$c_{c}$ & 3.60 & 12.80 & 7.40 & 2.50 \\
$c_{v}^{a}$ & 1.00 & 5.00 & 3.00 & 1.40 \\
$S_{v}$ & 100.00 & 800.00 & 450.10 & 199.50 \\
$O_{b}$ & 10.00 & 200.00 & 109.60 & 58.00 \\
$c_{t}$ & 0.50 & 1.00 & 0.80 & 0.10 \\
$c_{b}^{a}$ & 2.00 & 7.00 & 4.50 & 1.70 \\
\hline
\end{tabular}

The terms $\left(c_{c}-\gamma c_{p}\right) D$ are the buyer's selling price and the vendor's raw material purchasing cost per unit of time, respectively. The total cost, without the added terms, in Braglia and Zavanella (2003) was $\$ 2,035$ with $q^{*}=123$, and $n^{*}=4$. Therefore, the annual total profit for their model in dollars is $P_{S}=(7.29-1(3)) 1,000-2,035=\$ 2,255$ without any changes in $q^{*}$ or $n^{*}$. On the other hand, if the transaction cost per unit of time, $c_{t} m D / n q$, is added to their model, which implied that the vendor is paid on a daily basis, the above equation is rewritten as

$P_{S}=\left(c_{c}-\gamma c_{p}\right) D-\left(A_{v}+n A_{b}+365 c_{t}\right) \frac{D}{n q}-h_{2}\left(\frac{q D}{P}+n q \frac{P-D}{2 P}\right)+\left(h_{2}-h_{1}\right) \frac{q D}{2 P}$

With

$$
q^{*}=\sqrt{\frac{\frac{\left(A_{v}+n A_{b}+365 c_{t}\right) D}{n}}{h_{2}\left(\frac{D}{P}+\frac{n(P-D)}{2 P}\right)-\frac{\left(h_{2}-h_{1}\right) D}{2 P}}}
$$


As a result, the total profit for their system per unit of time after the modifications is $P_{S}=\$ 1,913$ with $q^{*}=144$, and $n^{*}=4$. Since Braglia and Zavanella (2003) considered equal frequent payments in their model, Scheme 1 (with all costs and revenue considered in this chapter) was solved according to what was reported by them that the vendor is paid daily $(m=365)$, and the results are shown in Table 5-6.

Table 5-6. Optimal results for the total profit for B\&Z, Scheme $1+B \& Z$, and Scheme 1, 2, 3 and 4

\begin{tabular}{ccccccc} 
& B\&Z & Scheme 1+B\&Z & Scheme 1 & Scheme 2 & Scheme 3 & Scheme 4 \\
\hline Total Profit (\$) & 1,913 & 495.6 & $1,402.5$ & 988.0 & $1,033.6$ & $1,348.7$ \\
$q^{*}$ & 144 & 139 & 134 & 231 & 113 & 113 \\
$n^{*}$ & 4 & 4 & 5 & 2 & 4 & 6 \\
$m^{*}$ & 365 & 365 & 1 & 2 & 2 & 2 \\
\hline
\end{tabular}

(B\&Z: Braglia and Zavanella (2003))

The B\&Z column represents the optimal results and the total profit for the model of Braglia and Zavanella (2003) where the buyer pays the vendor for each item withdrawn, say daily $(m=365)$. It does not consider investing the sales revenue or any additional cost factors (i.e. removing and replacing unused, unsold or expired products, insurance, and inventory audit). The B\&Z model is simple and does not reflect real CS contracts. The second column, Scheme $1+\mathrm{B} \& \mathrm{Z}$, solves the example in column 3 assuming 365 payments. The results show that the profit reduces significantly because of transaction costs and that the model of $\mathrm{B} \& \mathrm{Z}$ overestimates the system profit. The remaining four columns are taken from Table 5-3. Although B\&Z gives the higher total profit, the results do not reflect the reality of the CS agreement. If the revenues and the cost factors, which were adopted from the CS contracts, are considered, paying the vendor on a daily basis gives the least total profit (Scheme 1+B\&Z). The results in Table 5-6 show that finding the optimal number of transactions provides higher system profit than paying the vendor on a daily basis.

As seen in the numerical example (Table 5-3), some payment schemes return better results and higher profits while the other schemes return lower profits. Although the system makes more profit using one payment scheme than the other schemes, the results showed that the difference in profits for the players is very large. The results in Table 5-7 (next page) show the standard deviation of the profits between the vendor and the buyer. 
Table 5-7. Deviation between the vendor's and the buyer's total profit

\begin{tabular}{cccc}
\hline Scheme & $T P_{v}(\$)$ & $T P_{b}(\$)$ & $\sigma$ \\
\hline 1 & 554.2 & 848.2 & 207.9 \\
2 & 26.1 & 961.9 & 661.7 \\
3 & 114.1 & 919.5 & 569.5 \\
4 & 576.8 & 771.9 & 138.0 \\
\hline
\end{tabular}

(TP: total profit; subscripts $v=$ vendor, $b=$ buyer)

Accordingly, incorporating a profit sharing mechanism with the CS agreement is helpful for the players as it may reduce the difference in profits and enhance the results. Also, the profit sharing, as an incentive, plays a major role in the player's decision because the buyer does not accept to adopt a consignment policy unless it provides the same, or better, profit. The next section investigates the benefits of incorporating a profit sharing mechanism with the CS agreement.

\subsection{Profit Sharing Scenarios}

There are different profit sharing mechanisms in the literature (e.g., Jaber and Osman, 2006; Jaber et al. 2006). These mechanisms usually are adopted to compensate the party that loses money as a result of accepting a new coordination mechanism. Also, they are used to reduce the variance between the profits of the vendor and the buyer. In addition, these mechanisms are used to convince the player to accept a new mechanism and change its decision. As a result, three profit sharing scenarios, as examples, are investigated in this section and the results are compared to the numerical example in Section 5.6. In this research, it is assumed that the players do not adopt any coordination mechanism that lowers their profits.

\subsubsection{Scenario 1: Equal return on investment}

This scenario suggests that each player gets a portion from the total profit of the system proportional to its invested money (Equal Return on Investment (ROI)). To illustrate, if the buyer's total cost after the coordination is $\left(T C_{b}\right)$ and the vendor's total cost is $\left(T C_{v}\right)$, the buyer's proportion from the total system profit $\left(T P_{s}\right)$ after the coordination is equal to $\left(T P_{s} \cdot T C_{b} / T C_{s}\right)$ and the vendor's profit is equal to $\left(T P_{S} \cdot T C_{v} / T C_{s}\right)$ where $\left(T C_{S}\right)$ is the total cost of the system. Therefore, the player that gets more profit than what it should, using this mechanism, compensates the other player and pay the difference. Using this profit sharing mechanism guarantees that all players get the same return on investment (ROI). 
After applying this profit sharing mechanism to the numerical example above, the results were found as shown in Table 5-8. The vendor's total cost $\left(T C_{v}\right)$ and the buyer's total cost $\left(T C_{b}\right)$ are obtained from Table 5-3. The system's total $\operatorname{cost}\left(T C_{S}\right)$ is the summation of the total costs for both players. Also, as mentioned earlier, the system's total profit $\left(T P_{S}\right)$ for each scheme is calculated using Eqs. (5-7), (5-12), (5-17), and (5-22). To calculate how much the adjusted profit is for each player, the percentage of the investment for each player from the total investment of the system $\left(T C_{v} / T C_{s}\right.$ and $\left.T C_{b} / T C_{s}\right)$ is found, and then multiplied by the total profit of the system $\left(T P_{s}\right)$. To find the ROI of each player, the total profit for each player after the adjustment is divided by the total cost of the player. The results are summarized in Table 5-8.

Table 5-8. Results of using the profit sharing mechanism Scenario 1

\begin{tabular}{ccccccccc}
\hline Scheme & $T C_{s}(\$)$ & $\frac{T C_{v}}{T C_{s}}(\%)$ & $\frac{T C_{b}}{T C_{s}}(\%)$ & $\begin{array}{c}\text { Adjusted } \\
\text { vendor's profit } \\
(\$)\end{array}$ & $\begin{array}{c}\text { Adjusted } \\
\text { buyer's profit } \\
(\$)\end{array}$ & $\begin{array}{c}\text { Vendor's ROI } \\
\text { after adjustment } \\
(\%)\end{array}$ & $\begin{array}{c}\text { Buyer's ROI } \\
\text { after adjustment } \\
(\%)\end{array}$ & $\begin{array}{c}\sigma \\
\sigma\end{array}$ \\
\hline 1 & $12,140.3$ & 42.9 & 57.1 & 601.7 & 800.8 & 11.6 & 11.6 & 140.8 \\
2 & $12,033.8$ & 45.4 & 54.6 & 448.1 & 539.9 & 8.2 & 8.2 & 64.9 \\
3 & $11,969.5$ & 45.0 & 55.0 & 465.2 & 568.4 & 8.6 & 73.0 \\
4 & $12,155.4$ & 42.7 & 57.3 & 575.9 & 772.8 & 11.1 & 11.1 & 139.2 \\
\hline
\end{tabular}

(TC: total cost, TP: total profit; subscripts $v=$ vendor, $b=$ buyer and $s=$ system)

The results in Table 5-8 show that the vendor's adjusted profits in dollars are equal to 601.7, 448.1, 465.2 and 575.9 with ROIs of $11.6 \%, 8.2 \%, 8.6 \%$ and $11.1 \%$ for Scheme 1, 2, 3 and 4, respectively. The buyer's profits in dollars are reduced and equal to 800.8, 539.9 and 568.4 for Scheme 1, 2 and 3, respectively, where it is increased for Scheme 4 to 772.8 with the same ROIs of the vendor.

Comparing the results in Table 5-3 with those in Table 5-8, one can realize that the buyer's profit in Scheme 1 before using the equal ROI profit sharing mechanism is equal to $\$ 848.2$ where it reduces to $\$ 800.8$ after the adjustment. Therefore, the buyer pays the vendor $\$ 47.4$, which increases its total profit to $\$ 601.7$ from $\$ 554.2$. For Scheme 2 and 3, the buyer pays the vendor \$422.0 (961.9-539.9) and \$351.1 (919.5-568.4), respectively. On the other hand, in Scheme 4, the vendor pays the buyer $\$ 0.9$ (almost nothing) which increases the buyer's profit to $\$ 772.8$.

The values of the standard deviation $(\sigma)$ in Table 5-8 are less than those in Table 5-7 because of using this profit sharing mechanism. As shown in Table 5-7 and Table 5-8, the standard deviation for Scheme 1 before using the profit sharing mechanism is 207.9 where it is 140.8 after sharing 
the profit. Also, the standard deviation is decreased from 661.7 and 569.5 to 64.9 and 73.0 for Scheme 2 and 3, respectively. On the other hand, it is increased slightly by 1.2 for Scheme 4. Therefore, the results above show that using the profit sharing mechanism helps reduce the difference between the profits of the players in the chain.

\subsubsection{Scenario 2: Increase in total cost resulted from adopting the CS}

This scenario assumes that the buyer and the vendor work under a non-coordinated system. Hence, the buyer orders a quantity $\left(q^{*}\right)$ that maximizes its profit and the vendor, which has a small warehouse and cannot carry a large size inventory, uses the lot-for-lot policy to produce and ship the items. Also, the buyer pays the vendor when it receives the shipment. Additionally, it is assumed that the vendor wants to change its policy and adopt the CS policy to get some benefits from using its buyer's warehouse. Therefore, it needs to convince the buyer to change its decision and adopt the CS. To do so, the vendor needs to make sure that the buyer's profit increases or remains the same as a result of the CS, or compensate it if the profit is decreased. Therefore, in this scenario, the vendor pays the buyer the difference between what it used to make before and after adopting the CS policy. In addition, the vendor also pays the buyer a percentage of the system's profit, which is equal to the increase in the total cost caused by adopting the CS policy.

The buyer's total profit in the non-coordinated system $\left(T P_{b}^{n c}\right)$ is calculated such as

$$
T P_{b}^{n c}=\left(c_{c}-c_{b}\right) D-\left(O_{b}+c_{t}\right)\left(\frac{D}{q}\right)-h_{b} \frac{q}{2}
$$

The term $c_{c} D$ represents the annual revenue generated from selling the products, $c_{b} D$ for the annual purchasing cost, $O_{b}(D / q)$ for the annual ordering cost, $c_{t}(D / q)$ for the annual transaction cost, and $h_{b}(q / 2)$ is the annual holding cost. By taking the second derivative for the above equation, it was found that it is negative for all values of $q$ indicating that Eq. (5-24) is concave. The optimal quantity for the non-coordinated system $\left(q_{n c}^{*}\right)$ is found by setting the first derivative for the total system profit equal to zero, and solving for $q$. The optimal quantity is equal to

$$
q_{n c}^{*}=\sqrt{\frac{2\left(O_{b}+c_{t}\right) D}{h_{b}}}
$$


The vendor's total profit in the non-coordinated system $\left(T P_{v}^{n c}\right)$ consists of the annual revenue it makes from selling the items to the buyer, $\left(c_{b} D\right)$, less its annual total cost, which includes the purchasing cost for the raw material, $\left(c_{p} \gamma D\right)$, the production cost, $\left(c_{p r} D\right)$, the set-up cost, $S_{v}(D / q)$, and the holding cost, $h_{v}(q / 2)$. Therefore, the annual total profit is written as

$$
T P_{v}^{n c}=\left(c_{b}-\gamma c_{p}-c_{p r}\right) D-\left(S_{v}\right)\left(\frac{D}{q}\right)-h_{v} \frac{q}{2}
$$

Using the same numbers that are used in the numerical example and plugging them into Eq. (5-25), the optimal quantity for the buyer is given in Table 5-9.

Table 5-9. Results of the non-coordinated system

\begin{tabular}{cccccc}
\hline$i$ & $q_{n c}^{*}$ & $T C_{i}^{n c}(\$)$ & $T R_{i}^{n c}(\$)$ & $T P_{i}^{n c}(\$)$ & ROI (\%) \\
\hline$v$ & & $8,162.6$ & $5,400.0$ & $-2,762.6$ & $-33.8 \%$ \\
$b$ & 101 & $5,905.0$ & $7,290.0$ & $1,385.0$ & $23.5 \%$ \\
\hline
\end{tabular}

(TC: total cost, $T P$ : total profit, TR: total revenue; subscripts $v=$ vendor and $b=$ buyer)

Table 5-9 shows that the optimum quantity $\left(q_{n c}^{*}\right)$ that maximizes the buyer's profit $\left(T P_{i}^{n c}\right.$ where $\left.i=b\right)$ is 101 units. The buyer's total cost $\left(T C_{b}^{n c}\right)$ is calculated by excluding the term $c_{c} D$ from Eq. (5-24), which is equal to $\$ 5,905.0$. The total revenue for the buyer, which is equal to $\$ 7,290.0$, is calculated such that $T R_{b}^{n c}=c_{c} D$, and its profit is found by subtracting the buyer's total cost from its revenue, or by using Eq. (5-24). The vendor's total cost $\left(T C_{i}^{n c}\right.$ where $\left.i=v\right)$, which is equal to $\$ 8,162.6$, is calculated by excluding its total revenue $\left(T R_{v}^{n c}=c_{b} D=\$ 5,400.0\right)$ from Eq. (5-26), and using the optimum quantity for the buyer $\left(q^{*}\right)$ as it uses the LFL policy. Accordingly, the total profit for the vendor $\left(T P_{v}^{n c}\right)$ is equal to its revenue less its total cost, or can be calculated using Eq. (5-26).

The results for the non-coordinated system, as seen in Table 5-9, show that the buyer makes $\$ 1,385.0$ as a profit where the vendor loses $\$ 2,762.6$ instead of making a profit. Comparing the results to those in Table 5-3, one can realize that by using the CS policy, the vendor makes a profit where the buyer loses a proportion of its profit in the non-coordinated system. As mentioned above, the buyer adopts the CS policy if it yields at least the return as the non-coordinated case. Therefore, to meet this condition, the vendor compensates the buyer for any loss incurred from adopting the CS agreement, which is equal to $\left(T P_{b}^{n c}-T P_{b}\right)$ where $T P_{b}^{n c}$ is provided in Table 5-9 and $T P_{b}$ 
values for Schemes 1, 2, 3, and 4 are found in Table 5-3. In addition, to fortify coordination, the vendor is assumed to pay the buyer a share of the profits generated (clustered with vendor) from adopting the CS agreement, which is equal to the percentage increase in the buyer's total cost $\left(T C_{b}-T C_{b}^{n c}\right) T P_{s} / T C_{b}^{n c}$. The buyer's total cost when the CS is adopted $\left(T C_{b}\right)$ is given in Table 5-3 where its total cost for the non-coordinated system $\left(T C_{b}^{n c}\right)$ is given in Table 5-9. By applying the two steps above to Table 5-3, the results for the buyer's and the vendor's adjusted profit are given in Table 5-10.

Table 5-10. Results when using the profit sharing mechanism Scenario 2

\begin{tabular}{ccccc}
\hline Scheme & $\left(T P_{b}^{n c}-T P_{b}\right)(\$)$ & $\frac{\left(T C_{b}-T C_{b}^{n c}\right) T P_{s}}{T C_{b}^{n c}}(\$)$ & $\begin{array}{c}\text { Adjusted buyer's } \\
\text { profit }(\$)\end{array}$ & $\begin{array}{c}\text { Adjusted vendor's profit } \\
(\$)\end{array}$ \\
\hline 1 & 536.8 & 243.8 & $1,628.9$ & -226.4 \\
2 & 423.1 & 112.3 & $1,497.3$ & -509.3 \\
3 & 465.6 & 118.6 & $1,503.6$ & -470.0 \\
4 & 613.1 & 242.2 & $1,627.2$ & -278.5 \\
\hline
\end{tabular}

Table 5-10 represents the results for using the profit sharing Scenario 2. The results show that the vendor loses \$226.4 in Scheme 1 when the CS policy is adopted with the profit sharing Scenario 2 instead of losing $\$ 2,762.6$ when the system is non-coordinated. Moreover, the results show that the vendor reduces its loss from $\$ 2,762.6$ to $\$ 509.3, \$ 470.0$, and $\$ 278.5$ for Schemes 2,3 and 4 , respectively. At the same time, the buyer's ROI remains similar to the one in the non-coordinated system, which is equal to $23.5 \%$. According to the results above, the buyer maintains its ROI, and the vendor reduces its loss when the CS with profit sharing Scenario 2 is adopted. This helps the vendor to convince the buyer to adopt the new policy and save money.

\subsubsection{Scenario 3: Equal distribution of the excess profit}

For this mechanism, the players make 50\% more profit from adopting the CS policy than what they make from classical coordination. This scenario assumes that the vendor and the buyer work under a coordinated system (adopting the model of Hill, 1999), but they want to change the working policy and adopt the CS instead. Thus, it is necessary to compare the results of the model of Hill (1999) with the results of the CS model (Table 5-3). To do that, Eq. (1) of Hill's model (1999) is adopted and modified to be a profit rather than a cost function. Therefore, other annual costs and revenue factors are added to the equation such as the purchasing cost of raw material $\left(c_{p} \gamma D\right)$, the production cost $\left(c_{p r} D\right)$, the buyer's purchasing $\operatorname{cost}\left(c_{b} D\right)$, the vendor's revenue $\left(c_{b} D\right)$ 
and the buyer's revenue $\left(c_{c} D\right)$. In a classical coordinated system, the buyer pays the vendor when it receives a shipment. Therefore, it incurs a transaction cost $\left(n c_{t} D / q\right)$. The total profit for the system is equal to the sum of the revenues that the players make minus their costs. The annual system's profit for Hill's model, after some modifications, is written as

$$
\begin{aligned}
& T P^{H i l l}=\left(c_{c}-\gamma c_{p}-c_{p r}\right) D-\left(S_{v}+n O_{b}+n c_{t}\right)\left(\frac{D}{n q}\right)-h_{v}\left(\frac{q D}{P}+\frac{(P-D) n q}{2 P}\right)-\left(h_{b}-\right. \\
& \left.h_{v}\right) \frac{q}{2}
\end{aligned}
$$

By taking the second derivative for the above equation, it was found that it is negative for all values of $q$ indicating that Eq. (5-27) is concave. The optimal quantity $\left(q_{h}^{*}\right)$ was found by setting the first derivative for the total system profit equal to zero and solving for $q$. The optimal quantity is written as

$$
q_{h}^{*}=\sqrt{\frac{\left(S_{v}+n O_{b}+n c_{t}\right)\left(\frac{D}{n}\right)}{h_{v}\left(\frac{D}{P}+\frac{(P-D) n}{2 P}\right)+\frac{\left(h_{b}-h_{v}\right)}{2}}}
$$

Using the equations above with the same numbers considered in the numerical example, the results for the model of Hill (1999) after the modification are shown in Table 5-11.

Table 5-11. Results for Hill's model

\begin{tabular}{ccccccc}
\hline$n$ & $i$ & $q_{h}^{*}$ & $T C_{i}^{\text {Hill }}(\$)$ & $T R_{i}^{\text {Hill }}(\$)$ & $T P_{i}^{\text {Hill }}(\$)$ & ROI Hill $\left._{i}^{\text {Hil }}\right)$ \\
\hline \multirow{3}{*}{4} & $v$ & & $5,385.0$ & $5,400.0$ & 15.0 & 0.3 \\
& $b$ & 132 & $5,922.7$ & $7,290.0$ & $1,367.3$ & 23.1 \\
& \multirow{2}{*}{$s$} & & $11,307.7$ & $1,2690.0$ & $1,382.3$ & 12.2 \\
\hline
\end{tabular}

(Subscripts $i, v=$ vendor, $b=$ buyer and $s=$ system)

Table 5-11 shows that the optimum quantity $\left(q_{h}^{*}\right)$ that maximizes the system's profit, using Hill's model, is 132 units. The vendor's annual total cost is equal to the raw material purchasing cost $\left(c_{p} \gamma D\right)$, the production cost $\left(c_{p r} D\right)$, the set-up cost $\left(S_{v} D / n q\right)$, and the holding cost $\left(h_{v}(q D / P+(P-D) n q / 2 P-q / 2)\right)$, which is obtained from Eq. (5-27). The annual revenue for the vendor is equal to $c_{b} D$. The buyer's annual total cost, from Eq. (5-27), is equal to the buyer's purchasing cost $\left(c_{b} D\right)$, the ordering cost $\left(O_{b} D / q\right)$, the transaction cost $\left(c_{t} D / q\right)$, and the holding cost $\left(h_{b} q / 2\right)$. The buyer's annual revenue from selling the products is equal to $c_{c} D$. 
The results show that using Hill's model is more profitable than adopting the CS agreement with Schemes 2, 3 and 4 (see Table 5-12). As a result, both players do not adopt the CS policy that works under these three payment schemes, and the profit sharing Scenario 3 is not applicable (NA). On the other hand, this profit sharing scenario can be applied to the CS Scheme 1 as it is more profitable than Hill's model. The difference between $T P_{S}$ for Scheme 1 (in Table 5-3) and $T P_{S}^{\text {hill }}$ (in Table 5-11) returns a $\$ 20.2$ excess profit, which is distributed among the players (50-50\%) as described earlier. Therefore, the buyer's adjusted profit is equal to the profit generated when working under Hill's policy $\left(T P_{b}^{\text {Hill }}\right)$, found in Table 5-11, in addition to its proportion from the excess profit ( $0.5 *$ excess profit) as seen in Table 5-12. Also, the vendor's adjusted profit is equal to the profit generated when working under Hill's policy $\left(T P_{v}^{H i l l}\right)$, found in Table 5-11, in addition to its proportion from the excess profit ( $0.5 *$ excess profit) as seen in Table 5-12. The outcome for this scenario shows that both players and the system make higher profits (see Table 5-12) compared to Hill's model (see Table 5-11), but the ROIs for the system and the buyer are decreased, which are resulted from increasing the buyer's total cost. Comparing the results before and after using this profit sharing mechanism, the ROI for the system is decreased from $12.2 \%$ to $11.6 \%$ where it is decreased from $23.1 \%$ to $19.9 \%$ for the buyer. Thus, the buyer refuses to adopt the CS policy and prefer to work under Hill's policy.

Table 5-12. Results of using the profit sharing mechanism Scenario 3

\begin{tabular}{|c|c|c|c|c|c|c|c|}
\hline Scheme & $T P_{S}(\$)$ & $\begin{array}{l}T P_{S}^{\text {Hill }} \\
\quad(\$)\end{array}$ & $\begin{array}{l}\text { Excess } \\
\text { profit }(\$)\end{array}$ & $\begin{array}{c}\text { Buyer adjusted } \\
\text { profit }(\$)\end{array}$ & $\begin{array}{c}\text { Vendor adjusted } \\
\text { profit }(\$)\end{array}$ & $\begin{array}{c}\text { Buyer } \\
\text { ROI }(\%)\end{array}$ & $\begin{array}{l}\text { Vendor } \\
\text { ROI (\%) }\end{array}$ \\
\hline 1 & $1,402.5$ & $1,382.3$ & 20.2 & $1,377.4$ & 25.1 & 19.9 & 0.5 \\
\hline 2 & 988.0 & $1,382.3$ & -394.3 & NA & NA & NA & NA \\
\hline 3 & 1,033.6 & $1,382.3$ & -348.7 & NA & NA & NA & NA \\
\hline 4 & $1,348.7$ & $1,382.3$ & -33.6 & NA & NA & NA & NA \\
\hline
\end{tabular}

The first two profit sharing scenarios show that it is better to incorporate a profit sharing mechanism when working under the CS policy. There are two reasons behind incorporating the profit sharing mechanism, which are playing a major role in the decision of the player as well as reducing the variance between the profits of the players in the system (see for example Scenarios 1 and 2). On the other hand, Scenario 3 shows that using Hill's model is better than incorporating the profit sharing mechanism with the CS policy. Although the total profits are increased for both 
players as well as for the system, the ROIs for the system and the buyer are decreased. Therefore, the vendor has to choose the profit sharing mechanism that works for the favor for both players, and returns higher profits and ROIs to convince the buyer to change its decision.

\subsection{Sensitivity Analysis}

This section presents the results of the sensitivity analysis to show the effects of changing different parameters on the developed payment schemes models. When changing a set of parameters, the values of the other parameters in Table 5-2 are kept unchanged. The following tests were performed:

1. Varying the ratio of the demand rate to the production rate, $D / P$ : As $D / P$ increases (from 0.3 to 0.9 ), the supply chain profit was shown to increase for the four schemes. The equal payment scheme (\#1) was shown to be the most profitable. (See Figure 5-5)

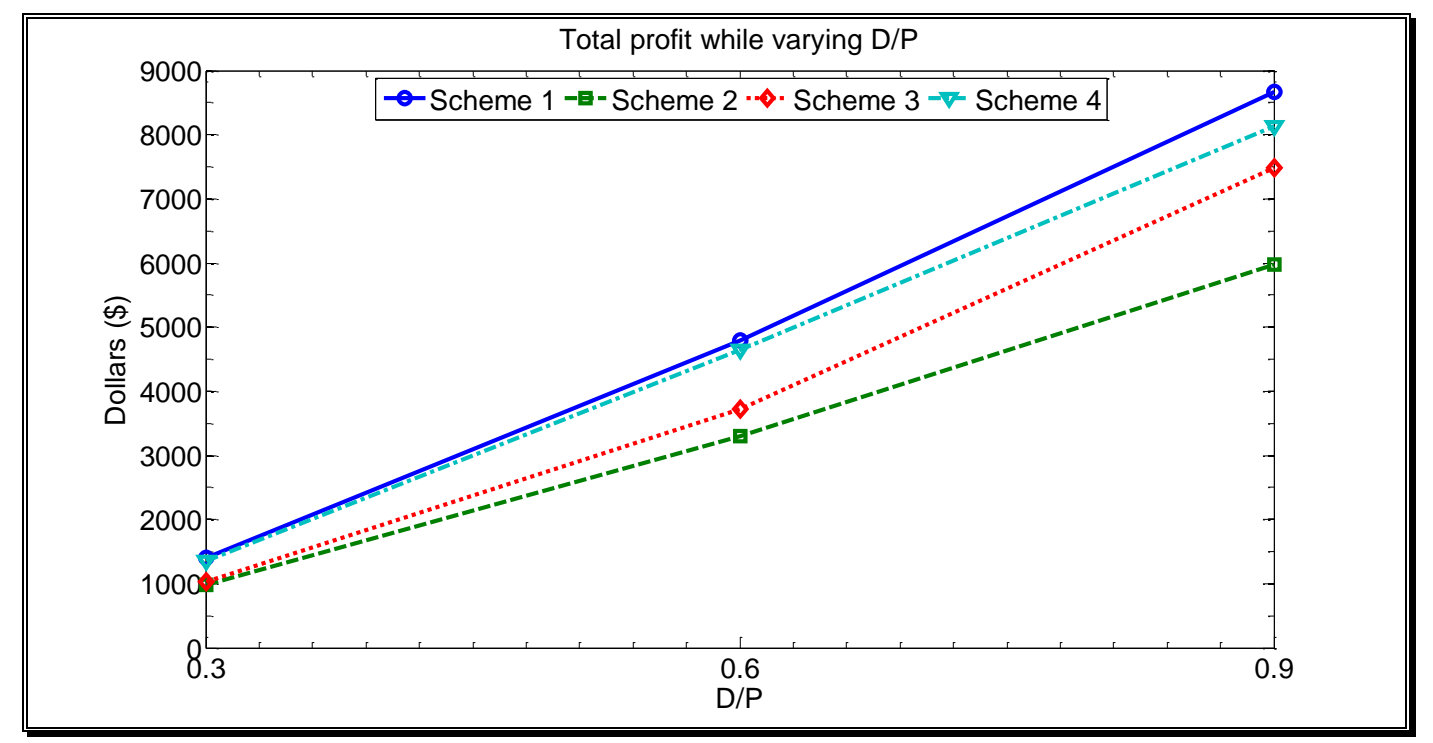

Figure 5-5. Total profit while varying the ratio of $D / P$ for all payment schemes

2. Varying the ratio of the interest rates, $I_{b} / I_{v}$ : As the interest rate of the buyer increases relative to that of the vendor (from 0.5 to 3 ), the total profit for the supply chain increases. The equal payment scheme (\#1) was shown to be the most profitable, followed by Scheme 4 of two unequal payments with the smallest at the end. The least profitable scheme is the one where the buyer pays upon receiving each shipment. (See Figure 5-6 next page) 


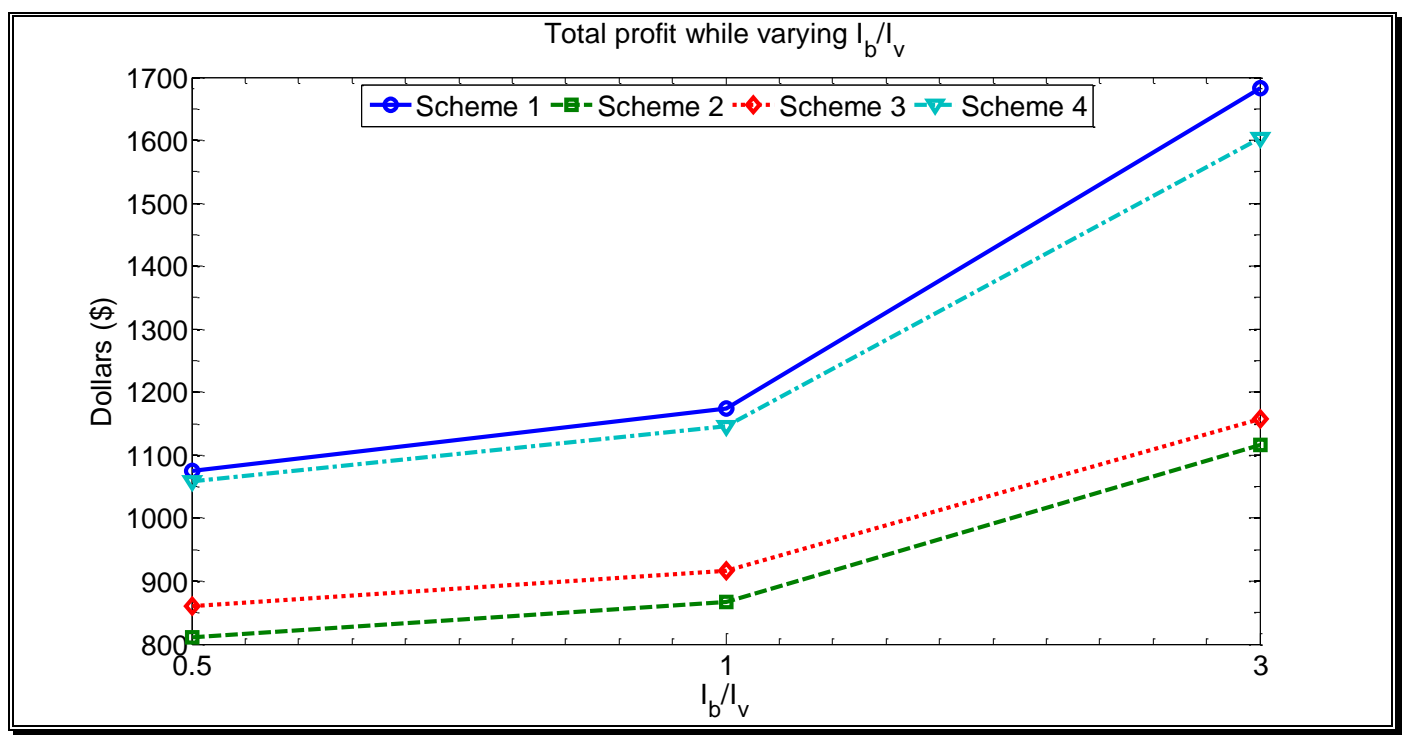

Figure 5-6. Total profit while varying the ratio of $I_{b} / I_{v}$ for all payment schemes

3. Varying the ratio of the selling prices, $c_{c} / c_{b}$ : The results showed that as $c_{c} / c_{b}$ increases (from 1 to 2.3), Scheme 1, equal payment, and Scheme 4, two unequal payments with the smallest at the end, were shown to be more profitable than Schemes 2 and 3, with Scheme 1 slightly better than Scheme 4. The results showed that when $c_{c}$ is increased to $\$ 31.3$, the number of payments in Scheme 1 changes from $m=1$ to $m=2$ with $q^{*}=129.9, n=11$ and the system total profit was found to be equal to $\$ 26,058.6$. (See Figure 5-7)

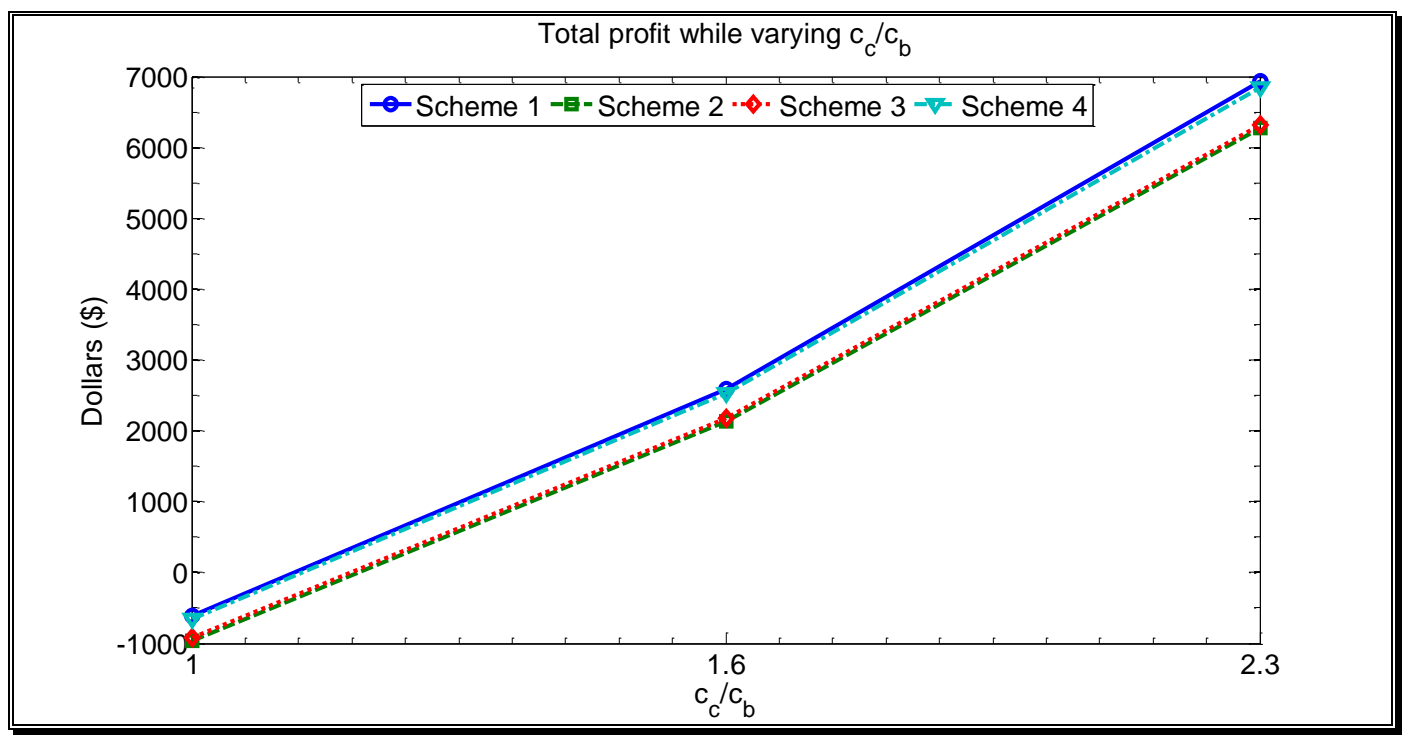

Figure 5-7. Total profit while varying the ratio of $c_{c} / c_{b}$ for all payment schemes 
4. Varying the ratio of the holding costs, $h_{b} / h_{v}$ : The results showed that as $h_{b} / h_{v}$ increases (from 0.5 to 1.5 ), Scheme 1 was shown to be the most profitable of the four schemes, with Scheme 4 being the second and Scheme 2 being the last. The results showed that the most profits were recorded for all schemes when the holding cost at the buyer is less than that at the vendor. (See Figure 5-8)

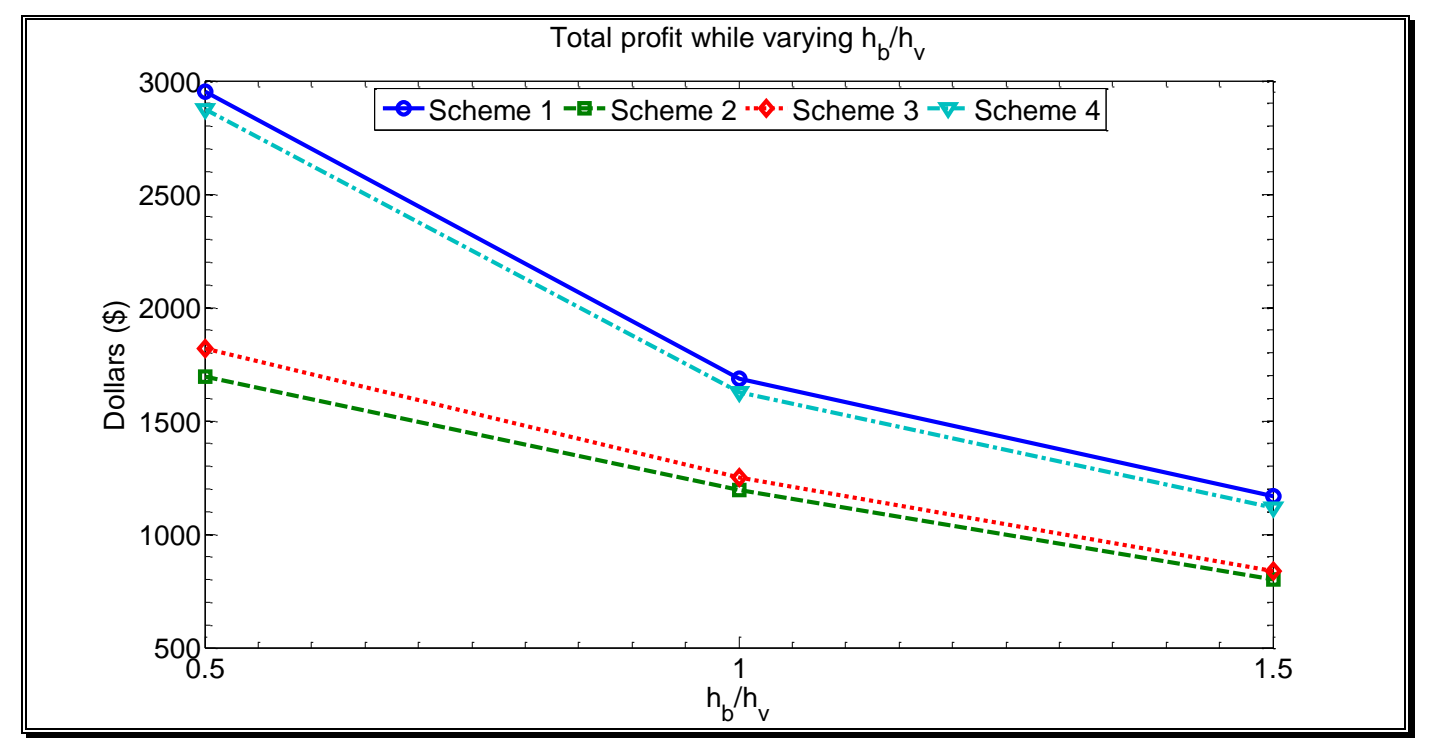

Figure 5-8. Total profit while varying the ratio of $h_{b} / h_{v}$ for all payment schemes

5. Varying the ratio of the ordering cost of the buyer to the setup cost of the vendor, $O_{b} / S_{v}$ : The results showed that Scheme 1, equal payments, was the most profitable of the other schemes when increasing $O_{b} / S_{v}$ (from 0.1 to 0.6 ). However, the supply chain profits decreased for all scenarios as $O_{b} / S_{v}$ increases. Comparing Scheme 2 with Scheme 3, the results showed that when $O_{b} / S_{v} \leq 0.11$, Scheme 3 returned better results where Scheme 2 returned a higher profit when $O_{b} / S_{v}>0.11$. When $O_{b} / S_{v}$ increases, the optimum order quantity and the number of shipments are affected, which affects the setup, holding, capital, ordering, and transaction costs and the revenue from investing sales. The results showed that increasing $O_{b} / S_{v}$ from 0.1 to 0.3 , increased the total costs, which depends on $n^{*}$ and $q^{*}$, by $22 \%$ and $20 \%$ for Scheme 3 and Scheme 2, respectively. (See Figure 5-9 next page) 


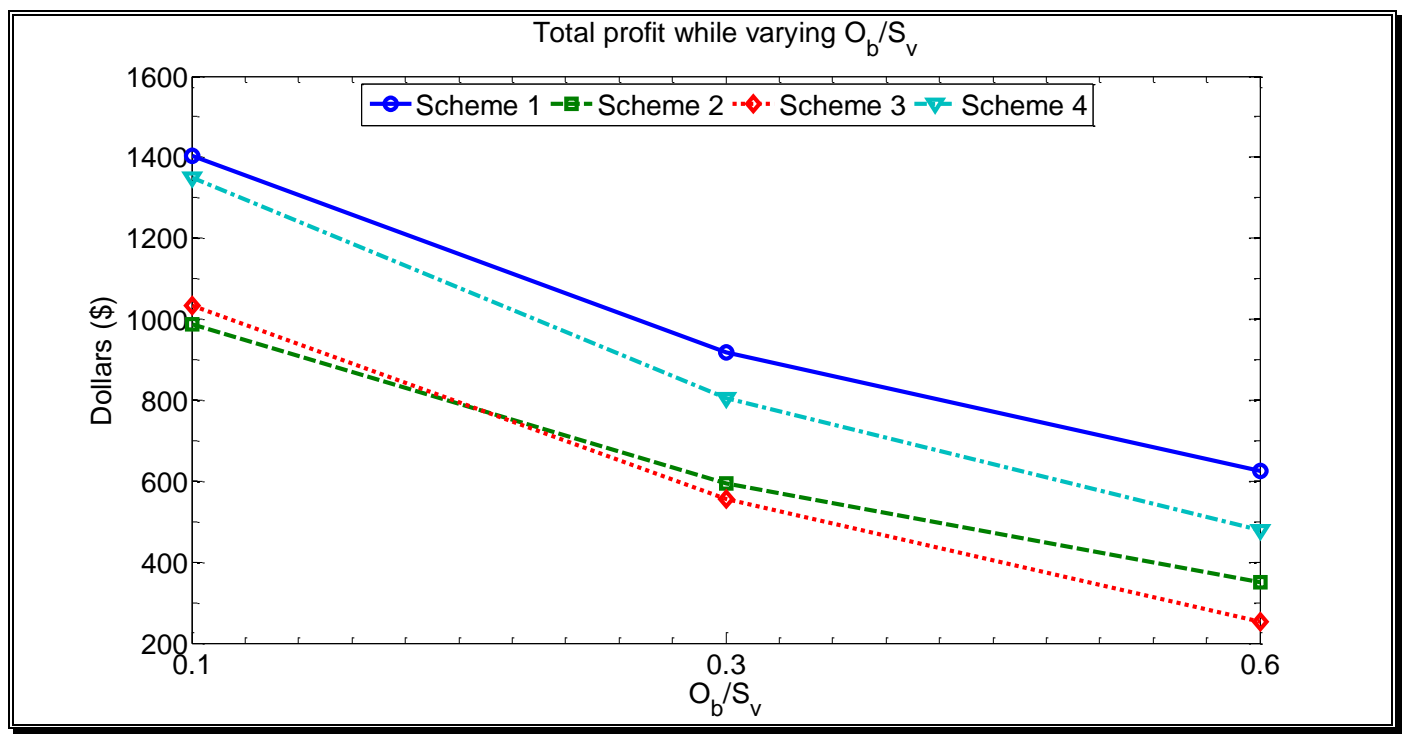

Figure 5-9. Total profit while varying the ratio of $O_{b} / S_{v}$ for all payment schemes

6. Other parameters, like the ratio of the transaction cost to the buyer's selling price $c_{t} / c_{c}$ and the ratio of the buyer's to the vendor's audit $\operatorname{costs} c_{b}^{a} / c_{v}^{a}$, were varied and again Scheme 1 was shown to be the most profitable. (See Figure 5-10 and Figure 5-11 next page)

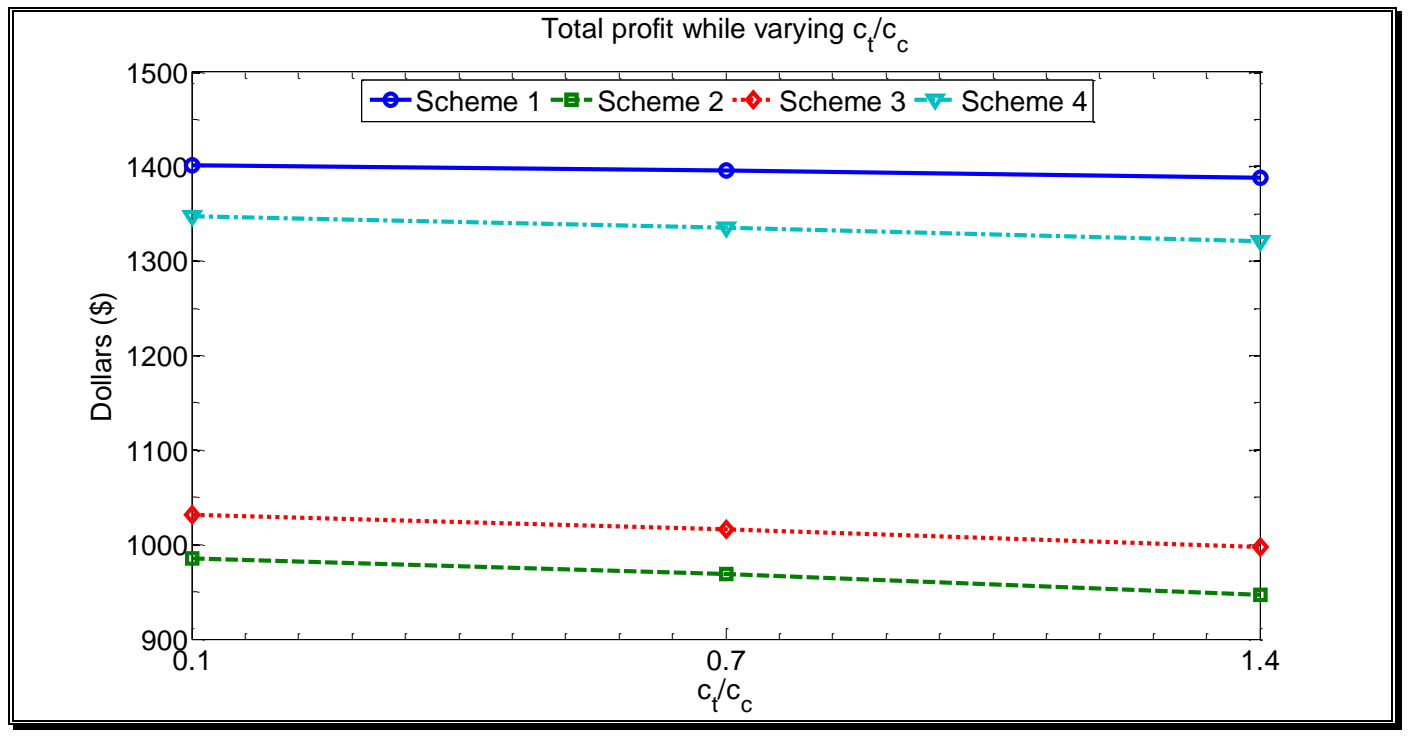

Figure 5-10. Total profit while varying the ratio of $c_{t} / c_{c}$ for all payment schemes 


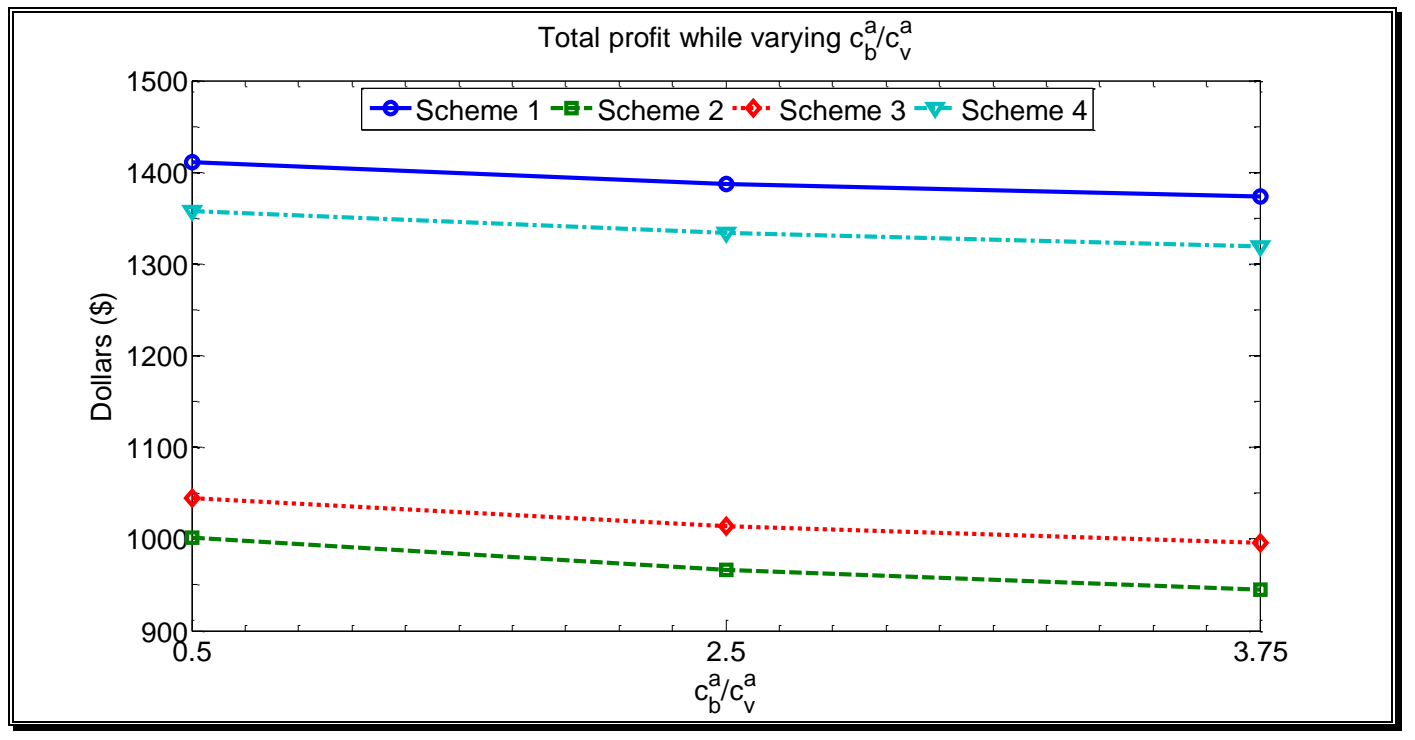

Figure 5-11. Total profit while varying the ratio of $c_{b}^{a} / c_{v}^{a}$ for all payment schemes

\subsection{Managerial Insights}

This section provides general managerial insights to help managers and/or decision makers to make the right decision.

1. Consignment stock agreement benefits the vendor as it reduces its storage and handling costs, frees its space, and helps in selling slow moving items that the buyer does not want to buy.

2. Consignment stock ensures products availability on shelves at the buyer's side, which results in a better display. This helps in increasing product's demand and system's profit.

3. Reducing the number of invoices and payments helps the system to generate more profits.

4. Although reducing the number of payments and delaying it to the end of the cycle increases the profit of the system, financial department has to set a maximum time limit in which the buyer has to be invoiced, so that the vendor is not affected financially.

5. A scheme of equal-sized payments at equal time intervals is more profitable and practical as it helps in financial management for both the vendor and the buyer.

6. Having an information system may help players monitor the sales and reduce the number of audits required per year, and reduce the system's cost. 
7. Profit sharing can make a CS contract more profitable and appealing to players.

\subsection{Conclusion}

This chapter aimed at investigating the effects of different payment schemes on the total profit of a two-level (a vendor and a buyer) supply chain system when the CS agreement is adopted as a coordination mechanism. Then, the chapter compared between different payment schemes, some of them were similar to what is practiced, to show which of the schemes is the most profitable for the system and under what conditions.

Numerical results showed that the system, in particular the vendor, benefits from adopting the CS agreement. In general, it increases its profit or sometimes reduces its loss. The results also showed that the number of payments and its relative cost affect both the order policy and the total profit of the system. For instance, the optimum quantity and the total supply chain profit decrease when the number of payments increases. The reason behind this is that the annual ordering cost of the buyer increases when the quantity per shipment decreases. Moreover, by analyzing the results of the numerical example, one can realize that it is always better to reduce the number of payments and keep them to the end of the cycle. This helps in increasing the profit of the system, especially the buyer by giving it more time to invest the money that is generated from selling the products before paying the vendor and make more profits. As the CS always increases the buyer's total cost,

specifically the holding cost (see, Braglia and Zavanella, 2003), allowing it to invest the money before paying for the used/withdrawn items helps in covering some of the expenses, which are caused by adopting this type of agreement. In addition, this may convince the buyer to accept the CS agreement particularly if the revenue from the investment is too high. Furthermore, the results of the numerical example showed that the scheme that is in favor of the player or the system is different. For example, the buyer generates the highest profit if Scheme 2 was used where Scheme 4 was shown to be better for the vendor. However, Scheme 1 was shown to be the most profitable for many instances.

In addition to the numerical example that was adopted from Braglia and Zavanella (2003), 2,000 random numerical examples were generated, and the optimum solutions were found for each payment scheme of these examples. The aim of generating and solving numerous random examples than focusing on one practical example was to have a general view of the behavior of 
the developed models. The results of the 2,000 examples confirmed that Scheme 1 always returned the highest total profit among the four schemes. In addition, they showed that paying the vendor one payment at the end of the cycle was always better than making frequent small payments. Moreover, the analysis of these examples showed that Scheme 4 recorded the second highest total profit of the system with minor difference than Scheme 1 in $86.65 \%$ of the total examples that were solved. The third highest total profit for the system was generated by Scheme 2 in $78.45 \%$ of the 2,000 examples, where Scheme 3 returned the least total system profit in $82.7 \%$ of these examples. Thus, the analysis of results showed that choosing the best payment scheme depends on the values of the input parameters.

The comparison between the results of the numerical example and the model of Braglia and Zavanella (2003), after modifying their equation to calculate the total profit, showed that although they had better results, the reality of the CS contract was not reflected as they did not consider the revenues for investment and the additional cost factors that were adopted from real CS contracts. On the other hand, modifying Scheme 1 to reflect what was claimed by Braglia and Zavanella (2003), the vendor is paid daily, showed the least total profit and at the same time it is impractical. This confirms that increasing the number of payments highly affects the total profit of the system as it increases its relative cost. Thus, reducing it is recommended. Moreover, the results demonstrated that finding the optimal number of payments is required to enhance the performance of the system, and its total profit.

Since adopting the CS benefits the vendor and reduces its total cost while reducing the total profit of the buyer, which plays a major role in its decision of accepting the CS policy, three different profit sharing scenarios were applied as examples. The results showed that incorporating the profit sharing with the CS policy has a positive impact on the system. The results of the first scenario showed that in most cases the variance between the profits of the vendor and the buyer is reduced. In the second profit sharing scenario, the numbers showed that the vendor can convince the buyer to accept the CS agreement. Although this agreement always reduces the buyer's ROI and profit, Scenario 2 showed that incorporating the profit sharing mechanism helps in maintaining them which plays a major role on the decision of the buyer, and reduces the vendor's loss. The results for the last profit sharing scenario showed that adopting the model of Hill (1999) is better than the CS policy although the ROI of the vendor was increased slightly. The reason is that the buyer's 
ROI reduces by $3.2 \%$ if it accepts the CS, and sign the contract as the CS always increases its costs especially the holding cost. The only way that the CS with this profit sharing scenario is better than Hill's model is when the buyer cannot predict its demand because of the variation in the market. In this case, the CS helps the buyer to satisfy its customers and not losing sales, which affects its reputation if the product was not found on the shelf continuously.

In addition to the numerical example and the profit sharing scenarios, a sensitivity analysis was performed to test the effect of varying some of the parameters on the models. The results of the sensitivity analysis showed that changing some of the parameters, such as the buyer's ordering cost, affect choosing the right payment scheme to be adopted. As a result, choosing the best payment scheme that maximizes the total profit of the system depends on the exact value of the parameters, but in most cases making one payment at the end of the cycle is more profitable and gives better results.

Moreover, managerial insights were provided to assist managers and decision makers in recognizing the benefits of a consignment stock agreement. They also show which payment is more practical and what might affect the profit of the system.

This study could consider deterministic or stochastic lead-time of shipments, which affects the inventory policy and the payment scheme. Assuming a stochastic demand will add more complexity to the models making EOQ model not suitable. In addition, one can study the effect of the delay in payment, from the buyer side, on the total profit of the system. Moreover, considering a human error in managing the products as a cost is another extension of the proposed model. Finally, one can extend the models in this chapter by looking at different payment schemes or payment methods (i.e. in cash or credit) or studying the effect using a net present value approach to modeling the problem.

The next chapter studies the effect of offering a delay-in-payment to the buyer to settle down its account when a CS agreement is adopted between the players. 


\section{Chapter 6: The consignment stock case for a vendor and a buyer with delay-in-payments}

Supply Chain Management (SCM) is a complex topic that involves managing several functional areas within a supply chain system to improve its performance indicators. Different models have been developed to manage inventories and solve related issues (i.e. logistics and transportation). Proper inventory management (IM) helps in increasing the profit (lowering the cost) of a supply chain system. This led many researchers to focus on developing and investigating different inventory models and supply chain coordination mechanisms that represent different inventory situations (e.g., Bushuev et al., 2015) and supply chain structures of two or more levels with single or multiple entities at each level (see Jaber and Zolfaghari, 2008; Glock, 2012). One of the coordination mechanisms that have caught the attention of researchers is CS, which is the focus of this chapter.

The surveyed works on consignment stock (CS) implicitly adopted the assumption of Braglia and Zavanella (2003) that the buyer pays the vendor as items are withdrawn from inventory, an unrealistic and impractical assumption. Chapter 5 studied the effect of different payment schemes on the total profit of a supply chain system with CS. It was found that a scheme of equal-sized payment made at equal time intervals is the most profitable and practical for the system. The investigation of several CS business contracts, of which several are available online (see i.e. Table 6-1 next page, and Table 10-1 in Appendix 1), showed that a payment scheme coupled with delay-in-payments is practiced by some firms, and is offered by the vendor to its buyers where it charges interest on balances past the payment due date. For instance, it is mentioned in one of the terms of LBMA's contract that the consignor (vendor) has the right to charge the consignee (buyer) an interest on balances not settled by the due date. Also, it is mentioned in the Trelleborg's contract that the vendor invoices the buyer for products purchased in the previous month, and the due date of the payment is 30 days from the invoice date. This is a real example that an equal interval invoice scheme and delay-in-payments are jointly practiced. One more example is that of CARFAC Saskatchewan Visual Artists in which the dealer has to settle down its payment within 14 days of the time of the invoice. Otherwise, $12 \%$ interest rate is charged on any overdue amount until the payment is made. Moreover, in The Newcastle upon Tyne Hospitals NHS Foundation Trust contract, it is stated that the customer has to settle down its account in full within 30 days 
from the time the customer receives the invoice in order to replenish its CS warehouse. Stone Art Manufacturing contract mentions that the payment has to be made within 7 days of the invoice date, and interest is charged for the unpaid amount. The last example in this chapter is the one for NASCO Distributer Sales that allows its customer 45 days from the day of the invoice to pay. Table 6-1 lists few real CS contracts (exact contract closes are found in Appendix 1).

Table 6-1. Samples of real consignment stock contracts

\begin{tabular}{ll}
\hline Name of the company & Reference to the CS contract \\
\hline LBMA & $\underline{\text { http://www.lbma.org.uk/assets/LBMA\%20Consignment\%20Agreement\%2020051219.pdf }}$ \\
Trelleborg & $\begin{array}{l}\text { http://www.trelleborg.com.au/wp-content/uploads/2013/08/TESA-Terms-of-Sale-on- } \\
\text { Consignment.pdf }\end{array}$ \\
CARFAC Saskatchewan & $\underline{\text { http://www.carfac.sk.ca/assets/Consignment Agreement 2010-05-13.pdf }}$ \\
Visual Artists & \\
The Newcastle upon Tyne & $\underline{\text { http://www.newcastle- }}$ \\
Hospitals NHS & $\underline{\text { hospitals.org.uk/downloads/policies/Operational/ConsignmentStockPolicyandProcedure20 }}$ \\
Foundation Trust & $\underline{\text { hto7.pdf }}$ \\
Stone Art Manufacturing & $\underline{\text { htt//www.stoneartmanufacturing.co.za/My_Homepage_Files/Download/Consignment\%2 }}$ \\
NASCO Distributor Sales & $\underline{\text { http://www.nascosales.com/assets/downloads/cosignment_agreement.pdf }}$ \\
\hline
\end{tabular}

Although delay-in-payments has been practiced by firms adopting CS, to the best of the author's knowledge, no study has yet investigated the effect of delay-in-payments on a supply chain system with CS agreement. Therefore, this chapter focuses on addressing one more gap in the CS literature. It builds on the work of Chapter 5 by investigating the joint effect of permissible delayin-payments and making frequent equal-sized payments on the total profit of a two-level (vendorbuyer) supply chain system operating under a CS policy.

In this chapter, three payment delay scenarios are studied and their mathematical models developed. The first scenario is when the buyer pays the vendor once it receives an invoice (No delay-in-payment). The second scenario is when the buyer settles its payment by the end of the permissible period (Interest-free period). The last scenario is when the buyer settles its balance after the payment's due date (Interest-charge period).

The application method of the trade credit can be described in the following way. A vendor invoices the buyer for the amount it owes after the latter receives a shipment, and allows the buyer a period of time to settle its payment at no cost. However, an additional cost is incurred if the buyer 
exceeds the permissible delay period. A delay-in-payment, therefore, is the elapsed time between receiving the invoice from the vendor and the payment due date. The invoice details the number of items withdrawn from the consignment inventory (CI) from the date of the last invoice. The buyer is invoiced at intervals and offered a grace period to settle its balance (i.e. 7, 30, 45, or 60 days) at no additional charges (Chapter 5); interest is charged on the unpaid balance once the buyer defers the payment till after its due date.

In order to clearly show the effect of the delay-in-payments when incorporated with the CS agreement, the following straightforward assumptions (in line with the ones used in literature) were considered when developing the models:

1- The buyer's demand is constant.

2- The buyer receives equal instantaneous shipments.

3- The vendor's production rate is constant and greater than the demand rate to avoid shortages.

4- Equal payments at equal time intervals (Chapter 5) and the delay-in-payment is applied for each payment.

5- The vendor's holding cost is divided into two parts; financial and storage. The financial part for items in its inventory is excluded where the one for the items stored in the buyer's warehouse is counted for until the buyer settles its account.

6- The buyer incurs just a storage holding cost. (Table 6-2, next page, illustrates the part of the holding costs that each player incurs)

7- The vendor and the buyer have fixed setup and order costs that are independent of the produced and ordered quantities.

8- Both the vendor and the buyer have a common cycle time. 
Table 6-2. The distribution of the holding cost between the vendor and the buyer

\begin{tabular}{cccc}
\cline { 3 - 3 } & \multicolumn{2}{c}{ Position of raw materials or products } \\
\cline { 3 - 4 } Costs & Vendor & Vendor & Buyer \\
\hline \multirow{3}{*}{ Buyer } & $h_{v, s}$ & $h_{v, f}$ \\
& Buyer & - & $h_{b, s}$ \\
\hline
\end{tabular}

The following notations were used for the input parameters:

\begin{tabular}{|c|c|}
\hline$T$ & cycle time \\
\hline$D$ & demand rate (units/year) \\
\hline$P$ & production rate (units/year) \\
\hline$\gamma$ & number of units (components) needed to produce one item \\
\hline$t$ & time of the invoice \\
\hline$\alpha$ & $\begin{array}{l}\text { fraction of the invoice's time given to the buyer to settle down its payment } \\
\text { (interest-free) }\end{array}$ \\
\hline$\beta$ & $\begin{array}{l}\text { fraction of the invoice's time plus the permissible free period in which the } \\
\text { buyer settles its payment (interest-charge) }\end{array}$ \\
\hline$S_{v}$ & vendor's batch set-up cost (\$/set-up) \\
\hline$I_{v}$ & vendor's investment interest rate $(\% /$ year $)$ \\
\hline$c_{b}$ & vendor's finished product selling price ( $\$ /$ unit) \\
\hline$c_{p}$ & vendor's raw material purchasing cost (\$/unit) \\
\hline$c_{p r}$ & vendor's production cost (\$/unit) \\
\hline$h_{v, f}$ & $\begin{array}{l}\text { vendor's financial-holding cost per item and per time period (\$/unit/year), } \\
\text { equals to } c_{b} \cdot I_{v}\end{array}$ \\
\hline$h_{v, s}$ & vendor's storage-holding cost per item and per time period (\$/unit/year) \\
\hline$O_{b}$ & buyer's ordering cost (\$/order) \\
\hline$h_{b, s}$ & buyer's storage-holding cost per item and per time period ( $\$$ unit/year) \\
\hline$I_{b}$ & buyer's investment interest rate (\%/year) \\
\hline$c_{t}$ & buyer's transaction cost (\$/transaction) \\
\hline & ouyer's finished product selling price $(\$$ \\
\hline
\end{tabular}

The following notations were used for the decision variables:

$n_{i} \quad$ number of shipments (integer) to the buyer for scenario $i$, where $i=1,2$, and 3 (unit-less) 
$m_{i} \quad$ number of payments (integer) in one full cycle for scenario $i$, where $i$ $=1,2$, and 3 ( unit-less)

$q_{i}\left(n_{i}, m_{i}\right)$ quantity size shipped to the buyer for scenario $i$, where $i=1,2$, and 3 (units)

The next section, Section 6.1, is for presenting Scenario 1. Section 6.2 is for Scenario 2. Section 6.3 presents Scenario 3. Section 6.4 is for the solution procedure. Section 6.5 presents and discusses the numerical results and compares them with the classical model. Section 6.6 is for the sensitivity analysis. Section 6.7 represents managerial insights. The chapter closes with Section 6.8, which is for summary and conclusions.

\subsection{Scenario 1: CS without a delay-in-payment}

This scenario studies the effect of the CS agreement when the buyer makes equal-sized payments at equal intervals to the vendor with no delay on the total supply chain profit. Although this scenario is similar to Scheme 1 developed in Chapter 5, calculating the financial-holding (losing opportunity) cost for the vendor is different in this chapter. In Chapter 5, we wanted to show how different payment schemes affect the total profit of a two-level supply chain with a CS agreement. The calculation of the area in Figure 5-1 (Chapter 5) that represents the financial-holding cost of the vendor is an approximation, which is accurately calculated in this chapter. The difference between the two calculations is provided in Appendix 2. To illustrate, it is assumed for this scenario that the area of the financial-holding (represented in grey rectangles in Figure 6-1 next page) accumulates for a period of time $t$, which is the elapsed time between successive invoices. In each buyer's cycle of length $T, m$ equal payments are made to the vendor every $t=T / m$ units of time and $n$ equal shipments of size $q$ each are received. Then, the area shrinks by the amount paid to the vendor at time $t$, the dollar equivalence of $D t$ units, reducing cost and increasing profits. The buyer's revenue comes from selling the items and investing its amount at an annual rate of $I_{b}$ for $t$ units of time. On the other hand, the vendor loses investing its money as it is tied up to inventory in the buyer's warehouse. The behavior of this scenario is shown in Figure 6-1 (next page). 


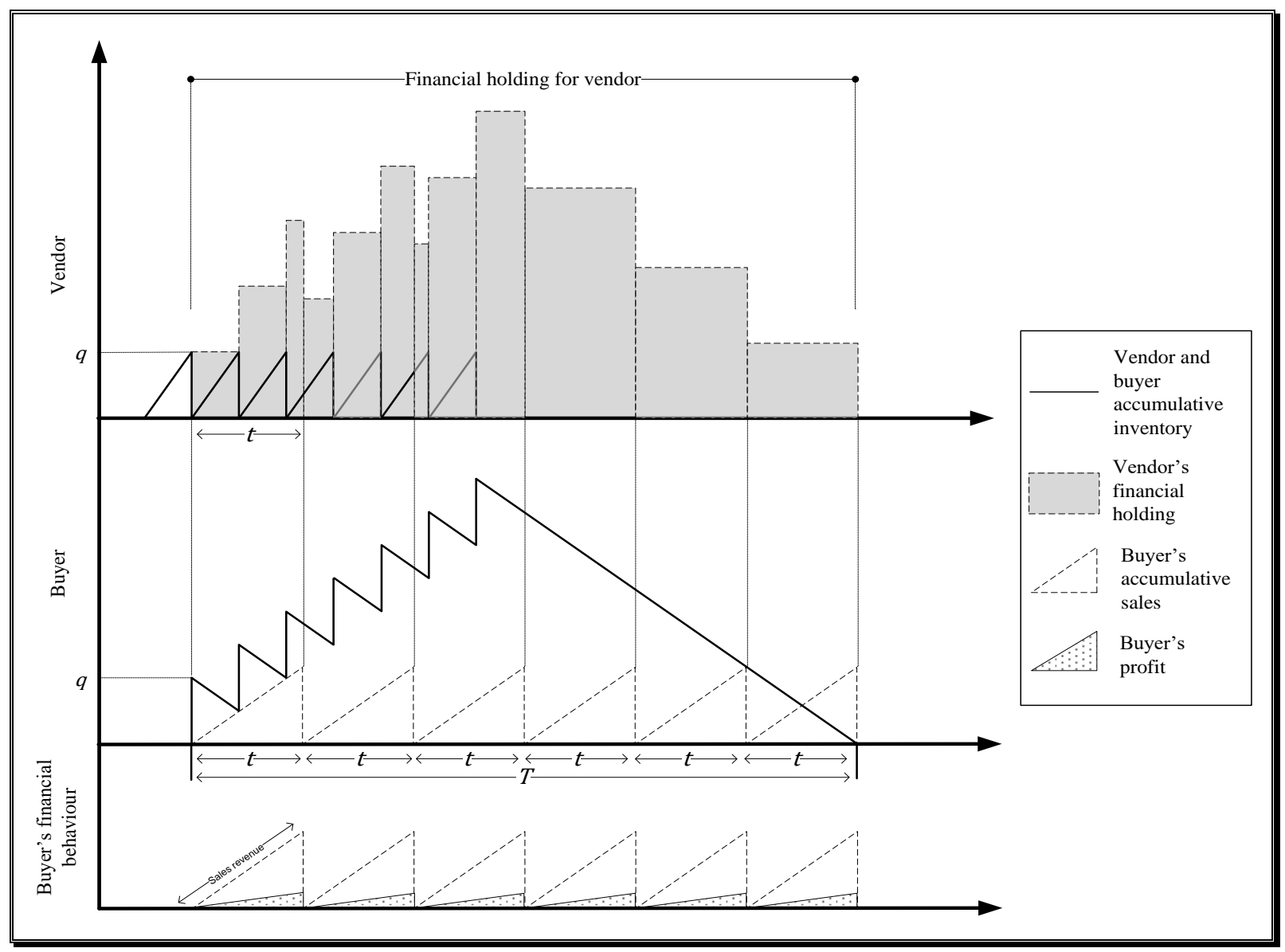

Figure 6-1. Behavior of the consignment stock with no delay-in-payment

Figure 6-1 is divided into three parts $(a, b$, and c). Figure 6-1(a) identifies the behavior of the vendor's storage-holding and financial-holding costs, which are represented by solid line triangles and grey rectangles, respectively. Note that the financial-holding behavior changes as $t$ increases or decreases.

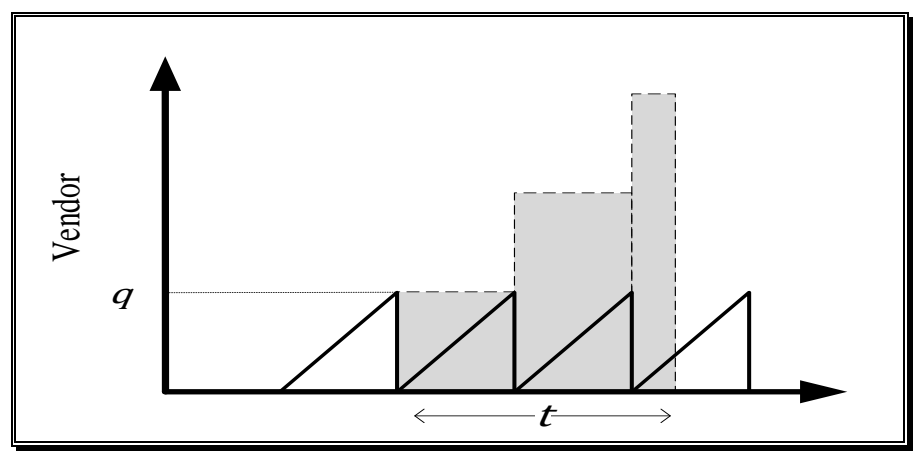

Figure 6-1(a). Vendor's storage and financial-holding behavior within $t$ 
Figure 6-1(b) shows part of the buyer's storage behavior (solid line) and the accumulative sales (dashed triangle) during $t$, which is when the buyer is invoiced for the quantities withdrawn from inventory and makes a payment.

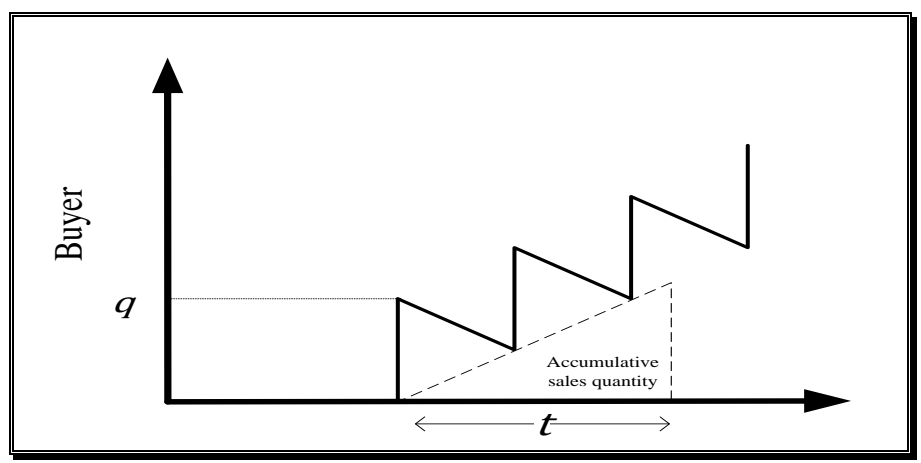

Figure 6-1(b). Buyer's inventory and accumulative sales behavior within $t$

Figure 6-1(c) represents the buyer's financial behavior, which consists of the sales revenue generated from selling items (white dashed triangle), and the profit generated from investing the revenue generated (white dotted triangle) over period $t$.

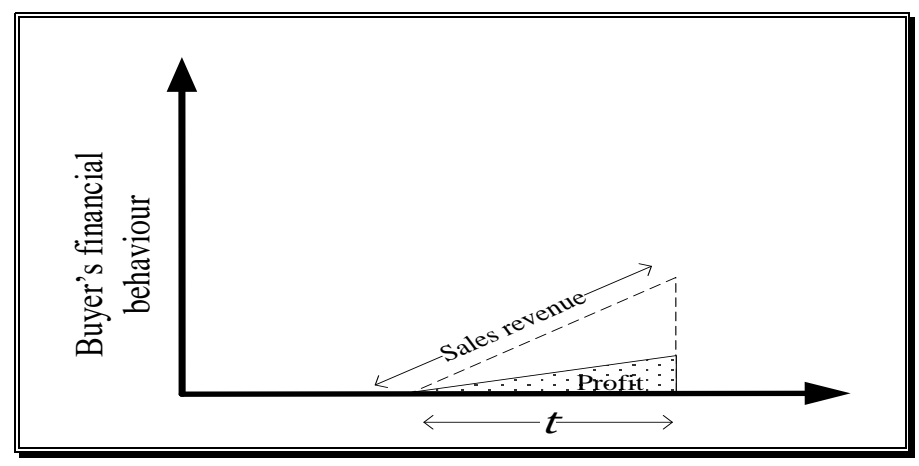

Figure 6-1(c). Buyer's revenue and profit within $t$

The vendor incurs raw material, set-up, production, and storage-holding costs. Other costs considered in Chapter 5, such as the removing and replacing any unsold, unused, or expired items and the audit of inventory costs, are ignored in this work. The vendor incurs a losing opportunity (financial-holding) cost as its money is tied up in inventory at the buyer's side, until the latter is invoiced and pays for the items sold within $t$. This cost is calculated by summing up the areas of the grey rectangles in Figure 6-1 (at the vendor side), and then multiplying the sum by the vendor's unit selling price $c_{b}$ and the vendor's interest rate $I_{v}$ (return on an invested dollar). The vendor's annual financial-holding cost, $C_{v, 1}^{h, f}$, is determined from Figure 6-1 as 


$$
C_{v, 1}^{h, f}=h_{v, f}\left(\frac{\left(m_{1}+1\right) n_{1} q_{1}}{2 m_{1}}-\frac{\left(n_{1}-1\right) q_{1} D}{2 P}\right)
$$

, where $h_{v, f}=c_{b} I_{v}$. The vendor's total annual cost, $T C_{v, 1}$, is the sum of raw material, production, set-up, financial-holding, and storage-holding costs, and is given as

$$
T C_{v, 1}=\left(\gamma c_{p}+c_{p r}\right) D+S_{v} \frac{D}{n_{1} q_{1}}+h_{v, f} \frac{\left(m_{1}+1\right) n_{1} q_{1}}{2 m_{1}}+\left(h_{v, s}-\left(n_{1}-1\right) h_{v, f}\right) \frac{q_{1} D}{2 P}
$$

The buyer's total annual cost, $T C_{b, 1}$, is the sum of purchasing, ordering, transaction, and storageholding costs, and is written as

$$
T C_{b, 1}=c_{b} D+\left(n_{1} O_{b}+m_{1} c_{t}\right) \frac{D}{n_{1} q_{1}}+h_{b, s}\left(\frac{n_{1} q_{1}}{2}-\left(n_{1}-1\right) \frac{q_{1} D}{2 P}\right)
$$

The vendor's annual revenue is $T R_{v, 1}=c_{b} D$. The buyer's annual revenue, $T R_{b, 1}$, is generated from selling items and from investing the sales revenue (second part of Eq. (6-4)), and is given as

$$
T R_{b, 1}=c_{c}\left(D+I_{b} \frac{n_{1} q_{1}}{2 m_{1}}\right)
$$

The total supply chain profit for Scenario $i=1,2$, and 3, is $T P_{s, i}=T R_{v, i}+T R_{b, i}-T C_{v, i}-$ $T C_{b, i}$, where $T P_{s, i}$ is a function of $n_{i}, m_{i}$, and $q_{i}$. The total profit function for Scenario 1 is written as

$$
\begin{aligned}
& T P_{s, 1}\left(q_{1}, n_{1}, m_{1}\right)=\left(c_{c}-\gamma c_{p}-c_{p r}\right) D-\left[\left(S_{v}+n_{1} O_{b}+m_{1} c_{t}\right) \frac{D}{n_{1} q_{1}}+\frac{\left(h_{v, f}+h_{b, s}\right) n_{1} q_{1}}{2}(1-\right. \\
& \left.\left.\frac{D}{P}\right)+\left(h_{v, f}-c_{c} I_{b}\right) \frac{n_{1} q_{1}}{2 m_{1}}+\left(h_{v, s}+h_{v, f}+h_{b, s}\right) \frac{q_{1} D}{2 P}\right]
\end{aligned}
$$

The concavity of the profit function, Eq. (6-5), can be demonstrated without the use of derivatives and the necessary sufficient conditions for the second-order. This approach does not use classical differential calculus (Grubbström and Erdem, 1999; Zanoni and Grubbstrom, 2004), and found to be robust. Thus, the optimal quantity that maximizes Eq. (6-5) is given as 


$$
\begin{aligned}
& q_{1}\left(n_{1}, m_{1}\right) \\
& =\sqrt{\frac{2\left(S_{v}+n_{1} O_{b}+m_{1} c_{t}\right) D}{n_{1}^{2}\left[\left(h_{v, f}+h_{b, s}\right)\left(1-\frac{D}{P}\right)+\left(h_{v, f}-c_{c} I_{b}\right) \frac{1}{m_{1}}+\left(h_{v, s}+h_{v, f}+h_{b, s}\right) \frac{D}{n_{1} P}\right]}}
\end{aligned}
$$

The denominator of Eq. (6-6) is always positive (see Appendix 3). Substituting Eq. (6-6) in Eq. (6-5) reduces it to a function of two variables, so it appears as $T P_{s, 1}\left(q_{1}\left(n_{1}, m_{1}\right), n_{1}, m_{1}\right)=$ $T P_{s, 1}\left(n_{1}, m_{1}\right)$. There is ample evidence of this practice in the literature. Using the suggested approach, the optimum real values of the $n_{1}^{*}$ and $m_{1}^{*}$, which are independent from one another, are computed as seen in Eqs. (10-10) and (10-12), respectively (Appendix 4).

So, the optimum values of Eqs. (6-6) and (6-5) are computed using the values of the $n_{1}^{*}$ and $m_{1}^{*}$ as $q_{1}^{*}\left(n_{1}^{*}, m_{1}^{*}\right)$ and $T P_{s, 1}^{*}\left(n_{1}^{*}, m_{1}^{*}\right)=T P_{s, 1}^{*}\left(q_{1}^{*}\left(n_{1}^{*}, m_{1}^{*}\right), n_{1}^{*}, m_{1}^{*}\right)$, respectively. Note that the best value of the profit function for Scenario (model) $i=1,2$, and 3 is determined as $T P_{s, i}^{*}\left(n_{i}^{*}, m_{i}^{*}\right)=\max \left\{T P_{s, i}\left(\left\lfloor n_{i}^{*}\right\rfloor,\left\lfloor m_{i}^{*}\right\rfloor\right), T P_{s, i}\left(\left\lfloor n_{i}^{*}\right\rfloor,\left\lceil m_{i}^{*}\right\rceil\right), T P_{s, 1}\left(\left\lceil n_{i}^{*}\right\rceil,\left\lfloor m_{i}^{*}\right\rfloor\right), T P_{s, i}\left(\left\lceil n_{i}^{*}\right\rceil,\left\lceil m_{i}^{*}\right\rceil\right)\right\} \quad$, and accordingly $q_{i}^{*}\left(n_{i}^{*}, m_{i}^{*}\right)$. The mathematics for Scenarios (models) 2 and 3 is shown in Sections 6.2 and 6.3 , respectively.

\subsection{Scenario 2: CS with a delay-in-payment (Interest-free)}

This scenario assumes that a delay-in-payment is offered by the vendor and the buyer pays the vendor by the end of the permissible period, $\tau$, which is equal to $t=T / m$ (the time of the invoice) plus $\alpha t$ (interest-free delay period), where $\alpha>0$. The buyer invests the revenue generated from selling the product for a period of $\tau=t+\alpha t$, which is an opportunity loss period for the vendor, at an interest rate $I_{b}$. Figure 6-2 (next page) illustrates the behavior of gains and losses for both players.

Figure 6-2 is similar to Figure 6-1 with only one difference, which is the inclusion of the permissible delay period $\alpha t$ (highlighted in black horizontal lines). Figure 6-2 can be divided into three parts $(\mathrm{a}, \mathrm{b}$, and $\mathrm{c})$ for better illustration. 


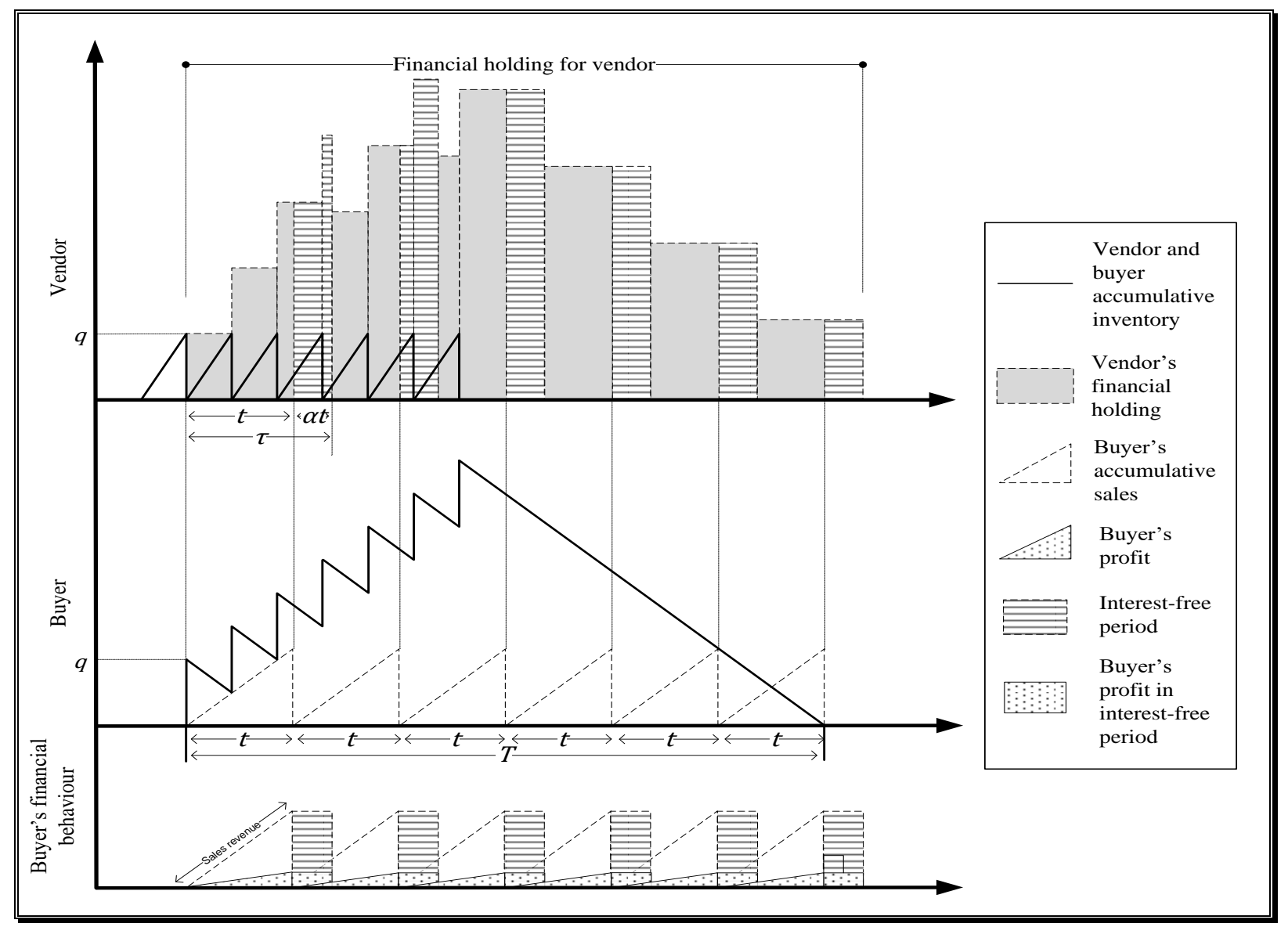

Figure 6-2. Behavior of the consignment stock with delay-in-payment (Interest-free)

Figure 6-2(a) represents the storage-holding and the financial-holding behavior of the vendor. Comparing Figure 6-2(a) with Figure 6-1(a), the reader can realize that the only difference is the behavior of the financial-holding area, which continues to accumulate beyond the invoice period $t$ (the end of the grey area) to $\tau$ (the end of the black horizontal lines).

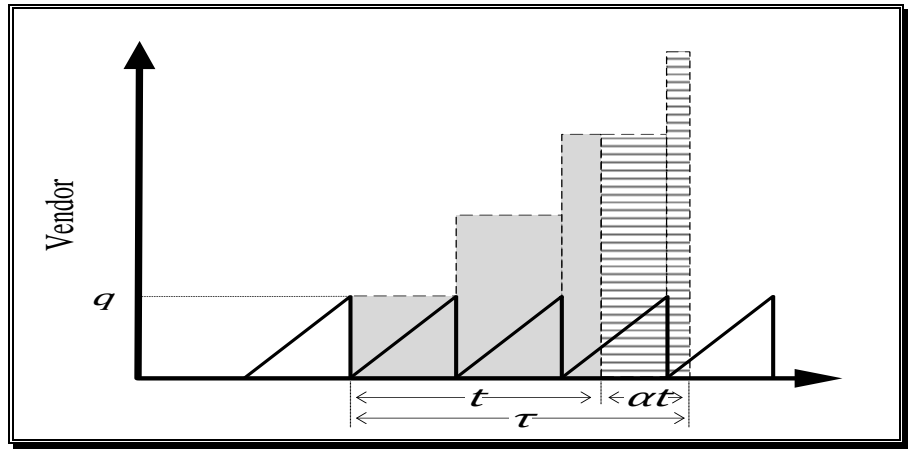

Figure 6-2(a). Vendor's storage and financial-holding behavior within $\tau$ 
Figure 6-2(b) shows part of the storage (solid line), and the cumulative sales (dashed triangle) for the buyer within $\tau$. Sales during $\alpha t$ are accounted for in the next invoice.

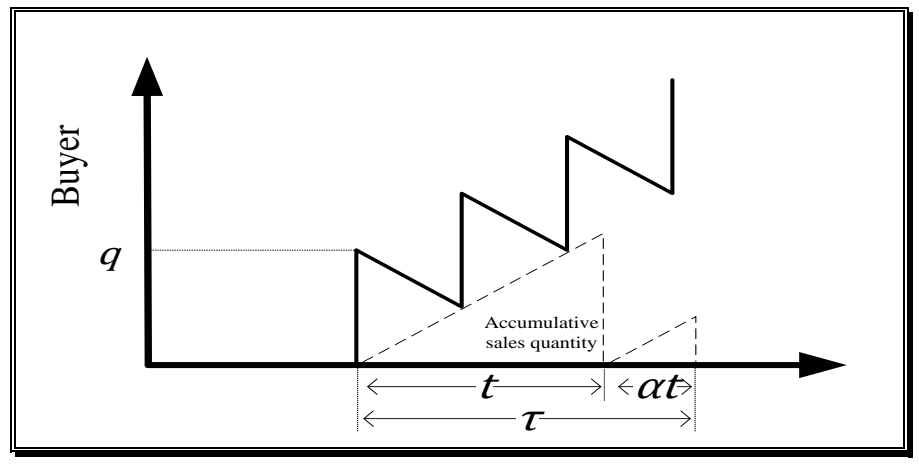

Figure 6-2(b). Buyer's inventory and accumulative sales behavior within $\tau$

Figure 6-2(c) shows the buyer's revenue from sales during $t$ (dashed triangle) and from investment (white dotted triangle), where the buyer invests for a longer period, $\tau=t+\alpha t$, thus generating more revenue. However, the additional sales revenue is accounted for in the next cycle. The profit generated in $\alpha t$ (represented in the solid white dotted rectangle) is equal to $c_{c} \alpha D t^{2} I_{b}$.

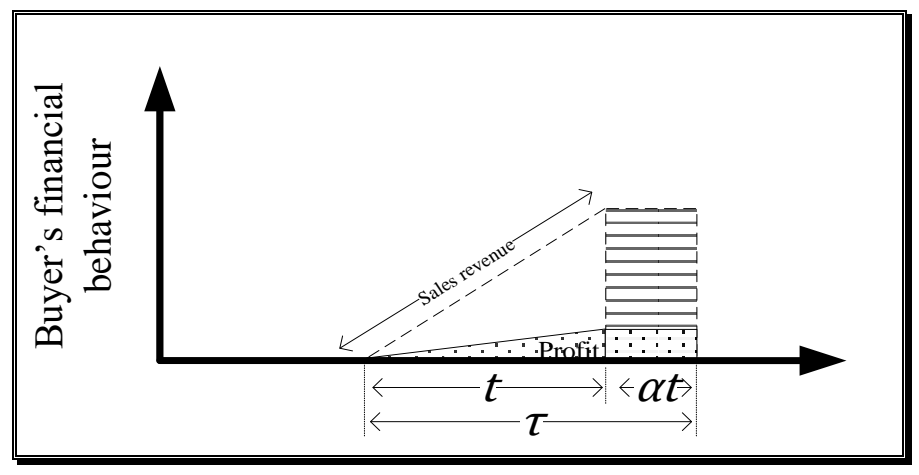

Figure 6-2(c). Buyer's revenue and profit within $\tau$

Figure 6-2 is used to calculate all costs and revenues for the vendor and the buyer. The total cost for the vendor is calculated in a similar manner to that of Scenario 1. The vendor's opportunity loss for this scenario extends for a longer period, $\tau>t$, and it is written as

$$
C_{v, 2}^{h, f}=h_{v, f}\left(\frac{\left(m_{2}+1+2 \alpha\right) n_{2} q_{2}}{2 m_{2}}-\frac{\left(n_{2}-1\right) q_{2} D}{2 P}\right)
$$

As the buyer is allowed to settle its balance by time $\tau=t+\alpha t$, the vendor's total annual cost is written as 


$$
\begin{aligned}
T C_{v, 2}=\left(\gamma c_{p}\right. & \left.+c_{p r}\right) D+S_{v} \frac{D}{n_{2} q_{2}}+h_{v, f} \frac{\left(m_{2}+1+2 \alpha\right) n_{2} q_{2}}{2 m_{2}} \\
& +\left(h_{v, s}-\left(n_{2}-1\right) h_{v, f}\right) \frac{q_{2} D}{2 P}
\end{aligned}
$$

The buyer's total cost for Scenario 2 is calculated using Eq. (6-3). The vendor's annual revenue is the same as for Scenario 1, i.e. $T R_{v, 1}=c_{b} D$. The buyer's annual revenue of $T R_{b, 2}$ from sales and investment is equal to

$$
T R_{b, 2}=c_{c}\left(D+I_{b} \frac{(2 \alpha+1) n_{2} q_{2}}{2 m_{2}}\right)
$$

The total annual supply chain profit of the system is determined as before and is written as

$$
\begin{aligned}
T P_{s, 2}\left(q_{2}, n_{2}, m_{2}\right)= & \left(c_{c}-\gamma c_{p}-c_{p r}\right) D-\left[\left(S_{v}+n_{2} O_{b}+m_{2} c_{t}\right) \frac{D}{n_{2} q_{2}}+\right. \\
& \frac{\left(h_{v, f}+h_{b, s}\right) n_{2} q_{2}}{2}\left(1-\frac{D}{P}\right)+\left(h_{v, f}-c_{c} I_{b}\right) \frac{(2 \alpha+1) n_{2} q_{2}}{2 m_{2}}+\left(h_{v, s}+h_{v, f}+6-10\right. \\
& \left.\left.h_{b, s}\right) \frac{q_{2} D}{2 P}\right]
\end{aligned}
$$

The concavity of the profit function, Eq. (6-10), is found using the approach of Grubbström and Erdem (1999) as explained following Eq. (6-5) (see Appendix 4). Thus, the optimal quantity that maximizes Eq. (6-10) is given as

$$
q_{2}\left(n_{2}, m_{2}\right)=\sqrt{\frac{2\left(S_{v}+n_{2} o_{b}+m_{2} c_{t}\right) D}{n_{2}^{2}\left[\left(h_{v, f}+h_{b, s}\right)\left(1-\frac{D}{P}\right)+\left(h_{v, f}-c_{c} I_{b}\right) \frac{(2 \alpha+1)}{m_{2}}+\left(h_{v, s}+h_{v, f}+h_{b, s}\right) \frac{D}{n_{2} P}\right]}}
$$

The denominator of Eq. (6-11) is almost identical to that of Eq. (6-6) and can be shown to be always positive as explained in Appendix 4. The closed form expressions for $n_{2}^{*}$ and $m_{2}^{*}$, which are independent from one another, are computed from Eqs. (10-15) and (10-16), respectively, using the approach of Grubbström and Erdem (1999) in Appendix 4 and as explained following Eq. (6-6).

\subsection{Scenario 3: CS with a delay-in-payment (Interest-charge)}

In the last scenario the vendor offers the buyer an interest-free period $\alpha t$ to settle its balance of the invoice received at time $t$. The buyer may defer a payment by $\delta=\tau+\beta \tau$, where $\beta>0$, but the 
unpaid balance subjects to interest charges. In this scenario, the buyer has a longer period, to invest its revenue. Figure 6-3 illustrates the behavior of gains and losses for both players.

Figure 6-3 is similar to Figure 6-1 with only two differences, which are the period allowed for the buyer to settle its balance at no cost (highlighted in black horizontal lines), and the time where the buyer settles it after the allowed period at an additional cost (highlighted in black vertical lines). This figure is divided for three parts $(\mathrm{a}, \mathrm{b}$, and $\mathrm{c})$ for better illustration.

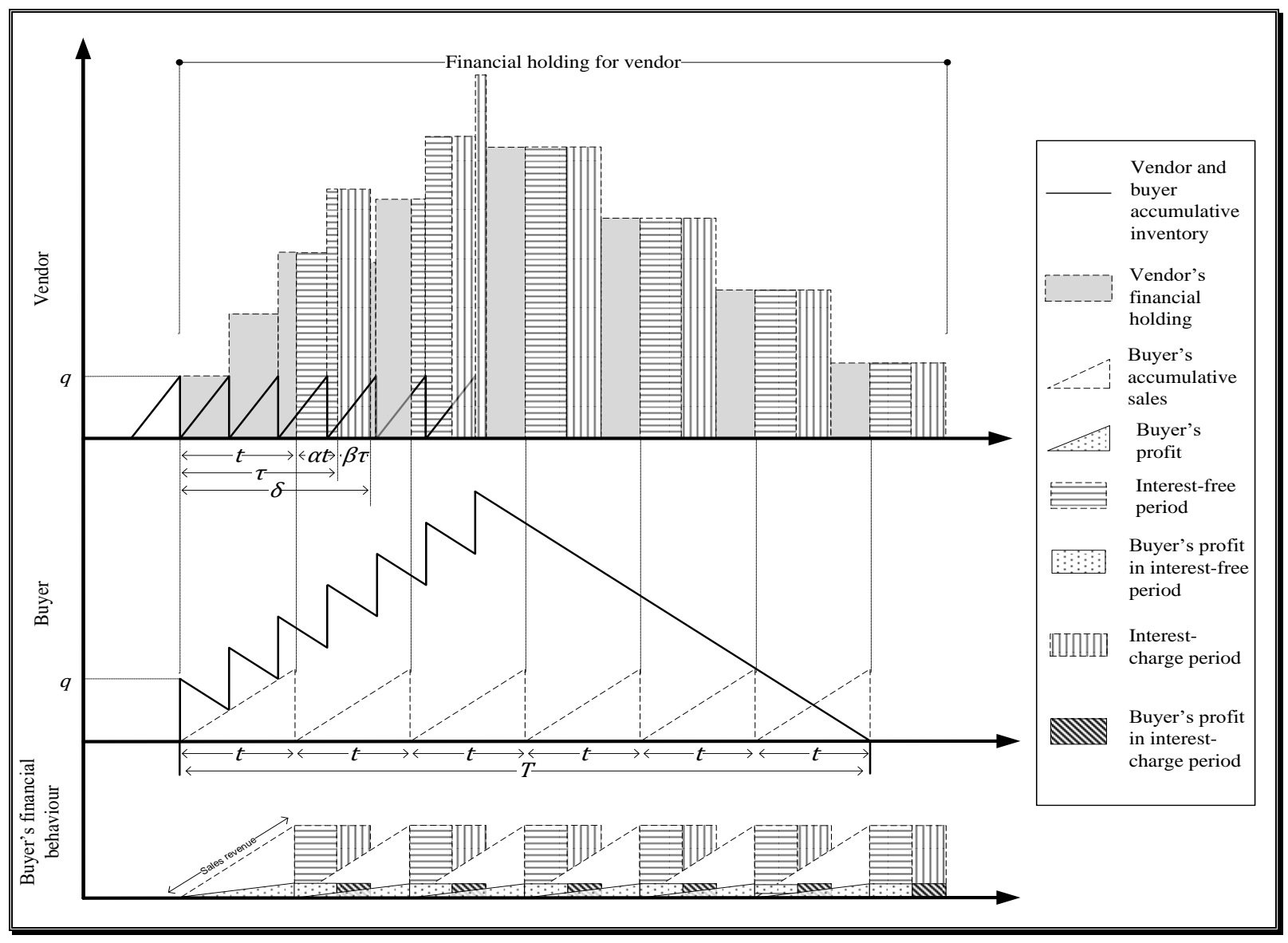

Figure 6-3. Behavior of the consignment stock with delay-in-payment (interest-charge)

Figure 6-3(a), next page, shows the behavior of the storage-holding and the financial-holding of the vendor. Comparing Figure 6-3(a) with Figure 6-2(a), the reader notices that the behavior of the financial-holding area of the vendor still accumulates until the buyer settles its balance by time $\delta$ (the end of the area shaded in black vertical lines) after being invoiced at time $t$ (the end of the grey area), with the offer of making a payment at time $\tau$ at no additional charges (the end of the area shaded in black horizontal lines). 


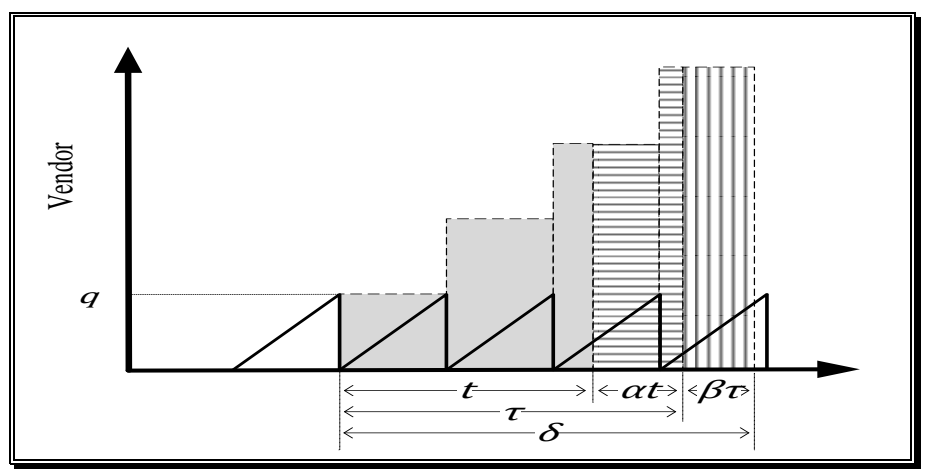

Figure 6-3(a). Vendor's storage and financial-holding behavior within $\delta$

The behavior of the buyer's inventory, shown in Figure 6-3(b), remains unchanged. Although the buyer sells more quantity between the times it receives the invoice, $t$, and the time it makes the payment, $\delta$, the sales generated during $\alpha t+\beta \tau$ are added to the next invoice.

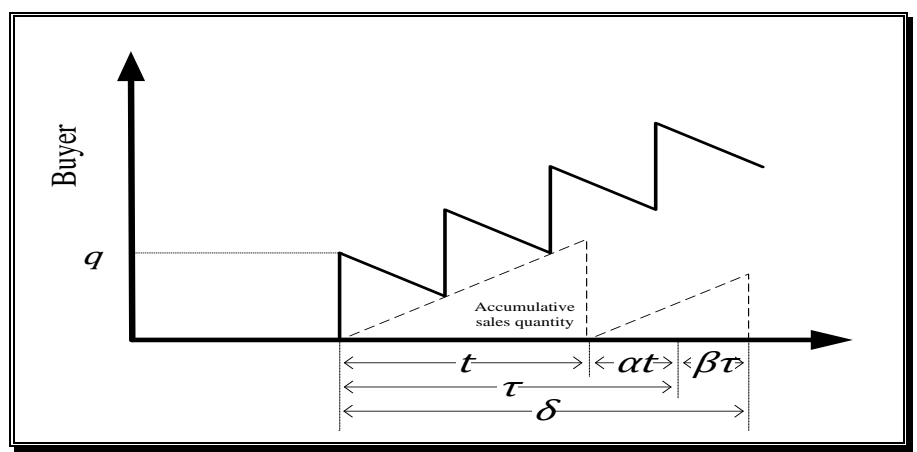

Figure 6-3(b). Buyer's inventory and accumulative sales behavior within $\delta$

Figure 6-3(c) shows the revenue that the buyer makes within $t$ (dashed triangle) and the profit from investing that revenue (white dotted triangle), which is $c_{c} D t$ for $\alpha t+\beta \tau$. The net profit that the buyer makes during $\alpha t$ is more than what it makes during $\beta \tau$ because of the interest charged. The revenue generated from selling items after the invoice time $t$ appears in the next invoice.

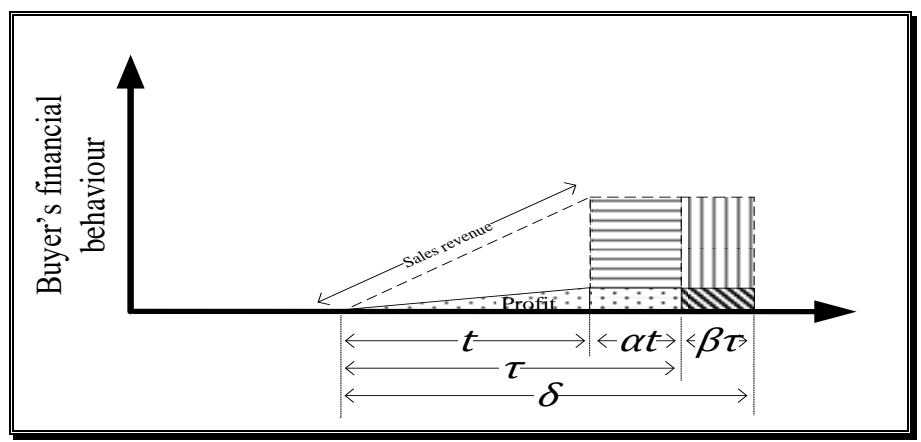

Figure 6-3(c). Buyer's revenue and profit within $\delta$ 
Therefore, the sum of the profits generated in $\alpha t$ (represented in the white dotted rectangle), and in $\beta \tau$ (represented in the diagonal lines shaded rectangle) period of times is equal to $c_{c} D t I_{b}(\alpha t+\beta \tau)=c_{c} D t^{2} I_{b}(\alpha+\beta(1+\alpha))$.

When the buyer settles its payment after the invoice date and benefits from the permissible delay period, the interest-free and the non-interest free, the financial-holding cost per cycle is calculated by summing up the areas of the rectangles shaded in grey, and areas under the horizontal and vertical lines in Figure 6-3 (at the vendor side). The sum of the areas is multiplied by $c_{b} I_{v}$, which represents an opportunity loss to the vendor, and is written as

$$
C_{v, 3}^{h, f}=h_{v, f}\left(\frac{\left(m_{3}+1+2 \alpha+2 \beta(1+\alpha)\right) n_{3} q_{3}}{2 m_{3}}-\frac{\left(n_{3}-1\right) q_{3} D}{2 P}\right)
$$

The vendor's total annual cost is given as

$$
\begin{aligned}
T C_{v, 3}=\left(\gamma c_{p}\right. & \left.+c_{p r}\right) D+S_{v} \frac{D}{n_{3} q_{3}}+h_{v, f} \frac{\left(m_{3}+1+2 \alpha+2 \beta(1+\alpha)\right) n_{3} q_{3}}{2 m_{3}} \\
& +\left(h_{v, s}-\left(n_{3}-1\right) h_{v, f}\right) \frac{q_{3} D}{2 P}
\end{aligned}
$$

The buyer's total cost is written in a similar manner to the previous scenarios except for the additional charges caused by deferring the payment by $\beta \tau$, which incurs a cost per cycle of $(\delta-\tau) c_{b} I_{v} D T$. The buyer's total annual cost is then written as

$$
\begin{aligned}
T C_{b, 3}=c_{b} D+ & \left(n_{3} O_{b}+m_{3} c_{t}\right) \frac{D}{n_{3} q_{3}}+h_{b, s}\left(\frac{n_{3} q_{3}}{2}-\left(n_{3}-1\right) \frac{q_{3} D}{2 P}\right) \\
& +c_{b} I_{v} \frac{\beta(1+\alpha) n_{3} q_{3}}{m_{3}}
\end{aligned}
$$

The vendor makes revenue from selling the items to the buyer and the interest charged on unsettled balances, and is written as

$$
T R_{v, 3}=c_{b}\left(D+I_{v} \frac{\beta(1+\alpha) n_{3} q_{3}}{m_{3}}\right)
$$


Similarly, the buyer's annual revenue is generated from sales and investment and is written as

$$
T R_{b, 3}=c_{c}\left(D+I_{b} \frac{(2 \alpha+1+2 \beta(1+\alpha)) n_{3} q_{3}}{2 m_{3}}\right)
$$

The total annual supply chain profit is determined in a similar manner as before and is written as

$$
\begin{aligned}
T P_{s, 3}\left(q_{3}, n_{3}, m_{3}\right)= & \left(c_{c}-\gamma c_{p}-c_{p r}\right) D-\left[\left(S_{v}+n_{3} O_{b}+m_{3} c_{t}\right) \frac{D}{n_{3} q_{3}}+\right. \\
& \frac{\left(h_{v, f}+h_{b, s}\right) n_{3} q_{3}}{2}\left(1-\frac{D}{P}\right)+\left(h_{v, f}-c_{c} I_{b}\right) \frac{(2 \alpha+1+2 \beta(1+\alpha)) n_{3} q_{3}}{2 m_{3}}+\left(h_{v, f}+6-17\right. \\
& \left.\left.h_{b, s}+h_{v, s}\right) \frac{q_{3} D}{2 P}\right]
\end{aligned}
$$

The concavity of the profit function, Eq. (6-17), is found using the approach of Grubbström and Erdem, (1999) as explained following Eq. (6-5) (see Appendix 4). This approach does not use classical differential calculus. Thus, the optimal quantity that maximizes Eq. (6-17) is given as

$$
q_{3}\left(n_{3}, m_{3}\right)=\sqrt{\frac{2\left(S_{v}+n_{3} O_{b}+m_{3} c_{t}\right) D}{n_{3}^{2}\left[\left(h_{v, f}+h_{b, s}\right)\left(1-\frac{D}{P}\right)+\left(h_{v, f}-c_{c} I_{b}\right) \frac{(2 \alpha+1+2 \beta(1+\alpha))}{m_{3}}+\left(h_{v, f}+h_{b, s}+h_{v, s}\right) \frac{D}{n_{3} P}\right]}}
$$

The denominator of Eq. (6-18) is almost identical to that of Eq. (6-6) and can be shown to be positive as illustrated in Appendix 3. The closed form expressions for $n_{3}^{*}$ and $m_{3}^{*}$, which are independent from one another, are computed from Eqs. (10-19) and (10-20), respectively, using the approach of Grubbström and Erdem (1999) in Appendix 4 and as explained following Eq. (6-6).

\subsection{Solution Procedure}

This section provides a solution procedure to find the optimal solution (number of shipments $n_{i}^{*}$, number of payments $m_{i}^{*}$, and quantity $\left.q_{i}^{*}\left(n_{i}^{*}, m_{i}^{*}\right)\right)$ for each scenario developed in the previous sections using the nested loop search, similar to the one described in Jaber and Goyal (2008) and Chapter 5. The procedure can be summarized as follow.

1- Input the given parameters; for the vendor $\left(P, S_{v}, I_{v}, \gamma, h_{v, f}, h_{v, s}, \beta, \alpha, c_{p}, c_{p r}, c_{b}\right)$ and for the buyer $\left(D, O_{b}, h_{b, s}, I_{b}, c_{b}, c_{c}, c_{t}, \beta\right)$. 
2- Start searching for the optimal number of shipments $\left(n_{i}^{*}\right)$ and payments $\left(m_{i}^{*}\right)$, that give you the optimal quantity $\left(q_{i}^{*}\right)$, where $i=1,2$ and 3 and represents the developed scenarios, by following these steps:

a. Iteration 1:

i. Start by setting $n_{i}=1$ and $m_{i}=1$ and then,

ii. Compute $q_{i}\left(n_{i}, m_{i}\right)$ using Eqs. (6-6), (6-11), and (6-18) for Scenario 1, 2 and 3 respectively.

iii. Use the values in Steps $a-i$ and $a-i i$, find the value of $T P_{s, i}\left(n_{i}, m_{i}, q_{i}\left(n_{i}, m_{i}\right)\right)$ using Eqs. (6-5), (6-10), and (6-17).

iv. Store the value from Step a-iii to be Value 1, i.e., $T P_{s, i}\left(1,1, q_{i}(1,1)\right)$ for later comparison.

v. Increase the value of $m_{i}$ by 1 (i.e. $m_{i}=2$ ), and repeat Steps a-ii and a-iii. Store the value as Value 2; i.e. $T P_{s, i}\left(1,2, q_{i}(1,2)\right)$.

vi. Compare Value 1 with Value 2. If Value $1>$ Value 2, then Value 1 is optimal and the corresponding values of $n_{i}, m_{i}$ and $q_{i}\left(n_{i}, m_{i}\right)$ are optimal. Otherwise, set Value 2 as Value 1 , increase $m_{i}$ by 1 (i.e. $m_{i}=3$ ), and repeat the Steps (a-ii and a-iii) and store this value as Value 2.

vii. Continue until you reach an optimal value from this iteration where $T P_{s, i}\left(1, m_{i}^{*}, q_{i}\left(n_{i}, m_{i}^{*}\right)\right)>T P_{s, i}\left(1, m_{i}^{*}+1, q_{i}\left(1, m_{i}^{*}+1\right)\right)$ and $T P_{s, i}\left(1, m_{i}^{*}, q_{i}\left(n_{i}, m_{i}^{*}\right)\right)>T P_{s, i}\left(1, m_{i}^{*}-1, q_{i}\left(1, m_{i}^{*}-1\right)\right)$. Then, store this value as Value 1 for later comparison, and go to the next iteration.

b. Iteration 2:

i. Increase $n_{i}$ by 1 (i.e. $n_{i}=2$ ) and follow the same steps in Iteration 1 by finding $q_{i}\left(n_{i}, m_{i}\right)$ and $T P_{s, i}\left(n_{i}, m_{i}, q_{i}\left(n_{i}, m_{i}\right)\right)$. Store this value as Value 2. Compare this value with Value 1 in Iteration 1 . If Value $1>$ Value 2, then stop as the optimal solution is reached. Otherwise, store Value 2 as optimal 
with its values of $n_{i}, m_{i}$ and $q_{i}\left(n_{i}, m_{i}\right)$ and repeat the same steps in Iteration 1 until you find the optimal solution for this iteration in which

$$
\begin{gathered}
T P_{s, i}\left(2, m_{i}^{*}, q_{i}\left(2, m_{i}^{*}\right)\right)>T P_{s, i}\left(2, m_{i}^{*}+1, q_{i}\left(2, m_{i}^{*}+1\right)\right) \text { and } \\
T P_{s, i}\left(2, m_{i}^{*}, q_{i}\left(2, m_{i}^{*}\right)\right)>T P_{s, i}\left(2, m_{i}^{*}-1, q_{i}\left(2, m_{i}^{*}-1\right)\right) .
\end{gathered}
$$

Repeat Iterations 1 and 2 until no farther improvement is observed. Then, stop and the values of $\left(n_{i}, m_{i}, q_{i}\left(n_{i}, m_{i}\right)\right)$ that give the highest profit are optimal $\left(n_{i}^{*}, m_{i}^{*}, q_{i}^{*}\left(n_{i}^{*}, m_{i}^{*}\right)\right)$, where $n_{i}^{*} \geq 1$ and $m_{i}^{*} \geq 1$.

c. Iteration 3:

i. Plug the optimal values found in the previous iterations in Eqs. (6-2), (6-8) and (6-13) to find the optimal total cost for the vendor, $T C_{v, i}$, where $i$ represents scenario 1, 2, and 3, respectively, and in Eq. (6-3) for the buyer's total cost, $T C_{b, 1}$ and $T C_{b, 2}$ and Eq. (6-14) for $T C_{b, 3}$.

ii. Calculate $T R_{v, i}$ for scenario $i=1,2$ and 3 using the equation $c_{b} D$ for $T R_{v, 1}=T R_{v, 2}$ and Eq. (6-15) for $T R_{v, 3}$, and Eqs. (6-4), (6-9) and (6-16) for $T R_{b, i}$, where $i$ represents Scenario 1, 2 and 3, respectively, using $\left(n_{i}^{*}, m_{i}^{*}, q_{i}^{*}\left(n_{i}^{*}, m_{i}^{*}\right)\right)$ and the values of the parameters.

Finally, calculate the total profit for each player, $\left(T P_{v, i}\right)$ and $\left(T P_{b, i}\right)$ by subtracting the total cost for the player, found in Step c-i, from its total revenue, found in Step c-ii.

\subsection{Numerical Example}

\subsubsection{Comparison between the three scenarios of the CS}

In this section, a numerical example is solved to compare the three scenarios that were developed in Sections 6.1, 6.2 and 6.3. In addition, the numerical example illustrates the behavior of the model for the three scenarios and investigates if a CS policy is better with or without a delay-inpayment. The optimal solution of $n_{i}^{*}$ and $m_{i}^{*}$ for each scenario can be found using the closed form expressions developed in Appendix 4 or the steps of the solution procedure described in Jaber and Goyal (2008). 
The values of the input parameters used in this example are adopted from the literature, where: $P=3,200, D=1,000, S_{v}=400, O_{b}=25, h_{v, s}=4, h_{b, s}=5, \gamma=1, I_{v}=0.1, I_{b}=0.2, c_{p}=$ $3, c_{p r}=1, c_{b}=5.4, c_{c}=7.29$, and $c_{t}=0.5$. Other values were estimated within reason; for instance $h_{v, f}=I_{v} c_{b}=0.54, \alpha=0.2$, and $\beta=0.4$.

Table 6-3, Table 6-4 and Table 6-5 (next page) illustrate the search for the optimal solution for Scenarios 1, 2 and 3, respectively, using the steps developed in Section 6.4, and the optimal solutions were highlighted in bold.

Table 6-3. Search for Scenario 1 optimal solution

\begin{tabular}{ccccccc}
\hline$n_{1}$ & $m_{1}$ & $q_{1}\left(n_{1}, m_{1}\right)$ & $T P_{s, 1}\left(n_{1}, m_{1}, q_{1}\left(n_{1}, m_{1}\right)\right)$ & $\mathrm{V} 1$ & $\mathrm{~V} 2$ & $\begin{array}{c}\text { Optimal } \\
T P_{s, 1}\left(n_{1}, m_{1}, q_{1}\left(n_{1}, m_{1}\right)\right)\end{array}$ \\
\hline 1 & 1 & 381 & $1,054.6$ & & & \\
1 & 2 & 367 & 967.5 & $1,054.6>967.5$ & $1,054.6$ \\
\hline 2 & 1 & 227 & $1,303.1$ & $1,054.6$ & $<1,303.1$ & $1,303.1$ \\
2 & 2 & 216 & $1,200.5$ & $1,303.1>1,200.5$ & $1,303.1$ \\
\hline 3 & 1 & 165 & $1,368.0$ & $1,303.1<1,368.0$ & $1,368.0$ \\
3 & 2 & 156 & $1,256.5$ & $1,368.0>1,256.5$ & $1,368.0$ \\
\hline $\mathbf{4}$ & $\mathbf{1}$ & $\mathbf{1 3 1}$ & $\mathbf{1 , 3 8 2 . 2}$ & $1,368.0$ & $<1,382.2$ & $1,382.2$ \\
4 & 2 & 124 & $1,264.4$ & $1,382.2>1,264.4$ & $1,382.2$ \\
\hline 5 & 1 & 110 & $1,375.6$ & $1,382.2>1,375.6$ & $\mathbf{1 , 3 8 2 . 2}$ \\
\hline
\end{tabular}

(V1: Value 1, V2: Value 2, TP: total profit; subscript $s=$ system, $1=$ Scenario 1)

Table 6-4. Search for Scenario 2 optimal solution

\begin{tabular}{ccccccc}
\hline$n_{2}$ & $m_{2}$ & $q_{2}\left(n_{2}, m_{2}\right)$ & $T P_{s, 2}\left(n_{2}, m_{2}, q_{2}\left(n_{2}, m_{2}\right)\right)$ & $\mathrm{V} 1$ & $\mathrm{~V} 2$ & $\begin{array}{c}\text { Optimal } \\
T P_{s, 2}\left(n_{2}, m_{2}, q_{2}\left(n_{2}, m_{2}\right)\right)\end{array}$ \\
\hline 1 & 1 & 393 & $1,125.6$ & & & \\
1 & 2 & 372 & $1,001.4$ & $1,125.6>1,001.4$ & $1,125.6$ \\
\hline 2 & 1 & 237 & $1,388.2$ & $1,125.6<1,388.2$ & $1,388.2$ \\
2 & 2 & 220 & $1,240.5$ & $1,388.2>1,240.5$ & $1,388.2$ \\
\hline 3 & 1 & 173 & $1,461.1$ & $1,388.2<1,461.1$ & $1,461.1$ \\
3 & 2 & 159 & $1,300.0$ & $1,461.1>1,300.0$ & $1,461.1$ \\
\hline $\mathbf{4}$ & $\mathbf{1}$ & $\mathbf{1 3 8}$ & $\mathbf{1 , 4 8 1 . 1}$ & $1,461.1<1,481.1$ & $1,481.1$ \\
4 & 2 & 127 & $1,310.3$ & $1,481.1>1,310.3$ & $1,481.1$ \\
\hline 5 & 1 & 116 & $1,479.2$ & $1,481.1>1,479.2$ & $\mathbf{1 , 4 8 1 . 1}$ \\
\hline
\end{tabular}

(V1: Value 1, V2: Value 2, TP: total profit; subscript $\mathrm{s}=$ system, $2=$ Scenario 2 ) 
Table 6-5. Search for Scenario 3 optimal solution

\begin{tabular}{|c|c|c|c|c|c|c|c|}
\hline$n_{3}$ & $m_{3}$ & $q_{3}\left(n_{3}, m_{3}\right)$ & $T P_{s, 3}\left(n_{3}, m_{3}, q_{3}\left(n_{3}, m_{3}\right)\right)$ & V1 & & V2 & $\begin{array}{c}\text { Optimal } \\
T P_{s, 3}\left(n_{3}, m_{3}, q_{3}\left(n_{3}, m_{3}\right)\right)\end{array}$ \\
\hline 1 & 1 & 429 & $1,306.4$ & & & & \\
\hline 1 & 2 & 386 & $1,085.0$ & $1,306.4$ & $>$ & $1,085.0$ & $1,306.4$ \\
\hline 2 & 1 & 268 & $1,609.9$ & $1,306.4$ & $<$ & $1,609.9$ & $1,609.9$ \\
\hline 2 & 2 & 231 & $1,339.9$ & $1,609.9$ & $>$ & $1,339.9$ & $1,609.9$ \\
\hline 3 & 1 & 200 & $1,706.7$ & $1,609.9$ & $<$ & $1,706.7$ & $1,706.7$ \\
\hline 3 & 2 & 169 & $1,408.3$ & $1,706.7$ & $>$ & $1,408.3$ & $1,706.7$ \\
\hline 4 & 1 & 162 & $1,744.0$ & $1,706.7$ & $<$ & $1,744.0$ & $1,744.0$ \\
\hline 4 & 2 & 134 & $1,425.1$ & $1,744.0$ & $>$ & $1,425.1$ & $1,744.0$ \\
\hline 5 & 1 & 137 & $1,756.2$ & $1,744.0$ & $<$ & $1,756.2$ & $1,756.2$ \\
\hline 5 & 2 & 113 & $1,420.6$ & $1,756.2$ & $>$ & $1,420.6$ & $1,756.2$ \\
\hline 6 & 1 & 120 & $1,755.3$ & $1,756.2$ & $>$ & $1,755.3$ & $1,756.2$ \\
\hline
\end{tabular}

(V1: Value 1, V2: Value 2, TP: total profit; subscript s = system, 3 = Scenario 3)

Using the approach of Grubbström and Erdem (1999), the optimum values of $n_{i}^{*}$ for Scenario $i$, where $i=1,2$, and 3, are given using Eqs. (10-10), (10-15), and (10-19), developed in Appendix 4, respectively, where Eqs. (10-12), (10-16), and (10-20) are used to find the optimum values of $m_{i}^{*}$ for the three scenarios correspondingly. Note that in the examples below, $m_{i}^{*}=1$, because $h_{v, f}=0.54<c_{c} I_{b}=1.458$. The optimal order quantities $q_{i}^{*}$ for the three scenarios are found by substituting the values of $n_{i}^{*}$ and $m_{i}^{*}$ in Eqs. (6-6), (6-11), and (6-18), respectively. The maximum total system's profit is found by substituting the values of $n_{i}^{*}, m_{i}^{*}$, and $q_{i}^{*}$ in Eqs. (6-5), (6-10), and (6-17) for Scenarios 1, 2, and 3, respectively. The optimal solutions for the three scenarios are determined using the explanation provided following Eq. (6-6) and are summarized in Table 6-6.

Table 6-6. The optimal solutions for the three scenarios using the two approaches

\begin{tabular}{ccccccc}
\hline Scenario $i$ & Method of & $n_{i}^{*}$ & $m_{i}^{*}$ & $q_{i}^{*}$ & $T P_{s, i}\left(n_{i}^{*}, m_{i}^{*}\right)$ & Comment \\
\hline 1 & $\mathrm{G} \& \mathrm{E}$ & 4.06 & 1 & 130 & $1,382.2$ & \\
& & $\mathbf{4}$ & $\mathbf{1}$ & $\mathbf{1 3 1}$ & $\mathbf{1 , 3 8 2 . 2}$ & $\left\lfloor n_{1}^{*}\right\rfloor=\lfloor 4.06\rfloor=4$ \\
& & 5 & 1 & 110 & $1,375.6$ & $\left\lceil n_{1}^{*}\right\rceil=\lceil 4.06\rceil=5$ \\
& $\mathrm{~J} \& \mathrm{G}$ & $\mathbf{4}$ & $\mathbf{1}$ & $\mathbf{1 3 1}$ & $\mathbf{1 , 3 8 2 . 2}$ & \\
\cline { 2 - 7 } 2 & $\mathrm{G} \& \mathrm{E}$ & 4.35 & 1 & 130 & $1,482.2$ & \\
& & $\mathbf{4}$ & $\mathbf{1}$ & $\mathbf{1 3 8}$ & $\mathbf{1 , 4 8 1 . 1}$ & $\left\lfloor n_{2}^{*}\right\rfloor=\lfloor 4.35\rfloor=4$ \\
& & 5 & 1 & 116 & $1,479.2$ & $\left\lceil\mathrm{n}_{2}^{*}\right\rceil=\lceil 4.35\rceil=5$ \\
& $\mathrm{~J} \& \mathrm{G}$ & $\mathbf{4}$ & $\mathbf{1}$ & $\mathbf{1 3 8}$ & $\mathbf{1 , 4 8 1 . 1}$ & \\
& $\mathrm{G} \& \mathrm{E}$ & 5.39 & 1 & 130 & $1,757.0$ & \\
& & $\mathbf{5}$ & $\mathbf{1}$ & $\mathbf{1 3 7}$ & $\mathbf{1 , 7 5 6 . 2}$ & $\left\lfloor n_{3}^{*}\right\rfloor=\lfloor 5.39\rfloor=5$ \\
& & 6 & 1 & 120 & $1,755.3$ & $\left\lceil n_{3}^{*}\right\rceil=\lceil 5.39\rceil=6$ \\
& $\mathrm{~J} \& \mathrm{G}$ & $\mathbf{5}$ & $\mathbf{1}$ & $\mathbf{1 3 7}$ & $\mathbf{1 , 7 5 6 . 2}$ & \\
\hline
\end{tabular}

G\&E: Grubbström and Erdem (1999) and J\&G: Jaber and Goyal (2008) 
The optimal solutions for the three scenarios using the approaches provided in Jaber and Goyal (2008; pp. 98-100) and summarized in Section 6.4 produced the same results as the approach of Grubbström and Erdem (1999). The optimal results for the two approaches, Grubbström and Erdem (1999) and Jaber and Goyal (2008), in Table 6-6, are in bold. The results show that offering a delay-in-payment to the buyer by the vendor increases the supply chain profits. Scenario 3 produced the most profit. It also shows that the optimal number of payments is $m_{i}^{*}=1$ for the three scenarios, $i=1,2$, and 3, and the lot size $\left(n_{i}^{*} q_{i}^{*}\right)$ increases when a payment is delayed; i.e., $n_{1}^{*} q_{1}^{*}=524, n_{2}^{*} q_{2}^{*}=552$, and $n_{3}^{*} q_{3}^{*}=685$.

Table 6-7 shows that the total supply chain profit increases by $7.2 \%$ when the vendor offers the buyer an interest-free delay-in-payment (comparing Scenarios 1 and 2), and by 27.1\% when the delay-in-payment period is extended (comparing Scenarios 1 and 3). It also shows the percentage differences (+/-) in the total profits and costs of the vendor and the buyer as the supply chain moves from Scenario 1 to $2(2 \rightarrow 1)$ and from 1 to $3(3 \rightarrow 1)$. For $2 \rightarrow 1$, the vendor's profit decreases by $12.6 \%$ as its cost increases by $0.8 \%$ as more money becomes tied in inventory and thus losing the opportunity to invest its revenue. The buyer benefits more than the vendor as its profit increases by $12.8 \%$. It may be possible for the buyer to compensate the vendor for its loss, which is (in dollars) $305.5-267.1=38.4$. If the buyer compensates the vendor, then its profit reduces to $\$ 1,175.6$, which is still higher than the buyer's profit for Scenario 1. When the vendor and the buyer adopt Scenario 3, $3 \rightarrow 1$, their profits increase by $8.9 \%$ and $32.2 \%$, respectively. It is not necessary for this scenario that the buyer compensates the vendor. For Scenarios 2 and 3, the vendor and the buyer may negotiate a profit sharing mechanism, which can take the form of the buyer accepting a higher purchase price. These results suggest that a longer period of a delay-inpayment is beneficial for the supply chain.

Table 6-7. Detailed comparison between the results of the three scenarios

\begin{tabular}{lccccccc} 
& $T C_{v, i}(\$)$ & $T R_{v, i}(\$)$ & $T P_{v, i}(\$)$ & $T C_{b, i}(\$)$ & $T R_{b, i}(\$)$ & $T P_{b, i}(\$)$ & $T P_{s, i}(\$)$ \\
\hline Scenario 1 & $5,094.5$ & $5,400.0$ & 305.5 & $6,595.8$ & $7,672.5$ & $1,076.7$ & $1,382.2$ \\
Scenario 2 & $5,132.9$ & $5,400.0$ & 267.1 & $6,640.8$ & $7,854.8$ & $1,214.0$ & $1,481.1$ \\
Scenario 3 & $5,244.8$ & $5,577.6$ & 332.8 & $7,045.5$ & $8,468.9$ & $1,423.3$ & $1,756.2$ \\
\hline $2 \rightarrow 1$ & $0.8 \%$ & $0.0 \%$ & $-12.6 \%$ & $0.7 \%$ & $2.4 \%$ & $12.8 \%$ & $7.2 \%$ \\
\hline $3 \rightarrow 1$ & $3.0 \%$ & $3.3 \%$ & $8.9 \%$ & $6.8 \%$ & $10.4 \%$ & $32.2 \%$ & $27.1 \%$ \\
\hline (TC: total cost, TR: total revenue, TP: total profit; subscript $\mathrm{v}=$ vendor, $\mathrm{b}=$ buyer, $\mathrm{s}=$ system, $\mathrm{i}=1,2,3$ and represents the \\
scenarios)
\end{tabular}




\subsubsection{Comparison between the CS and the traditional policy}

This section presents a comparison between the CS and the traditional policy considering the three scenarios developed in Sections 6.1, 6.2 and 6.3. Aljazzar et al. (2016a) investigated the effect of the delay-in-payment (a decision variable) on three different traditional policies for a two-level supply chain, which are the models of Goyal (1988), Hill (1997), and Jaber et al. (2010). They concluded that the model of Hill (1997) performed better than the other two. In this regard, this chapter adopts the model of Hill (1997), and modifies its total cost function to consider the three scenarios of Sections 6.1, 6.2 and 6.3.

Accordingly, the first scenario of Hill (1997) assumes that a delay-in-payment is not offered by the vendor and the buyer pays for the shipment whenever it is received. After modifying Eq. (9) of Hill (1997; p. 496) the total supply chain profit is written as

$$
\begin{aligned}
& T P_{s, 1}^{H i l l}\left(q_{1}, n_{1}\right)=\left(c_{c}-\gamma c_{p}-c_{p r}\right) D-\left(S_{v}+n_{1} O_{b}+n_{1} c_{t}\right)\left(\frac{D}{n_{1} q_{1}}\right)-h_{v}\left(\frac{q_{1} D}{P}+\right. \\
& \left.\frac{(P-D) n_{1} q_{1}}{2 P}\right)-\left(h_{b}-h_{v}\right) \frac{q_{1}}{2}
\end{aligned}
$$

The optimal quantity that maximizes Eq. (6-19) is

$$
q_{H i l l, 1}\left(n_{1}\right)=\sqrt{\frac{\left(S_{v}+n_{1} O_{b}+n_{1} c_{t}\right)\left(\frac{D}{n_{1}}\right)}{h_{v}\left(\frac{D}{P}+\frac{(P-D) n_{1}}{2 P}\right)+\frac{\left(h_{b}-h_{v}\right)}{2}}}
$$

The second scenario of Hill (1997) considers that the vendor offers the buyer a permissible period to settle its balance at no additional cost. The buyer pays the vendor by the end of the permissible period. The total supply chain profit for the model of Hill (1997) is written as

$$
\begin{aligned}
& T P_{s, 2}^{H i l l}\left(q_{2}, n_{2}\right)=\left(c_{c}-\gamma c_{p}-c_{p r}\right) D-\left(S_{v}+n_{2} O_{b}+n_{2} c_{t}\right)\left(\frac{D}{n_{2} q_{2}}\right)-h_{v}\left(\frac{q_{2} D}{P}+\right. \\
& \left.\frac{(P-D) n_{2} q_{2}}{2 P}\right)-\left(h_{b}-h_{v}\right) \frac{q_{2}}{2}+\alpha\left(c_{c} I_{b}-c_{b} I_{v}\right) q_{2}
\end{aligned}
$$

The optimal quantity that maximizes Eq. (6-21) is 


$$
q_{H i l l, 2}\left(n_{2}\right)=\sqrt{\frac{\left(S_{v}+n_{2} O_{b}+n_{2} c_{t}\right)\left(\frac{D}{n_{2}}\right)}{h_{v}\left(\frac{D}{P}+\frac{(P-D) n_{2}}{2 P}\right)+\frac{\left(h_{b}-h_{v}\right)}{2}-\alpha\left(c_{c} I_{b}-c_{b} I_{v}\right)}}
$$

Note that, when $\alpha=0$, Eqs. (6-21) and (6-22) reduce to Eqs. (6-19) and (6-20), respectively.

Scenario 3 for Hill (1997) considers a delay-in-payment where the buyer pays the invoice post the interest-free delay period and incurs cost from interest charged by the vendor. The supply chain system's total profit is written as

$$
\begin{aligned}
& T P_{s, 3}^{\text {Hill }}\left(q_{3}, n_{3}\right)=\left(c_{c}-\gamma c_{p}-c_{p r}\right) D-\left(S_{v}+n_{3} O_{b}+n_{3} c_{t}\right)\left(\frac{D}{n_{3} q_{3}}\right)-h_{v}\left(\frac{q_{3} D}{P}+\right. \\
& \left.\frac{(P-D) n_{3} q_{3}}{2 P}\right)-\left(h_{b}-h_{v}\right) \frac{q_{3}}{2}+\left(c_{c} I_{b}-c_{b} I_{v}\right)(\alpha+\beta(1+\alpha)) q_{3}
\end{aligned}
$$

The optimum quantity that maximizes Eq. (6-23) is

$$
q_{H i l l, 3}\left(n_{3}\right)=\sqrt{\frac{\left(S_{v}+n_{3} O_{b}+n_{3} c_{t}\right)\left(\frac{D}{n_{3}}\right)}{h_{v}\left(\frac{D}{P}+\frac{(P-D) n_{3}}{2 P}\right)+\frac{\left(h_{b}-h_{v}\right)}{2}-\left(c_{c} I_{b}-c_{b} I_{v}\right)(\alpha+\beta(1+\alpha))}}
$$

Note that, when $\alpha=\beta=0$, Eqs. (6-23) and (6-24) reduce to Eqs. (6-19) and (6-20), respectively.

The three versions of Hill's model presented above are solved (using the approach of Jaber and Goyal, 2008) for the input parameters provided in Section 6.5.1. The results are summarized in Table 6-8.

Table 6-8. Comparison between the CS and Hill (1997) models with/without the delay-in-payment

\begin{tabular}{cccccc}
\hline Scenario $i$ & Policy & $n_{i}^{*}$ & $m_{i}^{*}$ & $q_{i}^{*}\left(n_{i}, m_{i}\right)$ & $T P_{s, i}\left(n_{i}, m_{i}, q\left(n_{i}, m_{i}\right)\right)(\$)$ \\
\hline 1 & CS & 4 & 1 & 131 & $1,382.2$ \\
& Hill & 4 & 4 & 132 & $1,382.3$ \\
2 & CS & 4 & 1 & 138 & $1,481.1$ \\
& Hill & 4 & 4 & 133 & $1,406.6$ \\
3 & CS & 5 & 1 & 137 & $1,756.2$ \\
& Hill & 4 & 4 & 138 & $1,466.2$ \\
\hline
\end{tabular}

(CS: consignment stock, Hill: modified model of Hill (1997), TP: total profit; subscript $s=$ system, $i=1,2,3$ and represents the scenarios) 
The results in Table 6-8 show that both the CS and Hill models returned the same supply chain system's total profit for Scenario 1. The CS policy produced higher profits for Scenarios 2 and 3, suggesting that CS (for these values of parameters) is more profitable than a traditional policy. These results confirm several instances where CS was reported to perform better than the traditional coordination policy.

To have a broader view of how the supply chain system behaves for the three scenarios of the CS and Hill models, 5,000 set of the input parameters were generated randomly, and the optimal results were found for the three scenarios using a nested loop search (See Jaber and Goyal, 2008; pp. 98-100) written in Visual Basic for Applications (VBA). Table 6-9 shows the values of the minimum and the maximum ranges, and the average and the standard deviation of the generated examples for each input parameter.

Table 6-9. Minimum and maximum ranges, and average and standard deviation values for each parameter for 5,000 numerical examples

\begin{tabular}{|c|c|c|c|c|}
\hline \multirow{2}{*}{ Parameter } & \multicolumn{2}{|c|}{ Range } & \multirow{2}{*}{$\begin{array}{c}\text { Average of } \\
\text { generated examples }\end{array}$} & \multirow{2}{*}{$\begin{array}{l}\text { Standard deviation of } \\
\text { generated examples }\end{array}$} \\
\hline & Minimum & Maximum & & \\
\hline$P$ & $1,000.00$ & $11,000.00$ & $7,351.40$ & $2,368.44$ \\
\hline$D$ & $1,000.00$ & 6000.00 & $3,099.36$ & $1,395.56$ \\
\hline$S_{v}$ & 100.00 & 800.00 & 452.89 & 201.14 \\
\hline$O_{b}$ & 10.00 & 200.00 & 108.91 & 57.27 \\
\hline$h_{v, f}$ & 0.00 & 3.76 & 1.38 & 0.82 \\
\hline$h_{v, S}$ & 1.00 & 4.00 & 2.51 & 1.11 \\
\hline$h_{b, s}$ & 3.00 & 8.00 & 5.62 & 1.70 \\
\hline$\gamma$ & 1.00 & 1.00 & 1.00 & 0.00 \\
\hline$\alpha$ & 0.00 & 1.00 & 0.47 & 0.28 \\
\hline$\beta$ & 0.00 & 1.00 & 0.46 & 0.28 \\
\hline$I_{v}$ & 0.00 & 0.40 & 0.23 & 0.11 \\
\hline$I_{b}$ & 0.00 & 0.40 & 0.16 & 0.10 \\
\hline$c_{p}$ & 1.00 & 4.00 & 2.43 & 1.12 \\
\hline$c_{p r}$ & 1.00 & 3.00 & 1.93 & 0.81 \\
\hline$c_{b}$ & 2.70 & 9.45 & 5.88 & 1.88 \\
\hline$c_{c}$ & 3.65 & 12.76 & 7.94 & 2.54 \\
\hline$c_{t}$ & 0.50 & 1.00 & 0.90 & 0.06 \\
\hline
\end{tabular}

As it is difficult to observe the effects of parameters from the closed-form expressions that were developed and a closed-form expression for the total profits were not found, a regression analysis was performed on the results to indicate how the total profit is affected by changing the input 
parameters. The results showed that for CS Scenarios 1 and 3, the significant parameters are $P, D, S_{v}, O_{b}, h_{v, f}, h_{v, s}, h_{b, s}, \alpha, \beta, I_{v}, I_{b}, c_{p}$ and $c_{c}$ for $p$-values $<5 \%$. For CS Scenario 2, the significant parameters are $P, D, S_{v}, O_{b}, h_{v, f}, h_{v, s}, h_{b, s}, \alpha, I_{v}, I_{b}, c_{p}$ and $c_{c}$ for $p$-values $<5 \%$. For Hill Scenarios 1, 2 and 3, the significant parameters are $P, D, S_{v}, O_{b}, h_{v, f}, h_{b, s}, \alpha, \beta, I_{v}, I_{b}, c_{p}$ and $c_{c}$ for $p$-values $<5 \%$. Table 6-10 represents the coefficients' values of the significant parameters ( $p$ values $<5 \%$ ) for the three scenarios for the CS and Hill models. A positive coefficient value shows how much the total profit increases when a parameter increases by one unit where a negative coefficient shows the reduction in the total profit when the related parameter increases by one unit. The coefficients' values of the insignificant parameters ( $p$-values $>5 \%$ ) were excluded from the table or marked by (-) mark as their effects are minimal.

Table 6-10. Coefficients' values of the significant parameters for CS and Hill models

\begin{tabular}{lllllll}
\cline { 2 - 7 } Parameter & \multicolumn{6}{c}{ Coefficients } \\
\cline { 2 - 7 } & CS 1 & CS 2 & CS 3 & Hill 1 & Hill 2 & Hill 3 \\
\hline$P$ & -0.1 & -0.1 & -0.1 & -0.1 & -0.1 & -0.1 \\
$D$ & 3.7 & 3.7 & 3.8 & 3.7 & 3.7 & 3.7 \\
$S_{v}$ & -2.8 & -2.7 & -2.3 & -1.7 & -1.7 & -1.7 \\
$O_{b}$ & -9.0 & -9.2 & -9.4 & -9.9 & -9.8 & -9.5 \\
$h_{v, f}$ & $-1,513.4$ & $-1,581.4$ & $-1,628.5$ & $-1,177.7$ & $-1,275.1$ & $-1,371.1$ \\
$h_{v, s}$ & 282.3 & 284.4 & 279.0 & - & - & - \\
$h_{b, s}$ & -559.5 & -565.7 & -564.0 & -409.6 & -411.2 & -414.1 \\
$\alpha$ & - & 935.2 & 1059.3 & - & 728.5 & 783.1 \\
$\beta$ & - & - & 986.1 & - & - & 726.5 \\
$I_{v}$ & $-6,463.1$ & $-7,245.5$ & $-9,247.4$ & $-5,171.2$ & $-5,335.8$ & $-5,799.5$ \\
$I_{b}$ & $13,116.7$ & $14,771.7$ & $18,064.8$ & $11,178.0$ & $12,224.7$ & $13,755.5$ \\
$c_{p}$ & 755.5 & 759.7 & 749.8 & 757.5 & 759.3 & 751.0 \\
$c_{c}$ & $1,629.8$ & $1,657.9$ & $1,701.3$ & $1,599.5$ & $1,616.9$ & $1,639.6$ \\
\hline
\end{tabular}

Table 6-11 (next page) shows the number and the percentage of examples (from 5,000 examples) that achieved the highest system's profit for each scenario under both policies. The results show that Scenario 3 for the CS policy returned the highest system's profit in 1,975 examples where Hill policy returned the highest in 1,520. For the rest of the results, see Table 6-11. This confirms that choosing the best policy depends on the values of the parameters. 
Table 6-11. Number and percentages of examples that returned the highest system's profit for 5,000 examples

\begin{tabular}{lccc|ccc} 
& \multicolumn{3}{c|}{ Consignment Stock } & \multicolumn{3}{c}{ Hill model } \\
\cline { 2 - 7 } & Scenario 1 & Scenario 2 & Scenario 3 & Scenario 1 & Scenario 2 & Scenario 3 \\
\hline $\begin{array}{l}\text { Total no. of examples } \\
\text { achieved the highest profit }\end{array}$ & 10 & 3 & 1,975 & 1,481 & 11 & 1,520 \\
$\begin{array}{l}\text { Percentage of examples } \\
\text { achieved the highest profit }\end{array}$ & $0.2 \%$ & $0.1 \%$ & $39.5 \%$ & $29.6 \%$ & $0.2 \%$ & $30.4 \%$ \\
\hline
\end{tabular}

To understand the effects of some of the input parameters on choosing the best policy that serves the system, the sensitivity study is provided in the next section.

\subsection{Sensitivity Analysis}

This section provides a sensitivity analysis performed on the CS and the modified model of Hill (1997) considered above. When investigating the sensitivity of one input parameter, the values of the other parameters in Section 6.5.1 were kept unchanged.

Figure 6-4 shows that as the ratio of the demand rate to the production rate, $D / P$, increases from 0.1 to 0.6 , the profit of the supply chain increases for the CS and Hill models for the three scenarios. For Scenario 1, Hill's model returns higher total profit when $0.1 \leq D / P<0.4$ and the CS when $D / P \geq 0.4$. The results show that the CS is more profitable than Hill's model for Scenarios 2 and

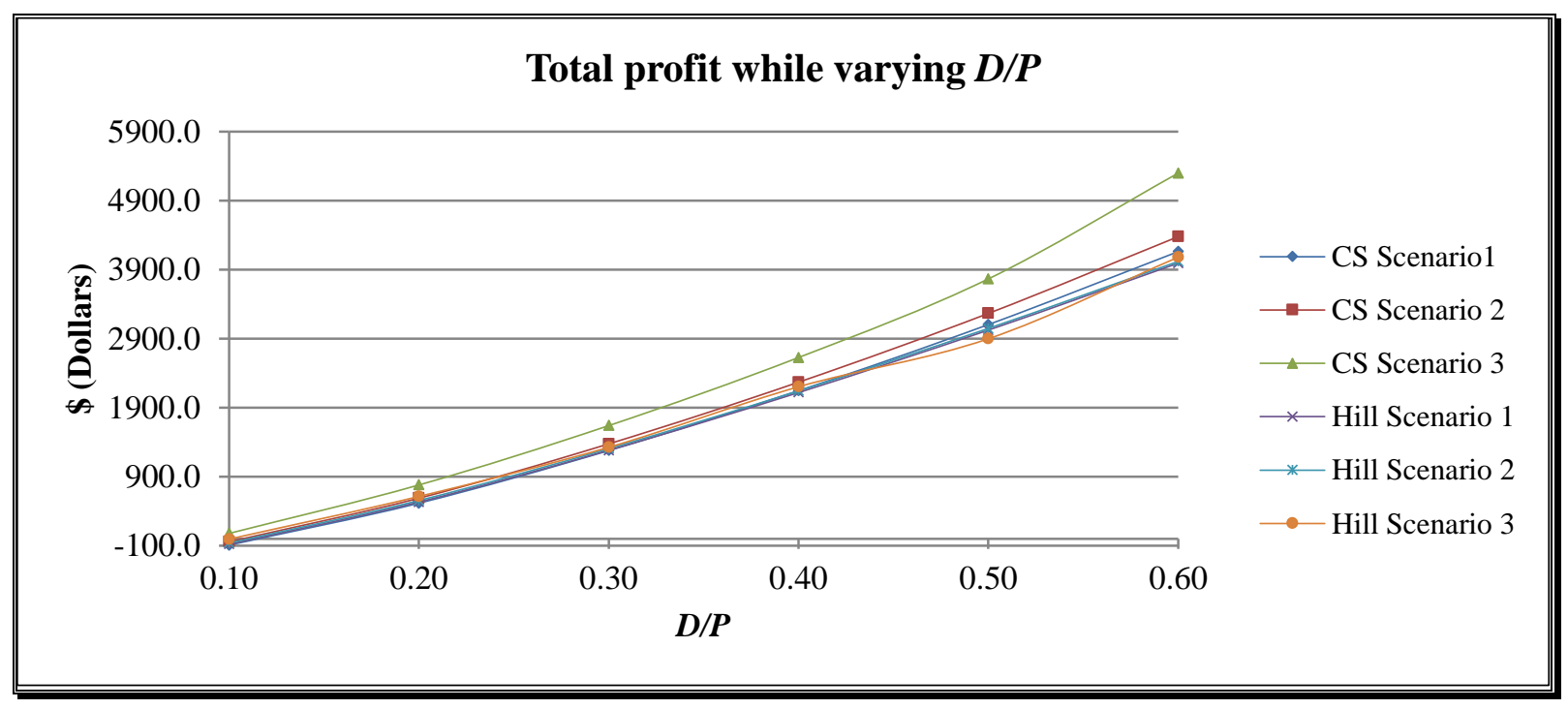

Figure 6-4. Total profit while varying $D / P$ 
3. This shows that the CS policy is more flexible than Hill models as it returns higher profit for a wide range of $D / P$.

Figure 6-5 shows that as the ratio of the ordering cost of the buyer to the setup cost of the vendor, $O_{b} / S_{v}$, increases, Scenario 3 is the most profitable followed by Scenario 2 for both the CS and Hill. It also shows that the CS is more profitable than the model of Hill for the three scenarios. The results show that the total supply chain profits for the CS and Hill models decrease as $O_{b} / S_{v}$ increases from 0.2 to 1.2. This suggests that the CS policy is a more favored policy when orders are made by the buyer electronically at a very low cost.

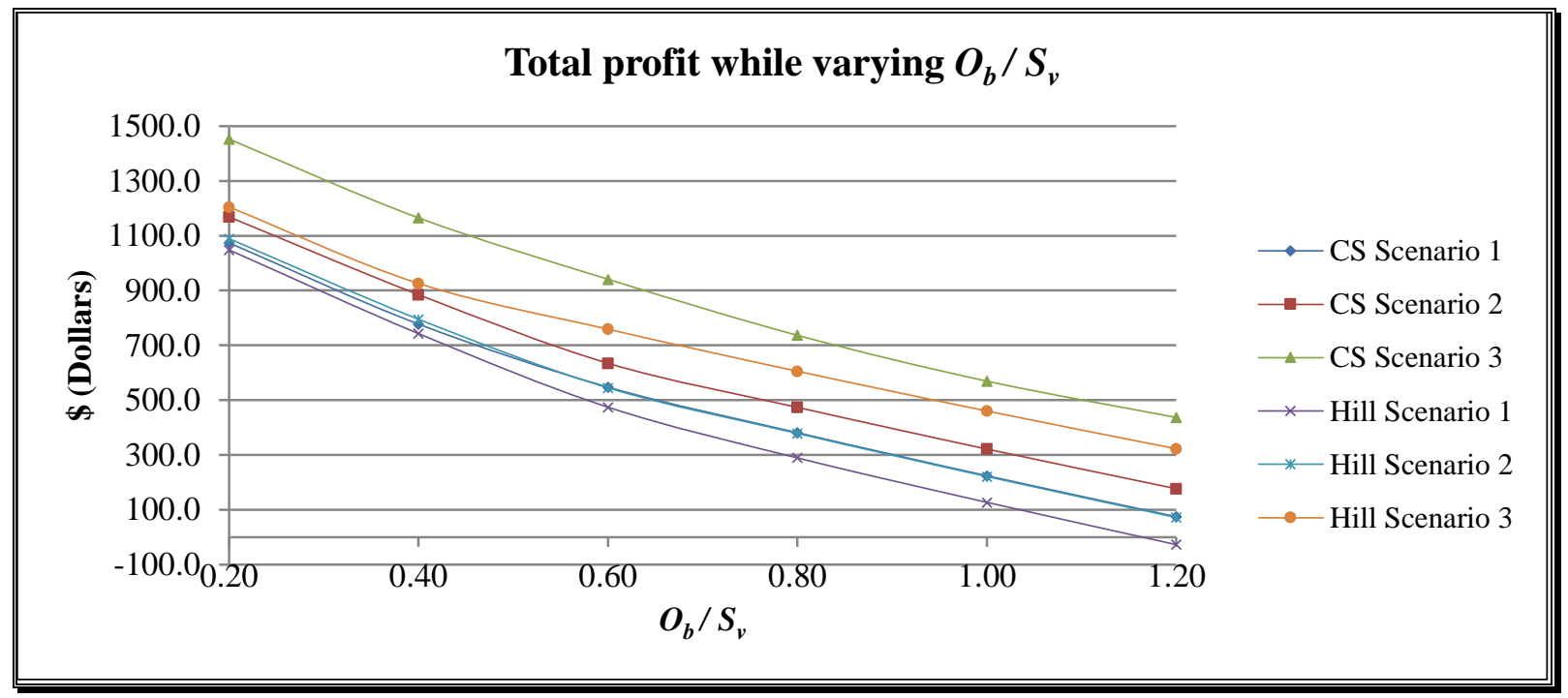

Figure 6-5. Total profit while varying $O_{b} / S_{v}$

Varying the ratio of the financial-holding cost of the vendor to its storage-holding cost, $h_{v, f} / h_{v, s}$, between 0.2 and 1.2 for the CS and Hill policies show (in Figure 6-6 next page) that the supply chain total profit for Hill is higher than the CS model for Scenarios 1 and 2 for all tested points. For Scenario 3, the CS returns higher profit than Hill when $h_{v, f} / h_{v, s} \leq 0.24$ where Hill returns higher profit for the remaining values of $h_{v, f} / h_{v, s}$. These and the other results in Figure 6-6 suggest that CS performs better than Hill when it is expensive to store items at the vendor's side, $h_{v, s}$, and significantly higher than the cost of capital tied up in inventory, $h_{v, f}$. 


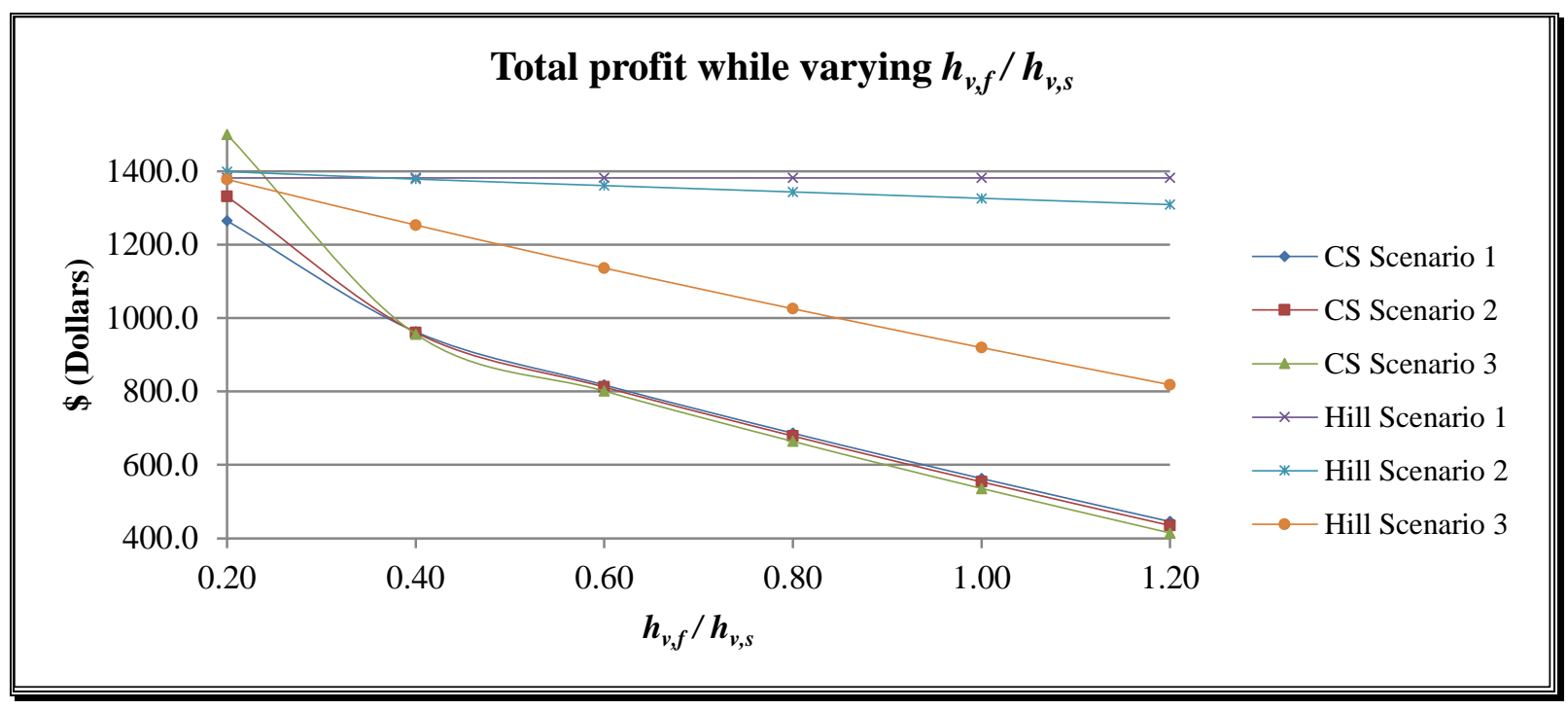

Figure 6-6. Total profit while varying $h_{v, f} / h_{v, s}$

Figure 6-7 shows that Scenario 3 is the most profitable whereas Scenario 1 has the worst performance for both the CS and Hill policies, when the ratio of the storage-holding cost of the buyer to the storage-holding cost of the vendor, $h_{b, s} / h_{v, s}$, is varied from 0.75 to 1.5 . The results show that the total profits for both models and all scenarios decrease as $h_{b, s} / h_{v, s}$ increases with the differences between the total profits for the three scenarios becoming very small. This suggests that a CS is more profitable and recommended when the storage-holding cost for the buyer is less than the storage-holding cost for the vendor.

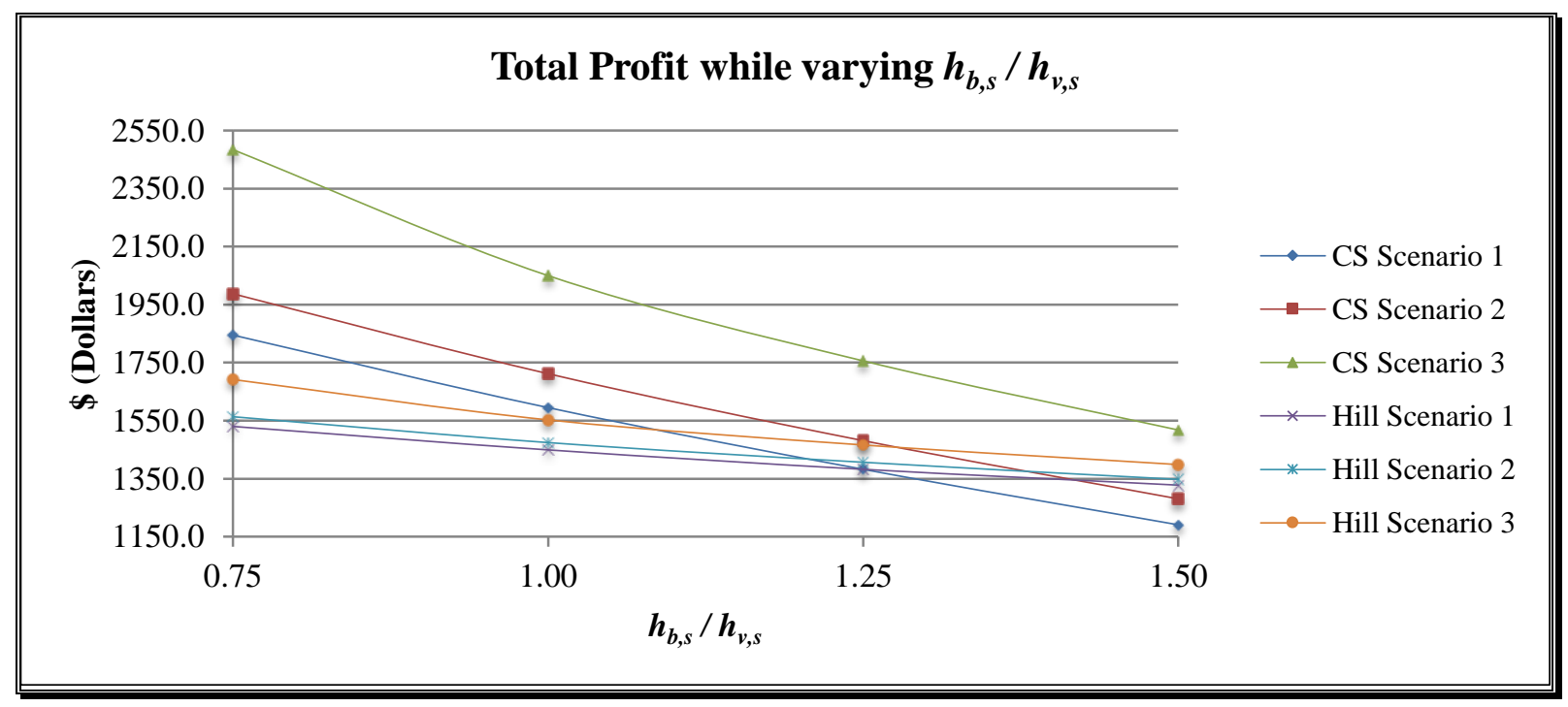

Figure 6-7. Total profit while varying $h_{b, s} / h_{v, s}$ 
In this part of the analysis, the total profits for the developed scenarios are calculated for increasing the length of the interest-free and the interest-charge periods (i.e., by increasing $\alpha$ from 0 to 0.6 and $\beta / \alpha$ from 0 to 1.6). The results in Figure 6-8 show that as the length of the interest-free delay period increases by $20 \%(\alpha=0.2)$, the total supply chain profits for the CS policy (Hill) for Scenarios 2 and 3 increase by about 7.2\% (1.8\%) and 10.5\% (2.4\%), respectively. Figure 6-9, which applies for Scenario 3, shows that as the length of the non-interest free period increases relative to the interest-free period, i.e. $\beta / \alpha$, the supply chain profits for the CS and Hill's model increase with CS performing significantly better. This suggests that when a delay-in-payment is applied, it is better that the supply chain moves to CS coordination.

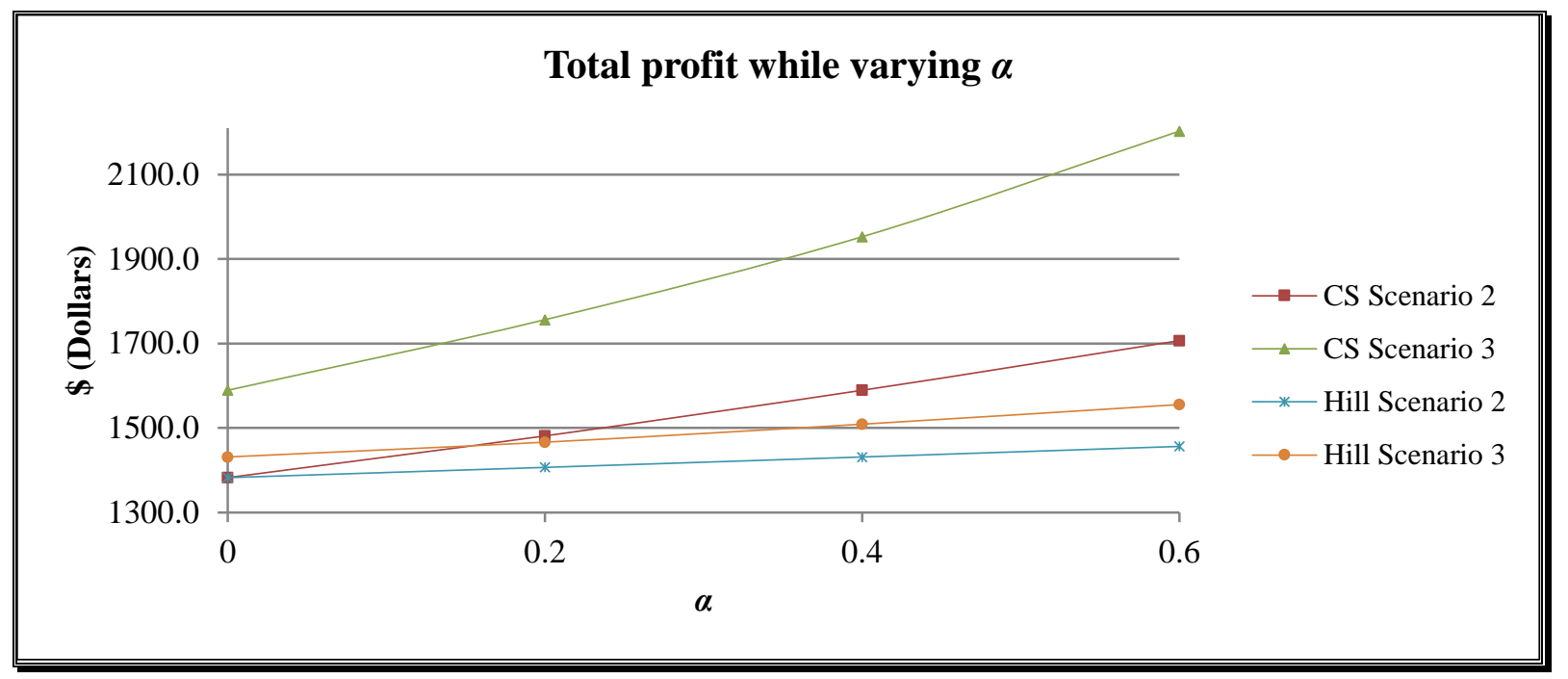

Figure 6-8. Total profit while varying $\alpha$

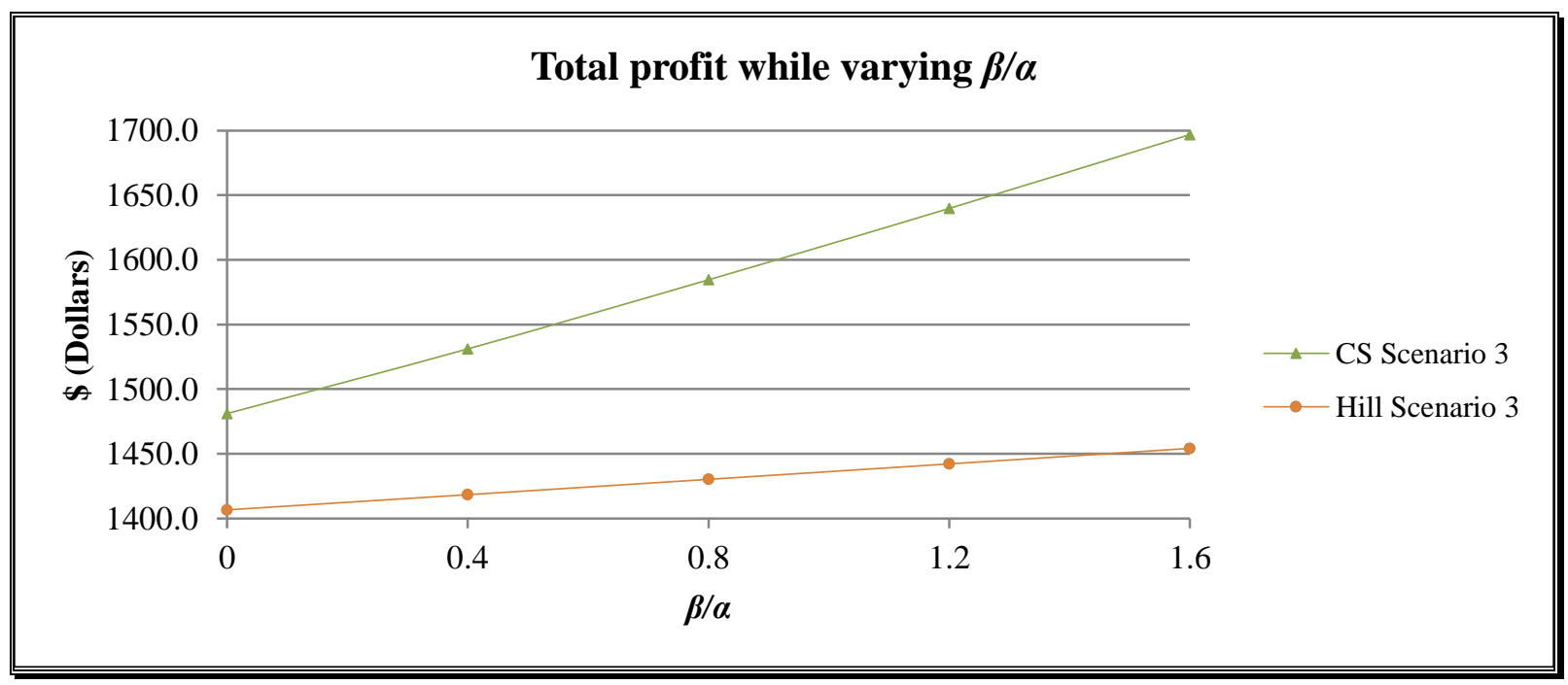

Figure 6-9. Total profit while varying $\beta / \alpha$ 
Figure 6-10 shows the behavior of the supply chain profits for the ratio of the return on investment for the buyer to that of the vendor, $I_{b} / I_{v}$. The results showed that the CS policy performed better than that of Hill when $I_{b} / I_{v}>2, I_{b} / I_{v} \geq 1.709$ and $I_{b} / I_{v} \geq 1.365$ for Scenarios 1,2 and 3 , respectively. This is because when CS is adopted, the buyer loses some of its profit due to an increase in its costs, especially, the storage-holding. If the delay-in-payments is offered to the buyer and the buyer pays after not by the date a payment is due, then, it has a longer time to invest its sales revenue and earn more money.

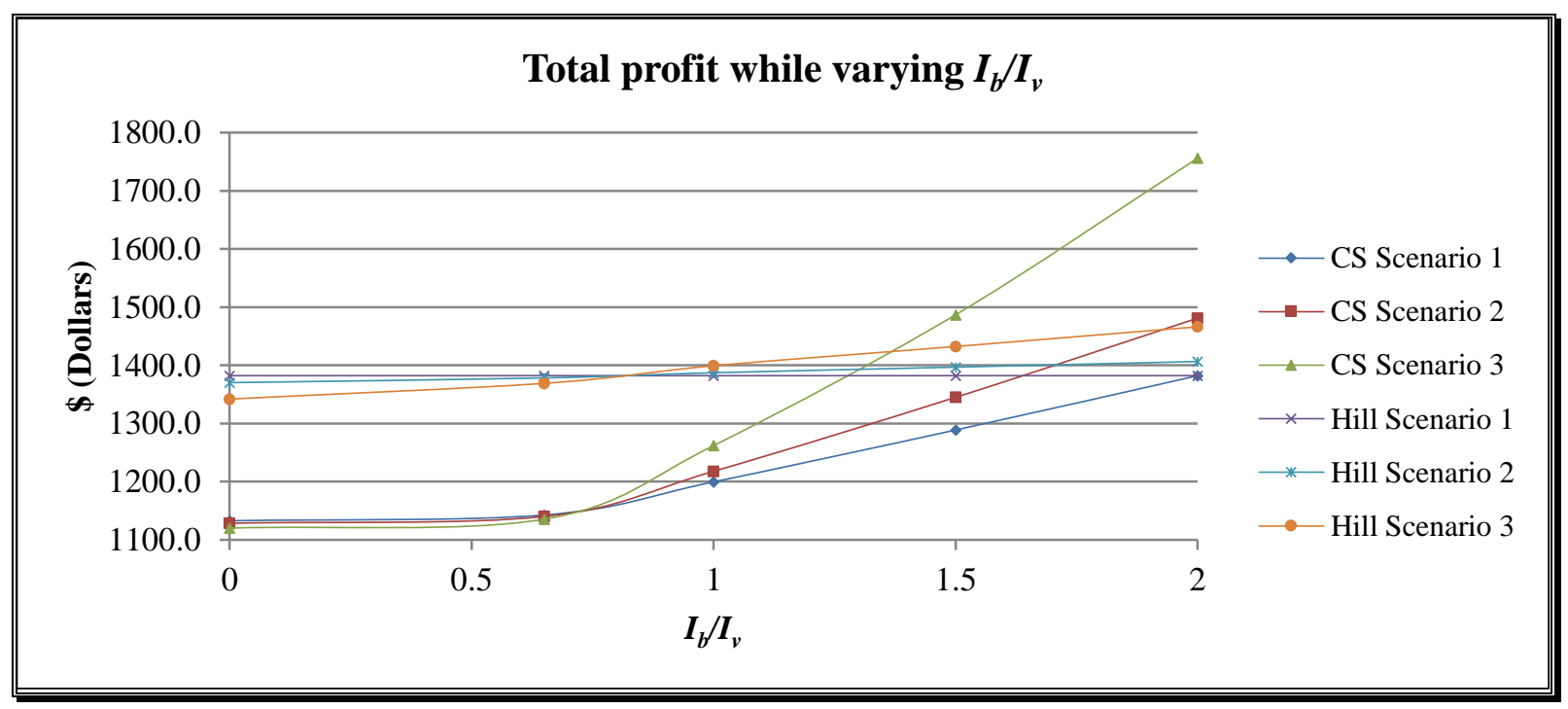

Figure 6-10. Total profit while varying $I_{b} / I_{v}$

In summary, a vendor and a buyer coordinating with the CS policy or Hill's policy reap the most benefits from their co-operation if they adopt Scenario 3. However, for Scenario 3 to record the most profit when the CS is adopted, it is recommended that the supply chain have the following: $D / P, \alpha, \beta / \alpha$ and $I_{b} / I_{v}$ are high, and $O_{b} / S_{v}, h_{v, f} / h_{v, s}, h_{b, s} / h_{v, s}$ are low. For Hill policy, Scenario 3 records the highest profit when $D / P, \alpha, \beta / \alpha$ and $I_{b} / I_{v}$ are high, and the ratio of $O_{b} / S_{v}$ and $h_{b, s} / h_{v, s}$ are low.

\subsection{Managerial Insights}

This section provides some general insights to help managers and/or decision makers when making a decision.

1. The delay-in-payment has a positive impact on the system when it is incorporated into a consignment contract. 
2. The system generates the highest profit when the downstream player pays after the permissible period. As this affects the upstream player financially, the upstream player has to charge the downstream player high interest rate for the delayed payments. This forces the downstream player to settle down its account. If charging high interest rate did not help, the upstream player has to stop sending new shipments until it receives the previous payment.

3. Although a delay-in-payment helps increasing the profit of the system, the financial department for the upstream player has to set a maximum delay period that cannot be exceeded. This also can be done by maximizing the profit while setting the delay period as a decision variable and search for the optimum period. It is better to consider all factors that affect the financial situations for the upstream player.

4. As downstream player might exceed the delay period, it is better to account that when setting the maximum delay period by the upstream player.

5. Although consignment stock showed to perform better than the traditional policy, it is the responsibility of the manager to choose the best policy that serves its system by calculating the exact value of the parameters.

6. If the ordering cost of the downstream player is high and the storage-holding cost is low, it is better for the downstream player as well as the system to sign a consignment agreement as it helps in reducing the ordering cost and the loss that might occur in the profit can be compensated from the extra profit that is generated by the system.

7. As consignment stock contract affects the financial stability of the upstream player, it is always better for the upstream player to adopt the traditional policy if its financial-holding cost is high or if it is not stable financially.

8. Paying after the permissible period is not recommended if the interest rate of the upstream player is higher than the interest rate of the downstream one. This is because the downstream player ends up paying more than what it generates from investment. The downstream player might still be able to pay beyond the permissible period if and only if it can calculate the break point that it should not exceed. 


\subsection{Conclusion}

This chapter investigated a two-level supply chain system with CS policy where the buyer makes equal-sized payments to the vendor at equal intervals with the option of delay-in-payments. This is a common business practice in CS agreements. The literature showed that delay-in-payments as an incentive scheme has not been investigated in a CS context. This chapter makes a contribution in this regard. Three models were developed. The first presents an equal payment scheme with no delay-in-payments, a revised version of the one developed in Chapter 5. The second and third models build on the first model to include delay-in-payments, one when a payment is made by the permissible period (interest-free) and the second when it is made post the permissible period (interest is charged). Closed-form expressions to determine the optimum numbers of shipments and payments and the batch sizes for the three models were developed. The problems were solved using both the closed-form expressions derived in this chapter and a solution procedure from the literature (Jaber and Goyal, 2008) and presented in Section 6.4, which exactly returned the same results. Managerial insights were provided to help managers in making the right decision that maximizes their system's profit.

The numerical results showed that a CS brings additional savings to the supply chain system when a delay-in-payment is applied as an incentive. They also showed that the performance of the system is further improved when the buyer pays after the permissible period (i.e. the buyer pays interest). The best performance occurred when the buyer makes a single delayed payment to the vendor. This benefits the buyer by allowing it to invest its revenue for a longer period and the vendor by charging interest on the unpaid balance and shipping larger quantities to the buyer. However, the performance of the buyer and the vendor is affected by the interest difference between the two. So, it is always recommended that the vendor and the buyer agree to some sort of a profit sharing mechanism to compensate the losing partner in the chain.

To gain better insights into the three CS scenarios developed in this chapter, a comparison of these scenarios with a traditional policy (Hill, 1997) after modifying it and presenting three versions of it that are comparable with the three CS scenarios developed in the chapter. The results showed that the CS is more flexible and returns higher system profits under most conditions. However, choosing the best coordination policy depends on the exact values of the parameters as the traditional policy might be the best option. 
Five thousand sets of the values of the input parameters were randomly generated. Each set was used to find the optimum values of the number of shipments, number of payments and the sizes of shipments for the three developed models/scenarios for both the CS and Hill policies. A regression analysis was performed to study the effects of the parameters on the outputs of the models. The results of the analysis showed the models produce the highest profits when the values of the demand and the buyer's interest rates, the vendor's storage-holding and raw material purchasing costs, the length of a delay-in-payment, whether interest free or charged, and the buyer's selling price are maximum, and the values of the production and the vendor's interest rates, setup, buyer's ordering, vendor's financial-holding, and buyer's storage-holding costs are minimum.

This work can be extended by treating the length of a delay-in-payment as a decision variable and to consider a three-level supply chain with multiple suppliers and retailers who are not identical. It can also be extended by considering dependent demand, non-zero lead-times, and unequal-sized and intervened and payment schedule. One more extension is to account for the net present value for each delayed payment.

The next chapter compares between different coordination scenarios in a three-level supply chain system. 


\section{Chapter 7: Comparing different coordination scenarios in a three-level supply chain system}

Supply Chain Management (SCM) is a strategy that is used to improve the internal performance of one or more individual organizations as well as the collective performance of a supply chain system. It also helps in providing better services and quality products to customers.

The inventory carrying costs in a supply chain are estimated to be between $25 \%$ and $55 \%$ of its total cost (Richardson, 1995). Coordinating orders and shipments among players in a supply chain have been shown to substantially reduce supply chain costs and increase the profitability of supply chain players. For that reason, several supply chain coordination models have been presented in the literature, such as vendor-managed-inventory (VMI) systems, collaborative planning, forecasting and replenishment (CPFR), and consignment stock (CS) (Ryu, 2006).

CS is a coordination mechanism that is used in practice and shown to be effective in increasing the customer service level and supply chain performance. Many businesses, such as hospitals, clothing and furniture retailers, some gas stations, bookstores, sports equipment and musical instruments' stores, have started to adopt CS agreements (Sarker, 2014). For example, some companies such as LBMA, Trelleborg, CARFAC Saskatchewan Visual Artists, The Newcastle upon Tyne Hospitals NHS Foundation Trust, Stone Art Manufacturing, and NASCO Distributer Sales adopt a CS contract with their partners (see Chapter 6). In a CS contract, the goods are owned by the vendor and stored at the buyer's premises. The buyer pays for items upon withdrawal from its warehouse. Some studies use VMI and CS interchangeably; however, they are somewhat different. In a CS system, the buyer decides the size and the time of a shipment, whereas, in a VMI system, it is a vendor's decision (Gümüş et al., 2008). For a review of the CS policy and the work that has been done in this area, readers are referred to Sarker (2014).

Our review of the literature confirms that apart from the thesis of Surini (2011), which is a direct extension of Braglia and Zavanella (2003) and was made available to the author by Prof. Zavanella, no published study investigates a CS in a three-level supply chain. All the CS studies in the literature investigated CS policy in a two-level supply chain system with a single buyer or multibuyers. Surini (2011) considered a vendor who sells a product to a wholesaler, a cross-docking facility, who sells it to a buyer. The study compared the traditional coordination policy with the 
CS policy by considering centralized and decentralized decision-making for two scenarios: (1) the vendor pays the financial-holding cost for both the wholesaler and the buyer, and (2) the vendor pays the wholesaler financial-holding cost and the latter pays the buyer. The study of Surini focused on finding the best option of paying the financial-holding part that minimizes the total cost of the system.

To the best of the author's knowledge, no study in the literature has investigated the CS policy in a three-level supply chain system. This chapter addresses this limitation and develops a three level supply chain system consisting of a supplier (produces raw material), a vendor (stores raw material and produces a finished product) and a buyer with different coordination policies. When having more than two-levels, it may be possible that a CS policy is not employed by all players, only between adjacent ones. For that reason, this chapter studies and compares between the effect of having different agreements (a CS and no-CS) at the same time between the parties by developing four different scenarios. It also considers equal payment schemes (Chapter 5) between adjacent players in the supply chain. The four scenarios, which are developed in this chapter, are:

1. There is a consignment agreement between the supplier and the vendor, and between the vendor and the buyer; C-C Scenario.

2. There is no consignment agreement between the supplier and the vendor, but between the vendor and the buyer; NC-C Scenario.

3. There is a consignment agreement between the supplier and the vendor, but not between the vendor and the buyer; C-NC Scenario.

4. There is no consignment agreement between the supplier and the vendor, or between the vendor and the buyer; NC-NC Scenario.

The following assumptions were considered (in line with Braglia and Zavanella, 2003) when developing the models:

1. The buyer's demand is known and constant. 
2. The production rate of the supplier $>$ the vendor's raw material demand rate $\geq$ the vendor's production rate $>$ the buyer's demand rate. The rationale for this assumption is to avoid shortages at the three levels.

3. The storage-holding cost of the finished product $>$ the storage-holding cost of the raw material. This is because the finished product has a higher dollar value than the raw material used in producing it.

4. In addition to the financial-holding cost for items in inventory, an upstream player incurs an opportunity (financial-holding at a downstream player) cost when adopting a CS agreement between the players. This is because, in CS, the downstream player pays for the items after they are withdrawn from the consignment inventory; i.e. once it receives an invoice from the upstream one. On the other hand, in a traditional coordination policy, a payment is made upon receiving a shipment by a downstream player (see Table 7-1 for holding cost's distribution).

5. This chapter adopts a scheme of equal-sized payments at equal time intervals (Chapter 5).

6. When CS is employed, the buyer invests its sales revenue until the payment to the vendor is due.

7. All players have a common inventory cycle.

Table 7-1. The distribution of the storage-holding and the financial-holding costs among the players for the four scenarios

\begin{tabular}{|c|c|c|c|c|}
\hline \multirow{3}{*}{ Policy } & \multicolumn{4}{|c|}{ Position of products (raw materials or finished) } \\
\hline & \multirow{2}{*}{ Supplier } & \multicolumn{2}{|c|}{ Vendor } & \multirow{2}{*}{ Buyer } \\
\hline & & Raw material & Finished product & \\
\hline $\mathrm{C}-\mathrm{C}$ & $h_{s, s}+h_{s, f_{1}}$ & $h_{s, f_{2}}+h_{v, s}^{r}$ & $h_{v, S}^{f}+h_{v, f_{1}}^{f}$ & $h_{v, f_{2}}^{f}+h_{b, s}$ \\
\hline $\mathrm{NC}-\mathrm{C}$ & $h_{s, s}+h_{s, f_{1}}$ & $h_{v, s}^{r}+h_{v, f}^{r}$ & $h_{v, S}^{f}+h_{v, f_{1}}^{f}$ & $h_{v, f_{2}}^{f}+h_{b, s}$ \\
\hline C-NC & $h_{s, s}+h_{s, f_{1}}$ & $h_{s, f_{2}}+h_{v, s}^{r}$ & $h_{v, S}^{f}+h_{v, f_{1}}^{f}$ & $h_{b, s}+h_{b, f}$ \\
\hline $\mathrm{NC}-\mathrm{NC}$ & $h_{s, s}+h_{s, f_{1}}$ & $h_{v, s}^{r}+h_{v, f}^{r}$ & $h_{v, s}^{f}+h_{v, f_{1}}^{f}$ & $h_{b, s}+h_{b, f}$ \\
\hline
\end{tabular}


The following notations were used for the decision variables:

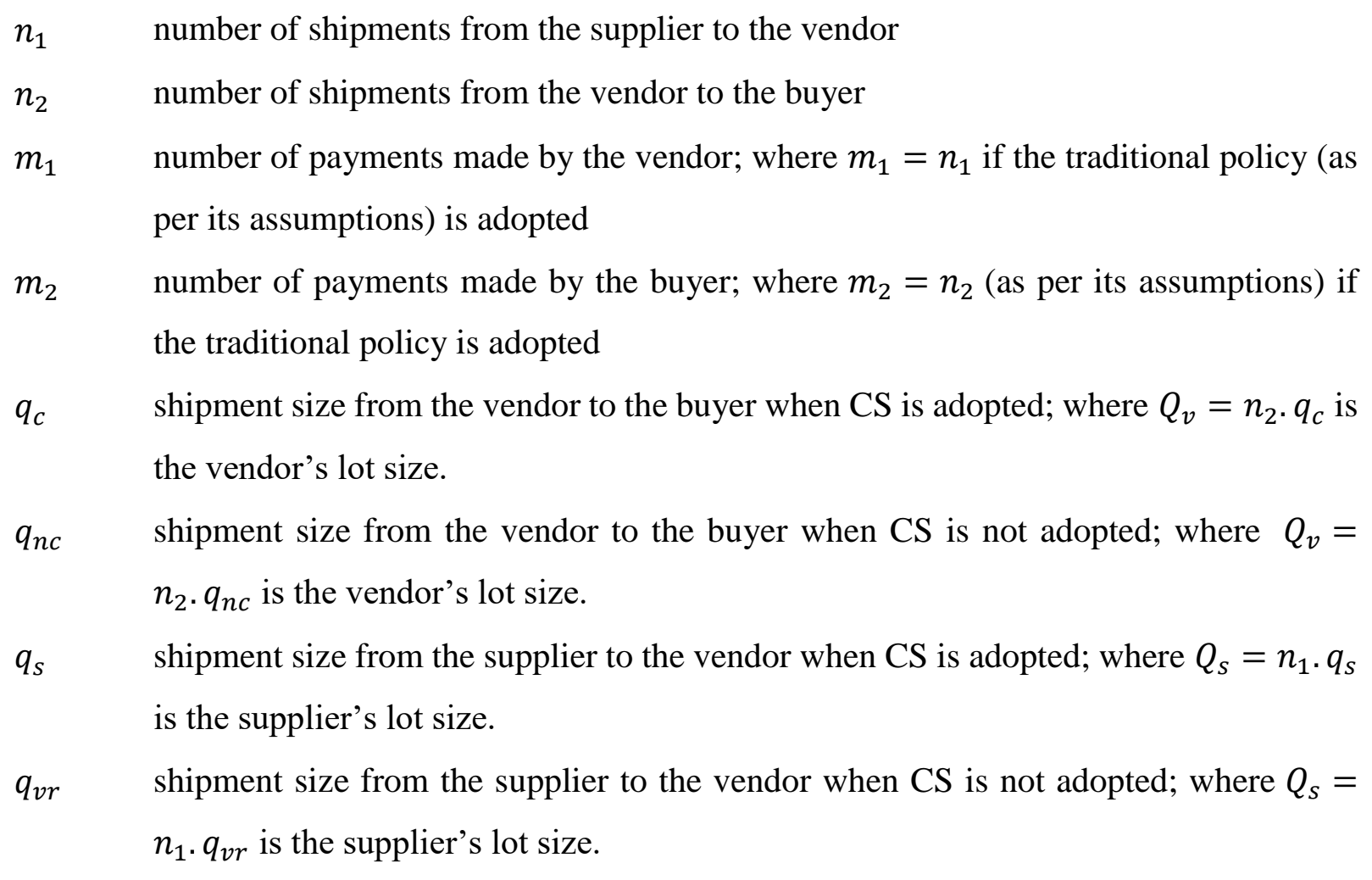

The following notations are used for the supplier:

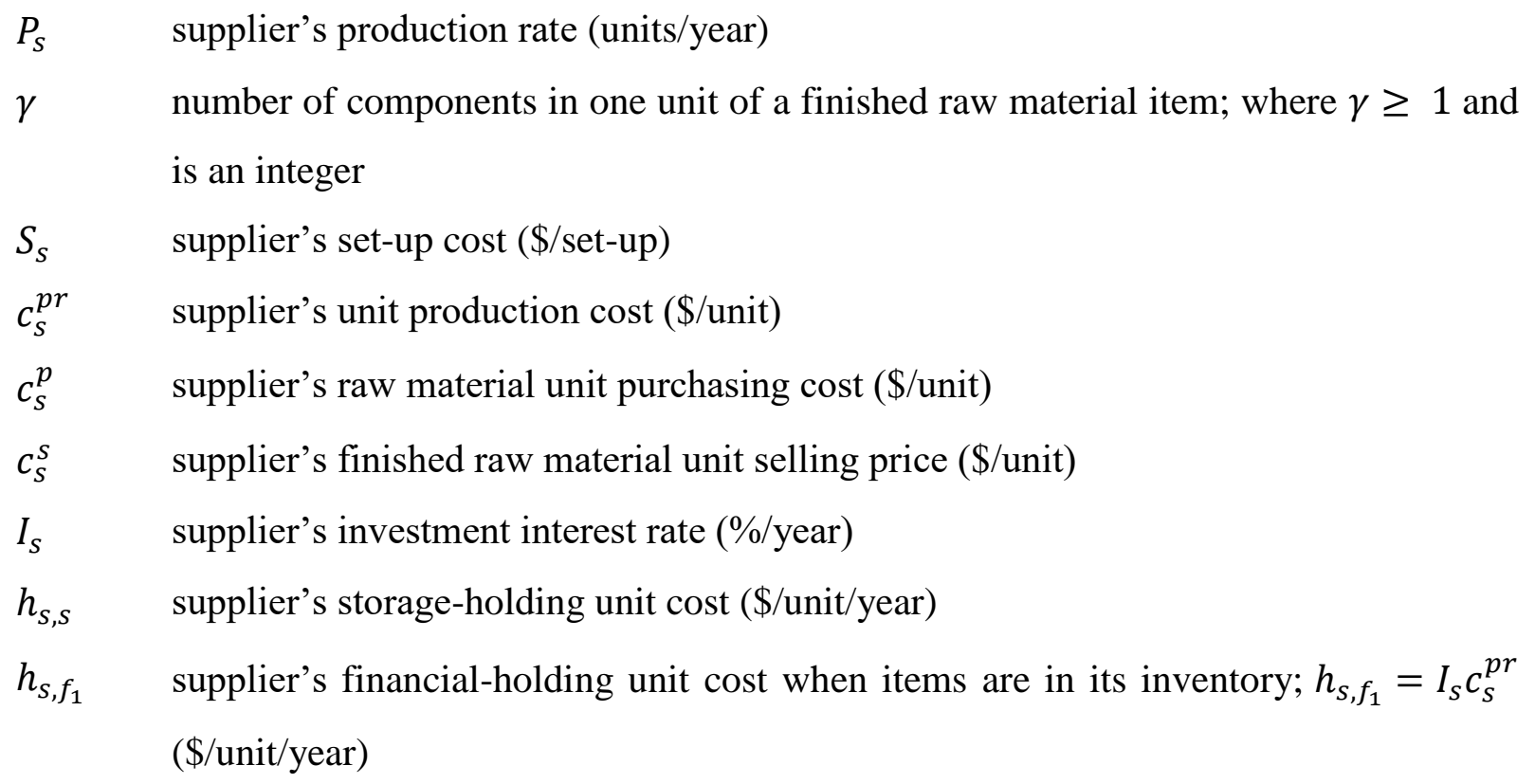


$h_{s, f_{2}} \quad$ Supplier's financial-holding unit cost when items are in the vendor's inventory; $h_{s, f_{2}}=I_{s} c_{s}^{s}(\$ /$ unit/year $)$

The following notations are used for the vendor:

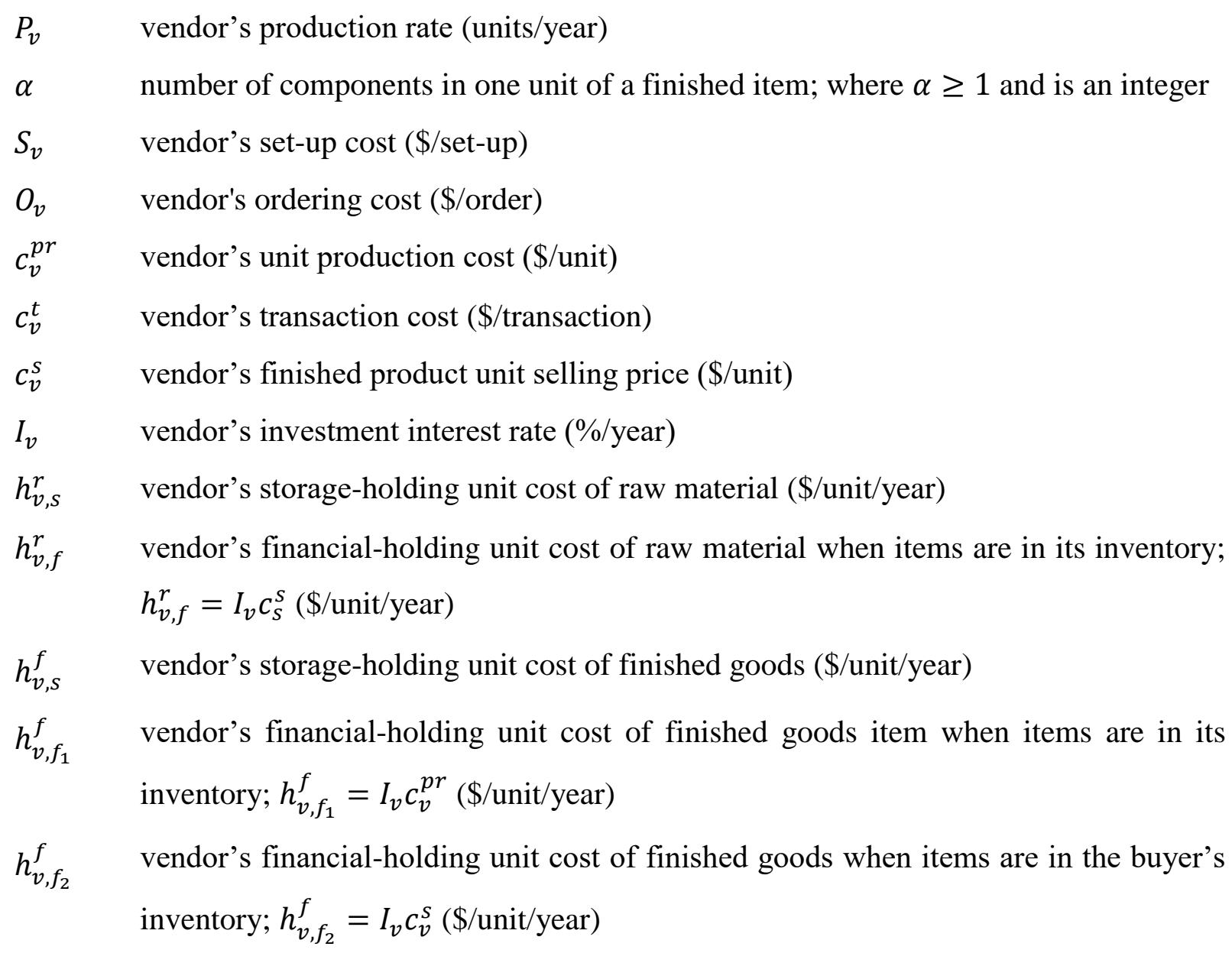

The following notations are used for the buyer:

$T \quad$ cycle time

$D \quad$ buyer's demand rate (units/year)

$O_{b} \quad$ buyer's ordering cost (\$/order)

$c_{b}^{t} \quad$ buyer's transaction cost (\$/transaction)

$c_{b}^{S} \quad$ buyer's unit selling price of a finished item (\$/unit)

$I_{b} \quad$ buyer's investment interest rate (\%/year) 
$h_{b, s} \quad$ buyer's storage-holding unit cost (\$/unit/year)

$h_{b, f} \quad$ buyer's financial-holding unit cost when items are in its inventory; $h_{b, f}=I_{b} c_{v}^{S}$ (\$/unit/year)

The rest of this chapter is organized as follows; Section 7.1 is for Scenario 1, Section 7.2 is for Scenario 2, Section 7.3 describes Scenario 3, Section 7.4 is for the fourth scenario, Section 7.5 presents a solution procedure, Sections 7.6 and 7.7 provide numerical examples and a sensitivity analysis, respectively, Section 7.8 is for managerial insights. Section 7.9 provides a summary of the chapter, presents some conclusions, and proposes some future research directions.

\subsection{Scenario 1: Consignment-Consignment (C-C) Scenario:}

This scenario assumes that all players adopt a CS agreement. The supplier produces raw material in batches and ships it to the vendor's warehouse. The vendor depletes the inventory of raw material at a constant (production) rate to produce a single product. The vendor pays for units of raw material after they are withdrawn from the consigned inventory. The vendor produces the finished product in batches and delivers it to the buyer in equal-sized shipments. The buyer pays for the withdrawn items from its inventory in equal-sized payments at equal time intervals. During this time, elapsed time between receiving a shipment and making a payment, the buyer invests its revenue from sales at some interest. The behavior of inventory for the raw material, semi-finished and finished items for the supplier, the vendor, and the buyer is shown in Figure 7-1 (next page).

The supplier generates revenue, $c_{s}^{s} n_{2} \alpha q_{c}$ per cycle, from selling the raw material to the vendor. The supplier incurs the following costs per cycle: (1) raw material purchasing, $c_{s}^{p} n_{2} \gamma \alpha q_{c}$, (2) setup, $S_{s}$, (3) production, $c_{s}^{p r} n_{2} \alpha q_{c}$, (4) the storage-holding of raw material while in inventory, $h_{s, s}\left(n_{2}^{2} \alpha^{2} q_{c}^{2} / 2 n_{1} P_{s}\right),(5)$ the financial-holding of raw material while in inventory, $h_{s, f_{1}}\left(n_{2}^{2} \alpha^{2} q_{c}^{2} /\right.$ $2 n_{1} P_{s}$ ), and (6) the financial-holding (losing opportunity) of items while in the vendor's inventory until it is withdrawn, $h_{s, f_{2}} n_{2}^{2} q_{c}^{2}\left(\left(\left(m_{1}+1\right) \alpha / 2 m_{1} P_{v}\right)-\left(\left(n_{1}-1\right) \alpha^{2} / 2 n_{1} P_{s}\right)\right)$. The total annual profit for the supplier is equal to the revenue it generates minus all costs per cycle (mentioned above) divided by the cycle time $\left(n_{2} q_{c} / D\right)$. The supplier's annual profit is found to be concave in $q_{c}$ since its second derivative is negative $\forall q_{c}>0$ and it is written as 


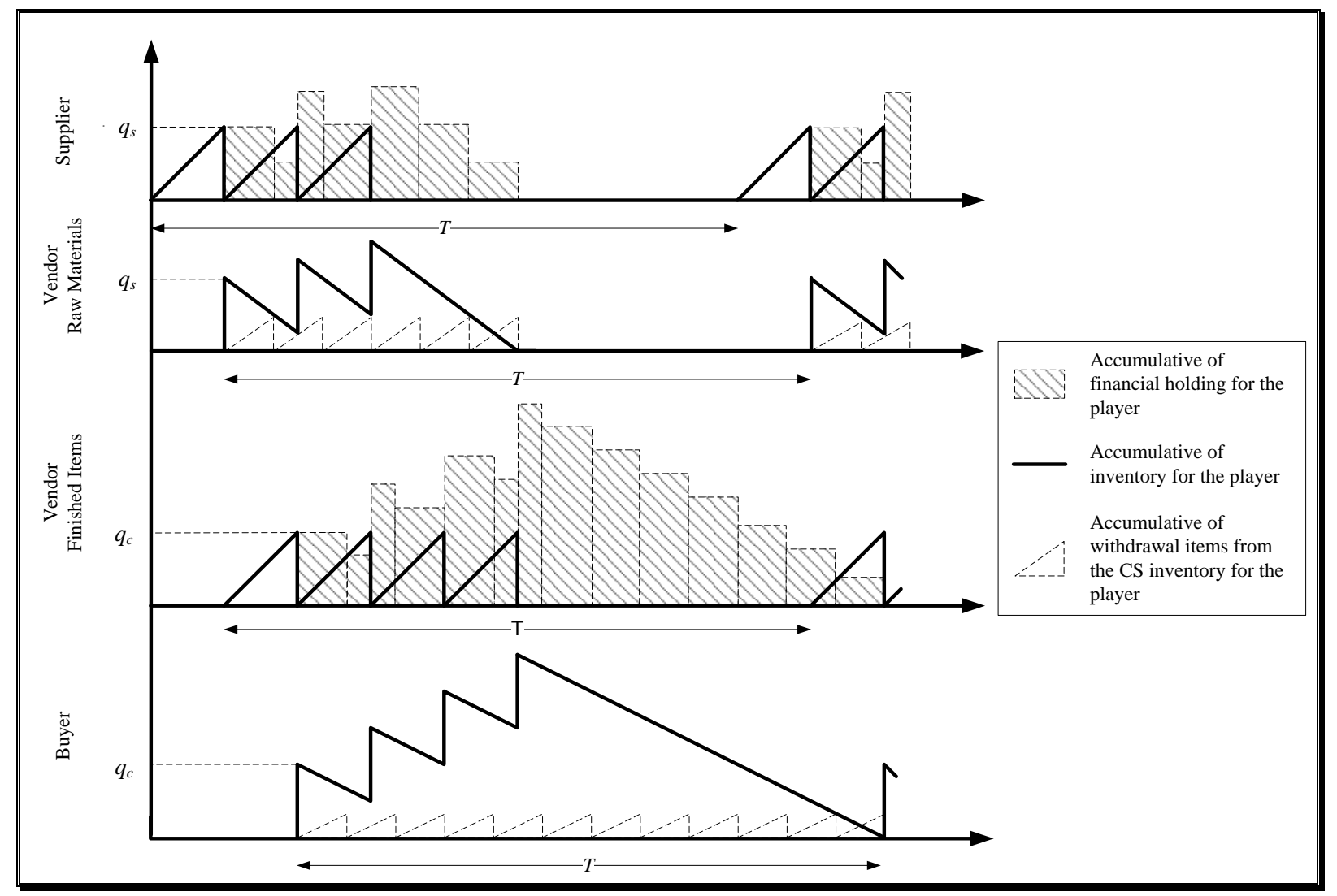

Figure 7-1. The behavior of the Consignment - Consignment (C-C) Scenario

$$
\begin{aligned}
T P_{C-C}^{s}=\left(\alpha c_{s}^{s}\right. & \left.-\gamma \alpha c_{s}^{p}-\alpha c_{s}^{p r}\right) D \\
& -\left(S_{s} \frac{D}{n_{2} q_{c}}+h_{s, f_{2}} n_{2} q_{c} D\left(\frac{\left(m_{1}+1\right) \alpha}{2 m_{1} P_{v}}-\frac{\left(n_{1}-1\right) \alpha^{2}}{2 n_{1} P_{s}}\right)\right. \\
& \left.+\left(h_{s, s}+h_{s, f_{1}}\right) \frac{n_{2} \alpha^{2} q_{c} D}{2 n_{1} P_{s}}\right)
\end{aligned}
$$

The vendor generates revenue in every cycle from selling its inventory of finished items to the buyer; i.e., $c_{v}^{s} n_{2} q_{c}$, and incurs the following costs per cycle: (1) raw material purchasing, $c_{s}^{S} n_{2} \alpha q_{c}$, (2) ordering, $n_{1} O_{v}$, (3) setup, $S_{v}$, (4) production, $c_{v}^{p r} n_{2} q_{c}$, (5) storage-holding of the raw material until it is used in the production, $h_{v, s}^{r}\left(n_{2} q_{c}^{2} / 2 P_{v}\right)\left(n_{2} \alpha-\left(n_{1}-1\right) n_{2} \alpha^{2} P_{v} / n_{1} P_{s}\right)$, (6) transaction per payment, $c_{v}^{t} m_{1}$, (7) the storage-holding of the finished items while in inventory, $h_{v, S}^{f}\left(n_{2} q_{c}^{2} / 2 P_{v}\right)$, and (8) the financial-holding of the finished items in its inventory, $h_{v, f_{1}}^{f}\left(n_{2} q_{c}^{2} / 2 P_{v}\right),(9)$ the financial-holding (losing opportunity) for an item in the buyer's inventory 
until it is withdrawn, $h_{v, f_{2}}^{f}\left(\left(\left(m_{2}+1\right) n_{2}^{2} q_{c}^{2} / 2 m_{2} D\right)-\left(n_{2} q_{c}^{2}\left(n_{2}-1\right) / 2 P_{v}\right)\right)$. The vendor's total annual profit is determined in a similar manner to Eq. (7-1). It is also concave in $q_{c}$ and it is given as

$$
\begin{aligned}
T P_{C-C}^{v}=\left(c_{v}^{s}\right. & \left.-\alpha c_{s}^{s}-c_{v}^{p r}\right) D-\left(S_{v}+n_{1} O_{v}+m_{1} c_{v}^{t}\right) \frac{D}{n_{2} q_{c}} \\
& -\left(h_{v, s}^{r} \frac{q_{c} D}{2 P_{v}}\left(n_{2} \alpha-\left(n_{1}-1\right) \frac{n_{2} \alpha^{2} P_{v}}{n_{1} P_{s}}\right)\right. \\
& \left.+h_{v, f_{2}}^{f}\left(\left(\frac{m_{2}+1}{2 m_{2}}\right) n_{2} q_{c}-\left(n_{2}-1\right) \frac{q_{c} D}{2 P_{v}}\right)+\left(h_{v, s}^{f}+h_{v, f_{1}}^{f}\right)\left(\frac{q_{c} D}{2 P_{v}}\right)\right)
\end{aligned}
$$

There is also a consignment agreement between the buyer and the vendor. The buyer stores the finished items at its warehouse and pays for what are withdrawn from inventory; i.e. sold. The buyer's revenue from sales and investment per cycle are $c_{b}^{S} n_{2} q_{c}$ and $c_{b}^{s} I_{b}\left(n_{2}^{2} q_{c}^{2} / 2 D m_{2}\right)$, respectively, who incurs the following costs: (1) purchasing, $c_{v}^{s} n_{2} q_{c}$, (2) ordering, $n_{2} O_{b}$, (3) transaction per payment, $c_{b}^{t} m_{2}$, and (4) storage-holding of finished items while in inventory, $h_{b, s}\left(n_{2} q_{c}^{2} / 2 D\left(n_{2}-\left(n_{2}-1\right) D / P_{v}\right)\right)$. The total annual profit for the buyer is determined in a similar manner to Eq. (7-1). The buyer's annual profit is also concave in $q_{c}$ and is given as

$$
\begin{gathered}
T P_{C-C}^{b}=\left(c_{b}^{s}-c_{v}^{S}\right) D+c_{b}^{S} I_{b}\left(\frac{n_{2} q_{c}}{2 m_{2}}\right)-\left(n_{2} O_{b}+m_{2} c_{b}^{t}\right) \frac{D}{n_{2} q_{c}} \\
-\frac{h_{b, s}}{2}\left(n_{2} q_{c}-\left(n_{2}-1\right) \frac{q_{c} D}{P_{v}}\right)
\end{gathered}
$$

Therefore, the total annual system profit for the C-C Scenario $\left(T P_{C-C}^{\text {system }}\right)$ is equal to the summation of the total annual profits of the supplier, the vendor and the buyer; i.e. $T P_{C-C}^{\text {system }}=$ $T P_{C-C}^{S}+T P_{C-C}^{v}+T P_{C-C}^{b}$, which is concave in $q_{c}$ (see Appendix 5 for proof) and whose optimum value that maximizes $T P_{C-C}^{\text {system }}$ is 


$$
\begin{aligned}
& q_{c}^{*} \\
& \sqrt{\frac{\left(S_{s}+S_{v}+n_{1} O_{v}+n_{2} O_{b}+m_{1} c_{v}^{t}+m_{2} c_{b}^{t}\right) \frac{D}{n_{2}}}{\left(\frac{\left(m_{1}+1\right) \alpha n_{2}}{m_{1}} h_{s, f_{2}}+n_{2} \alpha h_{v, s}^{r}+h_{v, s}^{f}+h_{v, f_{1}}^{f}-\left(n_{2}-1\right)\left(h_{v, f_{2}}^{f}+h_{b, s}\right)\right) \frac{D}{2 P_{v}}}}
\end{aligned}
$$

Since shortages are not allowed, then the optimum lot size $\left(n_{1}^{*} q_{s}^{*}\right)$ shipped to the vendor has to be enough to produce the required quantity of finished items; i.e. $n_{2}^{*} \alpha q_{c}^{*}$. The optimum size of each supplier's shipment $\left(q_{\mathrm{s}}^{*}\right)$ is given as

$$
q_{s}^{*}=\frac{n_{2}^{*} \alpha q_{c}^{*}}{n_{1}^{*}}, \text { where }\left\{\begin{array}{c}
n_{1}^{*}, n_{2}^{*} \text { and } \alpha \geq 1 \\
n_{2}^{*} \geq n_{1}^{*} \\
n_{1}^{*}, n_{2}^{*}, \text { and } \alpha \text { are integers }
\end{array}\right.
$$

\subsection{Scenario 2: No Consignment-Consignment (NC-C) Scenario:}

The second scenario assumes that there is no consignment agreement between the supplier and the vendor, but between the vendor and the buyer; NC-C Scenario. The behavior of inventory for the three players is shown in Figure 7-2 (next page). The same payment scheme as in the C-C Scenario is adopted between the vendor and the buyer, and the buyer's revenue from sales is invested until a payment is made.

The supplier's total revenue per cycle is $c_{s}^{s} n_{2} \alpha q_{c}$, who incurs the following costs: (1) raw material purchasing, $c_{s}^{p} n_{2} \gamma \alpha q_{c}$, (2) setup, $S_{s}$, (3) production, $c_{s}^{p r} n_{2} \alpha q_{c}$, (4) the storage-holding of raw material while in inventory, $h_{s, s}\left(n_{2}^{2} \alpha q_{c}^{2}\left(P_{S}-\alpha P_{v}\right) / 2 P_{S} P_{v}\right)$, and (5) the financial-holding of raw material while in inventory, $h_{s, f_{1}}\left(n_{2}^{2} \alpha q_{c}^{2}\left(P_{s}-\alpha P_{v}\right) / 2 P_{s} P_{v}\right)$. The supplier incurs no financialholding (losing opportunity) cost for the items stored at the vendor's side since the payment is made when the shipment is received by the vendor. The total annual profit for the supplier is determined in a similar manner to Eq. (7-1). The supplier's annual profit is also concave in $q_{c}$ and is written as 


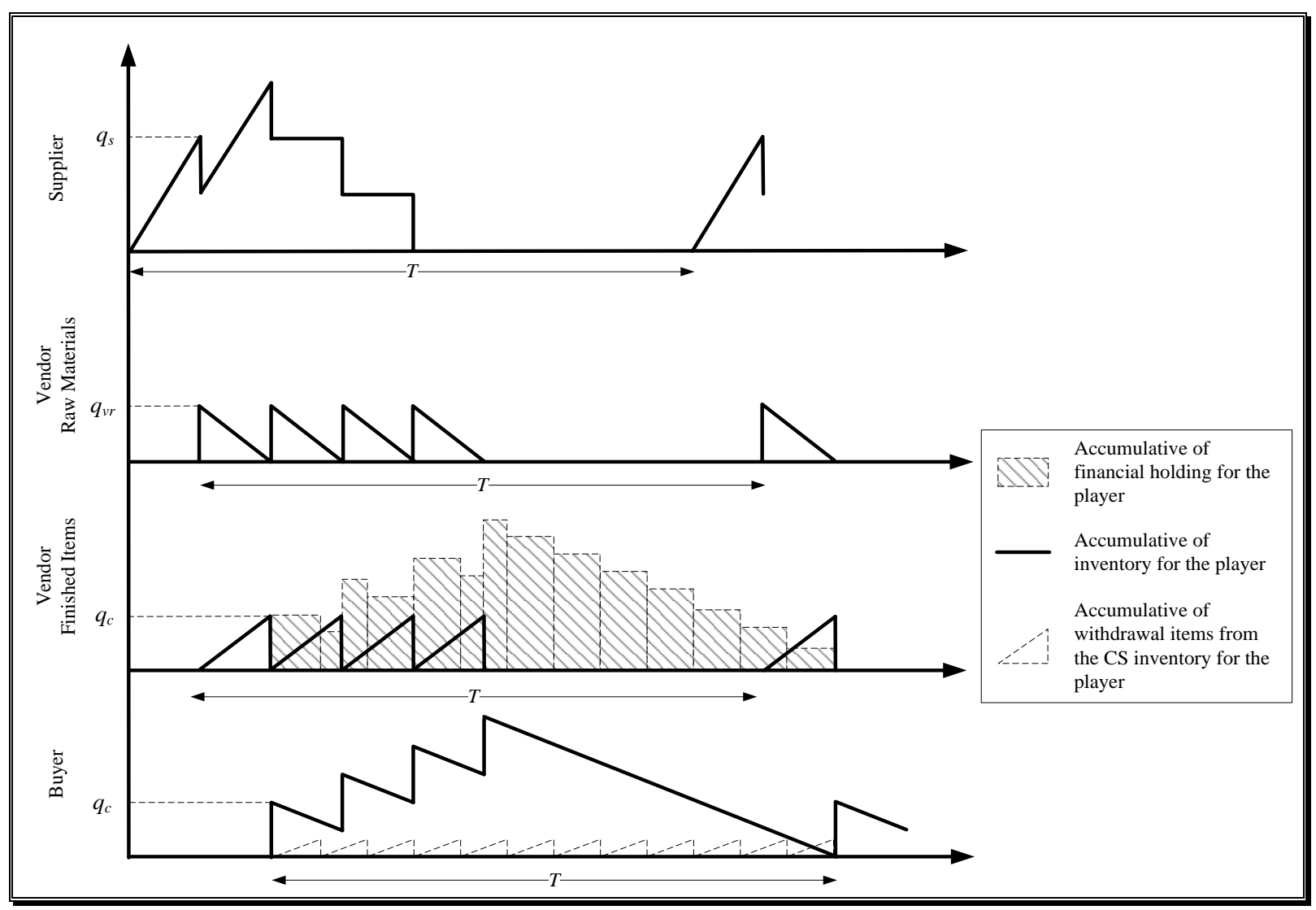

Figure 7-2. The behavior of the No Consignment - Consignment (NC-C) Scenario

$$
T P_{N C-C}^{s}=\left(\alpha c_{s}^{s}-\gamma \alpha c_{s}^{p}-\alpha c_{s}^{p r}\right) D-\left(S_{s} \frac{D}{n_{2} q_{c}}+\left(h_{s, s}+h_{s, f_{1}}\right) \frac{n_{2} \alpha q_{c} D}{2 P_{s} P_{v}}\left(P_{s}-\alpha P_{v}\right)\right) \quad \text { 7-6 }
$$

The vendor's revenue per cycle for the NC-C Scenario is $c_{v}^{S} n_{2} q_{c}$, who incurs the following costs: (1) raw material purchasing, $c_{s}^{s} n_{2} \alpha q_{c}$, (2) ordering, $n_{1} O_{v}$, (3) setup, $S_{v}$, (4) production, $c_{v}^{p r} n_{2} q_{c}$, (5) the storage-holding of the raw material while in inventory, $h_{v, s}^{r}\left(\alpha n_{2} q_{v}^{2} / 2 P_{v}\right)$, (6) the financialholding of raw material until it is used in production, $h_{v, f}^{r}\left(\alpha n_{2} q_{v}^{2} / 2 P_{v}\right)$, (7) transaction of payments, $c_{v}^{t} n_{1}$, (8) the storage-holding of the finished items while in inventory, $h_{v, s}^{f}\left(n_{2} q_{c}^{2} / 2 P_{v}\right)$, (9) the financial-holding of the finished items while in inventory, $h_{v, f_{1}}^{f}\left(n_{2} q_{c}^{2} / 2 P_{v}\right)$, and (10) the financial-holding (losing opportunity) of sold items in the buyer's inventory until the latter pays for the withdrawn items, $h_{v, f_{2}}^{f} q_{c}^{2}\left(\left(m_{2}+1\right) n_{2}^{2} / 2 m_{2} D-\left(n_{2}-1\right) n_{2} / 2 P_{v}\right)$. The vendor's annual total profit $\left(T P_{N C-C}^{v}\right)$ is determined in a similar manner to Eq. (7-1). The vendor's annual profit is concave in $q_{c}$ and is given as 


$$
\begin{aligned}
T P_{N C-C}^{v}=\left(c_{v}^{s}\right. & \left.-\alpha c_{s}^{s}-c_{v}^{p r}\right) D-\left(S_{v}+n_{1} O_{v}+n_{1} c_{v}^{t}\right) \frac{D}{n_{2} q_{c}} \\
& -\left(\left(h_{v, s}^{r}+h_{v, f}^{r}\right) \frac{\alpha D}{2 P_{v}} q_{c}+h_{v, f_{2}}^{f}\left(\left(\frac{m_{2}+1}{2 m_{2}}\right) n_{2} q_{c}-\left(n_{2}-1\right) \frac{q_{c} D}{2 P_{v}}\right)\right. \\
& \left.+\left(h_{v, s}^{f}+h_{v, f_{1}}^{f}\right) \frac{q_{c} D}{2 P_{v}}\right)
\end{aligned}
$$

The buyer's total revenue from sales and investment per cycle is $c_{b}^{s} n_{2} q_{c}$ and $c_{b}^{s} I_{b}\left(n_{2}^{2} q_{c}^{2} / 2 D m_{2}\right)$, respectively, who incurs the following costs: (1) purchasing, $c_{v}^{s} n_{2} q_{c}$, (2) ordering, $n_{2} O_{b}$, (3) transaction of payments, $c_{b}^{t} m_{2}$, and (4) the storage-holding for finished items while in inventory, $h_{b}\left(n_{2} q_{c}^{2} / 2 D\left(n_{2}-\left(n_{2}-1\right) D / P_{v}\right)\right)$. The total annual profit for the buyer is determined in a similar manner to Eq. (7-1). The buyer's annual profit is concave in $q_{c}$ and is given as

$$
\begin{gathered}
T P_{N C-C}^{b}=\left(c_{b}^{s}-c_{v}^{S}\right) D+c_{b}^{S} I_{b}\left(\frac{n_{2} q_{c}}{2 m_{2}}\right)-\left(n_{2} O_{b}+m_{2} c_{b}^{t}\right) \frac{D}{n_{2} q_{c}} \\
-\frac{h_{b, s}}{2}\left(n_{2} q_{c}-\left(n_{2}-1\right) \frac{q_{c} D}{P_{v}}\right)
\end{gathered}
$$

The total annual system profit for the NC-C Scenario is the summation of Eqs. (7-6), (7-7) and (7-8) and is concave in $q_{c}$ (see Appendix 5 for proof) and whose optimum value that maximizes $T P_{N C-C}^{\text {system }}$ is given as

$$
q_{c}^{*}=\sqrt{\frac{\left(S_{s}+S_{v}+n_{1} O_{v}+n_{2} O_{b}+n_{1} c_{v}^{t}+m_{2} c_{b}^{t}\right) \frac{D}{n_{2}}}{\left(n_{2} \alpha\left(h_{s, s}+h_{s, f_{1}}\right)+\alpha\left(h_{v, s}^{r}+h_{v, f}^{r}\right)+h_{v, s}^{f}+h_{v, f_{1}}^{f}-\left(n_{2}-1\right)\left(h_{v, f_{2}}^{f}+h_{b, s}\right)\right) \frac{D}{2 P_{v}}}} 7-9
$$

The optimum lot size $\left(n_{1}^{*} q_{v r}^{*}\right)$ that is sent to the vendor has to be sufficient for producing a total of $n_{2}^{*} \alpha q_{c}^{*}$ units, where $\left(q_{v r}^{*}\right)$ is calculated from Eq. (7-5) after replacing $q_{s}^{*}$ with $q_{v r}^{*}$. 


\subsection{Scenario 3: Consignment-No Consignment (C-NC) Scenario:}

This scenario considers a consignment agreement between the supplier and the vendor, but not between the vendor and the buyer who adopt a traditional coordination policy. The behavior of inventory for the three players for this scenario, C-NC, is represented in Figure 7-3. An equal payment scheme is adopted between the vendor and the supplier.

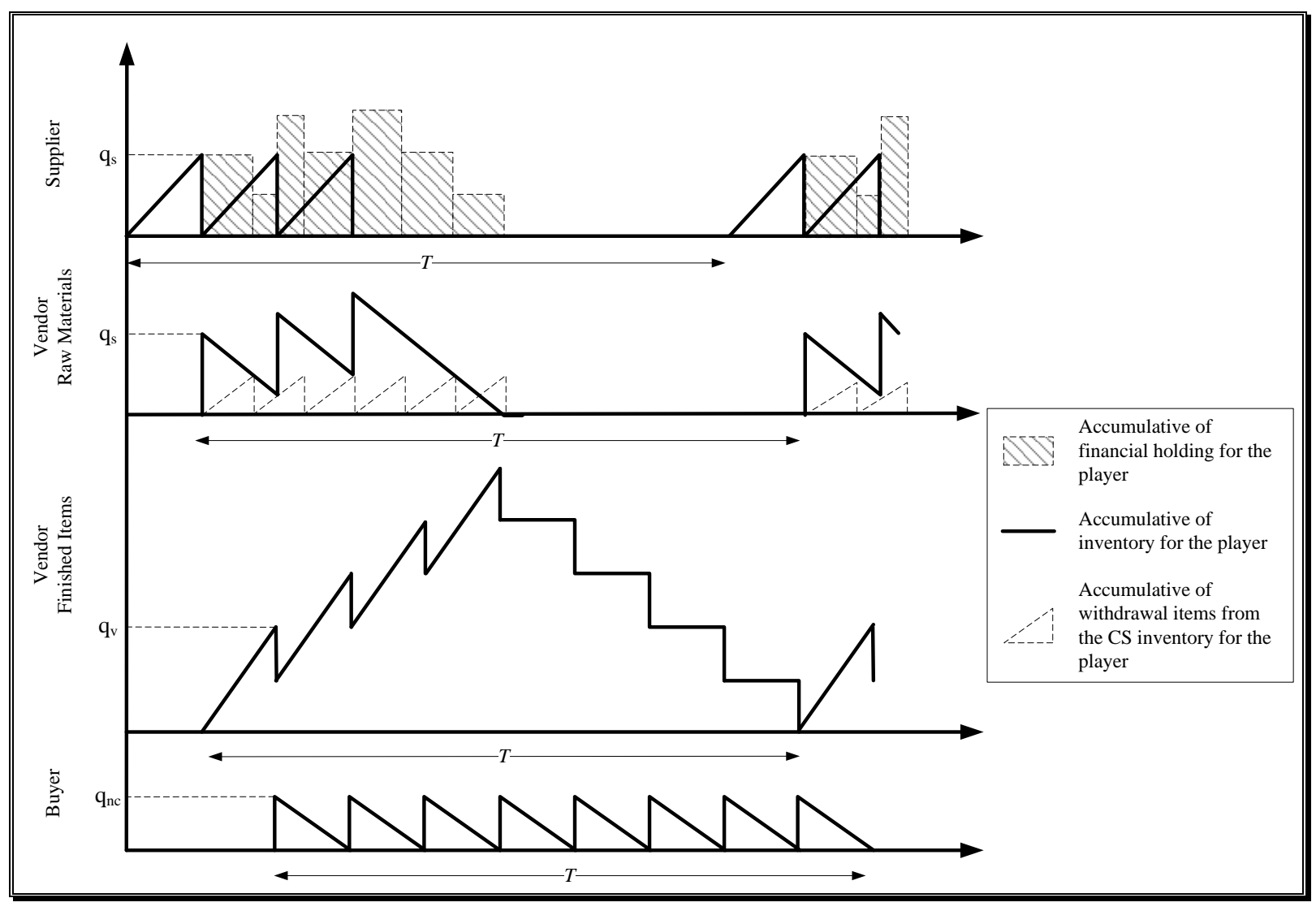

Figure 7-3. The behavior of the Consignment - No Consignment (C-NC) Scenario

The supplier generates revenue of $c_{s}^{s} n_{2} \alpha q_{n c}$ per cycle, and incurs the following costs: (1) raw material purchasing, $c_{s}^{p} n_{2} \gamma \alpha q_{n c}$, (2) setup, $S_{s}$, (3) production, $c_{s}^{p r} n_{2} \alpha q_{n c}$, (4) the storage-holding of finished raw material while in inventory, $h_{s, s}\left(n_{2}^{2} \alpha^{2} q_{n c}^{2} / 2 n_{1} P_{s}\right)$, (5) the financial-holding of finished raw material products while in inventory, $h_{s, f_{1}}\left(n_{2}^{2} \alpha^{2} q_{n c}^{2} / 2 n_{1} P_{s}\right)$, and (6) the financialholding (losing opportunity) of the items in the vendor's inventory until they are withdrawn, $h_{s, f_{2}} n_{2}^{2} q_{n c}^{2}\left(\left(\left(m_{1}+1\right) \alpha / 2 m_{1} P_{v}\right)-\left(\left(n_{1}-1\right) \alpha^{2} / 2 n_{1} P_{s}\right)\right)$. The total annual profit for the supplier 
is determined in a similar manner to Eq. (7-1). The supplier's annual profit is concave in $q_{n c}$ and is given as

$$
\begin{aligned}
& T P_{C-N C}^{s}=\left(\alpha c_{s}^{s}-\gamma \alpha c_{s}^{p}-\alpha c_{s}^{p r}\right) D \\
&-\left(S_{s} \frac{D}{n_{2} q_{n c}}+h_{s, f_{2}} n_{2} q_{n c} D\left(\frac{\left(m_{1}+1\right) \alpha}{2 m_{1} P_{v}}-\frac{\left(n_{1}-1\right) \alpha^{2}}{2 n_{1} P_{s}}\right)\right. \\
&\left.+\left(h_{s, s}+h_{s, f_{1}}\right)\left(\frac{n_{2} \alpha^{2} q_{n c} D}{2 n_{1} P_{s}}\right)\right)
\end{aligned}
$$

The vendor generates revenue of $c_{v}^{s} n_{2} q_{n c}$ per cycle and incurs the following costs: (1) raw material purchasing, $c_{s}^{s} n_{2} \alpha q_{n c}$, (2) ordering, $n_{1} O_{v}$, (3) setup, $S_{v}$, (4) production, $c_{v}^{p r} n_{2} q_{n c}$, (5) the storage-holding of raw material while in inventory, $h_{v, S}^{r}\left(n_{2} q_{n c}^{2} / 2 P_{v}\right)\left(n_{2} \alpha-\left(n_{1}-1\right) n_{2} \alpha^{2} P_{v} /\right.$ $\left.n_{1} P_{s}\right)$, (6) transaction of payments, $c_{v}^{t} m_{1}$, (7) the storage-holding of finished items while in inventory, $h_{v, s}^{f}\left(n_{2}^{2} q_{n c}^{2}\left(P_{v}-D\right) / 2 D P_{v}\right)$, and (8) the financial-holding of finished items while in inventory, $h_{v, f_{1}}^{f}\left(n_{2}^{2} q_{n c}^{2}\left(P_{v}-D\right) / 2 D P_{v}\right)$. In this scenario, the vendor incurs no financial-holding (losing opportunity) cost for items stored at the buyer's facility as the payment is made upon receiving a shipment. The vendor's annual total profit is determined in a similar manner to Eq. (7-1). The vendor's annual profit is concave in $q_{n c}$ and is given as

$$
\begin{aligned}
T P_{C-N C}^{v}=\left(c_{v}^{s}\right. & \left.-\alpha c_{s}^{s}-c_{v}^{p r}\right) D-\left(S_{v}+n_{1} O_{v}+m_{1} c_{v}^{t}\right) \frac{D}{n_{2} q_{n c}} \\
& -\left(h_{v, s}^{r} \frac{q_{n c} D}{2 P_{v}}\left(n_{2} \alpha-\left(n_{1}-1\right) \frac{n_{2} \alpha^{2} P_{v}}{n_{1} P_{s}}\right)\right. \\
& \left.+\left(h_{v, s}^{f}+h_{v, f_{1}}^{f}\right) \frac{\left(P_{v}-D\right) n_{2} q_{n c}}{2 P_{v}}\right)
\end{aligned}
$$

The buyer generates revenue of $c_{b}^{S} n_{2} q_{n c}$ per cycle, and incurs the following costs: (1) ordering, $n_{2} O_{b}$, (2) transaction of payments, $c_{b}^{t} n_{2}$, (3) purchasing, $c_{v}^{s} n_{2} q_{n c}$, (4) the storage-holding of the finished items while in inventory, $h_{b, s}\left(n_{2} q_{n c}^{2} / 2 D\right)$, and (5) the financial-holding of the finished items while in inventory, $h_{b, f}\left(n_{2} q_{n c}^{2} / 2 D\right)$. The buyer's total annual profit is determined in a similar manner to Eq. (7-1). The buyer's annual profit is concave in $q_{n c}$ and is given as 


$$
T P_{C-N C}^{b}=\left(c_{b}^{s}-c_{v}^{s}\right) D-\left(O_{b}+c_{b}^{t}\right) \frac{D}{q_{n c}}-\left(h_{b, s}+h_{b, f}\right) \frac{q_{n c}}{2}
$$

The system total annual profit in this scenario, $\left(T P_{C-N C}^{\text {system }}\right)$, is the summation of Eqs. (7-10), (7-11) and (7-12), and is concave in $q_{n c}$ (see Appendix 5 for proof), whose optimum value is given as

$$
q_{n c}^{*}=\sqrt{\frac{\left(S_{s}+S_{v}+n_{1} O_{v}+n_{2} O_{b}+m_{1} c_{v}^{t}+n_{2} c_{b}^{t}\right) \frac{D}{n_{2}}}{\left(\frac{\left(m_{1}+1\right) \alpha n_{2}}{m_{1}} h_{s, f_{2}}+n_{2}\left(\alpha h_{v, s}^{r}-h_{v, S}^{f}-h_{v, f_{1}}^{f}\right)\right) \frac{D}{2 P_{v}}}}
$$

The optimum batch quantity $\left(q_{s}^{*}\right)$ that is sent by the supplier to the vendor is found from Eq. (7-5) after replacing $q_{c}^{*}$ with $q_{n c}^{*}$.

\subsection{Scenario 4: No Consignment-No Consignment (NC-NC) Scenario:}

This scenario considers traditional supply chain coordination, no consignment, among the players. For this scenario, the vendor (retailer) pays the supplier (vendor) upon receiving a shipment of raw material (finished items). As a result, there is no financial-holding (losing opportunity) cost that relates to payments, but it is incurred for items in inventory until a shipment is made. The behavior of inventory for the three players in this scenario, NC-NC, is shown in Figure 7-4 (next page).

The supplier's revenue is $c_{s}^{S} n_{2} \alpha q_{n c}$ per cycle, where it incurs the following cost: (1) purchasing raw material, $c_{s}^{p} n_{2} \gamma \alpha q_{n c}$, (2) setup, $S_{s}$, (3) production, $c_{s}^{p r} n_{2} \alpha q_{n c}$, (4) the storage-holding of finished raw material while in inventory, $h_{s, s}\left(n_{2}^{2} \alpha q_{n c}^{2}\left(P_{s}-\alpha P_{v}\right) / 2 P_{v} P_{s}\right)$, and (5) the financialholding of finished raw material while in inventory, $h_{s, f_{1}}\left(n_{2}^{2} \alpha q_{n c}^{2}\left(P_{s}-\alpha P_{v}\right) / 2 P_{v} P_{s}\right)$. The supplier's total annual profit is determined in a similar manner to Eq. (7-1). The supplier's annual profit is concave in $q_{n c}$ and is given as

$$
T P_{N C-N C}^{S}=\left(\alpha c_{s}^{S}-\gamma \alpha c_{s}^{p}-\alpha c_{s}^{p r}\right) D-\left(S_{s} \frac{D}{n_{2} q_{n c}}+\left(h_{s, s}+h_{s, f_{1}}\right) \frac{n_{2} \alpha q_{n c} D\left(P_{s}-\alpha P_{v}\right)}{2 P_{v} P_{s}}\right) 7-14
$$




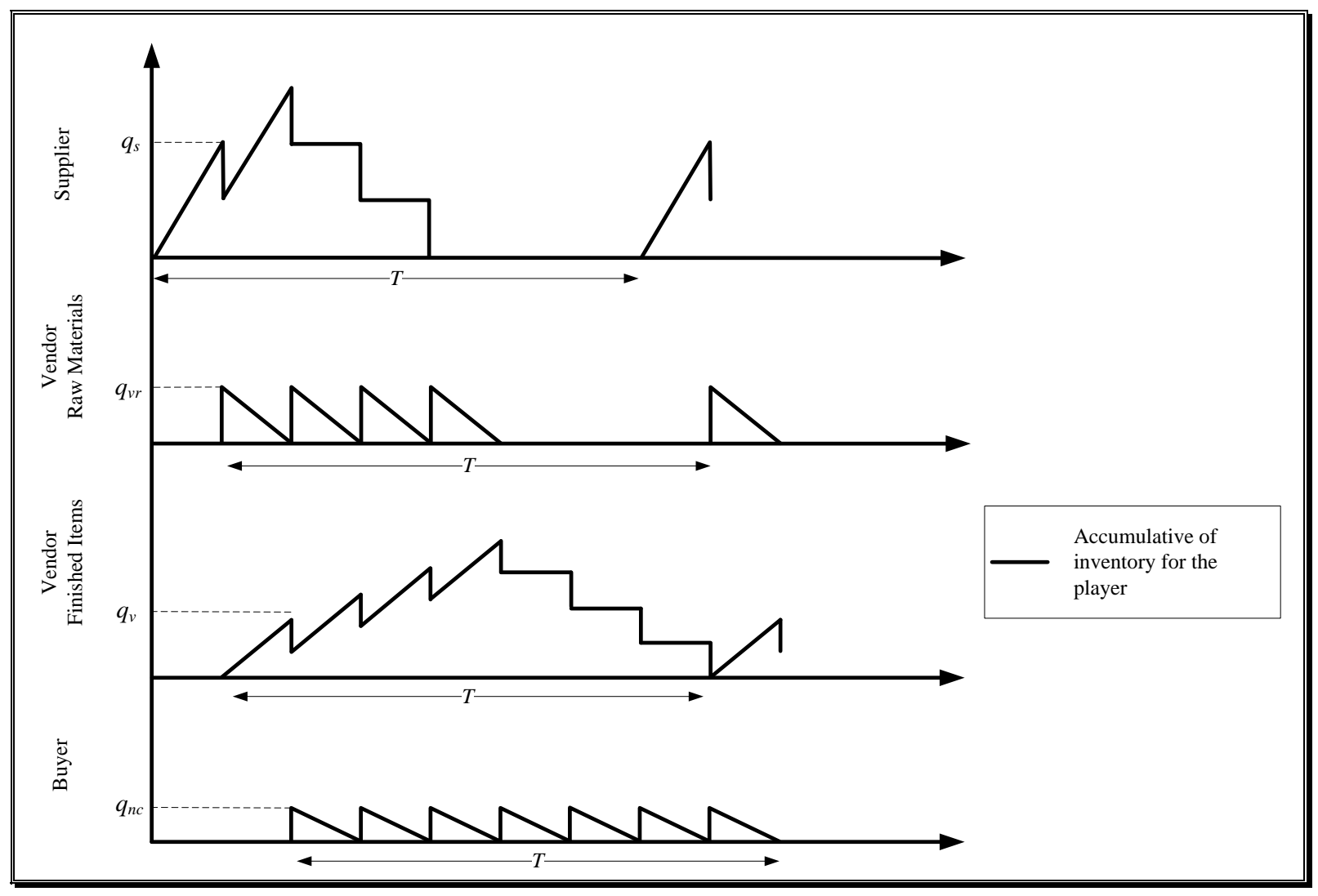

Figure 7-4. The behavior of the No Consignment - No Consignment (NC-NC) Scenario

The vendor generates revenue of $c_{v}^{s} n_{2} q_{n c}$ per cycle, and incurs the following costs: (1) raw material purchasing, $c_{s}^{s} n_{2} \alpha q_{n c}$, (2) ordering, $n_{1} O_{v}$, (3) setup, $S_{v}$, (4) production, $c_{v}^{p r} n_{2} q_{n c}$, (5) the storage-holding of the raw material while in inventory, $h_{v, s}^{r}\left(n_{2}^{2} \alpha q_{n c}^{2} / 2 n_{1} P_{v}\right)$, (6) the financialholding of the raw material until it is used in production, $h_{v, f}^{r}\left(n_{2}^{2} \alpha q_{n c}^{2} / 2 n_{1} P_{v}\right)$, (7) transaction of payments, $c_{v}^{t} n_{1}$, (8) the storage-holding of the finished items while in inventory, $h_{v, S}^{f}\left(n_{2}^{2} q_{n c}^{2}\left(P_{v}-\right.\right.$ $\left.D) / 2 D P_{v}\right)$, and (9) the financial-holding of the finished items while in inventory, $h_{v, f_{1}}^{f}\left(n_{2}^{2} q_{n c}^{2}\left(P_{v}-\right.\right.$ $\left.D) / 2 D P_{v}\right)$. The vendor's annual total profit $\left(T P_{N C-N C}^{v}\right)$ is determined in a similar manner to Eq. (7-1). The vendor's annual profit is concave in $q_{n c}$ and is given as

$$
\begin{aligned}
T P_{N C-N C}^{v}=\left(c_{v}^{s}-\alpha c_{s}^{s}-c_{v}^{p r}\right) D-\left(S_{v}+n_{1} O_{v}+n_{1} c_{v}^{t}\right) \frac{D}{n_{2} q_{n c}} \\
-\left(\left(h_{v, s}^{r}+h_{v, f}^{r}\right) \frac{n_{2} \alpha q_{n c} D}{2 n_{1} P_{v}}+\left(h_{v, s}^{f}+h_{v, f_{1}}^{f}\right) \frac{n_{2} q_{n c}}{2 P_{v}}\left(P_{v}-D\right)\right)
\end{aligned}
$$


The buyer generates revenue of $c_{b}^{S} n_{2} q_{n c}$ per cycle, and incurs the following costs: (1) ordering, $n_{2} O_{b}$, (2) transaction of payments, $c_{b}^{t} n_{2}$, (3) purchasing, $c_{v}^{s} n_{2} q_{n c}$, (4) the storage-holding of finished items, $h_{b, s}\left(n_{2} q_{n c}^{2} / 2 D\right)$, and (5) the financial-holding of finished items while in inventory, $h_{b, f}\left(n_{2} q_{n c}^{2} / 2 D\right)$. The buyer total annual profit is determined in a similar manner to Eq. (7-1). The buyer's annual profit is concave in $q_{n c}$ and is given as

$$
T P_{N C-N C}^{b}=\left(c_{b}^{s}-c_{v}^{s}\right) D-\left(O_{b}+c_{b}^{t}\right) \frac{D}{q_{n c}}-\left(h_{b, s}+h_{b, f}\right) \frac{q_{n c}}{2}
$$

The system total profit for the NC-NC Scenario $\left(T P_{N C-N C}^{\text {system }}\right)$ is equal to the summation of Eqs. (7-14), (7-15) and (7-16), and concave in $q_{n c}$ (see Appendix 5 for proof), whose optimum value is given as

$$
\begin{aligned}
& q_{n c}^{*}
\end{aligned}
$$

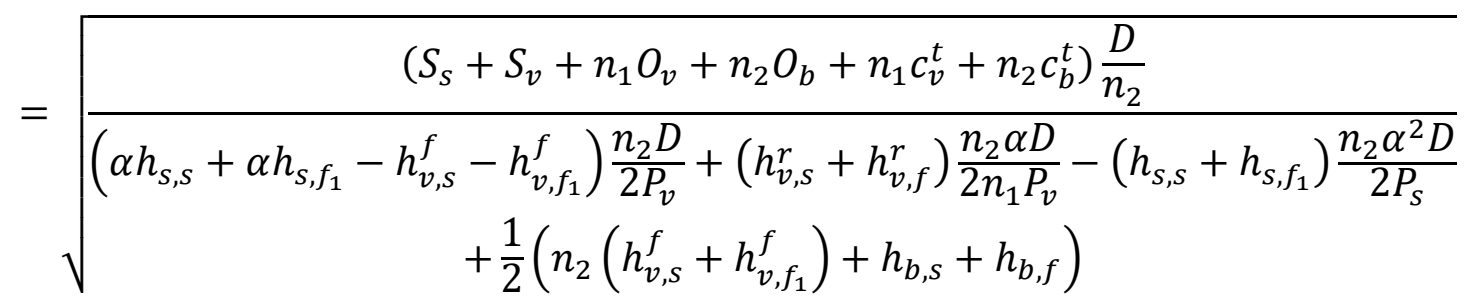

Eq. (7-5) is used to find the optimum quantity of the raw material that is shipped by the supplier to the vendor $\left(q_{v r}^{*}\right)$ after replacing $\left(q_{s}^{*}\right)$ with $\left(q_{v r}^{*}\right)$ and $\left(q_{c}^{*}\right)$ with $\left(q_{n c}^{*}\right)$.

\subsection{Solution Procedure}

The optimal solutions $\left(n_{1}^{*}, n_{2}^{*}, m_{1}^{*}\right.$ and $\left.m_{2}^{*}\right)$ for the developed models/scenarios can be found by using a nested loop search written with Visual Basic for Applications (VBA) codes following the steps described below, which are similar to those described in Jaber and Goyal (2008) and Chapter 5.

The steps are as follow and they are repeated for all scenarios:

Step 1-

a. Input the given input parameters for the supplier, the vendor and the buyer.

Step 2- 
a. Let $n_{1}, n_{2}, m_{1}$ and $m_{2}$ equal to $n_{1 x}, n_{2 x}, m_{1 x}$ and $m_{2 x}$ where $x$ represents the value of the decision variables and $x=1,2, \ldots X$.

b. Set $n_{11}, n_{21}, m_{11}$ and $m_{21}=1$.

c. Calculate $q_{C}\left(n_{11}, n_{21}, m_{11}, m_{21}\right)$ and $T P_{i}^{\text {system }}\left(n_{11}, n_{21}, m_{11}, m_{21}, q_{c}\left(n_{11}, n_{21}, m_{11}, m_{21}\right)\right)$.

d. Set the value of $T P_{i}^{\text {system }}\left(n_{11}, n_{21}, m_{11}, m_{21}, q_{c}\left(n_{11}, n_{21}, m_{11}, m_{21}\right)\right)=$ Value A.

e. Increase $x$ for $m_{2 x}$ by 1 .

f. Calculate $q_{C}\left(n_{11}, n_{21}, m_{11}, m_{2 x}\right)$ and $T P_{i}^{\text {system }}\left(n_{11}, n_{21}, m_{11}, m_{2 x}, q_{C}\left(n_{11}, n_{21}, m_{11}, m_{2 x}\right)\right)$.

g. Set the value of $T P_{i}^{\text {system }}\left(n_{11}, n_{21}, m_{11}, m_{2 x}, q_{C}\left(n_{11}, n_{21}, m_{11}, m_{2 x}\right)\right)=$ Value B.

h. Compare Value A with Value B.

i. If Value A > Value B, then Go to Step 3.

ii. Otherwise, set Value $B=$ Value $A$ and Go back to Step 2.e.

Step 3-

a. Increase $m_{1 x}$ by 1 . Note that $m_{2 x} \geq m_{1 x}$. Calculate $q_{C}\left(n_{11}, n_{21}, m_{1 x}, m_{2 x}\right)$ and $T P_{i}^{\text {system }}\left(n_{11}, n_{21}, m_{1 x}, m_{2 x}, q_{c}\left(n_{11}, n_{21}, m_{1 x}, m_{2 x}\right)\right)=$ Value B.

b. Compare Value B in this step with Value A from Step 2.

i. If Value $\mathrm{B}>$ Value $\mathrm{A}$ set Value $\mathrm{B}=$ Value $\mathrm{A}$ and start searching for $m_{2 x}$ similar to what have been done in Step 2.

ii. Otherwise, go to Step 4.

Step 4-

a. Increase $n_{2 x}$ by 1 . Calculate $q_{C}\left(n_{11}, n_{2 x}, m_{1 x}, m_{2 x}\right)$ and $T P_{i}^{\text {system }}\left(n_{11}, n_{2 x}, m_{1 x}, m_{2 x}, q_{c}\left(n_{11}, n_{2 x}, m_{1 x}, m_{2 x}\right)\right)=$ Value B. 
b. Compare Value B in this step with Value A from previous steps.

i. If Value B > Value A set Value B = Value A and start searching for $m_{1 x}$ and $m_{2 x}$ similar to what have been done in Steps 2 and 3.

ii. Otherwise, go to Step 5.

Step 5-

a. Increase $n_{1 x}$ by 1 . Note that $n_{2} \geq n_{1}$, therefore, calculate $q_{C}\left(n_{1 x}, n_{2 x}, m_{1 x}, m_{2 x}\right)$ and $T P_{i}^{\text {system }}\left(n_{1 x}, n_{2 x}, m_{1 x}, m_{2 x}, q_{c}\left(n_{1 x}, n_{2 x}, m_{1 x}, m_{2 x}\right)\right)=$ Value B.

b. Compare Value B in this step with Value A from previous steps.

i. If Value B > Value A, set Value B = Value A and start searching for $m_{1 x}, m_{2 x}$ and $n_{2 x}$ similar to what have been done in Steps 2, 3 and 4 .

ii. Otherwise, Value A is optimal solution and the values for its decision variables are optimal.

Repeat the steps above and stop when you find the maximum $T P_{i}^{\text {system }}$. The values for $n_{1}, n_{2}, m_{1}$, and $m_{2}$ that return the maximum $T P_{i}^{\text {system }}$ are the optimal solutions $\left(n_{1}^{*}, n_{2}^{*}, m_{1}^{*}, m_{2}^{*}\right)$ which its optimal quantity is $q_{i}^{*}\left(n_{1}^{*}, n_{2}^{*}, m_{1}^{*}, m_{2}^{*}\right)$ where $i$ is for Scenarios 1,2, 3 and 4 . The optimal solutions for the decision variables are also used to find the total revenues, costs and profits for each player if needed.

\subsection{Numerical Example}

A numerical example with nine different cases is presented to compare between the developed scenarios. The values of some of the parameters were taken from the literature (Khouja, 2003; Jaber and Goyal, 2008; Battini et al., 2010b) and the others were set within reason or calculated. For example, the profit margin for each player (supplier, vendor, and buyer) is set at $40 \%$. The studies in the literature showed that the storage-holding cost is the component that impacts the decision of whether to follow a CS policy or a traditional policy. This example extensively investigates the four developed scenarios/models by considering nine cases for different combinations of storage-holding costs. Table 7-2 (next page) lists the parameters and their values where Table 7-3 (next page) lists the storage-holding costs for the nine cases. 
Table 7-2. Parameter values for the numerical example

\begin{tabular}{|c|c|c|c|c|c|}
\hline & $\begin{array}{c}\text { Supplier's parameters } \\
\text { values }\end{array}$ & & $\begin{array}{c}\text { Vendor's parameters } \\
\text { values }\end{array}$ & & $\begin{array}{c}\text { Buyer's parameters } \\
\text { values }\end{array}$ \\
\hline$P_{S}$ & $399,000.00$ (units/year) & $P_{v}$ & $140,000.00$ (units/year) & $D$ & $50,000.00$ (units/year) \\
\hline$\gamma$ & 1 & $\alpha$ & 1 & $O_{b}$ & 50.0 (\$/order) \\
\hline$S_{S}$ & 400 (\$/set-up) & $S_{v}$ & 200 (\$/set-up) & $c_{b}^{S}$ & $1.4 c_{v}^{S}=4.23$ (\$/unit) \\
\hline$c_{s}^{p r}$ & 0.40 (\$/unit) & $O_{v}$ & 40.0 (\$/order) & $h_{b, f}$ & $\begin{array}{l}I_{b} c_{v}^{S}=0.3 \\
\text { (\$/unit/year) }\end{array}$ \\
\hline$c_{s}^{p}$ & 0.50 (\$/unit) & $c_{v}^{p r}$ & 0.90 (\$/unit) & $c_{b}^{t}$ & $0.50(\$ /$ transaction $)$ \\
\hline$c_{S}^{S}$ & $\begin{array}{l}1.4\left(c_{s}^{p r}+c_{s}^{p}\right)=1.26 \\
(\$ / \text { unit })\end{array}$ & $c_{v}^{S}$ & $\begin{array}{l}1.4\left(c_{s}^{s}+c_{v}^{p r}\right)=3.02 \\
(\$ / \text { unit })\end{array}$ & $I_{b}$ & 10 (\%/year) \\
\hline$I_{S}$ & 10 (\%/year) & $I_{v}$ & 10 (\%/year) & & \\
\hline$h_{s, f_{1}}$ & $I_{s} c_{s}^{p r}=0.04(\$ /$ unit/year $)$ & $h_{v, f}^{r}$ & $I_{v} c_{S}^{S}=0.13(\$ /$ unit/year $)$ & & \\
\hline \multirow[t]{3}{*}{$h_{s, f_{2}}$} & $I_{s} c_{S}^{S}=0.13(\$ /$ unit/year $)$ & $h_{v, f_{1}}^{f}$ & $I_{v} c_{v}^{p r}=0.09(\$ /$ unit/year $)$ & & \\
\hline & & $h_{v, f_{2}}^{f}$ & $I_{v} c_{v}^{S}=0.3(\$ /$ unit/year $)$ & & \\
\hline & & $c_{v}^{t}$ & $0.50(\$ /$ transaction $)$ & & \\
\hline
\end{tabular}

Table 7-3. Storage-holding costs values for the raw materials and the finished items for the nine cases for each player

\begin{tabular}{|c|c|c|c|c|c|c|c|}
\hline \multirow{2}{*}{ Case } & \multirow{2}{*}{\multicolumn{3}{|c|}{ Conditions }} & \multicolumn{2}{|c|}{$\begin{array}{l}\text { The values of the raw } \\
\text { materials storage-holding cost }\end{array}$} & \multicolumn{2}{|c|}{$\begin{array}{l}\text { The values of the finished } \\
\text { items storage-holding cost }\end{array}$} \\
\hline & & & & $h_{s, s}$ & $h_{v, s}^{r}$ & $h_{v, S}^{f}$ & $h_{b, s}$ \\
\hline 1 & $h_{s, s}>h_{v, s}^{r}$ & and & $h_{v, s}^{f}>h_{b, s}$ & 0.3 & 0.15 & 0.7 & 0.5 \\
\hline 2 & $h_{s, s}=h_{v, s}^{r}$ & and & $h_{v, s}^{f}>h_{b, s}$ & 0.15 & 0.15 & 0.7 & 0.5 \\
\hline 3 & $h_{s, s}<h_{v, s}^{r}$ & and & $h_{v, s}^{f}>h_{b, s}$ & 0.15 & 0.3 & 0.7 & 0.5 \\
\hline 4 & $h_{s, s}>h_{v, s}^{r}$ & and & $h_{v, s}^{f}=h_{b, s}$ & 0.3 & 0.15 & 0.5 & 0.5 \\
\hline 5 & $h_{s, s}=h_{v, s}^{r}$ & and & $h_{v, s}^{f}=h_{b, s}$ & 0.15 & 0.15 & 0.5 & 0.5 \\
\hline 6 & $h_{s, s}<h_{v, s}^{r}$ & and & $h_{v, s}^{f}=h_{b, s}$ & 0.15 & 0.3 & 0.5 & 0.5 \\
\hline 7 & $h_{s, s}>h_{v, s}^{r}$ & and & $h_{v, s}^{f}<h_{b, s}$ & 0.3 & 0.15 & 0.5 & 0.7 \\
\hline 8 & $h_{s, s}=h_{v, s}^{r}$ & and & $h_{v, s}^{f}<h_{b, s}$ & 0.15 & 0.15 & 0.5 & 0.7 \\
\hline 9 & $h_{s, s}<h_{v, s}^{r}$ & and & $h_{v, s}^{f}<h_{b, s}$ & 0.15 & 0.3 & 0.5 & 0.7 \\
\hline
\end{tabular}

Table 7-4 (next page) represents an example of the manual search for the optimal solution of the C-C Scenario (Case 1) using the input parameters in Table 7-2 and Table 7-3 and the steps of the solution procedure in Section 7.5 to show how it can be applied. The results are exactly equal to those were found using the VBA. The optimal results that return the highest system profit are in bold. 
Table 7-4. Example of the manual search for the optimal solution for Scenario 1 (Case 1)

\begin{tabular}{|c|c|c|c|c|c|c|c|c|c|}
\hline$n_{1}$ & $n_{2}$ & $m_{1}$ & $m_{2}$ & $q_{c}$ & $T P_{C-C}^{\text {system }}(\$)$ & Value A & & Value B & Optimal \\
\hline 1 & 1 & 1 & 1 & 7752.4 & $112,766.6$ & & & & \\
\hline 1 & 1 & 1 & 2 & 7558.9 & $112,531.9$ & $112,766.6$ & $>$ & $112,531.9$ & $112,766.6$ \\
\hline 1 & 1 & 2 & 2 & 7632.9 & $112,617.3$ & $112,766.6$ & $>$ & $112,617.3$ & $112,766.6$ \\
\hline 1 & 2 & 1 & 1 & 4626.7 & $113,669.4$ & $112,766.6$ & $<$ & $113,669.40$ & $113,669.4$ \\
\hline 1 & 2 & 1 & 2 & 4474.5 & $113,388.6$ & $113,669.4$ & $>$ & $113,388.6$ & $113,669.4$ \\
\hline 1 & 2 & 2 & 2 & 4531.4 & $113,492.8$ & $113,669.4$ & $>$ & $113,492.8$ & $113,669.4$ \\
\hline 1 & 3 & 1 & 1 & 3377.1 & $113,867.6$ & $113,669.4$ & $<$ & $113,867.6$ & $113,867.6$ \\
\hline 1 & 3 & 1 & 2 & 3253.0 & $113,559.2$ & $113,867.6$ & $>$ & $113,559.2$ & $113,867.6$ \\
\hline 1 & 3 & 2 & 2 & 3298.9 & $113,674.9$ & $113,867.6$ & $>$ & $113,674.9$ & $113,867.6$ \\
\hline 1 & 4 & 1 & 1 & 2695.9 & $113,874.1$ & $113,867.6$ & $<$ & $113,874.1$ & $113,874.1$ \\
\hline 1 & 4 & 1 & 2 & 2590.5 & $113,544.6$ & $113,874.1$ & $>$ & $113,544.6$ & $113,874.1$ \\
\hline 1 & 4 & 2 & 2 & 2629.3 & $113,669.4$ & $113,874.1$ & $>$ & $113,669.4$ & $113,874.1$ \\
\hline 1 & 5 & 1 & 1 & 2264.9 & $113,803.2$ & $113,874.1$ & $>$ & $113,803.2$ & $113,874.1$ \\
\hline 2 & 2 & 1 & 1 & 4859.5 & $113,641.7$ & $113,874.1$ & $>$ & $113,641.7$ & $113,874.1$ \\
\hline 2 & 2 & 2 & 2 & 4754.7 & $113,456.5$ & $113,874.1$ & $>$ & $113,456.5$ & $113,874.1$ \\
\hline 2 & 3 & 1 & 1 & 3551.5 & $113,875.9$ & $113,874.1$ & $<$ & $113,875.9$ & $113,875.9$ \\
\hline 2 & 3 & 1 & 2 & 3414.4 & $113,552.6$ & $113,875.9$ & $>$ & $113,552.6$ & $113,875.9$ \\
\hline 2 & 3 & 2 & 2 & 3464.9 & $113,673.6$ & $113,875.9$ & $>$ & $113,673.6$ & $113,875.9$ \\
\hline 2 & 4 & 1 & 1 & 2836.0 & $113,907.1$ & $113,875.9$ & $<$ & $113,907.1$ & $113,907.1$ \\
\hline 2 & 4 & 1 & 2 & 2719.2 & $113,561.8$ & $113,907.1$ & $>$ & $113,561.8$ & $113,907.1$ \\
\hline 2 & 4 & 2 & 2 & 2762.0 & $113,692.1$ & $113,907.1$ & $>$ & $113,692.1$ & $113,907.1$ \\
\hline 2 & 5 & 1 & 1 & 2382.3 & $113,855.5$ & $113,907.1$ & $>$ & $113,855.5$ & $113,907.1$ \\
\hline 2 & 5 & 2 & 2 & 2317.4 & $113,630.1$ & $113,907.1$ & $>$ & $113,630.1$ & $113,907.1$ \\
\hline 3 & 3 & 1 & 1 & 3668.4 & $113,761.0$ & $113,907.1$ & $>$ & $113,761.0$ & $113,907.1$ \\
\hline 3 & 3 & 2 & 2 & 3577.3 & $113,552.3$ & $113,907.1$ & $>$ & $113,552.3$ & $113,907.1$ \\
\hline 3 & 4 & 1 & 1 & 2927.3 & $113,807.9$ & $113,907.1$ & $>$ & $113,807.9$ & $113,907.1$ \\
\hline 3 & 4 & 2 & 2 & 2849.4 & $113,586.2$ & $113,907.1$ & $>$ & $113,586.2$ & $113,907.1$ \\
\hline 4 & 4 & 1 & 1 & 3004.6 & $113,677.6$ & $113,907.07$ & $>$ & $113,677.6$ & $113,907.1$ \\
\hline
\end{tabular}

The optimal results for the nine cases are summarized in Table 7-5 (next page). The highest system's profit among the four scenarios for each case is shown in bold. 
Table 7-5. Optimal results for the nine cases of the numerical example

\begin{tabular}{|c|c|c|c|c|c|c|c|c|c|c|c|c|c|}
\hline Case & Scenario $i$ & $n_{1}$ & $n_{2}$ & $m_{1}$ & $m_{2}$ & $q_{c}$ & $q_{n c}$ & $q_{s}$ & $q_{v r}$ & $T P_{i}^{S}(\$)$ & $T P_{i}^{v}(\$)$ & $T P_{i}^{b}(\$)$ & $T P_{i}^{\text {system }}(\$)$ \\
\hline \multirow[t]{4}{*}{1} & $\mathrm{C}-\mathrm{C}$ & 2 & 4 & 1 & 1 & 2,836 & & 5,672 & & $15,650.4$ & $38,342.1$ & $59,914.6$ & $113,907.1$ \\
\hline & $\mathrm{NC}-\mathrm{C}$ & 1 & 4 & 1 & 1 & 2,504 & & & 10,017 & $15,608.6$ & $38,899.3$ & $59,766.2$ & $114,274.2$ \\
\hline & $\mathrm{C}-\mathrm{NC}$ & 2 & 4 & 4 & 4 & & 2,592 & 5,184 & & $15,709.9$ & $38,978.3$ & $58,465.9$ & $113,154.1$ \\
\hline & $\mathrm{NC}-\mathrm{NC}$ & 2 & 1 & 2 & 1 & & 2,568 & & 5,137 & $15,648.4$ & $38,970.5$ & $58,466.5$ & $113,085.3$ \\
\hline \multirow[t]{4}{*}{2} & $\mathrm{C}-\mathrm{C}$ & 1 & 4 & 1 & 1 & 2,732 & & 10,926 & & $15,547.8$ & $38,559.8$ & $59,868.6$ & $113,976.2$ \\
\hline & $\mathrm{NC}-\mathrm{C}$ & 1 & 4 & 1 & 1 & 2,558 & & & 10 & $15,820.0$ & $38,858.1$ & $59,793.3$ & $114,471.4$ \\
\hline & $\mathrm{C}-\mathrm{NC}$ & 1 & 4 & 4 & 4 & & 2,503 & 10 & & $15,601.5$ & $39,181.0$ & 57.0 & $113,249.6$ \\
\hline & $\mathrm{NC}-\mathrm{NC}$ & 2 & 4 & 2 & 4 & & 2,624 & & 17 & $15,863.1$ & $38,937.8$ & 5.0 & 65.8 \\
\hline \multirow[t]{4}{*}{3} & $\mathrm{C}-\mathrm{C}$ & 2 & 4 & 1 & 1 & 2,767 & & 3 & & 15 , & 5.3 & 4.3 & 2.1 \\
\hline & $\mathrm{NC}-\mathrm{C}$ & 1 & 5 & 1 & 1 & 2,137 & & & 3 & $15,892.6$ & 1.4 & 1.3 & 5.3 \\
\hline & $\mathrm{C}-\mathrm{NC}$ & 1 & 4 & 4 & 4 & & 2,427 & 9,707 & & 15,5 & 8.5 & 5.9 & 35.5 \\
\hline & $\mathrm{NC}-\mathrm{NC}$ & 2 & 4 & 2 & 4 & & 2,581 & & 5,162 & 15, & 5.0 & 6.2 & 6.4 \\
\hline \multirow[t]{4}{*}{4} & $\mathrm{C}-\mathrm{C}$ & 2 & 4 & 1 & 1 & 2,874 & & 5,747 & & 15 , & 2.8 & 0.6 & 9.1 \\
\hline & $\mathrm{NC}-\mathrm{C}$ & 1 & 4 & 1 & 1 & 2,531 & & & 0,126 & 15, & 9.0 & 0.0 & 4.8 \\
\hline & $\mathrm{C}-\mathrm{NC}$ & 2 & 5 & 5 & 5 & & 2,396 & 5,991 & & $15,926.9$ & 34.3 & 4.9 & 6.1 \\
\hline & $\mathrm{NC}-\mathrm{NC}$ & 2 & 5 & 2 & 5 & & 2,365 & & 911 & $15,842.4$ & 78.2 & 3.5 & 84.1 \\
\hline \multirow[t]{4}{*}{5} & $\mathrm{C}-\mathrm{C}$ & 1 & 3 & 1 & 1 & 3,475 & & 10,424 & & 15 , & 4.2 & 4.3 & 36.7 \\
\hline & $\mathrm{NC}-\mathrm{C}$ & 1 & 4 & 1 & 1 & 2,587 & & & 10,347 & $15,839.3$ & 27.9 & 7.5 & 74.7 \\
\hline & $\mathrm{C}-\mathrm{NC}$ & 1 & 2 & 5 & 5 & & 2,310 & 11,548 & & $15,818.8$ & $39,650.7$ & $58,460.1$ & 29.7 \\
\hline & $\mathrm{NC}-\mathrm{NC}$ & 2 & 5 & 2 & 5 & & 2,429 & & 6,071 & $16,085.5$ & $39,440.9$ & $58,466.0$ & 92.4 \\
\hline \multirow[t]{4}{*}{6} & $\mathrm{C}-\mathrm{C}$ & 2 & 3 & 1 & 1 & 3,525 & & 5,288 & & $15,611.8$ & $38,217.9$ & 88.0 & 17.7 \\
\hline & $\mathrm{NC}-\mathrm{C}$ & 1 & 4 & 1 & 1 & 2,565 & & & 10,260 & $15,824.8$ & $38,875.5$ & 6.8 & 7.1 \\
\hline & $\mathrm{C}-\mathrm{NC}$ & 2 & 5 & 5 & 5 & & 2,335 & 5,837 & & $15,948.1$ & $39,260.5$ & $58,461.8$ & 70.5 \\
\hline & $\mathrm{NC}-\mathrm{NC}$ & 2 & 5 & 2 & 5 & & 2,379 & & 5,947 & $16,056.5$ & $39,310.7$ & $58,464.2$ & $113,831.4$ \\
\hline \multirow[t]{4}{*}{7} & $\mathrm{C}-\mathrm{C}$ & 1 & 3 & 1 & 1 & 3,126 & & 9,379 & & $15,245.7$ & $38,838.7$ & 56.6 & 41.1 \\
\hline & $\mathrm{NC}-\mathrm{C}$ & 1 & 4 & 1 & 1 & 2,123 & & & 8,490 & $15,309.8$ & $39,231.8$ & $58,920.8$ & $113,462.4$ \\
\hline & $\mathrm{C}-\mathrm{NC}$ & 2 & 5 & 5 & 5 & & 2,326 & 5,815 & & $15,888.3$ & $39,523.1$ & $58,228.7$ & $113,640.0$ \\
\hline & $\mathrm{NC}-\mathrm{NC}$ & 2 & 5 & 2 & 5 & & 2,297 & & 5,742 & $15,805.8$ & $39,515.7$ & $58,229.5$ & $113,551.0$ \\
\hline \multirow[t]{4}{*}{8} & $\mathrm{C}-\mathrm{C}$ & 1 & 3 & 1 & 1 & 3,160 & & 9,479 & & $15,350.6$ & $38,819.5$ & $59,159.6$ & $113,329.7$ \\
\hline & $\mathrm{NC}-\mathrm{C}$ & 1 & 4 & 1 & 1 & 2,155 & & & 620 & $15,489.9$ & $39,214.2$ & $58,932.8$ & $113,636.9$ \\
\hline & $\mathrm{C}-\mathrm{NC}$ & 1 & 5 & 5 & 5 & & 2,244 & 11,218 & & $15,780.8$ & $39,691.2$ & $58,230.1$ & $113,702.1$ \\
\hline & $\mathrm{NC}-\mathrm{NC}$ & 2 & 5 & 2 & 5 & & 2,355 & & $J, 000$ & $16,042.3$ & $39,483.4$ & $58,227.5$ & $113,753.2$ \\
\hline \multirow[t]{4}{*}{9} & & 1 & 3 & 1 & 1 & 3,068 & & 9,203 & & 15,3 & $38,625.5$ & 0.9 & 79.4 \\
\hline & & 1 & 4 & 1 & 1 & 2,142 & & & 8,569 & $15,477.3$ & $39,163.8$ & $58,928.2$ & $113,569.3$ \\
\hline & $\mathrm{C}-\mathrm{NC}$ & 2 & 5 & 5 & 5 & & 2,270 & 5,674 & & $15,908.4$ & $39,302.0$ & $58,229.9$ & $113,440.3$ \\
\hline & $\mathrm{NC}-\mathrm{NC}$ & 2 & 5 & 2 & 5 & & 2,310 & & 5,774 & $16,013.9$ & $39,354.0$ & $58,229.2$ & $113,597.0$ \\
\hline
\end{tabular}

To find the optimal solution seen in Table 7-5, the values of the parameters in Table 7-2 and Table 7-3 plugged into the equations developed in Sections 7.1, 7.2 and 7.3, summarized in Table 7-6 (next page), and then the profit functions are optimized using a nested loop that searches for the values of the decision variables $\left(n_{1}, n_{2}, m_{1}\right.$, and $\left.m_{2}\right)$ using the solution procedure developed in Section 7.5 and in a similar manner to that of Jaber and Goyal (2008). Two conditions to be met while performing the search; (1) $n_{2} \geq n_{1}$ to avoid shortages, and (2) $m_{2} \geq m_{1}$ to make sure that the vendor does not run out of cash. For Scenarios 2, 3, and 4, the conditions are: (1) 
$n_{2} \geq n_{1}$ and (2) $m_{2} \geq n_{1}$, (1) $n_{2} \geq n_{1}$ and (2) $n_{2} \geq m_{1}$, and (1) $n_{2} \geq n_{1}$, respectively, where $n_{1}, n_{2}, m_{1}$, and $m_{2}$ are integers.

Table 7-6. Number of equations used in the calculation of the optimal quantity and the total profit for each scenario

\begin{tabular}{lccccccc}
\hline Scenario $i$ & $q_{c, i}^{*}$ & $q_{n c, i}^{*}$ & $q_{s, i}^{*}$ & $q_{v r, i}^{*}$ & $T P_{i}^{S}$ & $T P_{i}^{v}$ & $T P_{i}^{b}$ \\
\hline C-C & $7-4$ & & $7-5$ & & $7-1$ & $7-2$ & $7-3$ \\
NC-C & $7-9$ & & & $7-5^{\mathrm{a}}$ & $7-6$ & $7-7$ & $7-8$ \\
C-NC & & $7-13$ & $7-5^{\mathrm{b}}$ & & $7-10$ & $7-11$ & $7-12$ \\
NC-NC & & $7-17$ & & $7-5^{\mathrm{c}}$ & $7-14$ & $7-15$ & $7-16$ \\
\hline
\end{tabular}

a replace $q_{s, i}^{*}$ with $q_{v r, i}^{*}{ }^{\text {b }}$ replace $q_{c, i}^{*}$ with $q_{n c, i}^{*}{ }^{\text {c }}$ replace $q_{s, i}^{*}$ with $q_{v r, i}^{*}$ and $q_{c, i}^{*}$ with $q_{n c, i}^{*}$

The results in Table 7-5 show that the NC-C Scenario records the highest total system profit for Cases 1 to 6 with the C-C Scenario being the second best for Cases 1 to 5. For Case 7, the C-NC Scenario achieves the highest system's profit where the NC-NC Scenario records the best results for Cases 8 and 9. The results imply that the scenario with the highest profit may not be the best for some players. As this chapter assumes a centralized decision-making strategy, the final decision is made based on the system's profit.

For a comprehensive view of the effect of the variable costs on the total system's profit, Table 7-7 (next page) provides a breakdown of these costs for the nine cases. The results show that the system's setup cost is different from one case to another as it depends on the number and size of shipments. Most cases show that adopting the CS policy reduces the setup cost. The storageholding cost of raw material at the vendor's side is the highest when a CS agreement is adopted by the supplier, whereas the traditional policy is shown to reduce this cost. This is because, in a CS policy, the supplier ships larger quantities to the vendor than in the traditional policy. Setting a storage capacity reduces this cost. The total storage-holding cost of the system's finished items is shown to increase for the traditional policy and decrease for the CS or for a combination of both policies. However, it increases the system's financial-holding cost of finished items at the warehouse of the downstream player. The reasons behind increasing the financial-holding cost are: (1) the financial-holding cost depends on the value of the product, which increases as it moves downstream in the chain, (2) if there is a CS agreement, the downstream player pays the upstream one after withdrawing the items, which affects the upstream player, especially if the number of payments is less frequent or at the end of the cycle. However, the CS showed to decrease the system's financial-holding cost as the upstream player makes frequent shipments of items, thus 
reducing inventory at its side, to the downstream player where it is cheaper to store them. Table 7-7 also shows that when the traditional policy is adopted, the financial-holding cost of the raw material reaches a maximum and a minimum when a CS policy is adopted between the vendor and the buyer as the CS policy results in consuming the raw material faster. The results also show that the CS policy has lower ordering costs than the traditional policy as larger and less frequent shipments are made when compared to the traditional policy. Finally, the results show that the transaction cost is higher for a traditional policy as there are more transactions performed than in a CS policy.

Table 7-7. Breakdown of all variable costs that affect the total system profit for all cases

\begin{tabular}{|c|c|c|c|c|c|c|c|c|c|}
\hline Case & Scenario & $\begin{array}{l}\text { Setup } \\
(\$)\end{array}$ & $\begin{array}{l}\text { Storage- } \\
\text { holding of } \\
\text { RM }(\$)\end{array}$ & $\begin{array}{l}\text { Storage- } \\
\text { holding of } \\
\text { FP }(\$)\end{array}$ & $\begin{array}{l}\text { Financial- } \\
\text { holding of } \\
\text { RM }(\$)\end{array}$ & $\begin{array}{l}\text { Financial- } \\
\text { holding of FP } \\
\text { at player's } \\
\text { warehouse (\$) }\end{array}$ & $\begin{array}{c}\text { Financial- } \\
\text { holding of FP } \\
\text { at downstream } \\
\text { player's } \\
\text { warehouse }(\$)\end{array}$ & $\begin{array}{l}\text { Ordering } \\
(\$)\end{array}$ & $\begin{array}{c}\text { Transaction } \\
(\$)\end{array}$ \\
\hline \multirow[t]{4}{*}{1} & $\mathrm{C}-\mathrm{C}$ & $2,644.6$ & 250.5 & $2,537.4$ & - & 59.8 & $3,436.6$ & $1,234.1$ & 11.0 \\
\hline & $\mathrm{NC}-\mathrm{C}$ & $2,994.8$ & 67.1 & $2,494.9$ & 56.3 & 86.7 & $2,623.5$ & $1,197.9$ & 5.0 \\
\hline & C-NC & $2,893.4$ & 229.0 & $3,078.4$ & - & 704.9 & 250.7 & $1,350.2$ & 12.1 \\
\hline & $\mathrm{NC}-\mathrm{NC}$ & $2,920.1$ & 137.6 & $3,311.0$ & 115.6 & 733.2 & - & $1,362.7$ & 14.6 \\
\hline \multirow[t]{4}{*}{2} & $\mathrm{C}-\mathrm{C}$ & $2,745.6$ & 292.7 & $2,444.1$ & - & 71.3 & $3,353.3$ & $1,098.3$ & 11.4 \\
\hline & $\mathrm{NC}-\mathrm{C}$ & $2,932.1$ & 68.5 & $2,370.4$ & 57.6 & 88.5 & $2,679.7$ & $1,172.8$ & $4 . .9$ \\
\hline & $\mathrm{C}-\mathrm{NC}$ & $2,996.6$ & 268.2 & $2,972.4$ & - & 693.1 & 281.6 & $1,198.6$ & 12.5 \\
\hline & $\mathrm{NC}-\mathrm{NC}$ & $2,858.7$ & 140.5 & $3,199.6$ & 118.1 & 748.9 & - & $1,334.1$ & 14.3 \\
\hline \multirow[t]{4}{*}{3} & C-C & $2,710.9$ & 488.8 & $2,423.3$ & - & 58.3 & $3,352.6$ & $1,265.1$ & 11.3 \\
\hline & $\mathrm{NC}-\mathrm{C}$ & $2,808.2$ & 114.5 & $2,360.5$ & 48.1 & 83.9 & $2,769.1$ & $1,357.3$ & 4.7 \\
\hline & $\mathrm{C}-\mathrm{NC}$ & $3,090.4$ & 520.0 & $2,882.1$ & - & 672.1 & 273.0 & $1,236.2$ & 12.9 \\
\hline & $\mathrm{NC}-\mathrm{NC}$ & $2,906.1$ & 276.5 & $3,147.4$ & 116.1 & 136.7 & - & $1,356.2$ & 14.5 \\
\hline \multirow[t]{4}{*}{4} & $\mathrm{C}-\mathrm{C}$ & $2,609.9$ & 253.9 & $2,468.6$ & - & 60.6 & $3,482.4$ & $1,217.9$ & 10.9 \\
\hline & $\mathrm{NC}-\mathrm{C}$ & $2,962.8$ & 67.8 & $2,431.5$ & 57.0 & 87.6 & $2,651.9$ & $1,185.1$ & 4.9 \\
\hline & C-NC & $2,503.9$ & 264.6 & $2,637.2$ & - & 723.9 & 276.2 & $1,377.2$ & 12.5 \\
\hline & $\mathrm{NC}-\mathrm{NC}$ & $2,537.5$ & 158.3 & $2,902.3$ & 133.0 & 754.3 & - & $1,395.6$ & 14.8 \\
\hline \multirow[t]{4}{*}{5} & $\mathrm{C}-\mathrm{C}$ & $2,878.1$ & 279.2 & $2,393.6$ & - & 82.0 & $3,245.9$ & 911.4 & 9.6 \\
\hline & $\mathrm{NC}-\mathrm{C}$ & $2,899.3$ & 69.3 & $2,304.8$ & 58.2 & 89.6 & $2,710.0$ & $1,159.7$ & 4.8 \\
\hline & $\mathrm{C}-\mathrm{NC}$ & $2,597.9$ & 309.3 & $2,541.8$ & - & 712.2 & 311.8 & $1,255.6$ & 13.0 \\
\hline & $\mathrm{NC}-\mathrm{NC}$ & $2,470.6$ & 162.6 & $2,769.8$ & 136.6 & 774.8 & - & $1,358.8$ & 14.4 \\
\hline \multirow[t]{4}{*}{6} & $\mathrm{C}-\mathrm{C}$ & $2,836.7$ & 467.2 & $2,378.9$ & - & 69.9 & $3,251.5$ & $1,087.4$ & 9.5 \\
\hline & $\mathrm{NC}-\mathrm{C}$ & $2,923.9$ & 137.4 & $2,285.4$ & 57.7 & 88.8 & $2,687.2$ & $1,169.6$ & 4.9 \\
\hline & C-NC & $2,569.9$ & 515.7 & $2,514.7$ & - & 705.3 & 269.1 & $1,413.4$ & 12.8 \\
\hline & $\mathrm{NC}-\mathrm{NC}$ & $2,522.3$ & 318.6 & $2,713.0$ & 133.8 & 758.9 & - & $1,387.3$ & 14.7 \\
\hline \multirow[t]{4}{*}{7} & $\mathrm{C}-\mathrm{C}$ & $3,198.6$ & 251.2 & $2,956.5$ & - & 73.8 & $2,920.7$ & $1,012.9$ & 10.7 \\
\hline & $\mathrm{NC}-\mathrm{C}$ & $3,533.5$ & 56.9 & $2,660.4$ & 47.8 & 73.5 & $2,223.6$ & $1,413.4$ & 5.9 \\
\hline & $\mathrm{C}-\mathrm{NC}$ & $2,579.7$ & 256.9 & $2,792.4$ & - & 702.7 & 268.1 & $1,418.8$ & 12.9 \\
\hline & $\mathrm{NC}-\mathrm{NC}$ & $2,612.4$ & 153.8 & $3,048.8$ & 129.2 & 732.7 & - & $1,436.8$ & 15.2 \\
\hline \multirow[t]{4}{*}{8} & $\mathrm{C}-\mathrm{C}$ & $3,165.0$ & 253.9 & $2,898.9$ & - & 74.5 & $2,951.7$ & $1,002.2$ & 10.5 \\
\hline & $\mathrm{NC}-\mathrm{C}$ & $3,480.4$ & 57.7 & $2,551.1$ & 48.5 & 74.6 & $2,257.5$ & $1,392.2$ & 5.8 \\
\hline & $\mathrm{C}-\mathrm{NC}$ & $2,674.2$ & 300.5 & $2,693.7$ & - & 691.9 & 302.9 & $1,292.5$ & 13.4 \\
\hline & $\mathrm{NC}-\mathrm{NC}$ & $2,547.4$ & 157.7 & $2,921.8$ & 132.5 & 751.4 & - & $1,401.1$ & 14.9 \\
\hline \multirow[t]{4}{*}{9} & $\mathrm{C}-\mathrm{C}$ & $3,259.9$ & 493.0 & $2,814.5$ & - & 72.4 & $2,865.8$ & $1,032.3$ & 10.9 \\
\hline & $\mathrm{NC}-\mathrm{C}$ & $3,500.9$ & 114.8 & $2,536.1$ & 48.2 & 74.2 & $2,244.3$ & $1,400.4$ & 5.8 \\
\hline & C-NC & $2,643.7$ & 501.3 & $2,671.4$ & - & 685.6 & 261.6 & $1,454.1$ & 13.2 \\
\hline & $\mathrm{NC}-\mathrm{NC}$ & $2,597.6$ & 309.3 & $2,865.3$ & 129.9 & 736.9 & - & $1,428.7$ & 15.2 \\
\hline
\end{tabular}

(RM: raw material, and FP: finished product) 


\subsection{Sensitivity Analysis}

The nine cases provided in the numerical example are investigated to identify the parameters that affect the performance of the players and the system the most; note that when changing the values of one parameter, the other parameters are kept fixed at their initial values. The following ratios were considered in the analysis: $0.1 \leq D / P_{v} \leq 0.9,0.5 \leq S_{v} / S_{s} \leq 2,0.5 \leq O_{b} / O_{v} \leq 2$, and $0 \leq$ $I_{b} / I_{v} \leq 2$. The results of the analysis are summarized as follow:

Demand over production $D / P_{v}$ rates: the results showed that the total profit of the system increases as the ratio of $D / P_{v}$ increases in all cases. It also showed that the NC-C Scenario achieved the highest profit in $80 \%$ of the tests performed where the $\mathrm{C}-\mathrm{C}$, the C-NC and the NCNC Scenarios had the highest in $6.7 \%, 8.9 \%$ and $4.4 \%$, respectively.

Vendor's setup over supplier's setup $\boldsymbol{S}_{\boldsymbol{v}} / \boldsymbol{S}_{\boldsymbol{s}}$ costs: the results showed that as the ratio of $S_{v} / S_{S}$ increases, the total profit decreases for all scenarios in all cases. This shows that it is always better to have the setup cost of the vendor less than the setup cost of its supplier. This might be done by sending semi-finished items to the vendor instead of raw material or by eliminating the nonproductive activities that increase the vendor's setup cost. The results also showed that the NC-C Scenario achieved the highest profit in $66.7 \%$ of the instances followed by the NC-NC and C-NC Scenarios in $22.2 \%$ and $11.1 \%$, respectively.

Buyer's ordering over vendor's ordering $\boldsymbol{O}_{b} / \boldsymbol{O}_{v}$ costs: the results showed that it is better to have a buyer ordering cost that is less than that of the vendor as the system's profit decreases when the ratio of $O_{b} / O_{v}$ increases for all scenarios in all cases. For Cases 1 to 6 , the NC-C Scenario achieved the highest profit as $O_{b} / O_{v}$ varied from 0.5 to 2 where the highest for Case 7 was achieved by the C-NC Scenario. For Cases 8 and 9, the NC-NC Scenario achieved the highest profit when $O_{b} / O_{v} \leq$ 1.5 where the NC-C Scenario achieved the highest when $O_{b} / O_{v}>1.5$.

Buyer's interest over vendor's interest $I_{b} / I_{v}$ rates: the results showed that for the C-C and NC$C$ Scenarios, the system's profit increases as $I_{b} / I_{v}$ increases in all cases where it decreases for the other scenarios. The NC-C Scenario had the highest system's profit in $64.5 \%$ of the instances while Scenarios NC-NC and C-NC had the highest profits in $22.2 \%$ and $13.3 \%$ of the instances, respectively. 
In summary, the CS policy shows to serve the system better than the traditional policy in many cases. It shows to increase the system's profitability when the buyer's demand is high and ensures products' availability. It gives the downstream players the opportunity to invest the revenues they generated from sales for a period of time, from the time a shipment is received until it is paid for. Hence, it helps in generating more profit for the player and the system especially when the interest rate for investment for the downstream player is high. Moreover, it was found that the CS policy performs better than the traditional policy only if the storage-holding cost of the downstream player (especially the buyer) is less than or equal to the upstream one. Otherwise, the traditional policy is preferred. Although a combined coordination policy (NC-C) was shown to reflect a higher system profit, the system's profit is insignificantly higher than when a C-C policy is adopted. This suggests that adopting a CS agreement by all players in the supply chain might be better than the traditional policy or a combined one, especially when the fluctuation of the demand is high and cannot be predicted by the supplier and the vendor. This improves collaboration, sharing information and transparency between the players in the supply chain, which has a good impact on the system. It helps in providing better services, increasing product availability, reducing forecasting errors, and minimizing the disposal of unused, unsold or perishable items. The CS also showed to decrease the number of orders made by the downstream player, which results in a reduction in its related cost.

\subsection{Managerial Insights}

This section provides general managerial insights for managers and/or decision makers to help them in taking the right decision.

1. When the system consists of more than two players, adopting the consignment stock contract by all players might not return the highest system profit. Therefore, choosing the best policy between two adjacent players could be different from the other two and depends on the exact value of the system's parameters.

2. Consignment stock helps in better collaboration between the players which increases sales monitoring, product availability, and customers' satisfaction.

3. Consignment stock is recommended when the storage-holding cost of the downstream player is less than or equal to the upstream player. 
4. Consignment stock helps the upstream player in managing its production, reducing the forecasting errors and minimizing the disposal of unused, unsold or expired products.

5. Although a consignment stock contract increases some costs for the downstream player such as storage-holding and insurance, it helps in reducing the costs of ordering, financialholding, disposal of unused, unsold, or expired items. It also helps given the downstream player the opportunity to invest its sales revenue until a payment is due. This may be an incentive for a buyer (downstream) to enter into a CS agreement with its vendor (upstream).

\subsection{Conclusion}

This chapter developed a three-level (supplier-vendor-buyer) supply chain system and investigated it for four coordination scenarios. The scenarios are combinations of consignment stock and traditional coordination policies. The chapter assumed that a downstream player makes equal-sized payments to the upstream player at equal time intervals when adjacent players have a consignment stock agreement. In a traditional coordination agreement, a payment is received upon the delivery of a shipment. Each coordination scenario has nine cases representing the storage-holding costs. A sensitivity analysis was performed to investigate the effects of varying different cost factors on the developed models (scenarios). Some managerial insights were provided to help decision makers in choosing the best policy or scenario that serves and maximizes the profit of the supply chain system.

The numerical results showed that the best scenario for the system might be different from the one preferred by a player as it depends on the storage-holding cost. Coordination may shift the savings/profits to one or more players, others will lose. A losing party can receive a share of the supply chain profit and/or a price discount on purchases. The results did not recommend consignment agreement between the vendor and the buyer when the storage-holding cost of the latter is higher.

A sensitivity analysis was performed to determine the parameters that have the most effect on the system's profitability. Although adopting a traditional coordination policy among the players in some instances produced the highest results, a combined policy, followed by a consignment agreement among all players was shown to return the highest system's profit in most cases. The results showed that changes in the demand rate, the vendor's setup and the buyer's ordering costs, 
or the buyer's interest rate affect the system's profit and the order policies. This suggests that it is important to have complete information regarding the values of the input parameters for the three players.

In general, adopting a consignment agreement ensures better management and services. It enhances collaboration between adjacent players as it needs frequent sharing of information about the product flow, which results in sales monitoring and production management. Moreover, consignment stock ensures product availability, especially when the demand fluctuates, which results in greater customer satisfaction. In addition, since payment is delayed when adopting a consignment agreement, the downstream player has the opportunity to invest its sales' revenue before making a payment. Similarly, the upstream player benefits from adopting the consignment agreement if it (1) does not have enough space to store products, (2) wants to reduce its storageholding costs, (3) sells a slow-moving product (it is newly launched or its demand is low), (4) enters a highly competitive market and wants to sell a product to a player that already has a supplier for the same product, (5) plans to reduce its transportation costs or it does not have a sufficient fleet to handle it, or to reduce greenhouse gases emissions and their related costs, and finally (6) wants to sell the product to a start-up business to support it and retain it as a customer.

This chapter can be extended by considering a price dependent demand, a non-zero lead time for shipments and/or payments, unequal-sized payments at equal or unequal size intervals when buyer's demand is stochastic or when considering multiple products. A net present value approach could also be used to model the problem of this chapter. This is left as a future research exercise.

The next chapter investigates the individual or combined effects of adopting a consignment stock agreement or a traditional coordination agreement in a three-level supply chain system with multiple suppliers and buyers. 


\section{Chapter 8: Investigation of a consignment stock and a traditional inventory policy in a three-level supply chain system with multiple suppliers and multiple buyers}

Inventory represents a significant portion of the costs of a supply chain, and managing it well is primary for the success of a supply chain management program. Inventory management (IM) includes but is not limited to, the proper management of ordering, storage and use of materials that are used in production, and the quantities of finished products. For these reasons, researchers have intensively investigated and published different studies describing different inventory situations, and developing policies and models or suggesting methods to lower inventory costs, one of which is consignment stock (CS). A CS policy has been shown to be effective in reducing supply chain (SC) costs and in eliminating the temporary out-of-stock situations by allowing an upstream (vendor) player to store its products at a downstream (buyer's) facility.

Trade credit significantly affects the management of inventory and a firm's profits (Wilson and Summers, 2002; Tirole, 2006). Delay-in-Payments, a form of trade credit, has been studied by many researchers for different inventory and supply chain situations. It entices a buyer to order in larger quantities from its vendor. As more items move to the side of the retailer, a vendor saves on storage and frees space. The vendor incurs a capital cost because of the money tied up in inventory for a period extending from the time the buyer receives a shipment to the time it settles its owed balance to the vendor. The buyer benefits from a reduction in its holding cost and by having the opportunity to invest its sales revenue over the delay period (usually interest-free). The literature shows that the length of the delay period is fixed and always set by the vendor. Jaber and Osman (2006) were the first to treat it and extended the delay period (interest charged) as decision variables among others.

To the best of author's knowledge and from the review of the CS literature, no study yet has modelled a CS three-level supply chain that consists of multiple suppliers (produce parts), a vendor (stores parts and produces a final product) that sells a final product to multiple buyers, especially, when delay-in-payments and equal-sized payments made at equal time intervals are considered. Therefore, this chapter develops and investigates four coordination scenarios for a three-level supply chain system consisting of multiple suppliers, a vendor, and multiple buyers. One scenario considers consignment stock (CS) agreements between the suppliers and the vendor and between 
the vendor and the buyer. Another scenario considers traditional coordination policies (TP) between adjacent players in the supply chain. The two remaining scenarios are combinations of the two; i.e., CS agreements (TP) between the suppliers and the vendor and TP (CS agreements) between the vendor and the buyer. The scenarios also consider trade credit where an upstream player offers a delay-in-payment to a downstream player. Equal-sized payments scheme made at equal intervals is adopted.

The four developed scenarios are:

1. No Consignment - No Consignment (NC - NC) Scenario: all players adopt TP.

2. No Consignment - Consignment $(\mathrm{NC}-\mathrm{C})$ Scenario: the TP is adopted by all suppliers and the vendor, and CS agreement by the vendor and all buyers.

3. Consignment - No Consignment $(\mathrm{C}-\mathrm{NC})$ Scenario: the CS agreement is adopted by all suppliers and the vendor, and TP by the vendor and all buyers.

4. Consignment - Consignment $(\mathrm{C}-\mathrm{C})$ Scenario: all players adopt $\mathrm{CS}$ agreements.

This chapter assumes that a single vendor signs coordination contracts with several suppliers. Each supplier provides the vendor with a different part that is needed to produce the final product to be sold to multiple buyers.

To illustrate, Figure 8-1 (next page) represents a general diagram of a three stages supply chain system. Stage 1 consists of multiple non-identical suppliers that produce different parts and ship them to the vendor's warehouse. Stage 2 consists of a vendor that uses the parts received from the suppliers to produce and store items of a product. The vendor then ships the items to the buyers in batches at specific times in accordance with the signed coordination agreement (TP or CS) between them. Stage 3 consists of multiple buyers that store the items of the finished product in their warehouse and sell them to consumers. 


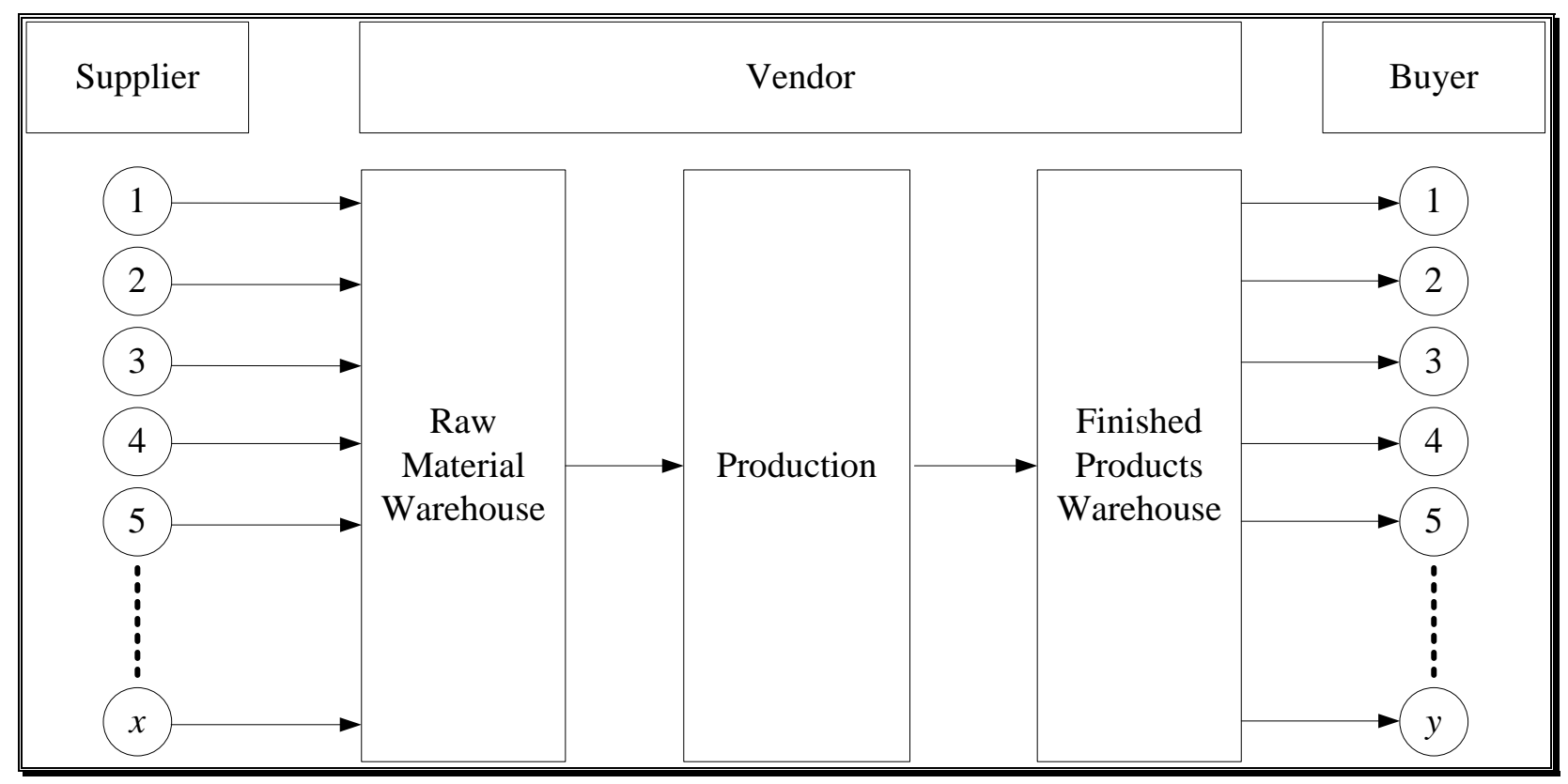

Figure 8-1. General diagram for a three-level supply chain system with multiple-suppliers and multiple-buyers

This chapter assumes that a single vendor signs coordination contracts with several suppliers. Each supplier furnishes the vendor with a different part that is needed to produce a final product to be sold to multiple buyers. The following assumptions are made in this chapter, which are in line with the literature:

1) The demand rates for all buyers are different, deterministic and constant.

2) The production rates of all suppliers are faster than the rate(s) at which the vendor uses/consumes the parts supplied by them to produce the final product. The vendor's production rate is faster than the demand rates for the buyers. These assumptions guarantee that no shortages occur in the supply chain.

3) The suppliers (buyers) adopt the same type of the contract (CS or TP) with the vendor. The type of contract between the vendor and the buyers could be different than the one between the vendor and the suppliers.

The following notations were used for the suppliers:

$x \quad$ number of suppliers, where $j=1,2,3, \ldots x$

$S_{S_{j}} \quad$ supplier $j$ set-up cost $(\$ /$ set-up)

$P_{s_{j}} \quad$ supplier $j$ production rate (units/year) 
$\gamma_{j} \quad$ number of components in a part furnished by supplier $j$; where $\gamma_{j} \geq 1$ and integer

$I_{s_{j}} \quad$ supplier $j$ investment interest rate (\%/year)

$I_{s_{j}}^{c} \quad$ supplier $j$ interest charged rate for vendor (\%/year)

$c_{s_{j}}^{p} \quad$ supplier $j$ component purchasing cost (\$/unit)

$c_{s_{j}}^{p r} \quad$ supplier $j$ production cost (\$/unit)

$c_{s_{j}}^{s} \quad$ part selling price per unit furnished by supplier $j$ (\$/unit); where $c_{s_{j}}^{s}=$ $\left(1+a_{s_{j}}\right)\left(c_{s_{j}}^{p}+c_{s_{j}}^{p r}\right)$ and $a_{s_{j}}$ is the marginal profit for supplier $j$

$h_{s_{j}}^{s} \quad$ supplier $j$ part storage-holding cost (\$/unit/year)

$h_{s_{j}}^{f_{1}} \quad$ supplier $j$ part in-house financial-holding cost (\$/unit/year); where $h_{s_{j}}^{f_{1}}=c_{s_{j}}^{p r} I_{s_{j}}$

$h_{s_{j}}^{f_{2}} \quad$ supplier $j$ part out-house financial-holding cost (\$/unit/year); where $h_{s_{j}}^{f_{2}}=c_{s_{j}}^{s} I_{s_{j}}$

The following notations were used for the vendor:

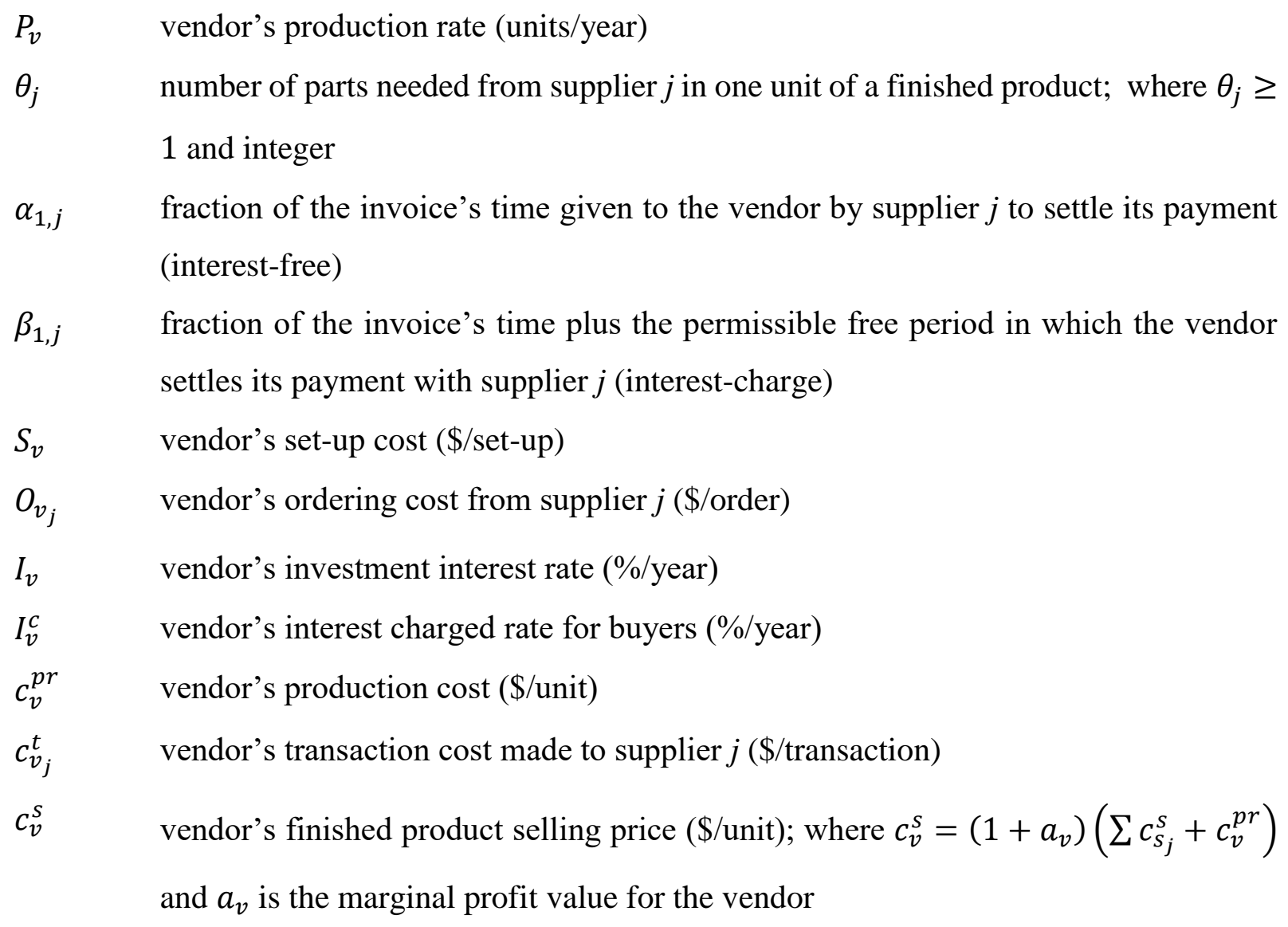


$h_{v_{j}}^{s, r} \quad$ vendor's storage-holding cost for a part received from supplier $j$ (\$/unit/year)

$h_{v_{j}}^{f, r} \quad$ vendor's financial-holding cost for a part received from supplier $j$ (\$/unit/year); where $h_{v_{j}}^{f, r}=c_{s_{j}}^{s} I_{v}$

$h_{v}^{s, f} \quad$ vendor's product storage-holding cost (\$/unit/year)

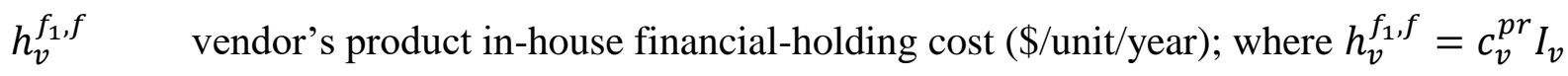

$h_{v}^{f_{2}, f} \quad$ vendor's product out-house financial-holding cost (\$/unit/year); where $h_{v}^{f_{2}, f}=c_{v}^{s} I_{v}$

The following notations were used for the buyers:

$y \quad$ number of buyers, where $i=1,2,3, \ldots y$

$d_{i} \quad$ Buyer's demand rate (units/year)

$\alpha_{2, i} \quad$ fraction of the invoice's time given to buyer $i$ by the vendor to settle its payment (interest-free)

$\beta_{2, i} \quad$ fraction of the invoice's time plus the permissible free period in which buyer $i$ settles its payment with the vendor (interest-charge)

$O_{b_{i}} \quad$ buyer $i$ ordering cost (\$/order)

$I_{b_{i}} \quad$ buyer $i$ investment interest rate (\%/year)

$h_{b_{i}}^{S} \quad$ buyer $i$ product storage-holding cost (\$/unit/year)

$h_{b_{i}}^{f} \quad$ buyer $i$ product financial-holding cost (\$/unit/year); where $h_{b_{i}}^{f}=c_{v}^{s} I_{b_{i}}$

$c_{b_{i}}^{S} \quad$ buyer $i$ product selling price (\$/unit); where $c_{b_{i}}^{S}=\left(1+a_{b_{i}}\right) c_{v}^{S}$ and $a_{b_{i}}$ is the marginal profit value for buyer $i$

$c_{b_{i}}^{t} \quad$ buyer $i$ transaction cost (\$/transaction)

The following notations were used for the decision variables:

$\begin{array}{ll}n_{1, j} & \text { number of shipments from supplier } j \text { to the vendor } \\ n_{2, i} & \text { number of shipments from the vendor to buyer } i \\ m_{1, j} & \text { number of payments made by the vendor to supplier } j \\ m_{2, i} & \text { number of payments made by buyer } i \text { to the vendor } \\ T_{z}^{*} & \text { optimum cycle time for the system for Scenario } z=1,2,3 \text { and } 4\end{array}$ 
Next sections are divided as follow; Section 8.1 represents a brief description of the traditional and the consignment stock policies, Section 8.2 is for Scenario 1, Section 8.3 represents Scenario 2, Section 8.4 describes Scenario 3, Section 8.5 is for Scenario 4, Section 8.6 presents the solution procedure, Section 8.7 illustrates a numerical example, Section 8.8 performs a sensitivity analysis, Section 8.9 provides some managerial insights. Section 8.10 is for the conclusion and future work.

\subsection{Brief description of the traditional policy (TP) and the consignment stock (CS)}

Before proceeding with the mathematical modeling, a brief description of the TP and the CS, which are used in the mathematical formulation, are explained in Sections 8.1.1 and 8.1.2.

\subsubsection{Traditional policy (TP)}

Figure 8-2 depicts the general behavior of the inventory and supply chain system when a vendor and a buyer operating under TP that is considered to develop the mathematical modeling. In the figure, an upstream player $u$ (supplier or vendor) produces $q_{u}$ units and ships it to the downstream player $d$ (vendor or buyer) $q_{d}$ units during production. Player $u$ stops production when its inventory level reaches $n q_{d}$ units. It continues shipping $q_{d}$ units every $q_{d} / D$ units of time. Player $d$ pays Player $u$ when it receives an invoice. The number of payments is equal to the number of shipments. There are three cases of delay-in-payments. Player $d$ pays Player $u$ when it receives a shipment, by the end of a permissible delay (interest-free) period, or past the delay period (interest is charged). In the third case, Player $u$ charges Player $d$ interest on the balance it owes.

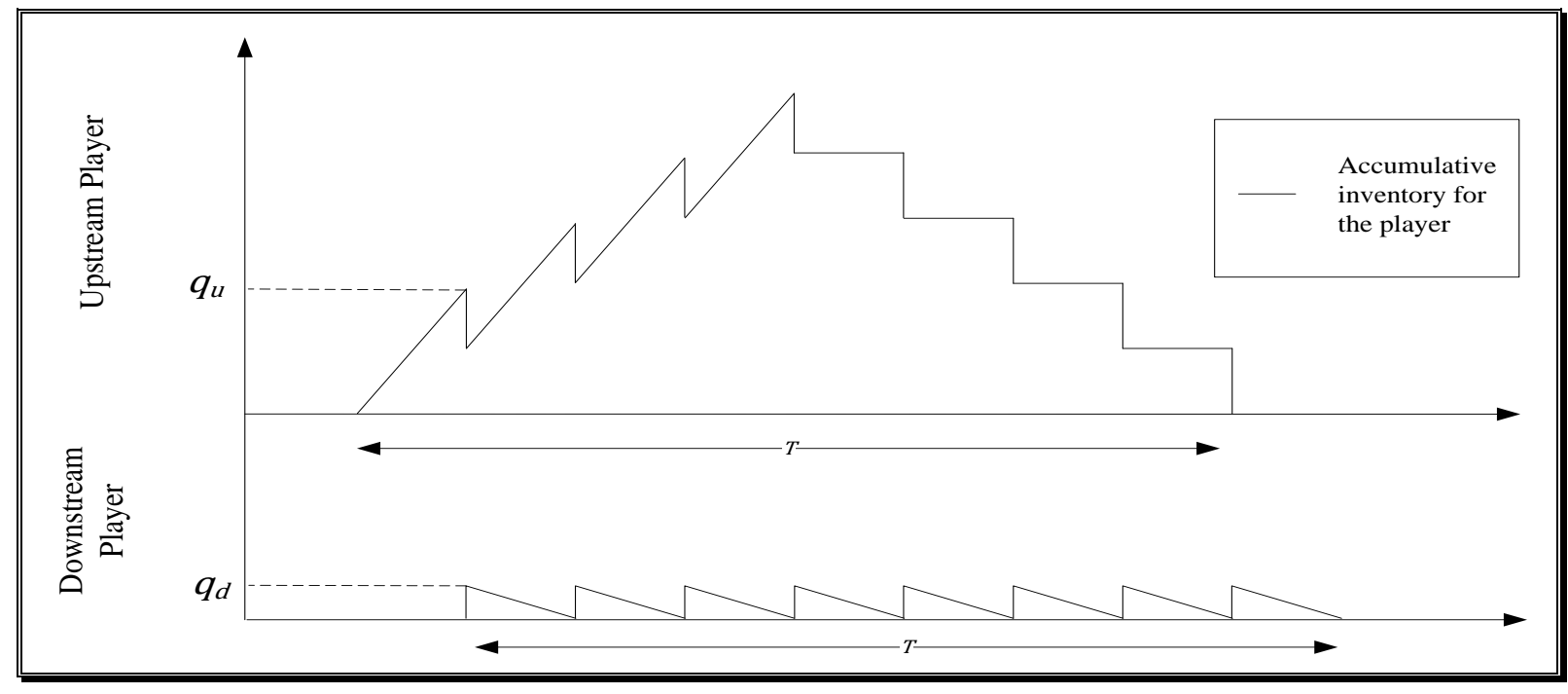

Figure 8-2. General traditional policy diagram 


\subsubsection{Consignment stock (CS)}

In Figure 8-3, Player $u$ produces $q_{u}$ units every $q_{u} / P$ units of time and ships it to Player $d$ during the production segment of its cycle $T$. When Player $u$ completes shipping $n q_{u}$ units, it stops its production for $T-n q_{u} / P$ unit of time. Player $d$ pays for items after they are withdrawn from inventory, in equal-sized payments at equal time intervals (Chapter 5). There are three cases of delay-in-payments. Player $d$ pays its owed balance to Player $u$ when it is invoiced, by the end of a permissible delay (interest-free) period, or past the delay period (interest is charged).

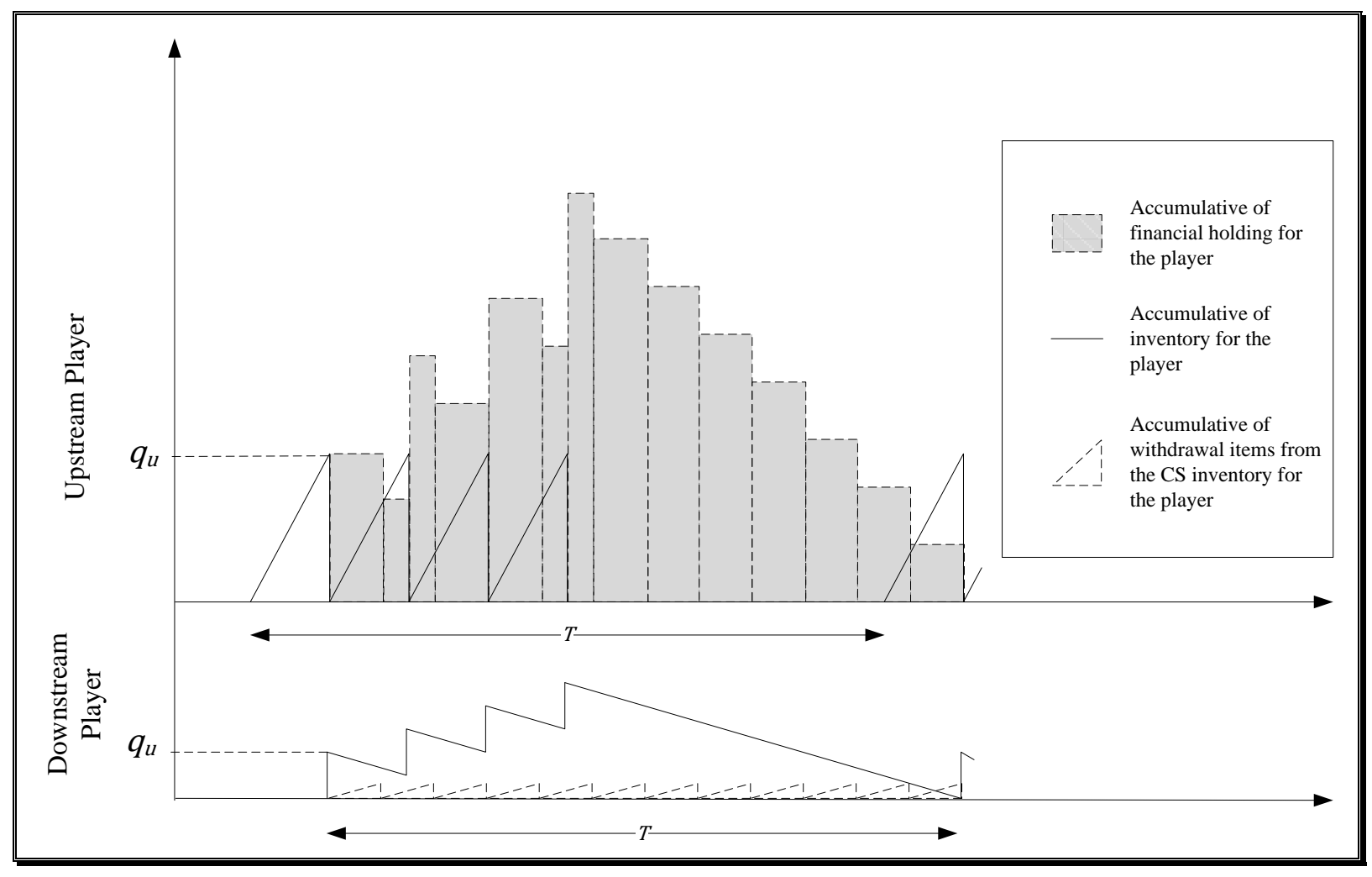

Figure 8-3. General consignment stock diagram

The above scenarios are investigated for the cases with and without the effects of delay-inpayments (interest-free or interest-charged). This requires developing 12 (4 scenarios $\times 3$ delay cases) models. To keep the chapter concise, a mathematical model that accounts for any of the three delay periods is developed for each scenario. To do so, the following parameters $\alpha_{1, j}, \alpha_{2, i}, \beta_{1, j}$ and $\beta_{2, i}$ are used in the mathematical modelling. In case no delay-in-payment is offered, $\alpha_{1, j}=\alpha_{2, i}=\beta_{1, j}=\beta_{2, i}=0$ in Eqs. (8-1) - (8-16). Player $d$ (downstream) pays Player $u$ (upstream) when it receives an invoice. Accordingly, Player $u$ incurs no additional costs from 
delaying the payments and Player $d$ generates no additional returns from investing its revenue over the delay period. For the case of interest-free delay-in-payments, $\alpha_{1, j}$ and $\alpha_{2, i}>0$ where $\beta_{1, j}=$ $\beta_{2, i}=0$ in Eqs. (8-1) - (8-16). Player $d$ is given some time after receiving an invoice from Player $u$ to settle its balance at no cost. Player $u$ incurs an opportunity cost for a delayed payment while Player $d$ generates profit from investing the payment over the delay period. In case Player $d$ pays Player $u$ beyond the interest-free period (Case 3), $\alpha_{1, j}, \alpha_{2, i}, \beta_{1, j}$ and $\beta_{2, i}>0$ in Eqs. (8-1) - (8-16). Player $d$ generates profit from investing the payment over the delay period and incurs additional cost as payment is made beyond the interest-free period. Player $u$ generates more revenue from charging Player $d$ interest on the outstanding payment for the extended period only.

\subsection{Scenario 1: No Consignment-No Consignment (NC-NC)}

This scenario assumes that the suppliers and the buyers adopt a TP with the vendor (see Figure 10-1 in Appendix 6). There are five terms in the sum of the suppliers' total annual profits, given in Eq. (8-1) below. The terms are (1) revenues less the costs of purchased components and production, (2) earnings from charging the vendor interest on payments past their permissible delay periods,

(3) setup costs, (4) storage and in-house financial-holding costs of parts, and (5) opportunity costs from not investing the money, the vendor owes the suppliers, over the permissible delay periods. The suppliers' total annual profit is:

$$
\begin{aligned}
T P_{s}^{N C-N C}=\sum_{j=1}^{x} & \sum_{i=1}^{y}\left(c_{s_{j}}^{s}-\gamma_{j} c_{s_{j}}^{p}-c_{s_{j}}^{p r}\right) \theta_{j} d_{i}+\frac{T}{P_{v}} \sum_{j=1}^{x} \sum_{i=1}^{y} c_{s_{j}}^{s} I_{s_{j}}^{c} \beta_{1, j}\left(1+\alpha_{1, j}\right) \frac{\theta_{j} d_{i}^{2}}{n_{1, j}} \\
& -\frac{1}{T} \sum_{j=1}^{x} S_{s_{j}}-\frac{T}{2 P_{v}} \sum_{j=1}^{x} \sum_{i=1}^{y}\left(h_{s_{j}}^{s}+h_{s_{j}}^{f_{1}}\right)\left(P_{s_{j}}-\theta_{j} P_{v}\right) \frac{\theta_{j} d_{i}^{2}}{P_{s_{j}}} \\
& -\frac{T}{P_{v}} \sum_{j=1}^{x} \sum_{i=1}^{y} h_{s_{j}}^{f_{2}}\left(\alpha_{1, j}+\beta_{1, j}\left(1+\alpha_{1, j}\right)\right) \frac{\theta_{j} d_{i}^{2}}{n_{1, j}}
\end{aligned}
$$

There are eight terms in the vendor's annual profit, given in Eq. (8-2) below. The terms are (1) revenue less the costs of purchased components and production, (2) earning from charging buyers interest on payments past their permissible delay periods, (3) the interest earned by the vendor from investing the money it owes to the suppliers, (4) sum of setup, ordering, and payment transactions costs, (5) storage and financial-holding cost of parts, (6) storage and in-house 
financial-holding costs of finished items, (7) opportunity cost of not investing the money, the buyers owe the vendor, over the permissible delay periods, and (8) sum of interest charged by the suppliers on payments past their permissible delay periods. The vendor's total annual profit per is:

$T P_{v}^{N C-N C}=\sum_{j=1}^{x} \sum_{i=1}^{y}\left(c_{v}^{s}-\theta_{j} c_{s_{j}}^{s}-c_{v}^{p r}\right) d_{i}+T c_{v}^{S} I_{v}^{c} \sum_{i=1}^{y} \beta_{2, i}\left(1+\alpha_{2, i}\right) \frac{d_{i}}{n_{2, i}}+$

$\frac{T I_{v}}{P_{v}} \sum_{j=1}^{x} \sum_{i=1}^{y} c_{S_{j}}^{S}\left(\alpha_{1, j}+\beta_{1, j}\left(1+\alpha_{1, j}\right)\right) \frac{\theta_{j} d_{i}^{2}}{n_{1, j}}-\frac{1}{T} \sum_{j=1}^{x}\left(S_{v}+n_{1, j} O_{v_{j}}+n_{1, j} c_{v_{j}}^{t}\right)-$

$\frac{T}{2 P_{v}} \sum_{j=1}^{x} \sum_{i=1}^{y}\left(h_{v_{j}}^{s, r}+h_{v_{j}}^{f, r}\right) \frac{\theta_{j} d_{i}^{2}}{n_{1, j}}-\frac{T}{2 P_{v}} \sum_{i=1}^{y}\left(h_{v}^{s, f}+h_{v}^{f_{1}, f}\right)\left(P_{v}-d_{i}\right) d_{i}-T h_{v}^{f_{2}, f} \sum_{i=1}^{y}\left(\alpha_{2, i}+\right.$

$\left.\beta_{2, i}\left(1+\alpha_{2, i}\right)\right) \frac{d_{i}}{n_{2, i}}-\frac{T}{P_{v}} \sum_{j=1}^{x} \sum_{i=1}^{y} c_{S_{j}}^{S} I_{S_{j}}^{c} \beta_{1, j}\left(1+\alpha_{1, j}\right) \frac{\theta_{j} d_{i}^{2}}{n_{1, j}}$

There are five terms in buyers' annual profit, given in Eq. (8-3) below. The terms are (1) revenue less the cost of purchased items, (2) earning from investing the money they owe to the vendor over the delay periods, (3) sum of ordering and payment transactions costs, (4) storage and financialholding costs of finished items, (5) interest charged by the vendor on payments past their permissible delay periods. The buyers' total annual profit is:

$$
\begin{aligned}
T P_{b}^{N C-N C}= & \sum_{i=1}^{y}\left(c_{b_{i}}^{s}-c_{v}^{s}\right) d_{i}+T c_{v}^{s} \sum_{i=1}^{y} I_{b_{i}}\left(\alpha_{2, i}+\beta_{2, i}\left(1+\alpha_{2, i}\right)\right) \frac{d_{i}}{n_{2, i}}-\frac{1}{T} \sum_{i=1}^{y}\left(O_{b_{i}}+c_{b_{i}}^{t}\right) n_{2, i} \\
& -\frac{T}{2} \sum_{i=1}^{y}\left(h_{b_{i}}^{s}+h_{b_{i}}^{f}\right) \frac{d_{i}}{n_{2, i}}-T c_{v}^{s} I_{v}^{c} \sum_{i=1}^{y} \beta_{2, i}\left(1+\alpha_{2, i}\right) \frac{d_{i}}{n_{2, i}}
\end{aligned}
$$

The system's total annual profit, $T P_{\text {system }}^{Z}=T P_{s}^{Z}+T P_{v}^{Z}+T P_{b}^{Z}$, where $z$ represents Scenarios 1 , 2, 3 and 4. $T P_{\text {system }}^{1}$ is concave since its second derivative is negative for $T=T_{N C-N C}>0$, and is given as:

$T_{N C-N C}=\sqrt{\frac{A_{N C-N C}}{B_{N C-N C}}}$

, where 
$A_{N C-N C}=\sum_{i=1}^{y} \sum_{j=1}^{x}\left(S_{s_{j}}+S_{v}+n_{1, j} O_{v_{j}}+n_{1, j} c_{v_{j}}^{t}+n_{2, i} O_{b_{i}}+n_{2, i} c_{b_{i}}^{t}\right)$

and

$B_{N C-N C}=\frac{1}{2 P_{v}} \sum_{j=1}^{x} \sum_{i=1}^{y}\left(h_{s_{j}}^{s}+h_{s_{j}}^{f_{1}}\right)\left(P_{s_{j}}-\theta_{j} P_{v}\right) \frac{\theta_{j} d_{i}^{2}}{P_{s_{j}}}-\frac{1}{P_{v}} \sum_{j=1}^{x} \sum_{i=1}^{y}\left(c_{s_{j}}^{s} I_{v}-h_{s_{j}}^{f_{2}}\right)\left(\alpha_{1, j}+\right.$

$\left.\beta_{1, j}\left(1+\alpha_{1, j}\right)\right) \frac{\theta_{j} d_{i}^{2}}{n_{1, j}}+\frac{1}{2 P_{v}} \sum_{j=1}^{x} \sum_{i=1}^{y}\left(h_{v_{j}}^{s, r}+h_{v_{j}}^{f, r}\right) \frac{\theta_{j} d_{i}^{2}}{n_{1, j}}+\frac{1}{2 P_{v}} \sum_{i=1}^{y}\left(h_{v}^{s, f}+h_{v}^{f_{1}, f}\right)\left(P_{v}-d_{i}\right) d_{i}-$

$\sum_{i=1}^{y}\left(c_{v}^{S} I_{b_{i}}-h_{v}^{f_{2}, f}\right)\left(\alpha_{2, i}+\beta_{2, i}\left(1+\alpha_{2, i}\right)\right) \frac{d_{i}}{n_{2, i}}+\frac{1}{2} \sum_{i=1}^{y}\left(h_{b_{i}}^{s}+h_{b_{i}}^{f}\right) \frac{d_{i}}{n_{2, i}}$

$B_{N C-N C}>0$ since $c_{s_{j}}^{S} I_{v}<h_{s_{j}}^{f_{2}}, P_{v}>d_{i}$, and $c_{v}^{S} I_{b_{i}}<h_{v}^{f_{2}, f}$.

\subsection{Scenario 2: No Consignment-Consignment (NC-C)}

This scenario assumes that the suppliers adopt TP agreements with the vendor who adopts CS agreements with its buyers (see Figure 10-2 in Appendix 6). The five terms in the suppliers' total annual profit in Eq. (8-5) below have the same or similar definitions as those in Eq. (8-1). The suppliers' total annual profit is:

$$
\begin{aligned}
T P_{S}^{N C-C}=\sum_{j=1}^{x} & \sum_{i=1}^{y}\left(c_{s_{j}}^{s}-\gamma_{j} c_{s_{j}}^{p}-c_{s_{j}}^{p r}\right) \theta_{j} d_{i}+\frac{T}{P_{v}} \sum_{j=1}^{x} \sum_{i=1}^{y} c_{s_{j}}^{s} I_{s_{j}}^{c} \beta_{1, j}\left(1+\alpha_{1, j}\right) \frac{\theta_{j} d_{i}^{2}}{n_{1, j}}-\frac{1}{T} \sum_{j=1}^{x} S_{s_{j}} \\
& -\frac{T}{2 P_{v}} \sum_{j=1}^{x} \sum_{i=1}^{y}\left(h_{s_{j}}^{s}+h_{s_{j}}^{f_{1}}\right)\left(P_{s_{j}}-\theta_{j} P_{v}\right) \frac{\theta_{j} d_{i}^{2}}{P_{s_{j}}} \\
& -\frac{T}{P_{v}} \sum_{j=1}^{x} \sum_{i=1}^{y} h_{s_{j}}^{f_{2}}\left(\alpha_{1, j}+\beta_{1, j}\left(1+\alpha_{1, j}\right)\right) \frac{\theta_{j} d_{i}^{2}}{n_{1, j}}
\end{aligned}
$$

The eight terms in the vendor's annual profit in Eq. (8-6) below have the same or similar definitions to those in Eq. (8-2). The vendor's annual profit is: 


$$
\begin{aligned}
& T P_{v}^{N C-C}=\sum_{i=1}^{y} \sum_{j=1}^{x}\left(c_{v}^{s}-\theta_{j} c_{s_{j}}^{s}-c_{v}^{p r}\right) d_{i}+T c_{v}^{S} I_{v}^{c} \sum_{i=1}^{y} \beta_{2, i}\left(1+\alpha_{2, i}\right) \frac{d_{i}}{m_{2, i}}+ \\
& \frac{T I_{v}}{P_{v}} \sum_{j=1}^{x} \sum_{i=1}^{y} c_{s_{j}}^{s}\left(\alpha_{1, j}+\beta_{1, j}\left(1+\alpha_{1, j}\right)\right) \frac{\theta_{j} d_{i}^{2}}{n_{1, j}}-\frac{1}{T} \sum_{J=1}^{X}\left(S_{v}+n_{1, j} O_{v_{j}}+n_{1, j} c_{v_{j}}^{t}\right)- \\
& \frac{T}{2 P_{v}} \sum_{j=1}^{x} \sum_{i=1}^{y}\left(h_{v_{j}}^{s, r}+h_{v_{j}}^{f, r}\right) \frac{\theta_{j} d_{i}^{2}}{n_{1, j}}-\frac{T}{2 P_{v}} \sum_{i=1}^{y}\left(h_{v}^{s, f}+h_{v}^{f_{1}, f}-\left(n_{2, i}-1\right) h_{v}^{f_{2}, f}\right) \frac{d_{i}^{2}}{n_{2, i}}- \\
& \frac{T h_{v}^{f_{2}, f}}{2} \sum_{i=1}^{y}\left(m_{2, i}+2 \alpha_{2, i}+2 \beta_{2, i}\left(1+\alpha_{2, i}\right)+1\right) \frac{d_{i}}{m_{2, i}}-\frac{T}{P_{v}} \sum_{j=1}^{x} \sum_{i=1}^{y} c_{s_{j}}^{s} I_{s_{j}}^{c} \beta_{1, j}\left(1+\alpha_{1, j}\right) \frac{\theta_{j} d_{i}^{2}}{n_{1, j}}
\end{aligned}
$$

The five terms in the buyers' annual profit in Eq. (8-7) below have the same or similar definitions as those in Eq. (8-3). The buyers incur no financial-holding costs because of adopting the CS agreement and their total annual profit is:

$$
\begin{gathered}
T P_{b}^{N C-C}=\sum_{i=1}^{y}\left(c_{b_{i}}^{s}-c_{v}^{s}\right) d_{i}+\frac{T}{2} \sum_{i=1}^{y} c_{b_{i}}^{s} I_{b_{i}}\left(2 \alpha_{2, i}+2 \beta_{2, i}\left(1+\alpha_{2, i}\right)+1\right) \frac{d_{i}}{m_{2, i}} \\
-\frac{1}{T} \sum_{i=1}^{y}\left(n_{2, i} O_{b_{i}}+m_{2, i} c_{b_{i}}^{t}\right)-\frac{T}{2} \sum_{i=1}^{y} h_{b_{i}}^{s}\left(d_{i}-\frac{\left(n_{2, i}-1\right) d_{i}^{2}}{n_{2, i} P_{v}}\right) \\
-T c_{v}^{s} I_{v}^{c} \sum_{i=1}^{y} \beta_{2, i}\left(1+\alpha_{2, i}\right) \frac{d_{i}}{m_{2, i}}
\end{gathered}
$$

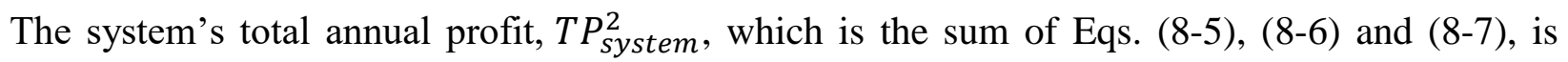
concave since its second derivative is negative for all values of $T=T_{N C-C}>0$, which is given as:

$T_{N C-C}=\sqrt{\frac{A_{N C-C}}{B_{N C-C}}}$

, where

$A_{N C-C}=\sum_{i=1}^{y} \sum_{j=1}^{x}\left(S_{s_{j}}+S_{v}+n_{1, j} O_{v_{j}}+n_{1, j} c_{v_{j}}^{t}+n_{2, i} O_{b_{i}}+m_{2, i} c_{b_{i}}^{t}\right)$

and 


$$
\begin{aligned}
& B_{N C-C}=\frac{1}{2 P_{v}} \sum_{j=1}^{x} \sum_{i=1}^{y}\left(h_{s_{j}}^{s}+h_{s_{j}}^{f_{1}}\right)\left(P_{s_{j}}-\theta_{j} P_{v}\right) \frac{\theta_{j} d_{i}^{2}}{P_{s_{j}}}-\frac{1}{P_{v}} \sum_{j=1}^{x} \sum_{i=1}^{y}\left(c_{s_{j}}^{s} I_{v}-h_{s_{j}}^{f_{2}}\right)\left(\alpha_{1, j}+\right. \\
& \left.\beta_{1, j}\left(1+\alpha_{1, j}\right)\right) \frac{\theta_{j} d_{i}^{2}}{n_{1, j}}+\frac{1}{2 P_{v}} \sum_{j=1}^{x} \sum_{i=1}^{y}\left(h_{v_{j}}^{s, r}+h_{v_{j}}^{f, r}\right) \frac{\theta_{j} d_{i}^{2}}{n_{1, j}}+\frac{1}{2 P_{v}} \sum_{i=1}^{y}\left(h_{v}^{s, f}+h_{v}^{f_{1}, f}-\left(n_{2, i}-\right.\right. \\
& \left.1) h_{v}^{f_{2}, f}-\left(n_{2, i}-1\right) h_{b_{i}}^{s}\right) \frac{d_{i}^{2}}{n_{2, i}}+\frac{1}{2} \sum_{i=1}^{y}\left(h_{v}^{f_{2}, f}+h_{b_{i}}^{s}\right) d_{i}-\frac{1}{2} \sum_{i=1}^{y}\left(c_{b_{i}}^{s} I_{b_{i}}-h_{v}^{f_{2}, f}\right)\left(2 \alpha_{2, i}+\right. \\
& \left.2 \beta_{2, i}\left(1+\alpha_{2, i}\right)+1\right) \frac{d_{i}}{m_{2, i}} \\
& B_{N C-C}>0 \text { since } P_{s_{j}}>\theta_{j} P_{v}, c_{s_{j}}^{s} I_{v}<h_{s_{j}}^{f_{2}}, h_{v}^{s, f}+h_{v}^{f_{1}, f}>\left(n_{2, i}-1\right) h_{v}^{f_{2}, f}+\left(n_{2, i}-1\right) h_{b_{i}}^{s}, \text { and } \\
& c_{b_{i}}^{s} I_{b_{i}}<h_{v}^{f_{2}, f} .
\end{aligned}
$$

\subsection{Scenario 3: Consignment-No Consignment (C-NC)}

This scenario assumes that the suppliers adopt CS agreements with the vendor who adopts TP agreements with the buyers (see Figure 10-3 in Appendix 6). The terms in Eq. (8-9) have the same or similar definitions to those in Eq. (8-1). The suppliers' total annual profit is

$$
\begin{aligned}
T P_{S}^{C-N C}=\sum_{j=1}^{x} & \sum_{i=1}^{y}\left(c_{s_{j}}^{s}-\gamma_{j} c_{s_{j}}^{p}-c_{s_{j}}^{p r}\right) \theta_{j} d_{i}+\frac{T}{P_{v}} \sum_{j=1}^{x} \sum_{i=1}^{y} c_{s_{j}}^{s} I_{s_{j}}^{c} \beta_{1, j}\left(1+\alpha_{1, j}\right) \frac{\theta_{j} d_{i}^{2}}{m_{1, j}}-\frac{1}{T} \sum_{j=1}^{x} S_{s_{j}} \\
& -\frac{T}{2} \sum_{j=1}^{x} \sum_{i=1}^{y}\left(h_{s_{j}}^{s}+h_{s_{j}}^{f_{1}}-\left(n_{1, j}-1\right) h_{s_{j}}^{f_{2}}\right) \frac{\theta_{j}^{2} d_{i}^{2}}{n_{1, j} P_{s_{j}}} \\
& -\frac{T}{2 P_{v}} \sum_{j=1}^{x} \sum_{i=1}^{y} h_{s_{j}}^{f_{2}}\left(m_{1, j}+2 \alpha_{1, j}+2 \beta_{1, j}\left(1+\alpha_{1, j}\right)+1\right) \frac{\theta_{j} d_{i}^{2}}{m_{1, j}}
\end{aligned}
$$

The terms in Eq. (8-10) below have the same or similar definitions to those in Eq. (8-2). The vendor incurs no financial-holding cost for parts stored at its warehouse as a result of adopting the CS agreement with the suppliers and its total annual profit is: 


$$
\begin{aligned}
& T P_{v}^{C-N C}=\sum_{i=1}^{y} \sum_{j=1}^{x}\left(c_{v}^{S}-\theta_{j} c_{s_{j}}^{S}-c_{v}^{p r}\right) d_{i}+T c_{v}^{S} I_{v}^{c} \sum_{i=1}^{y} \beta_{2, i}\left(1+\alpha_{2, i}\right) \frac{d_{i}}{n_{2, i}}+ \\
& \frac{T I_{v}}{2 P_{v}} \sum_{j=1}^{x} \sum_{i=1}^{y} c_{s_{j}}^{S}\left(2 \alpha_{1, j}+2 \beta_{1, j}\left(1+\alpha_{1, j}\right)+1\right) \frac{\theta_{j} d_{i}^{2}}{m_{1, j}}-\frac{1}{T} \sum_{j=1}^{x}\left(S_{v}+n_{1, j} O_{v_{j}}+m_{1, j} c_{v_{j}}^{t}\right)- \\
& \frac{T}{2 P_{v}} \sum_{i=1}^{y} \sum_{j=1}^{x} h_{v_{j}}^{s, r} \theta_{j}\left(d_{i}^{2}-\frac{\left(n_{1, j}-1\right) \theta_{j} P_{v}}{n_{1, j} P_{s_{j}}} d_{i}^{2}\right)-\frac{T}{2 P_{v}} \sum_{i=1}^{y}\left(h_{v}^{s, f}+h_{v}^{f_{1}, f}\right)\left(P_{v}-d_{i}\right) d_{i}- \\
& T h_{v}^{f_{2}, f} \sum_{i=1}^{y}\left(\alpha_{2, i}+\beta_{2, i}\left(1+\alpha_{2, i}\right)\right) \frac{d_{i}}{n_{2, i}}-\frac{T}{P_{v}} \sum_{j=1}^{x} \sum_{i=1}^{y} c_{s_{j}}^{s} I_{s_{j}}^{c} \beta_{1, j}\left(1+\alpha_{1, j}\right) \frac{\theta_{j} d_{i}^{2}}{m_{1, j}}
\end{aligned}
$$

The terms in Eq. (8-11) have the same or similar definitions to those in Eq. (8-3). The buyers' total annual profit is:

$$
\begin{gathered}
T P_{b}^{C-N C}=\sum_{i=1}^{y}\left(c_{b_{i}}^{s}-c_{v}^{s}\right) d_{i}+T c_{v}^{s} \sum_{i=1}^{y} I_{b_{i}}\left(\alpha_{2, i}+\beta_{2, i}\left(1+\alpha_{2, i}\right)\right) \frac{d_{i}}{n_{2, i}}-\frac{1}{T} \sum_{i=1}^{y}\left(O_{b_{i}}+c_{b_{i}}^{t}\right) n_{2, i} \\
-\frac{T}{2} \sum_{i=1}^{y}\left(h_{b_{i}}^{s}+h_{b_{i}}^{f}\right) \frac{d_{i}}{n_{2, i}}-T c_{v}^{s} I_{v}^{c} \sum_{i=1}^{y} \beta_{2, i}\left(1+\alpha_{2, i}\right) \frac{d_{i}}{n_{2, i}}
\end{gathered}
$$

The system's total annual profit is the sum of Eqs. (8-9), (8-10) and (8-11). It is concave since its second derivative is negative for $T=T_{C-N C}>0$, whose optimal value is:

$T_{C-N C}=\sqrt{\frac{A_{C-N C}}{B_{C-N C}}}$

, where

$A_{C-N C}=\sum_{i=1}^{y} \sum_{j=1}^{x}\left(S_{s_{j}}+S_{v}+n_{1, j} O_{v_{j}}+m_{1, j} c_{v_{j}}^{t}+n_{2, i} O_{b_{i}}+n_{2, i} c_{b_{i}}^{t}\right)$

and

$$
\begin{aligned}
& B_{C-N C}=\frac{1}{2} \sum_{j=1}^{x} \sum_{i=1}^{y}\left(h_{s_{j}}^{s}+h_{s_{j}}^{f_{1}}-\left(n_{1, j}-1\right) h_{s_{j}}^{f_{2}}-\left(n_{1, j}-1\right) h_{v_{j}}^{s, r}\right) \frac{\theta_{j}^{2} d_{i}^{2}}{n_{1, j} P_{s_{j}}}+ \\
& \frac{1}{2 P_{v}} \sum_{j=1}^{x} \sum_{i=1}^{y}\left(h_{s_{j}}^{f_{2}}+h_{v_{j}}^{s, r}\right) \theta_{j} d_{i}^{2}-\frac{1}{2 P_{v}} \sum_{j=1}^{x} \sum_{i=1}^{y}\left(c_{s_{j}}^{s} I_{v}-h_{s_{j}}^{f_{2}}\right)\left(2 \alpha_{1, j}+2 \beta_{1, j}\left(1+\alpha_{1, j}\right)+\right. \\
& \text { 1) } \frac{\theta_{j} d_{i}^{2}}{m_{1, j}}+\frac{1}{2 P_{v}} \sum_{i=1}^{y}\left(h_{v}^{s, f}+h_{v}^{f_{1}, f}\right)\left(P_{v}-d_{i}\right) d_{i}-\sum_{i=1}^{y}\left(c_{v}^{s} I_{b_{i}}-h_{v}^{f_{2}, f}\right)\left(\alpha_{2, i}+\beta_{2, i}(1+\right. \\
& \left.\left.\alpha_{2, i}\right)\right) \frac{d_{i}}{n_{2, i}}+\frac{1}{2} \sum_{i=1}^{y}\left(h_{b_{i}}^{s}+h_{b_{i}}^{f}\right) \frac{d_{i}}{n_{2, i}}
\end{aligned}
$$


$B_{C-N C}>0$ since $h_{s_{j}}^{s}+h_{s_{j}}^{f_{1}}>\left(n_{1, j}-1\right) h_{s_{j}}^{f_{2}}+\left(n_{1, j}-1\right) h_{v_{j}}^{s, r}, c_{s_{j}}^{s} I_{v}<h_{s_{j}}^{f_{2}}, P_{v}>d_{i}$, and $c_{v}^{s} I_{b_{i}}<$ $h_{v}^{f_{2}, f}$.

\subsection{Scenario 4: Consignment-Consignment (C-C)}

The last scenario assumes that the CS agreement exists between all players in the chain (see

Figure 10-4 in Appendix 6). The terms in Eq. (8-13) below have the same or similar definitions to those in Eq. (8-1). The suppliers' total annual profit is:

$$
\begin{aligned}
T P_{s_{j}}^{c-c}=\sum_{j=1}^{x} \sum_{i=1}^{y}\left(c_{s_{j}}^{s}-\gamma_{j} c_{s_{j}}^{p}-c_{s_{j}}^{p r}\right) \theta_{j} d_{i}+\frac{T}{P_{v}} \sum_{j=1}^{x} \sum_{i=1}^{y} c_{s_{j}}^{s} I_{s_{j}}^{c} \beta_{1, j}\left(1+\alpha_{1, j}\right) \frac{\theta_{j} d_{i}^{2}}{m_{1, j}}-\frac{1}{T} \sum_{j=1}^{x} S_{s_{j}} \\
-\frac{T}{2} \sum_{j=1}^{x} \sum_{i=1}^{y}\left(h_{s_{j}}^{s}+h_{s_{j}}^{f_{1}}-\left(n_{1, j}-1\right) h_{s_{j}}^{f_{2}}\right) \frac{\theta_{j}^{2} d_{i}^{2}}{n_{1, j} P_{s_{j}}} \\
-\frac{T}{2 P_{v}} \sum_{j=1}^{x} \sum_{i=1}^{y} h_{s_{j}}^{f_{2}}\left(m_{1, j}+2 \alpha_{1, j}+2 \beta_{1, j}\left(1+\alpha_{1, j}\right)+1\right) \frac{\theta_{j} d_{i}^{2}}{m_{1, j}}
\end{aligned}
$$

The terms of Eq. (8-14) have the same or similar definitions to those in Eq. (8-2). The vendor incurs no financial-holding cost for parts stored at its warehouse because of adopting the CS agreement with the suppliers and its total annual profit is:

$$
\begin{aligned}
& T P_{v}^{c-c}=\sum_{i=1}^{y} \sum_{j=1}^{x}\left(c_{v}^{s}-\theta_{j} c_{s_{j}}^{s}-c_{v}^{p r}\right) d_{i}+T c_{v}^{s} I_{v}^{c} \sum_{i=1}^{y} \beta_{2, i}\left(1+\alpha_{2, i}\right) \frac{d_{i}}{m_{2, i}}+ \\
& \frac{T I_{v}}{2 P_{v}} \sum_{j=1}^{x} \sum_{i=1}^{y} c_{s_{j}}^{s}\left(2 \alpha_{1, j}+2 \beta_{1, j}\left(1+\alpha_{1, j}\right)+1\right) \frac{\theta_{j} d_{i}^{2}}{m_{1, j}}-\frac{1}{T} \sum_{j=1}^{x}\left(S_{v}+n_{1, j} O_{v_{j}}+m_{1, j} c_{v_{j}}^{t}\right)- \\
& \frac{T}{2 P_{v}} \sum_{i=1}^{y} \sum_{j=1}^{x} h_{v_{j}}^{s, r} \theta_{j}\left(d_{i}^{2}-\left(n_{1, j}-1\right) \frac{\theta_{j} P_{v}}{n_{1, j} P_{s, j}} d_{i}^{2}\right)-\frac{T}{2 P_{v}} \sum_{i=1}^{y}\left(h_{v}^{s, f}+h_{v}^{f_{1}, f}-\left(n_{2, i}-\right.\right. \\
& \left.1) h_{v}^{f_{2}, f}\right) \frac{d_{i}^{2}}{n_{2, i}}-\frac{T h_{v}^{f_{2}, f}}{2} \sum_{i=1}^{y}\left(m_{2, i}+2 \alpha_{2, i}+2 \beta_{2, i}\left(1+\alpha_{2, i}\right)+1\right) \frac{d_{i}}{m_{2, i}}- \\
& \frac{T}{P_{v}} \sum_{j=1}^{x} \sum_{i=1}^{y} c_{s_{j}}^{s} I_{S_{j}}^{c} \beta_{1, j}\left(1+\alpha_{1, j}\right) \frac{\theta_{j} d_{i}^{2}}{m_{1, j}}
\end{aligned}
$$

The terms of Eq. (8-15) have the same or similar definitions to those in Eq. (8-3). The buyers incur no financial-holding costs because of adopting the CS agreement and their total annual profit is: 


$$
\begin{gathered}
T P_{b}^{c-c}=\sum_{i=1}^{y}\left(c_{b_{i}}^{s}-c_{v}^{s}\right) d_{i}+\frac{T}{2} \sum_{i=1}^{y} c_{b_{i}}^{s} I_{b_{i}}\left(2 \alpha_{2, i}+2 \beta_{2, i}\left(1+\alpha_{2, i}\right)+1\right) \frac{d_{i}}{m_{2, i}} \\
-\frac{1}{T} \sum_{i=1}^{y}\left(n_{2, i} O_{b_{i}}+m_{2, i} c_{b_{i}}^{t}\right)-\frac{T}{2} \sum_{i=1}^{y} h_{b_{i}}^{s}\left(d_{i}-\frac{\left(n_{2, i}-1\right)}{n_{2, i} P_{v}} d_{i}^{2}\right) \\
-T c_{v}^{S} I_{v}^{c} \sum_{i=1}^{y} \beta_{2, i}\left(1+\alpha_{2, i}\right) \frac{d_{i}}{m_{2, i}}
\end{gathered}
$$

The system's total annual profit is the summation of Eqs. (8-13), (8-14) and (8-15). It is concave since its second derivative is negative for $T=T_{C-C}>0$ whose optimal value is:

$T_{C-C}=\sqrt{\frac{A_{C-C}}{B_{C-C}}}$

, where

$A_{C-C}=\sum_{i=1}^{y} \sum_{j=1}^{x}\left(S_{s_{j}}+S_{v}+n_{1, j} O_{v_{j}}+m_{1, j} c_{v_{j}}^{t}+n_{2, i} O_{b_{i}}+m_{2, i} c_{b_{i}}^{t}\right)$

and

$B_{C-C}=\frac{1}{2} \sum_{j=1}^{x} \sum_{i=1}^{y}\left(h_{s_{j}}^{s}+h_{s_{j}}^{f_{1}}-\left(n_{1, j}-1\right) h_{s_{j}}^{f_{2}}-\left(n_{1, j}-1\right) h_{v_{j}}^{s, r}\right) \frac{\theta_{j}^{2} d_{i}^{2}}{n_{1, j} P_{s_{j}}}+$

$\frac{1}{2 P_{v}} \sum_{j=1}^{x} \sum_{i=1}^{y}\left(h_{s_{j}}^{f_{2}}+h_{v_{j}}^{s, r}\right) \theta_{j} d_{i}^{2}-\frac{1}{2 P_{v}} \sum_{j=1}^{x} \sum_{i=1}^{y}\left(c_{s_{j}}^{s} I_{v}-h_{s_{j}}^{f_{2}}\right)\left(2 \alpha_{1, j}+2 \beta_{1, j}\left(1+\alpha_{1, j}\right)+\right.$

1) $\frac{\theta_{j} d_{i}^{2}}{m_{1, j}}+\frac{1}{2 P_{v}} \sum_{i=1}^{y}\left(h_{v}^{s, f}+h_{v}^{f_{1}, f}-\left(n_{2, i}-1\right) h_{v}^{f_{2}, f}-\left(n_{2, i}-1\right) h_{b_{i}}^{s}\right) \frac{d_{i}^{2}}{n_{2, i}}+\frac{1}{2} \sum_{i=1}^{y}\left(h_{v}^{f_{2}, f}+\right.$

$\left.h_{b_{i}}^{S}\right) d_{i}-\frac{1}{2} \sum_{i=1}^{y}\left(c_{b_{i}}^{S} I_{b_{i}}-h_{v}^{f_{2}, f}\right)\left(2 \alpha_{2, i}+2 \beta_{2, i}\left(1+\alpha_{2, i}\right)+1\right) \frac{d_{i}}{m_{2, i}}$

$B_{C-C}>0$ since $h_{s_{j}}^{s}+h_{s_{j}}^{f_{1}}>\left(n_{1, j}-1\right) h_{s_{j}}^{f_{2}}+\left(n_{1, j}-1\right) h_{v_{j}}^{s, r}, c_{s_{j}}^{s} I_{v}<h_{s_{j}}^{f_{2}}, h_{v}^{s, f}+h_{v}^{f_{1}, f}>$ $\left(n_{2, i}-1\right) h_{v}^{f_{2}, f}+\left(n_{2, i}-1\right) h_{b_{i}}^{s}$, and $c_{b_{i}}^{s} I_{b_{i}}<h_{v}^{f_{2}, f}$.

\subsection{Solution Procedure}

The models developed in Sections 8.2 to 8.5 are optimized using a similar solution procedure of that described in Jaber and Goyal (2008). It determines the optimal number of shipments $\left(n_{1, j}\right.$ and $\left.n_{2, i}\right)$ and payments $\left(m_{1, j}\right.$ and $\left.m_{2, i}\right)$ and computes $T_{z}^{*}$ and $T P_{\text {system }}^{Z}$, where $z$ represents Scenarios 1,2, 3 and 4. The steps are followed: 
1. Input the value of the parameters for the suppliers, the vendor, and the buyers.

2. Set $\left(n_{1,1}, m_{1,1}, \ldots, n_{1, x}, m_{1, x}, n_{2,1}, m_{2,1}, \ldots, n_{2, y}, m_{2, y}\right)$ equal to 1 .

3. Calculate $T_{z}$ and $T P_{\text {system }}^{Z}$, set $T P_{\text {system }}^{Z}=$ Value A.

4. Increase $m_{2, y}$ by 1 (i.e. $m_{2, y}+1=2$ ). Calculate $T_{z}$ and $P_{\text {system }}^{z}$, set $T P_{\text {system }}^{z}=$ Value B.

5. Compare Value A with Value B

a. If Value $\mathrm{B}>$ Value $\mathrm{A}$, set Value $\mathrm{B}=$ Value $\mathrm{A}$, increase $m_{2, y}+1$ by 1 (i.e. $m_{2, y}=$ 3 ) and calculate $T_{z}$ and $T P_{\text {system }}^{Z}$ for the new combination of $n_{1,1}, \ldots, m_{2, y}+2$.

b. Set the new $T P_{\text {system }}^{Z}$ as Value B and compare this value with Value A.

c. Stop when $T P_{\text {system }}^{Z}$ starts to decrease (Value A > Value B) and go to the next step. Save Value A from this step for a later comparison.

6. Increase $n_{2, y}$ by 1 (i.e. $\left.1,1,1, \ldots, 2,1\right)$. Calculate $T_{z}$ and $T P_{\text {system }}^{Z}$ and set this value as Value B.

7. Compare Value A (Step 5.c.) with Value B (Step 6)

a. If Value $\mathrm{B}>$ Value $\mathrm{A}$, set Value $\mathrm{B}=$ Value $\mathrm{A}$,

b. Repeat the Steps $4-7 . a$.

c. Stop when $T P_{\text {system }}^{Z}$ starts to decrease (Value A > Value B) and go to the next step. Save Value A and the optimal values of the decision variables from this step for a later comparison.

8. Continue the search in a similar manner $\forall m_{2, i}$ and $n_{2, i}$, and $\forall m_{1, i}$ and $n_{1, i}$ until you reach $m_{1,1}$ and $n_{1,1}$.

9. Increase $n_{1,1}$ by 1 (i.e. $n_{1,1}=2$ ) and repeat the search. Stop if you got a lower total profit when $n_{1,1}=2$.

The combination of $\left(n_{1,1}, m_{1,1}, \ldots, n_{1, x}, m_{1, x}, n_{2,1}, m_{2,1}, \ldots, n_{2, y}, m_{2, y}\right)$ that return the highest system profit $T P_{\text {system }}^{Z}$ are the optimal solutions $\left(n_{1,1}^{*}, m_{1,1}^{*}, \ldots, n_{1, x}^{*}, m_{1, x}^{*}, n_{2,1}^{*}, m_{2,1}^{*}, \ldots, n_{2, y}^{*}, m_{2, y}^{*}\right)$ which its optimal cycle length is $T_{z}^{*}\left(n_{1,1}^{*}, m_{1,1}^{*}, \ldots, n_{1, x}^{*}, m_{1, x}^{*}, n_{2,1}^{*}, m_{2,1}^{*}, \ldots, n_{2, y}^{*}, m_{2, y}^{*}\right)$ where z represents Scenarios 1, 2, 3 and 4. Some conditions must be met when using the solution procedure, for instance, $m_{2, y} \geq m_{1,1}$ to guarantee that vendor will not run out of cash, $n_{2, y} \geq n_{1,1}$ to ensure that the vendor will not run out of parts for its production, $T \leq 1$ to reduce the cycle length to be 
less than a year, the values of the decision variables $\left(n_{1, x}, m_{1, x}, n_{2, y}\right.$ and $\left.m_{2, y} \geq 1\right)$ and integer, and $m_{1, x}=n_{1, x}$ and $m_{2, y}=n_{1, y}$ when TP is adopted between two adjacent players.

\subsection{Numerical Example}

This section provides numerical examples to compare the coordination scenarios in Section 3 by considering a three-level supply chain consisting of two suppliers, a vendor, and three buyers. The values of some input parameters are the same as in Jaber and Goyal (2008). The values of the other parameters were set within reason. Each scenario is solved for three cases: (1) no delay-inpayments, (2) a downstream player makes a payment by the end of the permissible delay period (interest-free), and (3) a downstream player pays past a permissible delay period (incurs interest charges). The following values are used for the suppliers.

\begin{tabular}{cccccccccccc}
\hline Supplier $j$ & $S_{s_{j}}$ & $P_{s_{j}}$ & $\gamma_{j}$ & $I_{s_{j}}$ & $I_{s_{j}}^{c}$ & $c_{s_{j}}^{p}$ & $c_{s_{j}}^{p r}$ & $c_{s_{j}}^{S}$ & $h_{s_{j}}^{s}$ & $h_{s_{j}}^{f_{1}}$ & $h_{s_{j}}^{f_{2}}$ \\
\hline 1 & 400 & 750,000 & 2 & 0.10 & 0.13 & 0.5 & 1.0 & 2.7 & 0.14 & 0.10 & 0.27 \\
2 & 300 & 700,000 & 2 & 0.12 & 0.15 & 1.5 & 0.9 & 5.3 & 0.26 & 0.11 & 0.63 \\
\hline
\end{tabular}

The selling price of a part for Supplier $j$ is calculated as $c_{s_{j}}^{s}=\left(\gamma_{j} c_{s_{j}}^{p}+c_{s_{j}}^{p r}\right)\left(1+a_{s_{j}}\right)$ where $a_{s_{j}}$ is the profit margin for Supplier $j$ and it is set to $35 \%$. The storage-holding cost for Supplier $j$ is $h_{s_{j}}^{s}=0.05 c_{s_{j}}^{s}$, the in-house financial-holding cost for a part in Supplier's $j$ warehouse is $h_{s_{j}}^{f_{1}}=$ $I_{s_{j}} c_{s_{j}}^{p r}$, and the out-house financial-holding cost (losing opportunity) for a part in the vendor's warehouse is $h_{s_{j}}^{f_{2}}=I_{s_{j}} c_{s_{j}}^{S}$. The following common values are used for the vendor.

\begin{tabular}{ccccccccc}
\hline$S_{v}$ & $P_{v}$ & $I_{v}$ & $I_{v}^{c}$ & $c_{v}^{p r}$ & $c_{v}^{S}$ & $h_{v}^{s, f}$ & $h_{v}^{f_{1}, f}$ & $h_{v}^{f_{2}, f}$ \\
\hline 200 & 600,000 & 0.10 & 0.12 & 0.80 & 11.8 & 0.59 & 0.08 & 1.18 \\
\hline
\end{tabular}

The following values, relate to Supplier $j$, are also used for the vendor.

\begin{tabular}{cccccc}
\hline Supplier $j$ & $\theta_{j}$ & $O_{v_{j}}$ & $h_{v_{j}}^{s, r}$ & $h_{v_{j}}^{f, r}$ & $c_{v_{j}}^{t}$ \\
\hline 1 & 1 & 15 & 0.14 & 0.27 & 0.50 \\
2 & 1 & 20 & 0.26 & 0.53 & 0.50 \\
\hline
\end{tabular}

The vendor's selling price for a finished item is $c_{v}^{s}=\left(\sum_{j=1}^{x} c_{s_{j}}^{s}+c_{v}^{p r}\right)\left(1+a_{v}\right)$ where $a_{v}$ is the vendor's profit margin which is also set to $35 \%$. The unit storage-holding cost of a part bought from Supplier $j$ is $h_{v_{j}}^{s, r}=0.05 c_{s_{j}}^{s}$ and its financial-holding cost is $h_{v_{j}}^{f, r}=I_{v} c_{s_{j}}^{s}$. For finished items, 
The unit storage-holding cost is $h_{v}^{s, f}=0.05 c_{v}^{s}$, the in-house financial-holding cost is $h_{v}^{f_{1}, f}=$ $I_{v} c_{v}^{p r}$, and the out-house financial-holding cost (losing opportunity) is $h_{v}^{f_{2}, f}=I_{v} c_{v}^{s}$. The following values are used for the buyers.

\begin{tabular}{cccccccc}
\hline Buyer $i$ & $d_{i}$ & $O_{b_{i}}$ & $I_{b_{i}}$ & $c_{b_{i}}^{t}$ & $c_{b_{i}}^{s}$ & $h_{b_{i}}^{s}$ & $h_{b_{i}}^{f}$ \\
\hline 1 & 100,000 & 30 & 0.10 & 0.5 & 16.0 & 0.80 & 1.19 \\
2 & 75,000 & 50 & 0.08 & 1.0 & 15.4 & 0.77 & 1.79 \\
3 & 50,000 & 70 & 0.12 & 0.7 & 16.6 & 0.83 & 2.38 \\
\hline
\end{tabular}

The selling price for Buyer $i$ is calculated as $c_{b_{i}}^{s}=c_{v}^{s}\left(1+a_{b_{i}}\right)$ where $a_{b_{i}}$ is its profit margin and it is set to 35\%,30\% and 40\% for Buyers 1, 2 and 3, respectively. The storage-holding cost for Buyer $i$ is $h_{b_{i}}^{s}=0.05 c_{b_{i}}^{s}$ and its financial-holding cost is $h_{b_{i}}^{f}=I_{b_{i}} c_{v}^{S}$.

The values needed for the three delay-in-payments cases.

\begin{tabular}{cll}
\hline Case & & \\
\hline 1 & $\alpha_{1,1}=\alpha_{1,2}=0$ & $\beta_{1,1}=\beta_{1,2}=0$ \\
& $\alpha_{2,1}=\alpha_{2,2}=\alpha_{2,3}=0$ & $\beta_{2,1}=\beta_{2,2}=\beta_{2,3}=0$ \\
2 & $\alpha_{1,1}=0.25, \alpha_{1,2}=0.3$ & $\beta_{1,1}=\beta_{1,2}=0$ \\
& $\alpha_{2,1}=0.1, \alpha_{2,2}=0.15, \alpha_{2,3}=0.2$ & $\beta_{2,1}=\beta_{2,2}=\beta_{2,3}=0$ \\
3 & $\alpha_{1,1}=0.25, \alpha_{1,2}=0.3$ & $\beta_{1,1}=0.1, \beta_{1,2}=0.15$ \\
& $\alpha_{2,1}=0.1, \alpha_{2,2}=0.15, \alpha_{2,3}=0.2$ & $\beta_{2,1}=0.1, \beta_{2,2}=0.2, \beta_{2,3}=0.1$ \\
\hline
\end{tabular}

The steps of the solution procedure in Section 8.6 were programmed in Visual Basic for Application (VBA) using nested loops. Table 8-1 summarizes the results of an example search for the optimal values of the decision variables that maximize the system profit. The bolded values correspond to the optimal solution and the highest system profit.

Table 8-1. Example of manual search for optimal results for Scenario NC-C (Case 1)

\begin{tabular}{|c|c|c|c|c|c|c|c|c|c|c|c|c|c|c|c|}
\hline$n_{11}$ & $m_{11}$ & $n_{1,2}$ & $m_{12}$ & $n_{21}$ & $m_{21}$ & $n_{2,2}$ & $m_{2,2}$ & $n_{2,3}$ & $m_{2,3}$ & $T^{*}$ & Total profit (\$) & Value A & & Value B & Optimal value \\
\hline 1 & 1 & 1 & 1 & 1 & 1 & 1 & 1 & 1 & 1 & 0.072 & $2,041,684.02$ & $2,041,684.02$ & & & \\
\hline 1 & 1 & 1 & 1 & 1 & 1 & 1 & 1 & 1 & 2 & 0.070 & $2,040,958.27$ & $2,041,684.02$ & $>$ & $2,040,958.27$ & $2,041,684.02$ \\
\hline 1 & 1 & 1 & 1 & 1 & 1 & 1 & 1 & 2 & 1 & 0.075 & $2,040,935.58$ & $2,041,684.02$ & $>$ & $2,040,935.58$ & $2,041,684.02$ \\
\hline 1 & 1 & 1 & 1 & 1 & 1 & 1 & 2 & 1 & 1 & 0.072 & $2,041,606.27$ & $2,041,684.02$ & $>$ & $2,041,606.27$ & $2,041,684.02$ \\
\hline 1 & 1 & 1 & 1 & 1 & 1 & 2 & 1 & 1 & 1 & 0.075 & $2,041,453.95$ & $2,041,684.02$ & $>$ & $2,041,453.95$ & $2,041,684.02$ \\
\hline 1 & 1 & 1 & 1 & 1 & 2 & 1 & 1 & 1 & 1 & 0.070 & $2,040,940.30$ & $2,041,684.02$ & $>$ & $2,040,940.30$ & $2,041,684.02$ \\
\hline 1 & 1 & 1 & 1 & 2 & 1 & 1 & 1 & 1 & 1 & 0.075 & $2,042,088.70$ & $2,041,684.02$ & $<$ & $2,042,088.70$ & $2,042,088.70$ \\
\hline 1 & 1 & 1 & 1 & 2 & 1 & 1 & 1 & 1 & 2 & 0.073 & $2,041,334.09$ & $2,042,088.70$ & $>$ & $2,041,334.09$ & $2,042,088.70$ \\
\hline 1 & 1 & 1 & 1 & 2 & 1 & 1 & 1 & 2 & 1 & 0.078 & $2,041,386.54$ & $2,042,088.70$ & $>$ & $2,041,386.54$ & $2,042,088.70$ \\
\hline 1 & 1 & 1 & 1 & 2 & 1 & 1 & 2 & 1 & 1 & 0.075 & $2,042,008.85$ & $2,042,088.70$ & $>$ & $2,042,008.85$ & $2,042,088.70$ \\
\hline 1 & 1 & 1 & 1 & 2 & 1 & 2 & 1 & 1 & 1 & 0.078 & $2,041,904.66$ & $2,042,088.70$ & $>$ & $2,041,904.66$ & $2,042,088.70$ \\
\hline 1 & 1 & 1 & 1 & 2 & 2 & 1 & 1 & 1 & 1 & 0.073 & $2,041,315.18$ & $2,042,088.70$ & $>$ & $2,041,315.18$ & $2,042,088.70$ \\
\hline 1 & 1 & 1 & 1 & 3 & 1 & 1 & 1 & 1 & 1 & 0.077 & $2,041,972.91$ & $2,042,088.70$ & $>$ & $2,041,972.91$ & $2,042,088.70$ \\
\hline 2 & 2 & 2 & 2 & 2 & 2 & 2 & 2 & 2 & 2 & 0.079 & $2,039,783.65$ & $2,042,088.70$ & $>$ & $2,039,783.65$ & $2,042,088.70$ \\
\hline
\end{tabular}


The results in Table 8-2 show that an NC-C coordination policy gives the highest system profit for the three cases (with and without delay-in-payments). Although Case 2 (vendor-buyers follow CS) shows that offering the downstream players an interest-free permissible delay period increases the system profit, Case 3 returns the highest system profit of the three cases. In Case 3, upstream and downstream players make additional money. The upstream by charging interest on delayed payments and the downstream by investing them, whereas in Case 2 the upstream player does not earn interest. The results also show that the differences in profits among the Scenarios (NC-NC, NC-C, C-NC, and C-C) for all cases are insignificant. This suggests that the supply chain has some flexibility when it comes to choosing coordination contracts. The C-NC Scenario performed less than the other three because of the too many transactions (payments and shipments). The NC-C Scenario is a practical scenario since it has the least number of transactions and the numbers are somewhat uniform (easy to implement).

Table 8-2. The optimal results for Cases 1, 2 and 3

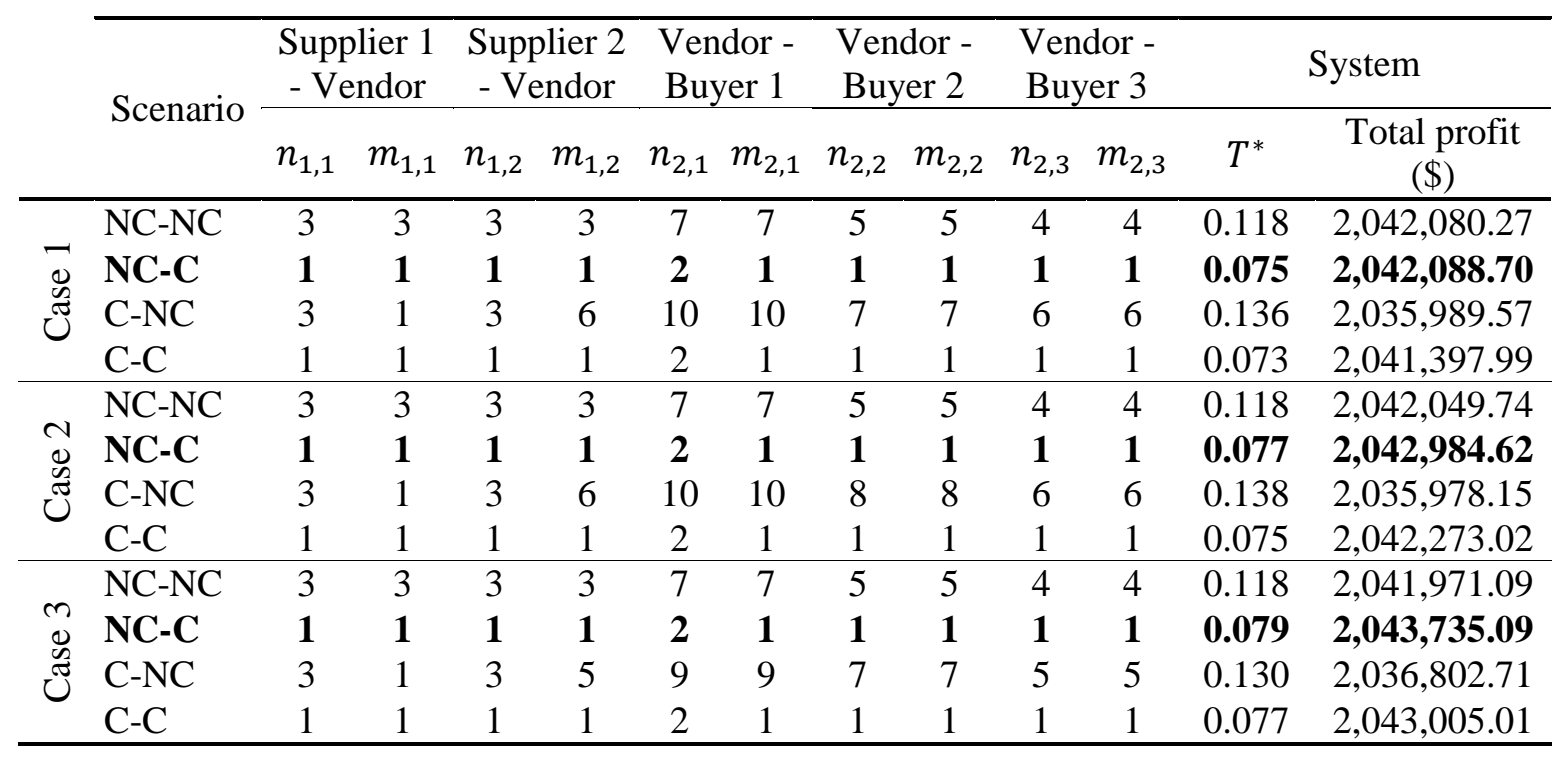

Table 8-3 (next page) shows the total profit for each player in the system for the four scenarios and the three cases of Table 8-2. The scenario that yields the highest system profit is not necessarily the best for all players. Table 8-3 shows that the suppliers and the buyers prefer NC (TP) and CS agreements with the vendor, respectively, who prefers otherwise. The vendor saves the financialholding cost when it has a CS agreement with the suppliers as it pays after withdrawing the consigned parts and earns by investing the money it owes to a supplier for a period between receiving an invoice and paying its amount. In a CS agreement, the vendor incurs an out-of-house 
financial-holding cost when finished items are in the buyers' facilities. The results also show that offering the buyers delay-in-payments without charging them interest on the outstanding payments reduces the vendor's profit more than it does when charging interest on payments past their delay periods. Table 8-3 shows that it is always better for the buyers to adopt a CS agreement with the vendor. This is obvious as they can invest their money before paying the vendor.

Table 8-3. Suppliers', Vendor's and Buyers' total profit for the optimal results for Cases 1, 2 and 3

\begin{tabular}{|c|c|c|c|c|c|c|c|}
\hline & Scenario & $\begin{array}{c}\text { Supplier 1 } \\
\text { Total profit } \\
(\$)\end{array}$ & $\begin{array}{c}\text { Supplier } 2 \\
\text { Total profit } \\
(\$)\end{array}$ & $\begin{array}{l}\text { Vendor } \\
\text { Total profit } \\
(\$)\end{array}$ & $\begin{array}{c}\text { Buyer } 1 \\
\text { Total profit } \\
(\$)\end{array}$ & $\begin{array}{c}\text { Buyer } 2 \\
\text { Total profit } \\
(\$)\end{array}$ & $\begin{array}{c}\text { Buyer } 3 \\
\text { Total profit } \\
(\$)\end{array}$ \\
\hline \multirow{4}{*}{$\begin{array}{l}\bar{d} \\
\tilde{U} \\
\tilde{E}\end{array}$} & NC-NC & $154,025.52$ & $304,487.45$ & $679,206.40$ & $410,659.61$ & $261,810.29$ & $231,891.01$ \\
\hline & $\mathrm{NC}-\mathrm{C}$ & $152,113.51$ & $303,064.99$ & $665,594.41$ & $416,579.98$ & $266,852.36$ & $237,883.45$ \\
\hline & C-NC & $153,610.78$ & $303,925.15$ & $679,470.94$ & $409,198.54$ & $259,884.80$ & $229,899.37$ \\
\hline & $\mathrm{C}-\mathrm{C}$ & $151,237.19$ & $301,281.05$ & $667,772.08$ & $416,487.93$ & $266,807.28$ & $237,812.46$ \\
\hline \multirow{4}{*}{$\begin{array}{l}\text { v } \\
0 \\
\text { है } \\
\text { Uి }\end{array}$} & NC-NC & $153,942.03$ & $304,260.02$ & $678,618.30$ & $410,858.68$ & $262,061.39$ & $232,309.31$ \\
\hline & $\mathrm{NC}-\mathrm{C}$ & $152,114.44$ & $302,740.57$ & $662,690.23$ & $417,939.79$ & $267,983.43$ & 239,516.16 \\
\hline & C-NC & $153,366.76$ & $303,816.14$ & $679,075.23$ & $409,352.58$ & $260,151.76$ & $230,215.68$ \\
\hline & $\mathrm{C}-\mathrm{C}$ & $151,216.01$ & $300,914.61$ & $665,019.31$ & $417,810.66$ & $267,909.42$ & $239,403.01$ \\
\hline \multirow{4}{*}{$\begin{array}{l}n \\
\dot{w} \\
\tilde{E} \\
\tilde{E}\end{array}$} & NC-NC & $153,945.51$ & $304,290.61$ & $678,743.75$ & $410,814.07$ & $261,869.05$ & $232,308.10$ \\
\hline & $\mathrm{NC}-\mathrm{C}$ & $152,267.28$ & $302,901.66$ & $662,612.79$ & $418,236.98$ & $267,805.38$ & 239,910.99 \\
\hline & C-NC & $153,294.32$ & $303,702.84$ & $679,504.58$ & $409,519.77$ & $260,426.49$ & $230,354.70$ \\
\hline & $\mathrm{C}-\mathrm{C}$ & $151,345.68$ & $301,028.19$ & $665,020.44$ & $418,094.65$ & $267,732.88$ & $239,783.17$ \\
\hline
\end{tabular}

Table 8-4 (next page) shows the optimal quantities and the number of shipments for the suppliers and the buyers for the four scenarios and the three cases of Table 8-2. The results show that the optimal shipments from the suppliers to the vendor are equal to the summation of the optimal quantities of the buyers. Furthermore, they show that the optimal lot sizes $\left(n_{2, i}^{*} q_{2, i}^{*}\right)$ decrease for the buyers when adopting CS agreements and increase otherwise. Delay-in-payments increase (decrease) the buyers' order quantities when they have CS (TP) agreements with the vendor. Charging the buyers interest on outstanding payments also decreases their order quantities.

The numerical examples compared between the developed scenarios for a given set of parameters. Scenario NC-C with Case 3, payments made past their permissible delay periods, returned the highest profit. A sensitivity analysis is provided in the next section to investigate the effects of the input parameters on the four scenarios. 
Table 8-4. Optimal quantities and shipments for the optimal results Cases 1, 2 and 3

\begin{tabular}{|c|c|c|c|c|c|c|c|c|c|c|c|}
\hline & & Supplier 1 & & Supplier 2 & & Buyer 1 & & Buyer 2 & & Buyer 3 & \\
\hline & & $n_{1,1}$ & $q_{1,1}$ & $n_{1,2}$ & $q_{1,2}$ & $n_{2,1}$ & $q_{2,1}$ & $n_{2,2}$ & $q_{2,2}$ & $n_{2,3}$ & $q_{2,3}$ \\
\hline \multirow{4}{*}{$\begin{array}{l}\overline{0} \\
\tilde{J}\end{array}$} & NC-NC & 3 & 8,848 & 3 & 8,848 & 7 & 1,685 & 5 & 1,770 & 4 & 1,475 \\
\hline & NC-C & 1 & 16,875 & 1 & 16,875 & 2 & 3,750 & 1 & 5,625 & 1 & 3,750 \\
\hline & $\mathrm{C}-\mathrm{NC}$ & 3 & 10,170 & 3 & 10,170 & 10 & 1,356 & 7 & 1,453 & 6 & 1,130 \\
\hline & C-C & 1 & 16,493 & 1 & 16,493 & 2 & 3,665 & 1 & 5,498 & 1 & 3,665 \\
\hline \multirow{4}{*}{$\begin{array}{l}\text { D } \\
\text { J } \\
\tilde{J}\end{array}$} & NC-NC & 3 & 8,839 & 3 & 8,839 & 7 & 1,684 & 5 & 1,768 & 4 & 1,473 \\
\hline & $\mathrm{NC}-\mathrm{C}$ & 1 & 17,398 & 1 & 17,398 & 2 & 3,866 & 1 & 5,799 & 1 & 3,866 \\
\hline & $\mathrm{C}-\mathrm{NC}$ & 3 & 10,367 & 3 & 10,367 & 10 & 1,382 & 8 & 1,296 & 6 & 1,152 \\
\hline & $\mathrm{C}-\mathrm{C}$ & 1 & 16,980 & 1 & 16,980 & 2 & 3,773 & 1 & 5,660 & 1 & 3,773 \\
\hline \multirow{4}{*}{$\begin{array}{l}0 \\
0 \\
\tilde{J}\end{array}$} & NC-NC & 3 & 8,815 & 3 & 8,815 & 7 & 1,679 & 5 & 1,763 & 4 & 1,469 \\
\hline & NC-C & 1 & 17,861 & 1 & 17,861 & 2 & 3,969 & 1 & 5,954 & 1 & 3,969 \\
\hline & C-NC & 3 & 9,736 & 3 & 9,736 & 9 & 1,442 & 7 & 1,391 & 5 & 1,298 \\
\hline & C-C & 1 & 17,410 & 1 & 17,410 & 2 & 3,869 & 1 & 5,803 & 1 & 3,869 \\
\hline
\end{tabular}

\subsection{Sensitivity Analysis}

This section provides a sensitivity analysis or expands upon the results of Section 8.7. The values of the highest profits in Table 8-5 for the scenarios and the three cases of delay-in-payments are in bold. As the players at the same level (i.e. suppliers or buyers) have the same type of contract (TP or CS) with the vendor, for simplicity, the behavior of the models is tested for the ratio of the sum of the buyers' demand rates to the production rate of the vendor; e.g., $\left(\sum_{i=1}^{3} d_{i} / P_{v}\right)=a$ is calculated as $\left(d_{i} / \sum_{i=1}^{3} d_{i}\right) \cdot a \cdot P_{v}$. We do the same for other costs and interest rates; i.e., the holding cost is calculated as $\left(h_{v_{j}}^{s, r} / \sum_{j=1}^{2} h_{v_{j}}^{s, r}\right) \cdot a \cdot \sum_{j=1}^{2} h_{s_{j}}^{s}$. The player who suffers a loss because of coordination is compensated from the additional profits and savings generated.

The results in Table 8-5 show that as $\sum_{i=1}^{3} d_{i} / P_{v}$ increases, the system profit increases for the four scenarios in the three cases. As $\sum_{i=1}^{3} d_{i} / P_{v}$ approaches 1 , Scenario NC-NC with no delay-inpayments (Case 1) returns the highest profit. Scenario NC-C with extended delay-in-payments (Case 3) returns the highest profit for the three ratios of $\sum_{i=1}^{3} d_{i} / P_{v}$.

The results of varying $\sum_{j=1}^{2} S_{v} / S_{s_{j}}$, as seen in Table $8-5$, show that when $\sum_{j=1}^{2} S_{v} / S_{s_{j}}<1$ $\left(\sum_{j=1}^{2} S_{v} / S_{s_{j}} \geq 1\right)$, Scenario NC-C (NC-NC) returns the highest profit for the all cases (except for Case 3). NC-C performed better than NC-NC when the ratio is 1 , but not when it is 2 . The difference in profit between the two scenarios in Case 3 is insignificant when the ratio is 1 . The results show that the system profit decreases as $\sum_{j=1}^{2} S_{v} / S_{s_{j}}$ increases. This suggests that it is always better to reduce the setup cost of the vendor, which can be achieved by receiving semi- 
finished products from its suppliers instead of raw materials. The buyers favor CS when the vendor setup is lower than its suppliers and TP otherwise except for Case 3 (greater than its suppliers).

The results of varying $\sum_{i=1}^{3} \sum_{j=1}^{2} O_{b_{i}} / O_{v_{j}}$, as illustrated in Table 8-5, show that Scenario NC-NC returns the highest profit for Case 1 for all values of the ratio and for Cases 2 and 3 when the ratio $<3.5$. The difference in profit between NC-C and NC-NC is minimal when the ratio is 3.5. This suggests that it may not worth shifting to $\mathrm{NC}-\mathrm{C}$ from $\mathrm{NC}-\mathrm{NC}$ unless there are other intangible benefits. The results show that the system profit is highest when the ratio is low.

The fourth and the fifth analysis were performed to test the effect of varying $\sum_{i=1}^{3} h_{b_{i}}^{s} / h_{v}^{s, f}$ and $\sum_{j=1}^{2} h_{v_{j}}^{s, r} / h_{s_{j}}^{s}$. The results show that as $\sum_{i=1}^{3} h_{b_{i}}^{s} / h_{v}^{s, f}$ or $\sum_{j=1}^{2} h_{v_{j}}^{s, r} / h_{s_{j}}^{s}$ increases, the system profit decreases for the four scenarios in all three cases. The system profit is noticeably affected by the buyers' storage-holding costs. In Table 8-5, Scenario NC-C returns the highest system profit for all ratios of $\sum_{i=1}^{3} h_{b_{i}}^{s} / h_{v}^{s, f}$ and $\sum_{j=1}^{2} h_{v_{j}}^{s, r} / h_{s_{j}}^{s}$ in the cases of delay-in-payments. Two-level supply chain studies showed that adopting the best policy (TP or CS) depends on the values of the storageholding costs of the players. This finding seems not to hold in a complex supply chain structure like the one in this chapter.

The study of varying $\sum_{i=1}^{3} I_{b_{i}} / I_{v}^{c}$, in Table 8-5, demonstrates that Scenario NC-NC returns the highest profit for all cases when the ratio $\leq 2$. Scenario NC-C returns the highest profit when the ratio $>2$. CS is recommended between the buyers and the vendor when the ratio is considerably large. Extended delay periods of payments increase supply chain profitability. C-C is also as good since the difference in profits is negligible. There could be nonmonetary benefits of $\mathrm{C}-\mathrm{C}$ that may make the players agree to CS agreements among all.

The results of varying $\sum_{j=1}^{2} I_{v} / I_{s_{j}}^{c}$, as seen in Table 8-5, indicate that the vendor favors CS policy with its suppliers when its return on investment is considerably larger than the sum of the interests the suppliers charge the vendor (ratios 1.5 and 2.5) in Cases 2 and 3. This result is expected when the vendor can invest the balances it owes at rates that exceed the borrowing cost. Lower investments rates (ratio 0.5) do not tempt the vendor to go for CS agreements with its suppliers as shown in the three cases of delay in payments. 
Table 8-5. Results of the sensitivity analysis

\begin{tabular}{|c|c|c|c|c|c|c|c|c|c|}
\hline & Case 1 & & & Case 2 & & & Case 3 & & \\
\hline$\sum_{i=1} d_{i} / P_{v}$ & 0.10 & 0.50 & 0.90 & 0.10 & 0.50 & 0.90 & 0.10 & 0.50 & 0.90 \\
\hline NC-NC & 537,126 & $2,728,224$ & $4,927,267$ & 537,113 & 537,113 & 537,113 & 537,078 & 537,078 & 537,078 \\
\hline $\mathrm{NC}-\mathrm{C}$ & 537,739 & $2,728,148$ & $4,926,804$ & 538,232 & 538,232 & 538,232 & 538,646 & 538,646 & 538,646 \\
\hline $\mathrm{C}-\mathrm{NC}$ & 4,113 & $2,721,045$ & $4,917,150$ & 534,107 & 534,107 & 534,107 & 534,541 & 534,541 & 534,541 \\
\hline $\mathrm{C}-\mathrm{C}$ & 537,647 & $2,727,523$ & $4,925,553$ & 538,143 & 538,143 & 538,143 & 538,559 & 538,559 & 538,559 \\
\hline$\sum_{j=1}^{2} S_{v} / S_{S_{j}}$ & 0.10 & 1.00 & 2.00 & 0.10 & 1.00 & 2.00 & 0.10 & 1.00 & 2.00 \\
\hline NC-NC & $2,043,204$ & $2,038,251$ & $2,033,908$ & $2,043,179$ & $2,038,210$ & $2,033,876$ & $2,043,105$ & $2,038,124$ & $2,033,800$ \\
\hline $\mathrm{NC}-\mathrm{C}$ & $2,043,819$ & $2,036,579$ & $2,030,097$ & $2,044,939$ & $2,037,802$ & $2,031,589$ & $2,045,701$ & $2,038,829$ & $2,032,833$ \\
\hline $\mathrm{C}-\mathrm{NC}$ & $2,037,129$ & $2,032,124$ & $2,027,795$ & $2,037,146$ & $2,032,110$ & $2,027,784$ & $2,037,979$ & $2,032,977$ & $2,028,597$ \\
\hline $\mathrm{C}-\mathrm{C}$ & $2,043,647$ & $2,036,117$ & $2,029,630$ & $2,044,570$ & $2,037,383$ & $2,031,130$ & $2,045,338$ & $2,038,438$ & $2,032,378$ \\
\hline$\sum_{\substack{i=1 \\
/ O_{v_{j}}}}^{3} \sum_{j=1}^{2} O_{b_{i}}$ & 0.5 & 2.0 & 3.5 & 0.5 & 2.0 & 3.5 & 0.5 & 2.0 & 3.5 \\
\hline NC-NC & $2,050,189$ & $2,046,020$ & $2,043,254$ & $2,050,154$ & $2,045,986$ & $2,043,236$ & $2,050,111$ & 2,0 & $2,043,175$ \\
\hline $\mathrm{NC}-\mathrm{C}$ & $2,045,361$ & $2,043,667$ & 2,0 & $2,046,323$ & $2,044,800$ & & $2,047,138$ & &, 541 \\
\hline C-NC & $2,047,775$ & $2,041,750$ & $2,037,736$ & $2,047,760$ & $2,041,738$ & $2,037,718$ & $2,048,039$ & $2,042,301$ & $2,038,499$ \\
\hline $\mathrm{C}-\mathrm{C}$ & $2,044,961$ & $2,043,437$ & $2,042,296$ & $2,045,932$ & $2,044,409$ & $2,043,306$ & $2,046,752$ & $2,045,227$ & $2,044,143$ \\
\hline$\sum_{i=1} h_{b_{i}}^{s} / h_{v}^{s, f}$ & 0.5 & 2.0 & 3.5 & 0.5 & 2.0 & 3.5 & 0.5 & 2.0 & 3.5 \\
\hline NC-NC & $2,043,922$ & 2,04 & 2,0 & $2,043,900$ & 2,0 & 2,0 & $2,043,810$ & 007 & $2,042,237$ \\
\hline NC-C & $2,048,955$ & 2,04 & 2,04 & $2,050,356$ & 2,0 & & $2,051,568$ & 26 & 088 \\
\hline $\mathrm{C}-\mathrm{NC}$ & $2,038,750$ & 2,037 & 2,036 & $2,038,738$ & 2,0 & 2,0 & $2,039,718$ & 441 & $2,037,223$ \\
\hline $\mathrm{C}-\mathrm{C}$ & $2,048,500$ & $2,045,491$ & $2,042,833$ & $2,049,839$ & $2,046,608$ & $2,043,848$ & $2,051,119$ & $2,047,637$ & $2,044,693$ \\
\hline$\sum_{j=1}^{2} h_{v_{j}}^{s, r} / h_{s_{j}}^{s}$ & 0.5 & 2.0 & 3.5 & 0.5 & 2.0 & 3.5 & 0.5 & 2.0 & 3.5 \\
\hline NC-NC & $2,042,212$ & $2,041,845$ & $2,041,509$ & $2,042,168$ & 2,04 & & $2,042,089$ & & $2,041,415$ \\
\hline NC-C & $2,042,426$ & $2,042,074$ & $2,041,727$ & $2,043,400$ & $\mathbf{2 , 0 4 3 , 0 3 7}$ & 2,0 & $2,044,213$ & $2,043,839$ & $2,043,511$ \\
\hline C-NC & & & & & & & & & 5,110 \\
\hline $\mathrm{C}-\mathrm{C}$ & $2,042,043$ & $2,041,640$ & $2,041,253$ & $2,042,918$ & $2,042,612$ & $2,042,242$ & $2,043,848$ & $2,043,419$ & $2,043,064$ \\
\hline$\sum_{i=1}^{3} I_{b_{i}} / I_{v}^{c}$ & 0.5 & 2.0 & 3.5 & 0.5 & 2.0 & 3.5 & 0.5 & 2.0 & 3.5 \\
\hline NC-NC & 30 & 2,0 & 2,0 & 2,0 & 2,0 & & 52 & & 629 \\
\hline $\mathrm{NC}-\mathrm{C}$ & 282 & 2,04 & 2 & 2,0 & 2,0 & & 982 & 73 &, 612 \\
\hline C-NC & $2,035,990$ & $2,035,990$ & $2,035,990$ & $2,035,529$ & $2,035,869$ & $2,036,200$ & $2,035,868$ & $2,036,568$ & $2,037,293$ \\
\hline $\mathrm{C}-\mathrm{C}$ & $2,037,919$ & $2,039,688$ & $2,048,201$ & $2,037,780$ & $2,040,012$ & $2,051,995$ & $2,037,651$ & $2,040,226$ & $2,057,016$ \\
\hline$\sum_{j=1}^{2} I_{v} / I_{s_{j}}^{c}$ & 0.5 & 1.5 & 2.5 & 0.5 & 1.5 & 2.5 & 0.5 & 1.5 & 2.5 \\
\hline NC-NC & $2,041,528$ & $2,038,155$ & $2,035,376$ & $2,041,266$ & $2,036,260$ & 2,03 & $2,040,903$ & $2,034,360$ & $2,028,918$ \\
\hline NC-C & $2,035,768$ & $2,014,780$ & $2,000,076$ & $2,035,996$ & $2,015,104$ & 2,000 & $2,036,105$ & $2,015,378$ & $2,001,110$ \\
\hline $\mathrm{C}-\mathrm{NC}$ & $2,036,053$ & $2,037,460$ & $2,039,030$ & $2,036,042$ & $2,038,603$ & 2,044 & $2,036,732$ & $2,039,795$ & $2,053,011$ \\
\hline C-C & $2,035,844$ & $2,017,291$ & $2,004,176$ & $2,036,130$ & $2,017,935$ & $2,005,212$ & $2,036,273$ & $2,018,238$ & $2,005,726$ \\
\hline
\end{tabular}


In summary, the results of the sensitivity analysis show that paying past the permissible delay periods while having high demand, and buyers' and vendor's investment rates when the other parameters are low returns the highest system profit. Additional profits generated from investments or delay charges can be used to compensate the losing parties.

\subsection{Managerial Insights}

The following some managerial insights that could be drawn from the results in Sections 8.7 and 8.8:

1. Consignment stock (CS) policy is preferred when the supply chain annual costs of ordering, transaction, storage and in-house financial-holding for upstream players, and in-house financial-holding for downstream players are high. Otherwise, a traditional coordination agreement is preferred. If a supply chain wants to reap other benefits of CS agreements, then it may be necessary that manager works towards reducing the costs that disadvantage CS.

2. CS guarantees the availability of inventory at a downstream facility. In most cases, the upstream player manages the inventory of a downstream player reducing the chances for stock-out situations. It also improves the service level of the downstream player and increases customer satisfaction.

3. CS favors the upstream player since it ships larger quantities in fewer number of transport operations. This policy reduces the costs of transportation and its environmental impact (Bazan et al., 2015).

4. The differences in profits among the four scenarios in the three cases were, in general, minimal. This finding suggests that considering total profit alone may not be a sound managerial decision. Other (cost) factors, not considered in this study, are to be considered when refining decisions. Space is an important issue for many manufacturers. Moving more items to the buyers frees space for other products and parts. CS, for example, becomes favored by a manufacturer who has a limited space and it is expensive to expand its storage capacity. However, it may not favor CS with its suppliers as doing so may eat up its limited space capacity.

5. Allowing downstream players to delay their payments past their due dates may entice these players to search for investments with higher rates of return than the interest that an 
upstream player charges on outstanding balances. Buyers in non-competing geographic locations may pool their resources to increase their investment collateral capacity. Having a financial institution managing payment transactions, financing of the supply chain operations and investments of the players may not be a bad idea as it can pool supply chain resources and diversify its investment opportunities.

\subsection{Conclusion}

In this chapter, a three-level supply chain system consisting of multiple suppliers, a vendor, and multiple buyers has been developed by considering four coordination scenarios. The first scenario adopts traditional coordination policies between adjacent players (suppliers-vendor and vendorbuyers) in the supply chain. The fourth scenario adopts consignment agreements between adjacent players. The second and third scenarios are combinations of traditional and consignment coordination policies. This chapter considered equal-sized payments at equal time intervals are made to settle outstanding balances when a consignment agreement is adopted, and three cases of delay-in-payments between upstream and downstream players. Numerical examples were provided and a sensitivity analysis was performed to illustrate the behavior of the developed scenarios/models and to investigate the effects of different parameters in choosing the scenario that maximizes supply chain profitability. Some managerial insights were provided to help managers or decision makers in choosing the best scenario that fits their business.

The numerical results showed that a combined coordination policy returns the highest system profit. They also showed that offering a delay-in-payment generates an additional profit for the system. Moreover, it has been shown that optimizing the number of payments reduces costs and increases the system profit. The results further showed that the scenario that maximizes the system profit could be different from the one that some players favor. Since this chapter considered a centralized decision-making process, it is, therefore, implicitly assumed that a player who loses from the centralization is compensated from the pool of profits that it generates. The differences in profits among the four scenarios for all cases were found to be very small. This finding may caution managers not to rely on profit as a performance measure, but to consider other tangible and intangible factors. 
The results of the sensitivity analysis showed that choosing the best scenario depends on the values of the input parameters. They also showed that an increase in the demand rates of the buyers and in the buyers' and vendor's interest rates increase supply chain profitability. On the other hand, an increase in the buyers' storage-holding and ordering costs and the vendor's raw-materials storageholding and setup costs reduce the profit of the system. Delay-in-payments was also shown to increase supply chain profitability.

The results also showed that increasing the length of the delay period is better for the system than reducing it especially when interest is charged. Although offering delay-in-payments increases the profit, it negatively affects the upstream players. Therefore, increasing the charged interest rate and/or reducing the interest-free period reduce the losses for some players; this could be coupled with some sort of profit sharing contract. Moreover, if the difference in profits between two scenarios is small, it is better to choose the one that serves most of the players. In addition, although adopting consignment stock agreements by the players did not return the highest profits, in some situations, it may have the potential benefit of increasing the service level among the players by eliminating out-of-stock situations. Businesses that have noticeable fluctuations in demand are better off with a consignment stock agreement as downstream players are served better this way.

This chapter can be extended by considering stochastic demand that depends on selling prices, delivery lead-time and product differentiation with or without considering product customization (Batarfi et al., 2016). A more complex multi-tier supply chain structure could also be considered (Jaber and Goyal, 2009). Unlike this chapter, the buyers or suppliers may not all agree to the same agreements with the vendor. That is, some buyers may agree to a consignment policy, while others may prefer a traditional coordination policy. Future work may consider accounting for warehouse capacity, service-level, and/or transportation. Some or a combination of the above extensions would be steps forward towards the advancement of the theory and practice of inventory and supply chain management. 


\section{Chapter 9: Conclusions and Recommendations}

Supply chain management (SCM) has been the center of interest for businesses as it provides the tools to increase their performance, mainly, profitability and customer satisfaction. Many SCM researchers have investigated different coordination mechanisms to determine which one is best for which situation. Some of them developed models that focused on the internal chain of a business to enhance the flow of materials, financial transactions, and information between different departments. Other researchers, focused on the external supply chain to enhance similar flows between different companies working together to deliver final products to customers. The second group developed different inventory models, some of which took a decentralized approach by focusing on improving a system of a single player without considering the ripple effect that such an approach may have on the other party in the chain, while others took a centralized approach by considering a supply chain as one system. This thesis adopts the second approach, which is commonly referred to in the literature as joint-economic-lot-size (JELS) problem.

Although the consignment stock (CS), a form of JELS, has been practiced for a long time by businesses, it is a relatively recent topic for the academic community. Some of the published studies are based on assumptions limit their applicability. The available studies in the literature have ignored some important aspects published in actual CS contracts. Also, none of the available models in the literature investigated CS in more than two-level supply chain systems including payment schemes, payment delays, and multiple players. This thesis addressed these limitations in the literature and developed models that are to some extent representation of real CS business contracts. These models were also modified to adopt a traditional coordination policy. A comparison between the CS and traditional coordination models was performed to draw insights into their performance and limitations. Following are the four main contributions of this thesis to the consignment stock and supply chain literature.

In Chapter 5, a two-level supply chain model was developed to investigate where the vendor and the buyer agreed to a consignment stock policy the effects of different payment schemes between the vendor and the buyer on the inventory policy and the profitability of the system. The model addressed a limitation in the CS literature as all studies assumed that payments are made to the vendor by the buyer on a continuous basis. The model showed that adopting the scheme in which the buyer makes equal-sized payments at equal intervals is profitable and practical. The results 
showed that optimizing the number of payments in a buyer's cycle increases profitability. For example, reducing the number of payments and pushing them to the end of the cycle provides the buyer with more time to invest its money and accruing interest on sales before paying the vendor. The loss incurred by the vendor could be compensated by adopting a profit-sharing mechanism, which shows to have a positive impact on the system and results in a win-win situation for collaborating parties. Finally, the results of this model showed that different values of the input parameters affect the choice of which payment scheme is best for the system.

In Chapter 6, the model of Chapter 5 was extended to account for a delay-in-payment. Payment methods and payment schedules are two observed realities of CS contracts. Three different scenarios of delay-in-payments were developed and investigated. The results showed that offering the buyer a permissible period to settle its account is more beneficial for the system than paying the vendor at the time of the invoice. The buyer was assumed to invest its sales revenue for the length of the delay period and earn interest, which increases its total revenue and that of the chain. The results also showed that the profitability of the system increases when the buyer pays the vendor after the set due date. The vendor charges interest on payments passed their due dates. The system was shown to have the better profitability when the interest charged by the vendor is less than the interest earned by the buyer. The results suggested that having a profit sharing agreement between the vendor and the buyer may be necessary for some situations. The results of this chapter also showed that the CS policy is more flexible than the traditional policy and returns higher system's profit.

In Chapter 7, a three-level (supplier-vendor-buyer) supply chain model was developed and investigated for four coordination scenarios (combination of traditional and CS). The model assumed a scheme of equal-sized payments at equal intervals between the vendor and the buyer and different storage-holding costs. The main finding of this model is that a combination of traditional and CS policies returned highest system's profit. Similar to previous models, the results also showed that incorporating a profit sharing or a price discount mechanism helps in compensating the losing player from implementing the agreement. The values of the input parameters were shown to have a significant on the performance of the model. Although the combination of the two policies returned the highest profit for the system, the results showed that 
adopting a CS agreement by all players comes in the second place (with slightly lower profit) in most cases this providing managers with some flexibility.

In Chapter 8, the model developed in Chapter 7 was extended to assume multiple suppliers and buyers. The fourth model of this thesis considered a scheme of equal-sized payments at equal time intervals to settle owing balances between the vendor and its suppliers, and the buyers and the vendor. The fourth model also considered three cases of delay-in-payments. Similar to the third model, the results showed that a combined coordination policy returns the highest system profit. They also showed the number of payments reduces costs and increases profits. The results showed that the scenario that maximizes the system's profit could be different from the one that some players favor. Since this thesis considered a centralized decision-making process, it is, therefore, implicitly assumed that a player who loses from the centralization is compensated from the pool of profits that it generates. The results further showed that adopting delay-in-payments increases the system's profit. It was also shown that longer periods of delay in payments profitable for the supply chain especially when the interest charged by an upstream player is lower than the interest earned by a downstream one. In addition, increasing the charged interest rate and/or reducing the interest-free period reduce losses. Although it was found that adopting consignment stock agreements by the players did not return the highest result, in some situations, it may have the potential benefit of increasing the service level among the players by eliminating the out of stock situations. Businesses that have noticeable fluctuations in demand are better off with a consignment stock agreement as downstream players are served better this way.

The performance of the developed models depends on the accuracy of the values of the input parameters. Operations managers should be careful when setting the values of the input parameters for the developed models as they significantly affect the supply chain profitability.

In general, CS has a lot of benefits for the upstream and the downstream players and for the system if managed properly. The upstream player benefits from CS when it has limited storage space, it is cheaper to store items at the facility of a downstream player, products are slow moving or newly launched, and the market is competitive.

CS helps the downstream player in generating more profit by investing its sales revenue before making the payment and enhancing product availability. It reduces lost sales, increases customer 
satisfaction, eliminates the costs of removing or replacing expired, unsold or unwanted items, and reduces ordering and transaction costs.

For the system, CS has many benefits such as ensuring better management and services, enhancing collaboration between players, ensuring product availability, reducing bullwhip effect, and increasing profitability.

One could extend the models developed in this thesis in several directions. For example, accounting for transportation costs and considering lead-times for delivery of shipments and the transfer of payments with stochastic demand. Assuming that the vendor's production and inspection process are imperfect would also be an interesting extension. Choosing the suppliers based on the quality of the product could be a nice topic. Looking at different payment schemes or payment methods (i.e. in cash or credit) or using a net present value approach to modeling the problem. Studying the effect or the impact of supply reliability for a system that produces perishable items on these methods or schemes would be a great idea. The length of a delay-inpayment is a decision variable rather than dictated. Other future extensions include multi-tier (Jaber and Goyal, 2009), multi-products and reverse logistics (Salema et al., 2007), warehouse capacity (Lee and Wang, 2008), environmental issues (Bazan et al., 2015; 2016). One can use the models of this thesis to develop a decision tool that benefits managers in operations and purchasing departments. For example, software that tells managers when to invoice buyers, whether or not to offer delay-in-payments, calculates the length of the delay period and the balances owed by a downstream player. The software could also factor in the loyalty of some customers into offering trade credits. The decision support tool should have a database of the purchasing and ordering data of the firm and customers. 


\section{Appendices}

\section{Appendix 1}

Table 10-1. Closes as stated in the consignment stock contract for companies

\begin{tabular}{|c|c|}
\hline Company name & Close as stated in the contract \\
\hline LBMA & $\begin{array}{l}\text { "The Consignor reserves the right to charge interest on any amount remaining unpaid } \\
\text { after the due date for payment, from the due date to the date of payment, at the rate of } \\
{[[\mathrm{X}] \% \text { over the } 1 \text { month LIBOR rate of the British Bankers' Association in force }} \\
\text { from time to time]." }\end{array}$ \\
\hline Trelleborg & $\begin{array}{l}\text { "Each Invoice for Products shall be for those Products purchased in the preceding } \\
\text { Month as advised by the Customer under clause } 5 \text { (subject to a Joint inspection and } \\
\text { stock count in accordance with clause } 4 \text { ). Payment will be due } 30 \text { days following the } \\
\text { date of invoice." }\end{array}$ \\
\hline $\begin{array}{l}\text { CARFAC } \\
\text { Saskatchewan } \\
\text { Visual Artists }\end{array}$ & $\begin{array}{l}\text { "The Dealer is responsible for billing and collecting all amounts owed by a purchaser } \\
\text { for Consigned Works. Within fourteen (14) days of receipt of any payment pursuant } \\
\text { to a Sale, the Dealer shall pay to the Artist the total sale price less any commissions } \\
\text { owed to the Dealer under this Agreement (the "Artist Payment"). Interest on overdue } \\
\text { Artist Payments shall accrue and be owed to the Artist at the rate of twelve percent } \\
(12 \%) \text { per year, compounded monthly, until actual payment to the Artist." }\end{array}$ \\
\hline $\begin{array}{l}\text { The Newcastle } \\
\text { upon Tyne } \\
\text { Hospitals NHS } \\
\text { Foundation }\end{array}$ & $\begin{array}{l}\text { "Within } 14 \text { days of the use by the Customer of a Consignment Product, the Customer } \\
\text { shall raise a purchase order to replenish the Consignment Stock and the Company } \\
\text { shall raise and issue to the Customer an invoice for the sale of such Consignment } \\
\text { Product to the Customer." }\end{array}$ \\
\hline Trust & $\begin{array}{l}\text { "The Customer shall make payment in full for each purchase of Consignment } \\
\text { Products to replenish the Consignment Stock within } 30 \text { days from the receipt of an } \\
\text { invoice issued by Company. Payment shall be made by BACS to the Company." }\end{array}$ \\
\hline $\begin{array}{l}\text { Stone Art } \\
\text { Manufacturing }\end{array}$ & $\begin{array}{l}\text { "STONE ART MANUFACTURING shall on a weekly basis perform stock counts. } \\
\text { These stock counts will reflect unit sales. The units sold shall be invoiced to the } \\
\text { Distributor. Payment of these unit sales shall be paid to STONE ART } \\
\text { MANUFACTURING with } 7 \text { days of invoiced date." }\end{array}$ \\
\hline $\begin{array}{l}\text { NASCO } \\
\text { Distributor } \\
\text { Sales }\end{array}$ & $\begin{array}{l}\text { "Payment to consignee will occur N-45 terms after the close of the transactions for } \\
\text { any month. This is to allow for possible returns from customer(s). As an example, if } \\
\text { Nasco sells a consigned item on February } 15 \text { th, or on any day in February, payment } \\
\text { of the _ percent will be due and payable on April 15th, which is } 45 \text { days after the } \\
\text { close of February." }\end{array}$ \\
\hline
\end{tabular}




\section{Appendix 2}

A comparison between the calculation of the financial-holding (losing opportunity) cost in Chapter 5 and Scenario 1, 2, and 3 in Chapter 6 is made to show the difference between them.

In Chapter 5, the financial-holding cost is equal to

$$
C_{v}^{o p}=c_{b} I_{v}\left(n q-(n-1) \frac{D q}{2 P}\right)
$$

In Chapter 6, the financial-holding cost for Scenario 1, 2, and 3 is equal to

$$
C_{v, 1}^{h, f}=c_{b} I_{v}\left(\left(\frac{m_{1}+1}{2 m_{1}}\right) n_{1} q_{1}-\left(n_{1}-1\right) \frac{D q_{1}}{2 P}\right)
$$

Assume that the values of $c_{b}, I_{v}, n, q, D$, and $P$, are fixed numbers in both equations 10-1 and 10-2. Therefore, Eq. (10-1) = Eq. (10-2) if and only if $m_{1}=1$ in Eq. (10-2). If $m_{1}>1$, $\left(m_{1}+1 / 2 m_{1}\right) n_{1} q_{1}$ part is the only difference between the two equations (10-1) and (10-2) since $c_{b} I_{v}$ and $(n-1) D q / 2 P$ are similar in both equations. This means that if $\left(m_{1}+1 / 2 m_{1}\right)$ in Eq. (10-2) returns a value less than one, Eq. (10-2) returns a lower cost than Eq. (10-1) since its $\left(m_{1}+1 / 2 m_{1}\right)$ is equal to one. To understand the behavior of Eq. (10-2), the limit for $\left(m_{1}+1 / 2 m_{1}\right)$ is taken when $m_{1} \rightarrow \infty$.

$\lim _{m_{1} \rightarrow \infty}\left(\frac{m_{1}+1}{2 m_{1}}\right)=\lim _{m_{1} \rightarrow \infty}\left(\frac{m_{1}}{2 m_{1}}+\frac{1}{2 m_{1}}\right)=\lim _{m_{1} \rightarrow \infty}\left(\frac{1}{2}+\frac{1}{2 m_{1}}\right)=\frac{1}{2}+0=\frac{1}{2}$

Taking the limit shows that Eq. (10-2) returns lower total cost as $m_{1}$ goes to infinity.

The above also applies on the two financial-holding cost for Scenario 2 and 3 while assuming that $\alpha$ and $\beta$ are also fixed numbers. The financial-holding for the second and the third scenario is

$$
\begin{aligned}
& C_{v, 2}^{h, f}=c_{b} I_{v}\left(\left(\frac{m_{2}+1+2 \alpha}{2 m_{2}}\right) n_{2} q_{2}-\left(n_{2}-1\right) \frac{q_{2} D}{2 P}\right) \\
& C_{v, 3}^{h, f}=c_{b} I_{v}\left(\left(\frac{m_{3}+1+2(\alpha+\beta(1+\alpha))}{2 m_{3}}\right) n_{3} q_{3}-\left(n_{3}-1\right) \frac{q_{3} D}{2 P}\right)
\end{aligned}
$$

By taking the limit to $\left(\frac{m_{2}+1+2 \alpha}{2 m_{2}}\right)$ when $m_{2}$ goes to infinity, and $\left(\frac{m_{3}+1+2(\alpha+\beta(1+\alpha))}{2 m_{3}}\right)$ when $m_{3}$ goes to infinity, the results are 


$$
\begin{gathered}
\lim _{m_{2} \rightarrow \infty}\left(\frac{m_{2}+1+2 \alpha}{2 m_{2}}\right)=\lim _{m_{2} \rightarrow \infty}\left(\frac{m_{2}}{2 m_{2}}+\frac{1}{2 m_{2}}+\frac{2 \alpha}{2 m_{2}}\right)=\lim _{m_{2} \rightarrow \infty}\left(\frac{1}{2}+\frac{1}{2 m_{2}}+\frac{2 \alpha}{2 m_{2}}\right)=\frac{1}{2}+0+0 \\
=\frac{1}{2}
\end{gathered}
$$

$$
\begin{gathered}
\lim _{m_{3} \rightarrow \infty}\left(\frac{m_{3}+1+2(\alpha+\beta(1+\alpha))}{2 m_{3}}\right)=\lim _{m_{3} \rightarrow \infty}\left(\frac{m_{3}}{2 m_{3}}+\frac{1}{2 m_{3}}+\frac{2(\alpha+\beta(1+\alpha))}{2 m_{3}}\right) \\
=\lim _{m_{3} \rightarrow \infty}\left(\frac{1}{2}+\frac{1}{2 m_{3}}+\frac{2(\alpha+\beta(1+\alpha))}{2 m_{3}}\right)=\frac{1}{2}+0+0=\frac{1}{2}
\end{gathered}
$$

This shows that Eqs. (10-3) and (10-4) return lower total cost than Eq. (10-1) as $m_{2}$ and $m_{3}$, respectively, go to infinity.

\section{Appendix 3}

The denominator of Eq. (6-6) is:

$$
n_{1}^{2}\left[\left(h_{v, f}+h_{b, s}\right)\left(1-\frac{D}{P}\right)+\left(h_{v, f}-c_{c} I_{b}\right) \frac{1}{m_{1}}+\left(h_{v, s}+h_{v, f}+h_{b, s}\right) \frac{D}{n_{1} P}\right]
$$

Since the values of $h_{v, f}, h_{b, s}, h_{v, s}, n_{1}, m_{1}, c_{c} I_{b}, D$, and $P>0$, where $0<\frac{D}{P}<1$, then, we have

$$
\left(h_{v, f}+h_{b, s}\right)\left(1-\frac{D}{P}\right)>0, \text { and }\left(h_{v, s}+h_{v, f}+h_{b, s}\right) \frac{D}{n_{1} P}>0 .
$$

Now, we need to show that $\left(h_{v, f}-c_{c} I_{b}\right) \frac{1}{m_{1}}<\left(h_{v, f}+h_{b, s}\right)\left(1-\frac{D}{P}\right)+\left(h_{v, s}+h_{v, f}+h_{b, s}\right) \frac{D}{n_{1} P}$.

The minimum value that $n_{1}$ and $m_{1}$ can take is 1, i.e. $n_{1}=m_{1}=1$. So,

$$
\begin{aligned}
& \left(h_{v, f}-c_{c} I_{b}\right)<\left(h_{v, f}+h_{b, s}\right)\left(1-\frac{D}{P}\right)+\left(h_{v, s}+h_{v, f}+h_{b, s}\right) \frac{D}{P} \\
& h_{v, f}-c_{c} I_{b}<h_{v, f}+h_{b, s}-\left(h_{v, f}+h_{b, s}\right) \frac{D}{P}+\left(h_{v, s}+h_{v, f}+h_{b, s}\right) \frac{D}{P} \\
& -c_{c} I_{b}<h_{b, s}-\left(h_{v, f}+h_{b, s}\right) \frac{D}{P}+\left(h_{v, s}+h_{v, f}+h_{b, s}\right) \frac{D}{P} \\
& -c_{c} I_{b}<h_{b, s}-\left(h_{v, f}+h_{b, s}\right) \frac{D}{P}+h_{v, s} \frac{D}{P}+\left(h_{v, f}+h_{b, s}\right) \frac{D}{P}
\end{aligned}
$$


$-c_{c} I_{b}<h_{b, s}+h_{v, s} \frac{D}{P}$

$c_{c} I_{b}+h_{b, s}+h_{v, s} \frac{D}{P}>0$

, which is correct. So the denominator is positive when $n_{1}=m_{1}=1$.

When $n_{1}$ and $m_{1}$ approach large values, then $\left(h_{v, f}-c_{c} I_{b}\right) \frac{1}{m_{1}}<\left(h_{v, f}+h_{b, s}\right)\left(1-\frac{D}{P}\right)+$ $\left(h_{v, s}+h_{v, f}+h_{b, s}\right) \frac{D}{n_{1} P}$ becomes $\left(h_{v, f}+h_{b, s}\right)\left(1-\frac{D}{P}\right)>0$, which is also true.

From the above, we deduce that the denominator for Eq. (6-6) is always positive.

The denominators of Eqs. (6-11) and (6-18) are also positive and can be shown so in an identical approach to the one presented in this appendix.

\section{Appendix 4}

According to what has been shown in Grubbström (1996), and more generally in Grubbström and Erdem (1999) and in Zanoni and Grubbstrom (2004) the minimization of a function $f(x)$ where $x$ is a decision variable, can be expressed as

$$
f(x)=a x+\frac{b}{x}+c=a x(x-\sqrt{b / a})^{2}+2 \sqrt{a b}+c
$$

The coefficients ( $a$ and $b$ ) in the function $f(x)$ above are positive. The quadratic term $(x-\sqrt{b / a})^{2}$ is positive and the optimum solution that minimizes $f(x)$ is found when $x^{*}=x=$ $\sqrt{b / a}$. Then, the minimum value of $f(x)$ is calculated such as $f\left(x^{*}\right)=2 \sqrt{a b}+c$, where $a, b$ and $c$ are positive coefficients.

Since the models developed in Chapter 6 are maximization functions and the revenue of the system and some costs' factors are constants, the maximum profit can be achieved by minimizing the total variable cost of the system $\left(T V C_{s, i}\right)$, where $i=1,2$, and 3. Using the above approach, 
$\max \left[T P_{s, i}\left(q_{i}^{*}\left(n_{i}^{*}, m_{i}^{*}\right), n_{i}^{*}, m_{i}^{*}\right)\right]=$ constant revenue and costs -

$\min \left[T V C_{s, i}\left(q_{i}^{*}\left(n_{i}^{*}, m_{i}^{*}\right), n_{i}^{*}, m_{i}^{*}\right)\right]=\left(c_{c}-\gamma c_{p}-c_{p r}\right) D-f\left(x^{*}\right)=\left(c_{c}-\gamma c_{p}-c_{p r}\right) D-$

$2 \sqrt{a b}+c$.

This approach can be straightly applied on Scenario 1, where the total profit for the system (Eq. 6-5) is equal to

$$
\begin{aligned}
& T P_{s, 1}\left(n_{1}, m_{1}, q_{1}\right)=\left(c_{c}-\gamma c_{p}-c_{p r}\right) D-\left[\left(S_{v}+n_{1} O_{b}+m_{1} c_{t}\right) \frac{D}{n_{1} q_{1}}+\right. \\
& \left.\frac{\left(h_{v, f}+h_{b, s}\right) n_{1} q_{1}}{2}\left(1-\frac{D}{P}\right)+\left(h_{v, f}-c_{c} I_{b}\right) \frac{n_{1} q_{1}}{2 m_{1}}+\left(h_{v, s}+h_{v, f}+h_{b, s}\right) \frac{q_{1} D}{2 P}\right]
\end{aligned}
$$

, which is maximized by choosing the optimal value of $n_{1}, m_{1}, q_{1}$, where the optimal size of the shipments can be calculated using Eq. (6-6) while searching for the values of $n_{1}$ and $m_{1}$ (i.e. Jaber and Goyal, 2008). The optimum size of shipments that maximizes Eq. (6-5) is

$$
q_{1}\left(n_{1}, m_{1}\right)=\sqrt{\frac{\left(s_{v}+n_{1} o_{b}+m_{1} c_{t}\right) \frac{D}{n_{1}}}{\frac{\left(h_{v, f}+h_{b, s}\right) n_{1}}{2}\left(1-\frac{D}{P}\right)+\left(h_{v, f}-c_{c} I_{b}\right) \frac{n_{1}}{2 m_{1}}+\left(h_{v, s}+h_{v, f}+h_{b, s}\right) \frac{D}{2 P}}}
$$

By substituting Eq. 6-6 in 6-5, the total profit of the system is expressed as

$$
\begin{aligned}
& T P_{s, 1}\left(q_{1}^{*}\left(n_{1}, m_{1}\right), n_{1}, m_{1}\right)=\left(c_{c}-\gamma c_{p}-c_{p r}\right) D-2 \times \\
& \sqrt{\left[\frac{\left(h_{v, f}+h_{b, s}\right) n_{1}}{2}\left(1-\frac{D}{P}\right)+\left(h_{v, f}-c_{c} I_{b}\right) \frac{n_{1}}{2 m_{1}}+\left(h_{v, s}+h_{v, f}+h_{b, s}\right) \frac{D}{2 P}\right]\left[\left(S_{v}+n_{1} O_{b}+m_{1} c_{t}\right) \frac{D}{n_{1}}\right]}
\end{aligned}
$$

\section{The total variable costs' $\left(T V C_{s, 1}\right)$ joint optimization procedure for $n_{1}, m_{1}$, and $q_{1}$ :}

As seen in Eq. (10-6), the total variable costs for Scenario 1 is equal to 


$$
\begin{aligned}
& T V C_{s, 1}\left(q_{1}^{*}\left(n_{1}, m_{1}\right), n_{1}, m_{1}\right)=2 \times \\
& \sqrt{\left[\begin{array}{c}
\frac{\left(h_{v, f}+h_{b, s}\right)\left(S_{v}+n_{1} o_{b}+m_{1} c_{t}\right) D}{2}\left(1-\frac{D}{P}\right)+\frac{\left(h_{v, f}-c_{c} I_{b}\right)\left(s_{v}+n_{1} o_{b}+m_{1} c_{t}\right) D}{2 m_{1}} \\
+\frac{\left(h_{v, s}+h_{v, f}+h_{b, s}\right)\left(s_{v}+n_{1} o_{b}+m_{1} c_{t}\right) D^{2}}{2 n_{1} P}
\end{array}\right]}
\end{aligned}
$$

To show that the terms under the square root in Eq. (10-7) are positive, set $n_{1}=1$ and $m_{1}=1$, then the equation is written as

$$
\begin{aligned}
& {\left[T V C_{s, 1}\left(q_{1}^{*}(1,1), 1,1\right)\right]^{2}=2\left[\left(h_{v, f}+h_{b, s}\right)\left(S_{v}+O_{b}+c_{t}\right) D\left(1-\frac{D}{P}\right)+\left(h_{v, f}-\right.\right.} \\
& \left.\left.c_{c} I_{b}\right)\left(S_{v}+O_{b}+c_{t}\right) D+\frac{\left(h_{v, s}+h_{v, f}+h_{b, s}\right)\left(S_{v}+O_{b}+c_{t}\right) D^{2}}{P}\right]
\end{aligned}
$$

with $\left(h_{v, f}+h_{b, s}\right)\left(1-\frac{D}{P}\right)+\left(h_{v, f}-c_{c} I_{b}\right)+\left(h_{v, f}+h_{b, s}+h_{v, S}\right) \frac{D}{P}>0$

$2 h_{v, f}+h_{b, s}-c_{c} I_{b}+h_{v, s} \frac{D}{P}>0$

$2 h_{v, f}+h_{b, s}+h_{v, s} \frac{D}{P}>c_{c} I_{b}$, which will be.

\section{To find the close form of $n_{1}^{*}$ :}

For $n_{1}>1$ and $m_{1}=1$, Eq. (10-7) is written as

$$
\begin{aligned}
& {\left[\operatorname{TVC}_{s, 1}\left(q_{1}^{*}\left(n_{1}, 1\right), n_{1}, 1\right)\right]^{2} } \\
&=2\left[\left(h_{v, f}+h_{b, s}\right)\left(1-\frac{D}{P}\right) O_{b} D n_{1}+\left(h_{v, f}-c_{c} I_{b}\right) O_{b} D n_{1}\right. \\
&+ \frac{\left(h_{v, s}+h_{v, f}+h_{b, s}\right)\left(S_{v}+c_{t}\right) D^{2}}{n_{1} P}+\left(h_{v, f}+h_{b, s}\right)\left(S_{v}+c_{t}\right) D\left(1-\frac{D}{P}\right) \\
&\left.+\left(h_{v, f}-c_{c} I_{b}\right)\left(S_{v}+c_{t}\right) D+\frac{\left(h_{v, s}+h_{v, f}+h_{b, s}\right) O_{b} D^{2}}{P}\right]
\end{aligned}
$$

, which is the form of $\left[\operatorname{TV} C_{s, 1}\left(q_{1}^{*}\left(n_{1}, 1\right), n_{1}, 1\right)\right]^{2}=a n_{1}+\frac{b}{n_{1}}+c$, therefore,

$$
a=2 O_{b} D\left[\left(h_{v, f}+h_{b, s}\right)\left(1-\frac{D}{P}\right)+h_{v, f}-c_{c} I_{b}\right]
$$




$$
\begin{gathered}
b=\frac{2\left(h_{v, s}+h_{v, f}+h_{b, s}\right)\left(S_{v}+c_{t}\right) D^{2}}{P} \\
c=2\left(S_{v}+c_{t}\right) D\left[\left(h_{v, f}+h_{b, s}\right)\left(1-\frac{D}{P}\right)+h_{v, f}-c_{c} I_{b}\right] \\
+\frac{2 O_{b} D^{2}\left(h_{v, s}+h_{v, f}+h_{b, s}\right)}{P}
\end{gathered}
$$

with

$$
n_{1}^{*}=\sqrt{b / a}=\sqrt{\frac{\left(h_{v, s}+h_{v, f}+h_{b, s}\right)\left(s_{v}+c_{t}\right) D}{o_{b} P\left[\left(h_{v, f}+h_{b, s}\right)\left(1-\frac{D}{P}\right)+h_{v, f}-c_{c} I_{b}\right]}}
$$

, where $\left(h_{v, f}+h_{b, s}\right)\left(1-\frac{D}{P}\right)+h_{v, f}>c_{c} I_{b}$.

\section{To find the close form of $m_{1}^{*}$ :}

For $n_{1}=1$ and $m_{1}>1$, Eq. (10-7) is written as

$$
\begin{aligned}
{\left[\operatorname{TVC}_{s, 1}\left(q_{1}^{*}\left(1, m_{1}\right), 1, m_{1}\right)\right]^{2} } & \\
= & 2\left[\left(h_{v, f}+h_{b, s}\right) m_{1} c_{t} D\left(1-\frac{D}{P}\right)+\frac{\left(h_{v, s}+h_{v, f}+h_{b, s}\right) m_{1} c_{t} D^{2}}{P}\right. \\
& +\frac{\left(h_{v, f}-c_{c} I_{b}\right)\left(S_{v}+O_{b}\right) D}{m_{1}}+\left(h_{v, f}+h_{b, s}\right)\left(S_{v}+O_{b}\right) D\left(1-\frac{D}{P}\right) \\
& \left.+\left(h_{v, f}-c_{c} I_{b}\right) c_{t} D+\frac{\left(h_{v, s}+h_{v, f}+h_{b, s}\right)\left(S_{v}+O_{b}\right) D^{2}}{P}\right]
\end{aligned}
$$

, which is the form of $\left[T P_{s, 1}\left(q_{1}^{*}\left(1, m_{1}\right), 1, m_{1}\right)\right]^{2}=a m_{1}+\frac{b}{m_{1}}+c$, therefore,

$$
\begin{gathered}
a=2 c_{t} D\left[\left(h_{v, f}+h_{b, s}\right)\left(1-\frac{D}{P}\right)+\frac{\left(h_{v, s}+h_{v, f}+h_{b, s}\right) D}{P}\right] \\
b=2\left[\left(h_{v, f}-c_{c} I_{b}\right)\left(S_{v}+O_{b}\right) D\right], \text { where } h_{v, f}>c_{c} I_{b} \\
c=2\left(S_{v}+O_{b}\right) D\left[\left(h_{v, f}+h_{b, s}\right)\left(1-\frac{D}{P}\right)+\frac{\left(h_{v, s}+h_{v, f}+h_{b, s}\right) D}{P}\right] \\
+2\left(h_{v, f}-c_{c} I_{b}\right) c_{t} D
\end{gathered}
$$


with

$$
m_{1}^{*}=\sqrt{b / a}=\sqrt{\frac{\left[\left(h_{v, f}-c_{c} I_{b}\right)\left(S_{v}+O_{b}\right) D\right]}{c_{t} D\left[\left(h_{v, f}+h_{b, s}\right)\left(1-\frac{D}{P}\right)+\frac{\left(h_{v, s}+h_{v, f}+h_{b, s}\right) D}{P}\right]}}
$$

Note that $m_{1}^{*}>1$ if and only if $h_{v, f}>c_{c} I_{b}$, otherwise, $m_{1}^{*}=1$ payment at the end of the cycle, which is the minimum number of payments the buyer makes to the vendor.

By substituting the values of $n_{1}^{*}$ and $m_{1}^{*}$ in Eqs. (10-7) and (6-6), we get the minimum $T V C_{s, 1}$ that maximizes the total profit of the system and $q_{1}^{*}\left(n_{1}^{*}, m_{1}^{*}\right)$, respectively.

Since Scenario 2 and 3 are similar to Scenario 1, the steps above can be applied to find the optimum number of shipments and payments and the size of each shipment. Therefore, for Scenario 2, when $n_{2}=1$ and $m_{2}=1$, then

$$
\begin{aligned}
& {\left[T V C_{s, 2}\left(q_{2}^{*}(1,1), 1,1\right)\right]^{2}=2 \times\left[\left(h_{v, f}+h_{b, s}\right)\left(S_{v}+O_{b}+c_{t}\right) D\left(1-\frac{D}{P}\right)+(2 \alpha+\right.} \\
& \text { 1) } \left.\left(h_{v, f}-c_{c} I_{b}\right)\left(S_{v}+O_{b}+c_{t}\right) D+\frac{\left(h_{v, s}+h_{v, f}+h_{b, s}\right)\left(S_{v}+O_{b}+c_{t}\right) D^{2}}{P}\right]
\end{aligned}
$$

with $\left(h_{v, f}+h_{b, s}\right)\left(1-\frac{D}{P}\right)+(2 \alpha+1)\left(h_{v, f}-c_{c} I_{b}\right)+\left(h_{v, f}+h_{b, s}+h_{v, s}\right) \frac{D}{P}>0$

, therefore, $\frac{2(\alpha+1) h_{v, f}+h_{b, s}+h_{v, s} \frac{D}{P}}{2 \alpha+1}>c_{c} I_{b}$

\section{To find the close form of $n_{2}^{*}$ :}

$$
T V C_{s, 2}\left(q_{2}^{*}\left(n_{2}, m_{2}\right), n_{2}, m_{2}\right)=2 \times
$$

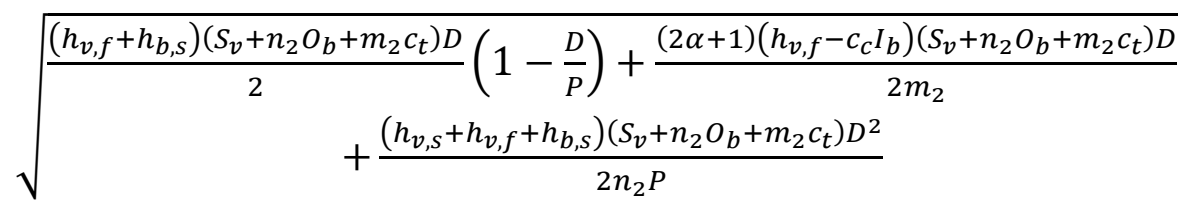

Applying the same steps for $n_{1}^{*}$, when $n_{2}>1$ and $m_{2}=1$, the close form of $n_{2}^{*}$ is equal to 


$$
n_{2}^{*}=\sqrt{b / a}=\sqrt{\frac{\left(h_{v, s}+h_{v, f}+h_{b, s}\right)\left(S_{v}+c_{t}\right) D}{O_{b} P\left[\left(h_{v, f}+h_{b, s}\right)\left(1-\frac{D}{P}\right)+(2 \alpha+1)\left(h_{v, f}-c_{c} I_{b}\right)\right]}}
$$

, where $\frac{\left(h_{v, f}+h_{b, s}\right)\left(1-\frac{D}{P}\right)+(2 \alpha+1) h_{v, f}}{(2 \alpha+1)}>c_{c} I_{b}$.

\section{To find the close form of $m_{2}^{*}$ :}

After applying the steps above for $m_{1}^{*}$ when $n_{2}=1$ and $m_{2}>1$, the close form is found to be equal to

$$
m_{2}^{*}=\sqrt{b / a}=\sqrt{\frac{(2 \alpha+1)\left(h_{v, f}-c_{c} I_{b}\right)\left(S_{v}+O_{b}\right) D}{c_{t} D\left[\left(h_{v, f}+h_{b, s}\right)\left(1-\frac{D}{P}\right)+\frac{\left(h_{v, s}+h_{v, f}+h_{b, s}\right) D}{P}\right]}}
$$

Note that $m_{2}^{*}>1$ if and only if $h_{v, f}>c_{c} I_{b}$, otherwise, $m_{2}^{*}=1$ payment at the end of the cycle, which is the minimum number of payments that the buyer can make.

By substituting the values of $n_{2}^{*}$ and $m_{2}^{*}$ in Eqs. (10-14) and (6-11), we get the minimum $T V C_{s, 2}$ that maximizes the total profit of the system and $q_{2}^{*}\left(n_{2}^{*}, m_{3}^{*}\right)$, respectively.

For Scenario 3, when $n_{3}=1$ and $m_{3}=1$, then

$$
\begin{aligned}
& {\left[T V C_{s, 3}\left(q_{3}^{*}(1,1), 1,1\right)\right]^{2}=2 \times\left[\left(h_{v, f}+h_{b, s}\right)\left(S_{v}+O_{b}+c_{t}\right) D\left(1-\frac{D}{P}\right)+(2 \alpha+1+10-17\right.} \\
& \left.2 \beta(1+\alpha))\left(h_{v, f}-c_{c} I_{b}\right)\left(S_{v}+O_{b}+c_{t}\right) D+\frac{\left(h_{v, s}+h_{v, f}+h_{b, s}\right)\left(S_{v}+O_{b}+c_{t}\right) D^{2}}{P}\right] \\
& \text { with }\left(h_{v, f}+h_{b, s}\right)\left(1-\frac{D}{P}\right)+(2 \alpha+1+2 \beta(1+\alpha))\left(h_{v, f}-c_{c} I_{b}\right)+\left(h_{v, f}+h_{b, s}+h_{v, s}\right) \frac{D}{P}>0 \\
& \text {, therefore, } \frac{2(\alpha+1+\beta(1+\alpha)) h_{v, f}+h_{b, s}+h_{v, s} \frac{D}{P}}{2 \alpha+1+2 \beta(1+\alpha)}>c_{c} I_{b} .
\end{aligned}
$$




\section{To find the close form of $n_{3}^{*}$ :}

$$
\begin{aligned}
& T V C_{s, 3}\left(q_{3}^{*}\left(n_{3}, m_{3}\right), n_{3}, m_{3}\right)=2 \times \\
& \sqrt{\frac{\left(h_{v, f}+h_{b, s}\right)\left(S_{v}+n_{3} O_{b}+m_{3} c_{t}\right) D}{2}\left(1-\frac{D}{P}\right)+\frac{(2 \alpha+1+2 \beta(1+\alpha))\left(h_{v, f}-c_{c} I_{b}\right)\left(S_{v}+n_{3} O_{b}+m_{3} c_{t}\right) D}{2 m_{3}}} \\
& +\frac{\left(h_{v, s}+h_{v, f}+h_{b, s}\right)\left(s_{v}+n_{3} o_{b}+m_{3} c_{t}\right) D^{2}}{2 n_{3} P}
\end{aligned}
$$

Applying the same steps for $n_{1}^{*}$, when $n_{3}>1$ and $m_{3}=1$, the close form of $n_{3}^{*}$ is equal to

$$
n_{3}^{*}=\sqrt{b / a}=\sqrt{\frac{\left(h_{v, s}+h_{v, f}+h_{b, s}\right)\left(S_{v}+c_{t}\right) D}{O_{b} P\left[\left(h_{v, f}+h_{b, s}\right)\left(1-\frac{D}{P}\right)+(2 \alpha+1+2 \beta(1+\alpha))\left(h_{v, f}-c_{c} I_{b}\right)\right]}}
$$

, where $\frac{\left(h_{v, f}+h_{b, s}\right)\left(1-\frac{D}{P}\right)+(2 \alpha+1+2 \beta(1+\alpha)) h_{v, f}}{2 \alpha+1+2 \beta(1+\alpha)}>c_{c} I_{b}$.

\section{To find the close form of $m_{3}^{*}$ :}

After applying the steps above for $m_{1}^{*}$ when $n_{3}=1$ and $m_{3}>1$, the close form is found to be equal to

$$
m_{3}^{*}=\sqrt{b / a}=\sqrt{\frac{(2 \alpha+1+2 \beta(1+\alpha))\left(h_{v, f}-c_{c} I_{b}\right)\left(S_{v}+O_{b}\right) D}{c_{t} D\left[\left(h_{v, f}+h_{b, s}\right)\left(1-\frac{D}{P}\right)+\frac{\left(h_{v, s}+h_{v, f}+h_{b, s}\right) D}{P}\right]}}
$$

Note that $m_{3}^{*}>1$ if and only if $h_{v, f}>c_{c} I_{b}$, otherwise, $m_{3}^{*}=1$ payment at the end of the cycle, which is the minimum number of payments made to the vendor.

By substituting the values of $n_{3}^{*}$ and $m_{3}^{*}$ in Eqs. (10-18) and (6-18), we get the minimum $T V C_{s, 3}$ that maximizes the total profit of the system and $q_{3}^{*}\left(n_{3}^{*}, m_{3 j}^{*}\right)$, respectively.

\section{Appendix 5}

One method that has been used by researchers in literatures extensively to look for a concavity of the profit with respect to its decision variables is to find its second derivative with respect to its 
decision variable, which has to be negative. In this section, a proof of the total profit concavity with respect to its decision variables is given for all scenarios.

\section{C-C Scenario}

\section{a. Proof of concavity with respect to $q_{c}$}

To show that the system's profit for the C-C Scenario is concave in $q_{c}$, we have to show that the second derivative of the system's profit is negative. Therefore, the second derivative for the system's profit is

$\frac{\partial^{2} T P_{C-C}}{\partial q_{c}^{2}}=\frac{\partial^{2}}{\partial q_{c}^{2}}\left(\left(c_{b}^{s}-\gamma \alpha c_{s}^{p}-\alpha c_{s}^{p r}-c_{v}^{p r}\right) D-\left(S_{s}+S_{v}+n_{1} O_{v}+m_{1} c_{v}^{t}+n_{2} O_{b}+\right.\right.$

$\left.m_{2} c_{b}^{t}\right) \frac{D}{n_{2} q_{c}}+\left(\left(n_{1}-1\right) h_{s, f_{2}}-h_{s, s}-h_{s, f_{1}}+\left(n_{1}-1\right) h_{v, s}^{r}\right) \frac{n_{2} \alpha^{2} q_{c} D}{2 n_{1} P_{s}}-\left(n_{2} \alpha h_{v, s}^{r}-\left(n_{2}-\right.\right.$

1) $\left.h_{v, f_{2}}^{f}+h_{v, s}^{f}+h_{v, f_{1}}^{f}-\left(n_{2}-1\right) h_{b, s}\right) \frac{q_{c} D}{2 P_{v}}+\left(c_{b}^{S} I_{b}-h_{v, f_{2}}^{f}\right) \frac{n_{2} q_{c}}{2 m_{2}}-\left(h_{v, f_{2}}^{f}+h_{b, s}\right) \frac{n_{2} q_{c}}{2}-\left(m_{1}+\right.$

1) $\left.h_{s, f_{2}} \frac{n_{2} \alpha q_{c} D}{2 m_{1} P_{v}}\right)$

$\frac{\partial^{2} T P_{C-C}}{\partial q_{c}^{2}}=-\left(S_{S}+S_{v}+n_{1} O_{v}+m_{1} c_{v}^{t}+n_{2} O_{b}+m_{2} c_{b}^{t}\right) \frac{2 D}{n_{2} q_{c}^{3}}<0$

Since the second derivative of the profit function is negative, the total profit is concave in $q_{c}$, which has an optimal solution that maximizes the profit.

\section{b. Proof of concavity with respect to $n_{1}$}

$\frac{\partial^{2} T P_{C-C}}{\partial n_{1}^{2}}=\frac{\partial^{2}}{\partial n_{1}^{2}}\left(\left(c_{b}^{s}-\gamma \alpha c_{s}^{p}-\alpha c_{s}^{p r}-c_{v}^{p r}\right) D-\left(S_{s}+S_{v}+n_{1} O_{v}+m_{1} c_{v}^{t}+n_{2} O_{b}+\right.\right.$

$\left.m_{2} c_{b}^{t}\right) \frac{D}{n_{2} q_{c}}+\left(\left(n_{1}-1\right) h_{s, f_{2}}-h_{s, s}-h_{s, f_{1}}+\left(n_{1}-1\right) h_{v, s}^{r}\right) \frac{n_{2} \alpha^{2} q_{c} D}{2 n_{1} P_{s}}-\left(n_{2} \alpha h_{v, s}^{r}-\left(n_{2}-\right.\right.$

1) $\left.h_{v, f_{2}}^{f}+h_{v, s}^{f}+h_{v, f_{1}}^{f}-\left(n_{2}-1\right) h_{b, s}\right) \frac{q_{c} D}{2 P_{v}}+\left(c_{b}^{S} I_{b}-h_{v, f_{2}}^{f}\right) \frac{n_{2} q_{c}}{2 m_{2}}-\left(h_{v, f_{2}}^{f}+h_{b, s}\right) \frac{n_{2} q_{c}}{2}-\left(m_{1}+\right.$

1) $\left.h_{s, f_{2}} \frac{n_{2} \alpha q_{c} D}{2 m_{1} P_{v}}\right)$

$\frac{\partial^{2} T P_{C-C}}{\partial n_{1}^{2}}=-\left(h_{s, f_{2}}+h_{s, s}+h_{s, f_{1}}+h_{v, s}^{r}\right) \frac{n_{2} \alpha^{2} q_{c} D}{n_{1}^{3} P_{s}}<0$ 
Since the second derivative of the profit function is negative, the total profit is concave in $n_{1}$, which has an optimal solution that maximizes the profit.

\section{c. Proof of concavity with respect to $n_{2}$}

$\frac{\partial^{2} T P_{C-C}}{\partial n_{2}^{2}}=\frac{\partial^{2}}{\partial n_{2}^{2}}\left(\left(c_{b}^{s}-\gamma \alpha c_{s}^{p}-\alpha c_{s}^{p r}-c_{v}^{p r}\right) D-\left(S_{s}+S_{v}+n_{1} O_{v}+m_{1} c_{v}^{t}+n_{2} O_{b}+\right.\right.$

$\left.m_{2} c_{b}^{t}\right) \frac{D}{n_{2} q_{c}}+\left(\left(n_{1}-1\right) h_{s, f_{2}}-h_{s, s}-h_{s, f_{1}}+\left(n_{1}-1\right) h_{v, s}^{r}\right) \frac{n_{2} \alpha^{2} q_{c} D}{2 n_{1} P_{s}}-\left(n_{2} \alpha h_{v, s}^{r}-\left(n_{2}-\right.\right.$

1) $\left.h_{v, f_{2}}^{f}+h_{v, s}^{f}+h_{v, f_{1}}^{f}-\left(n_{2}-1\right) h_{b, s}\right) \frac{q_{c} D}{2 P_{v}}+\left(c_{b}^{S} I_{b}-h_{v, f_{2}}^{f}\right) \frac{n_{2} q_{c}}{2 m_{2}}-\left(h_{v, f_{2}}^{f}+h_{b, s}\right) \frac{n_{2} q_{c}}{2}-\left(m_{1}+\right.$

1) $\left.h_{s, f_{2}} \frac{n_{2} \alpha q_{c} D}{2 m_{1} P_{v}}\right)$

$\frac{\partial^{2} T P_{C-C}}{\partial n_{2}^{2}}=-\left(S_{s}+S_{v}+n_{1} O_{v}+m_{1} c_{v}^{t}+m_{2} c_{b}^{t}\right) \frac{2 D}{n_{2}^{3} q_{c}}<0$

Since the second derivative of the profit function is negative, the total profit is concave in $n_{2}$, which has an optimal solution that maximizes the profit.

\section{d. Proof of concavity with respect to $m_{1}$}

$\frac{\partial^{2} T P_{C-C}}{\partial m_{1}^{2}}=\frac{\partial^{2}}{\partial m_{1}^{2}}\left(\left(c_{b}^{s}-\gamma \alpha c_{s}^{p}-\alpha c_{s}^{p r}-c_{v}^{p r}\right) D-\left(S_{s}+S_{v}+n_{1} O_{v}+m_{1} c_{v}^{t}+n_{2} O_{b}+\right.\right.$

$\left.m_{2} c_{b}^{t}\right) \frac{D}{n_{2} q_{c}}+\left(\left(n_{1}-1\right) h_{s, f_{2}}-h_{s, s}-h_{s, f_{1}}+\left(n_{1}-1\right) h_{v, s}^{r}\right) \frac{n_{2} \alpha^{2} q_{c} D}{2 n_{1} P_{s}}-\left(n_{2} \alpha h_{v, s}^{r}-\left(n_{2}-\right.\right.$

1) $\left.h_{v, f_{2}}^{f}+h_{v, s}^{f}+h_{v, f_{1}}^{f}-\left(n_{2}-1\right) h_{b, s}\right) \frac{q_{c} D}{2 P_{v}}+\left(c_{b}^{s} I_{b}-h_{v, f_{2}}^{f}\right) \frac{n_{2} q_{c}}{2 m_{2}}-\left(h_{v, f_{2}}^{f}+h_{b, s}\right) \frac{n_{2} q_{c}}{2}-\left(m_{1}+\right.$

1) $\left.h_{s, f_{2}} \frac{n_{2} \alpha q_{c} D}{2 m_{1} P_{v}}\right)$

$\frac{\partial^{2} T P_{C-C}}{\partial m_{1}^{2}}=-h_{s, f_{2}} \frac{n_{2} \alpha q_{c} D}{m_{1}^{3} P_{v}}<0$

Since the second derivative of the profit function is negative, the total profit is concave in $m_{1}$, which has an optimal solution that maximizes the profit. 


\section{e. Proof of concavity with respect to $\mathrm{m}_{2}$}

$\frac{\partial^{2} T P_{C-C}}{\partial m_{2}^{2}}=\frac{\partial^{2}}{\partial m_{2}^{2}}\left(\left(c_{b}^{s}-\gamma \alpha c_{s}^{p}-\alpha c_{s}^{p r}-c_{v}^{p r}\right) D-\left(S_{s}+S_{v}+n_{1} O_{v}+m_{1} c_{v}^{t}+n_{2} O_{b}+\right.\right.$

$\left.m_{2} c_{b}^{t}\right) \frac{D}{n_{2} q_{c}}+\left(\left(n_{1}-1\right) h_{s, f_{2}}-h_{s, s}-h_{s, f_{1}}+\left(n_{1}-1\right) h_{v, s}^{r}\right) \frac{n_{2} \alpha^{2} q_{c} D}{2 n_{1} P_{s}}-\left(n_{2} \alpha h_{v, s}^{r}-\left(n_{2}-\right.\right.$

1) $\left.h_{v, f_{2}}^{f}+h_{v, s}^{f}+h_{v, f_{1}}^{f}-\left(n_{2}-1\right) h_{b, s}\right) \frac{q_{c} D}{2 P_{v}}+\left(c_{b}^{S} I_{b}-h_{v, f_{2}}^{f}\right) \frac{n_{2} q_{c}}{2 m_{2}}-\left(h_{v, f_{2}}^{f}+h_{b, s}\right) \frac{n_{2} q_{c}}{2}-\left(m_{1}+\right.$

1) $\left.h_{s, f_{2}} \frac{n_{2} \alpha q_{c} D}{2 m_{1} P_{v}}\right)$

$\frac{\partial^{2} T P_{C-C}}{\partial m_{2}^{2}}=\left(c_{b}^{S} I_{b}-h_{v, f_{2}}^{f}\right) \frac{n_{2} q_{c}}{m_{2}^{3}}<0$ if and only if $h_{v, f_{2}}^{f}>c_{b}^{S} I_{b}$. Otherwise, $m_{2}$ is set equal to 1 .

\section{NC-C Scenario}

\section{a. Proof of concavity with respect to $q_{c}$}

$\frac{\partial^{2} T P_{N C-C}}{\partial q_{c}^{2}}=-\left(S_{s}+S_{v}+n_{1} O_{v}+n_{1} c_{v}^{t}+n_{2} O_{b}+m_{2} c_{b}^{t}\right) \frac{2 D}{n_{2} q_{c}^{3}}<0$

Since the second derivative of the profit function is negative, the total profit is concave in $q_{c}$, which has an optimal solution that maximizes the profit.

\section{b. Proof of concavity with respect to $n_{1}$}

$\frac{\partial^{2} T P_{N C-C}}{\partial n_{1}^{2}}=0$

Since the second derivative of the system's profit is equal to 0 for the NC-C Scenario, and the system's profit (affected by the vendor's profit, see Eq. 7-7) is strictly decreasing as $n_{1}$ increasing, the maximum profit of the system can be obtained by setting $n_{1}$ to the minimum value as much as possible.

\section{c. Proof of concavity with respect to $n_{2}$}

$\frac{\partial^{2} T P_{N C-C}}{\partial n_{2}^{2}}=-\left(S_{s}+S_{v}+n_{1} O_{v}+n_{1} c_{v}^{t}+m_{2} c_{b}^{t}\right) \frac{2 D}{n_{2}^{3} q_{c}}<0$

Since the second derivative of the profit function is negative, the total profit is concave in $n_{2}$, which has an optimal solution that maximizes the profit. 


\section{d. Proof of concavity with respect to $\mathrm{m}_{2}$}

$\frac{\partial^{2} T P_{N C-C}}{\partial m_{2}^{2}}=\left(c_{b}^{S} I_{b}-h_{v, f_{2}}^{f}\right) \frac{n_{2} q_{c}}{m_{2}^{3}}<0$ if and only if $h_{v, f_{2}}^{f}>c_{b}^{S} I_{b}$. Otherwise, $m_{2}=1$.

\section{C-NC Scenario}

a. Proof of concavity with respect to $q_{n c}$

$\frac{\partial^{2} T P_{C-N C}}{\partial q_{c}^{2}}=-\left(S_{s}+S_{v}+n_{1} O_{v}+m_{1} c_{v}^{t}+n_{2} O_{b}+n_{2} c_{b}^{t}\right) \frac{2 D}{n_{2} q_{n c}^{3}}<0$

Since the second derivative of the profit function is negative, the total profit is concave in $q_{n c}$, which has an optimal solution that maximizes the profit.

\section{b. Proof of concavity with respect to $n_{1}$}

$\frac{\partial^{2} T P_{C-N C}}{\partial n_{1}^{2}}=-\left(h_{s, f_{2}}+h_{v, s}^{r}+h_{s, s}+h_{s, f_{1}}\right) \frac{n_{2} \alpha^{2} q_{n c} D}{n_{1}^{3} P_{s}}<0$

Since the second derivative of the profit function is negative, the total profit is concave in $n_{1}$, which has an optimal solution that maximizes the profit.

\section{c. Proof of concavity with respect to $n_{2}$}

$\frac{\partial^{2} T P_{C-N C}}{\partial n_{2}^{2}}=-\left(S_{s}+S_{v}+n_{1} O_{v}+m_{1} c_{v}^{t}\right) \frac{2 D}{n_{2}^{3} q_{n c}}<0$

Since the second derivative of the profit function is negative, the total profit is concave in $n_{2}$, which has an optimal solution that maximizes the profit.

\section{d. Proof of concavity with respect to $m_{1}$}

$\frac{\partial^{2} T P_{C-N C}}{\partial m_{1}^{2}}=-h_{s, f_{2}} \frac{n_{2} \alpha D q_{n c}}{m_{1}^{3} P_{v}}<0$

Since the second derivative of the profit function is negative, the total profit is concave in $m_{1}$, which has an optimal solution that maximizes the profit.

\section{NC-NC Scenario}

\section{a. Proof of concavity with respect to $q_{n c}$}

$\frac{\partial^{2} T P_{N C-N C}}{\partial q_{n c}^{2}}=-\left(S_{s}+S_{v}+n_{1} O_{v}+n_{1} c_{v}^{t}+n_{2} O_{b}+n_{2} c_{b}^{t}\right) \frac{2 D}{n_{2} q_{n c}^{3}}<0$ 
Since the second derivative of the profit function is negative, the total profit is concave in $q_{n c}$, which has an optimal solution that maximizes the profit.

\section{b. Proof of concavity with respect to $n_{1}$}

$\frac{\partial^{2} T P_{N C-N C}}{\partial n_{1}^{2}}=-\left(h_{v, S}^{r}+h_{v, f}^{r}\right) \frac{n_{2} \alpha D q_{n c}}{n_{1}^{3} P_{v}}<0$

Since the second derivative of the profit function is negative, the total profit is concave in $n_{1}$, which has an optimal solution that maximizes the profit.

\section{c. Proof of concavity with respect to $n_{2}$}

$\frac{\partial^{2} T P_{N C-N C}}{\partial n_{2}^{2}}=-\left(S_{s}+S_{v}+n_{1} O_{v}+n_{1} c_{v}^{t}\right) \frac{2 D}{n_{2}^{3} q_{n c}}<0$

Since the second derivative of the profit function is negative, the total profit is concave in $n_{2}$, which has an optimal solution that maximizes the profit.

\section{Appendix 6}

This section provides graphical representations for the four scenarios considered in Chapter 8 .

1. NC-NC Scenario

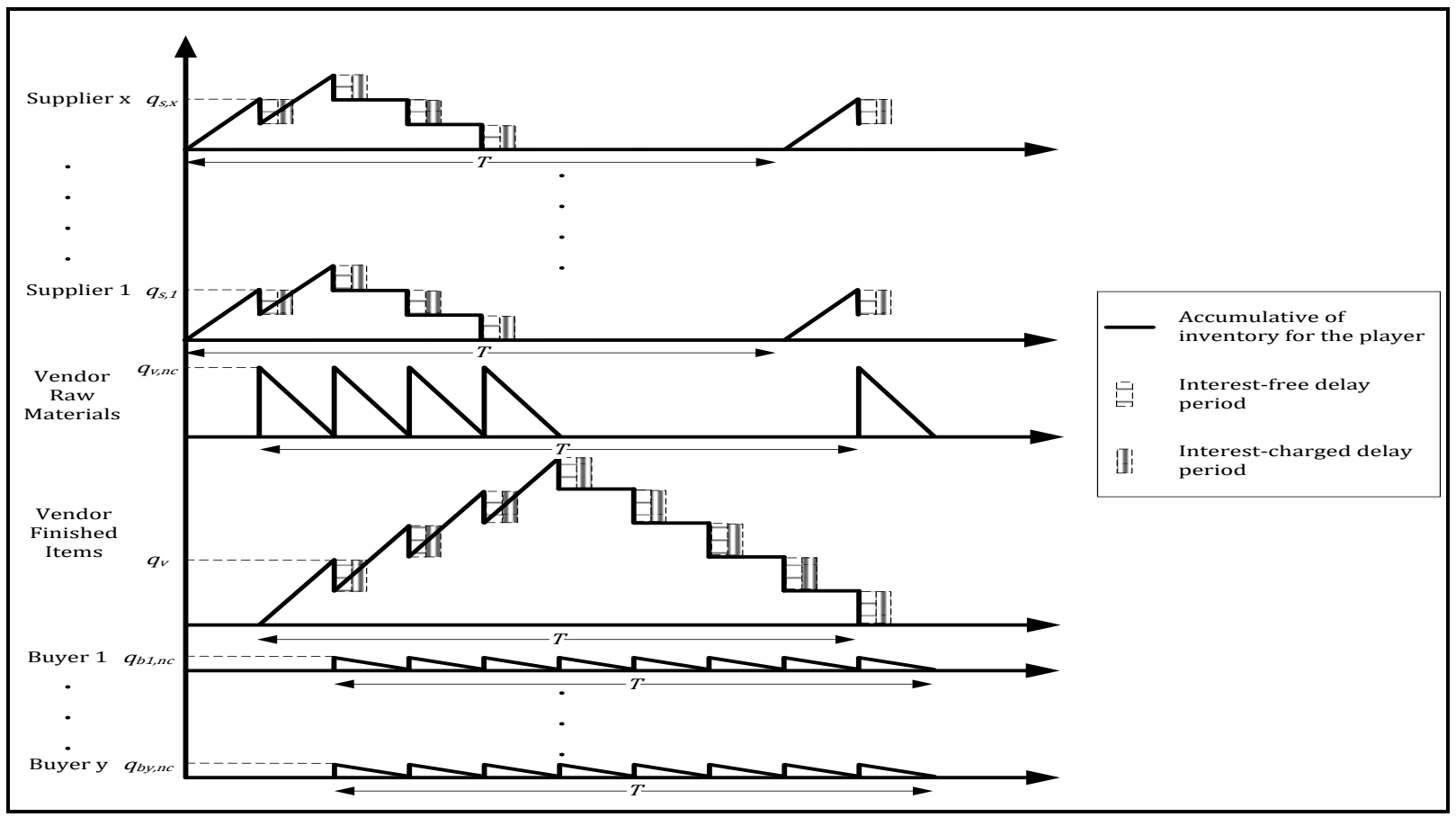

Figure 10-1. Graphical representation for NC-NC Scenario 
2. NC-C Scenario

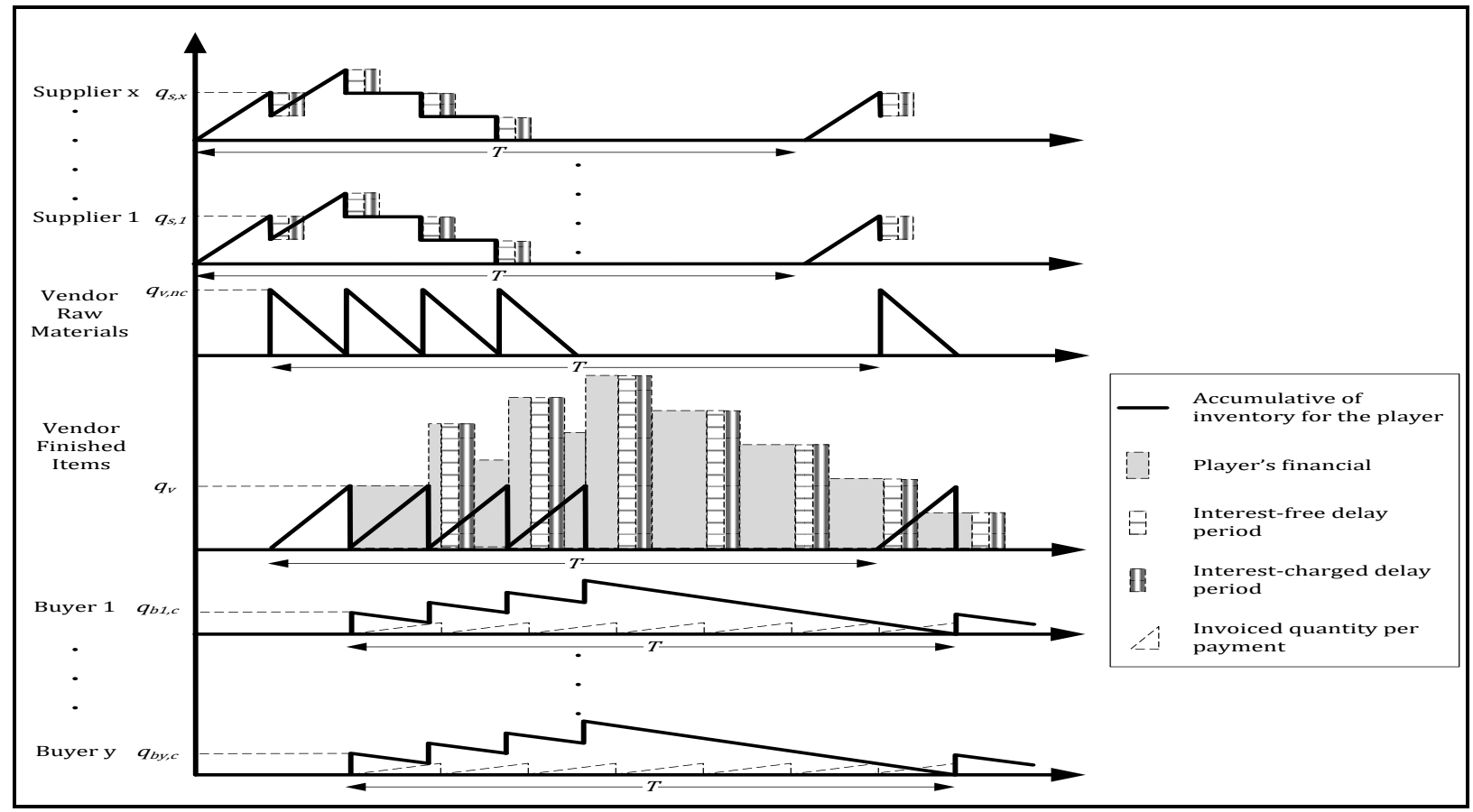

Figure 10-2. Graphical representation for NC-C Scenario

\section{C-NC Scenario}

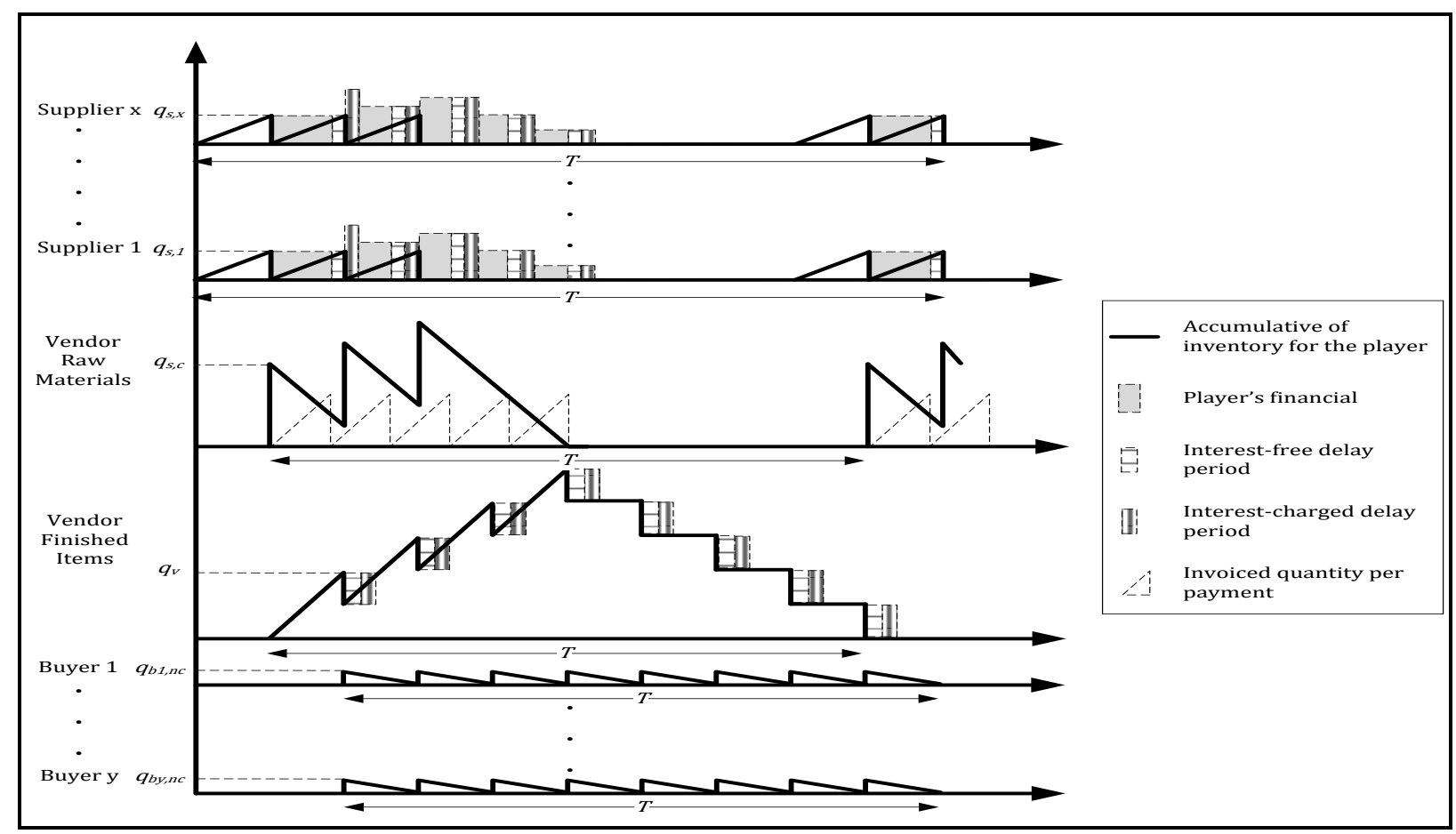

Figure 10-3. Graphical representation for C-NC Scenario 


\section{C-C Scenario}

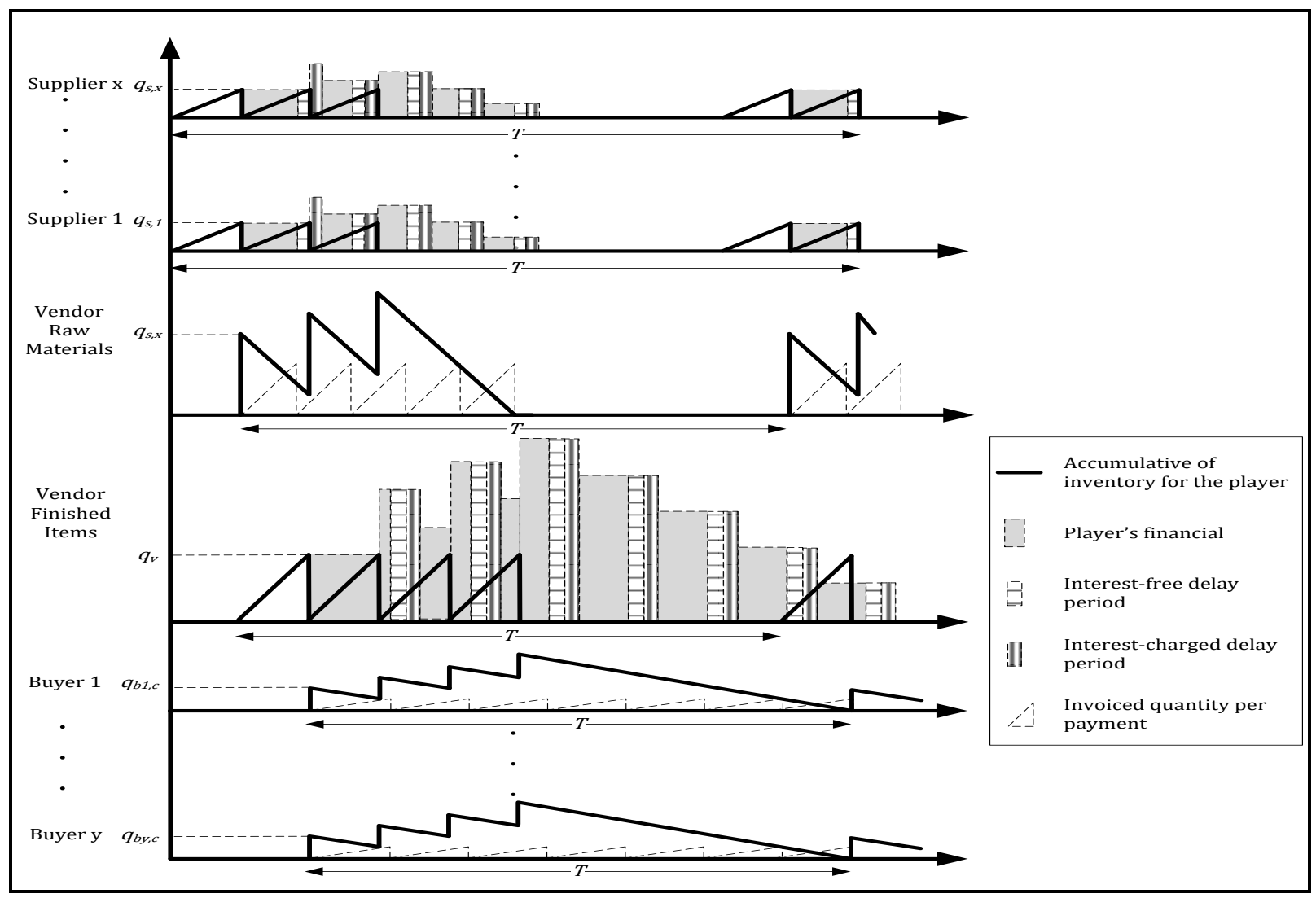

Figure 10-4. Graphical representation for $\mathrm{C}-\mathrm{C}$ Scenario 


\section{References}

Abdul-Jalbar, B., Gutierrez, J. M., \& Sicilia, J. (2007). An integrated inventory model for the single-vendor two-buyer problem. International Journal of Production Economics, 108(1), 246-258.

Aljazzar, S. M., Jaber, M. Y., \& Goyal, S. K. (2016a). Coordination of a two-level supply chain (manufacturer-retailer) with permissible delay in payments. International Journal of Systems Science: Operations \& Logistics, 3(3), 176-188.

Aljazzar, S. M., Jaber, M. Y., \& Moussawi-Haidar, L. (2016b). Coordination of a three-level supply chain (supplier-manufacturer-retailer) with permissible delay in payments. Applied Mathematical Modelling.

Andriolo, A., Battini, D., Grubbström, R. W., Persona, A., \& Sgarbossa, F. (2014). A century of evolution from Harris' s basic lot size model: Survey and research agenda. International Journal of Production Economics, 155, 16-38.

Ballou, R. H., Gilbert, S. M., \& Mukherjee, A. (2000). New managerial challenges from supply chain opportunities. Industrial Marketing Management, 29(1), 7-18.

Banerjee, A. (1986). A joint economic-lot-size model for purchaser and vendor. Decision Sciences, 17(3), 292-311.

Batarfi, R., Jaber, M. Y., \& Zanoni, S. (2016). Dual-channel supply chain: A strategy to maximize profit. Applied Mathematical Modelling, 40(21), 9454-9473.

Battini, D., Grassi, A., Persona, A., \& Sgarbossa, F. (2010a). Consignment stock inventory policy: methodological framework and model. International Journal of Production Research, 48(7), 2055-2079.

Battini, D., Gunasekaran, A., Faccio, M., Persona, A., \& Sgarbossa, F. (2010b). Consignment stock inventory model in an integrated supply chain. International Journal of Production Research, 48(2), 477-500.

Bazan, E., Jaber, M. Y., \& Zanoni, S. (2015). Supply chain models with greenhouse gases emissions, energy usage and different coordination decisions. Applied Mathematical Modelling, 39(17), 5131-5151.

Bazan, E., Jaber, M. Y., Zanoni, S., \& Zavanella, L. E. (2014). Vendor managed inventory (VMI) with consignment stock (CS) agreement for a two-level supply chain with an imperfect production process with/without restoration interruptions. International Journal of Production Economics, 157, 289-301. 
Ben-Daya, M., \& Al-Nassar, A. (2008). An integrated inventory production system in a threelayer supply chain. Production Planning and Control, 19(2), 97-104.

Ben-Daya, M., Hassini, E., Hariga, M., \& AlDurgam, M. M. (2013). Consignment and vendor managed inventory in single-vendor multiple buyers supply chains. International Journal of Production Research, 51(5), 1347-1365.

Braglia, M., \& Zavanella, L. (2003). Modelling an industrial strategy for inventory management in supply chains: The 'Consignment Stock' case. International Journal of Production Research, 41(16), 3793-3808.

Braglia, M., Castellano, D., \& Frosolini, M. (2014). Safety stock management in single vendor-single buyer problem under VMI with consignment stock agreement. International Journal of Production Economics, 154, 16-31.

Bushuev, M. A., Guiffrida, A., Jaber, M. Y., \& Khan, M. (2015). A review of inventory lot sizing review papers. Management Research Review, 38(3), 283-298.

Bylka, S., \& Górny, P. (2015). The consignment stock of inventories in coordinated model with generalized policy. Computers \& Industrial Engineering, 82, 54-64.

Chang, C. T., Teng, J. T., \& Goyal, S. K. (2008). Inventory lot-size models under trade credits: a review. Asia-Pacific Journal of Operational Research, 25(1), 89-112.

Chen, C. H., \& Kan, C. C. (2015). Modified optimum profit model with single supplier and multi-retailers under the consignment policy. International Journal of Management Science and Engineering Management, 10(2), 109-116.

Chen, J. M., Lin, I. C., \& Cheng, H. L. (2010). Channel coordination under consignment and vendor-managed inventory in a distribution system. Transportation Research Part E: Logistics and Transportation Review, 46(6), 831-843.

Chen, L. H., \& Kang, F. S. (2010). Integrated inventory models considering permissible delay in payment and variant pricing strategy. Applied Mathematical Modelling, 34(1), 36-46.

Cheng, T. C. E., \& Wu, Y. N. (2005). The impact of information sharing in a two-level supply chain with multiple retailers. Journal of the Operational Research Society, 56(10), 1159-1165.

Definition of Vendor Managed Inventory. (n.d.). Retrieved April 26, 2013, from http://www.vendormanagedinventory.com/definition.php

Ding, D., \& Chen, J. (2008). Coordinating a three level supply chain with flexible return policies. Omega, 36(5), 865-876.

Fredrickson, J. W. (1986). The strategic decision process and organizational structure. Academy of Management Review, 11(2), 280-297. 
Giri, B. C., \& Bardhan, S. (2015). A vendor-buyer JELS model with stock-dependent demand and consigned inventory under buyer's space constraint. Operational Research, 15(1), 79-93.

Giri, B. C., Bhattacharjee, R., \& Chakraborty, A. (2015). A vendor-buyer integrated inventory system with vendor's capacity constraint. International Journal of Logistics Systems and Management, 21(3), 284-303.

Glock, C. H. (2012). The joint economic lot size problem: A review. International Journal of Production Economics, 135(2), 671-686.

Glock, C. H., Grosse, E. H., \& Ries, J. M. (2014). The lot sizing problem: A tertiary study. International Journal of Production Economics, 155, 39-51.

Goyal, S. K. (1977). An integrated inventory model for a single supplier-single customer problem. The International Journal of Production Research, 15(1), 107-111.

Goyal, S. K. (1988). "A joint economic-lot-size model for purchaser and vendor": a comment. Decision Sciences, 19(1), 236-241.

Grubbström, R. W. (1996). Material requirements planning and manufacturing resource planning. International Encyclopedia of Business and Management, 4, 3400-3420.

Grubbström, R. W., \& Erdem, A. (1999). The EOQ with backlogging derived without derivatives. International Journal of Production Economics, 59(1), 529-530.

Gümüş, M., Jewkes, E. M., \& Bookbinder, J. H. (2008). Impact of consignment inventory and vendor-managed inventory for a two-party supply chain. International Journal of Production Economics, 113(2), 502-517.

Hariga, M. A., \& Al-Ahmari, A. (2013). An integrated retail space allocation and lot sizing models under vendor managed inventory and consignment stock arrangements. Computers \& Industrial Engineering, 64(1), 45-55.

Harland, C. M. (1996). Supply chain management: relationships, chains and networks. British Journal of Management, 7(s1), S63-S80.

Harris, F. W. (1915). What quantity to make at once. The Library of Factory Management, 5, 47-52.

Hill, R. M. (1997). The single-vendor single-buyer integrated production-inventory model with a generalised policy. European Journal of Operational Research, 97(3), 493-499.

Hill, R. M. (1999). The optimal production and shipment policy for the single-vendor singlebuyer integrated production-inventory problem. International Journal of Production Research, 37(11), 2463-2475. 
Hill, R. M., \& Omar, M. (2006). Another look at the single-vendor single-buyer integrated production-inventory problem. International Journal of Production Research, 44(4), 791-800.

Hu, W., \& Li, J. (2012). Disposing the leftovers under the consignment contract with revenue sharing: Retailer vs supplier. Journal of Systems Science and Complexity, 25(2), 262-274.

Huang, Q., \& Chen, J. (2009). A note on "Modelling an industrial strategy for inventory management in supply chains: the 'Consignment Stock' case". International Journal of Production Research, 47(22), 6469-6475.

Inventory. (n.d.). Retrieved May 27, 2013, from https://en.wikipedia.org/wiki/Inventory

Islam, S. S. (2014). Single-vendor single-buyer optimal consignment policy for a seasonal product. Journal of Science and Technology, 59, 66.

J.D. Edwards. (2003). CPFR - Collaborative planning, forecasting, and replenishment (pp.113). Retrieved April 26, 2013, http://www.sccori.com/SCM/COLLABORATIVEPLANNINGFORECASTING.pdf

Jaber, M. Y. (2007). Lot sizing with permissible delay in payments and entropy cost. Computers \& Industrial Engineering, 52(1), 78-88.

Jaber, M. Y., \& Goyal, S. K. (2008). Coordinating a three-level supply chain with multiple suppliers, a vendor and multiple buyers. International Journal of Production Economics, 116(1), 95-103.

Jaber, M. Y., \& Goyal, S. K. (2009). A basic model for co-ordinating a four-level supply chain of a product with a vendor, multiple buyers and tier-1 and tier-2 suppliers. International Journal of Production Research, 47(13), 3691-3704.

Jaber, M. Y., \& Osman, I. H. (2006). Coordinating a two-level supply chain with delay in payments and profit sharing. Computers \& Industrial Engineering, 50(4), 385-400.

Jaber, M. Y., \& Zolfaghari, S. (2008). Quantitative models for centralised supply chain coordination. In: Kordic V, editor. Supply Chains: Theory and Applications. Vienna: I-Tech Educational and Publishing; Ch. 17, pp. 307-338, Vienna, Austria.

Jaber, M. Y., Bonney, M., \& Guiffrida, A. L. (2010). Coordinating a three-level supply chain with learning-based continuous improvement. International Journal of Production Economics, 127(1), 27-38.

Jaber, M. Y., Osman, I. H., \& Guiffrida, A. L. (2006). Coordinating a three-level supply chain with price discounts, price dependent demand, and profit sharing. International Journal of Integrated Supply Management, 2(1), 28-48a. 
Jaber, M. Y., Zanoni, S., \& Zavanella, L. E. (2014a). 'Consignment stock' for a two-level supply chain with entropy cost. European Journal of Industrial Engineering, 8(2), 244-272.

Jaber, M. Y., Zanoni, S., \& Zavanella, L. E. (2014b). A consignment stock coordination scheme for the production, remanufacturing and waste disposal problem. International Journal of Production Research, 52(1), 50-65.

Jones, T. C., \& Riley, D. W. (1985). Using inventory for competitive advantage through supply chain management. International Journal of Physical Distribution \& Logistics Management, 15(5), 16-26.

Khouja, M. (2003). Optimizing inventory decisions in a multi-stage multi-customer supply chain. Transportation Research Part E: Logistics and Transportation Review, 39(3), 193-208.

Krichen, S., Laabidi, A., \& Abdelaziz, F. B. (2011). Single supplier multiple cooperative retailers inventory model with quantity discount and permissible delay in payments. Computers \& Industrial Engineering, 60(1), 164-172.

Krishnan, H., Kapuscinski, R., \& Butz, D. A. (2004). Coordinating contracts for decentralized supply chains with retailer promotional effort. Management Science, 50(1), 48-63.

Lambert, D. M., Stock, J. R., \& Ellram, L. M. (1998). Fundamentals of logistics management. McGraw-Hill/Irwin.

Lee, H. L., \& Whang, S. (2000). Information sharing in a supply chain. International Journal of Manufacturing Technology and Management, 1(1), 79-93.

Lee, H. L., So, K. C., \& Tang, C. S. (2000). The value of information sharing in a two-level supply chain. Management Science, 46(5), 626-643.

Lee, J. Y., \& Cho, R. K. (2014). Contracting for vendor-managed inventory with consignment stock and stockout-cost sharing. International Journal of Production Economics, 151, 158173.

Lee, W. (2005). A joint economic lot size model for raw material ordering, manufacturing setup, and finished goods delivering. Omega, 33(2), 163-174.

Lee, W., \& Wang, S. P. (2008). Managing level of consigned inventory with buyer's warehouse capacity constraint. Production Planning and Control, 19(7), 677-685.

Li, S. X., Huang, Z., \& Ashley, A. (1996). Improving buyer-seller system cooperation through inventory control. International Journal of Production Economics, 43(1), 37-46.

Li, X., \& Wang, Q. (2007). Coordination mechanisms of supply chain systems. European Journal of Operational Research, 179(1), 1-16. 
Lin, Y. J., Ouyang, L. Y., \& Dang, Y. F. (2012). A joint optimal ordering and delivery policy for an integrated supplier-retailer inventory model with trade credit and defective items. Applied Mathematics and Computation, 218(14), 7498-7514.

Liu, L., Sun, Y., \& Yao, J. (2007). An extended analysis of consignment stock policy in the presence of product obsolescence. In 2007 IEEE International Conference on Automation and Logistics, 18 Aug - 21 Aug, pp. 2193-2198, Jinan, China.

Lu, L. (1995). A one-vendor multi-buyer integrated inventory model. European Journal of Operational Research, 81(2), 312-323.

Maddah, B. S., Jaber, M. Y., \& Abboud, N. E. (2004). Periodic review (s, S) inventory model with permissible delay in payments. Journal of the Operational Research Society, 55(2), 147159.

Mandal, P., \& Giri, B. C. (2015). A single--vendor multi-buyer integrated model with stockand price-dependent demand under consignment stock policy. International Journal of Services and Operations Management, 20(2), 228-245.

Mentzer, J. T., DeWitt, W., Keebler, J. S., Min, S., Nix, N. W., Smith, C. D., \& Zacharia, Z. G. (2001). Defining supply chain management. Journal of Business Logistics, 22(2), 1-25.

Molamohamadi, Z., Ismail, N., Leman, Z., \& Zulkifli, N. (2014). Reviewing the literature of inventory models under trade credit contact. Discrete Dynamics in Nature and Society, 2014.

Moussawi-Haidar, L., Dbouk, W., Jaber, M. Y., \& Osman, I. H. (2014). Coordinating a threelevel supply chain with delay in payments and a discounted interest rate. Computers \& Industrial Engineering, 69, 29-42.

Munson, C. L., \& Rosenblatt, M. J. (2001). Coordinating a three-level supply chain with quantity discounts. IIE Transactions, 33(5), 371-384.

Musa, A., \& Sani, B. (2012). Inventory ordering policies of delayed deteriorating items under permissible delay in payments. International Journal of Production Economics, 136(1), 7583.

Oliver, R. K., \& Webber, M. D. (1982). Supply-chain management: logistics catches up with strategy. Outlook, 5(1), 42-47.

Omar, M., \& Supadi, S. S. (2012). Integrated models for shipping a vendor's final production batch to a single buyer under linearly decreasing demand for consignment policy. Sains Malaysiana, 41(3), 367-370.

Persona, A., Grassi, A., \& Catena, M. (2005). Consignment stock of inventories in the presence of obsolescence. International Journal of Production Research, 43(23), 4969-4988. 
Piplani, R., \& Viswanathan, S. (2003). A model for evaluating supplier-owned inventory strategy. International Journal of Production Economics, 81, 565-571.

Richardson, H. (1995). Control your costs then cut them. Transportation and Distribution, 36(12), 94-96.

Ryu, C. (2006). An investigation of impacts of advanced coordination mechanisms on supply chain performance: Consignment, VMI I, VMI II, and CPFR (Doctoral dissertation, State University of New York at Buffalo).

Sadeghi, J., Sadeghi, S., \& Niaki, S. T. A. (2014). Optimizing a hybrid vendor-managed inventory and transportation problem with fuzzy demand: an improved particle swarm optimization algorithm. Information Sciences, 272, 126-144.

Sajadieh, M. S., Thorstenson, A., \& Jokar, M. R. A. (2010). An integrated vendor-buyer model with stock-dependent demand. Transportation Research Part E: Logistics and Transportation Review, 46(6), 963-974.

Salema, M. I. G., Barbosa-Povoa, A. P., \& Novais, A. Q. (2007). An optimization model for the design of a capacitated multi-product reverse logistics network with uncertainty. European Journal of Operational Research, 179(3), 1063-1077.

Sarkar, B. (2012). An EOQ model with delay in payments and time varying deterioration rate. Mathematical and Computer Modelling, 55(3), 367-377.

Sarker, B. R. (2014). Consignment stocking policy models for supply chain systems: A critical review and comparative perspectives. International Journal of Production Economics, 155, $52-67$.

Seifert, D., Seifert, R. W., \& Protopappa-Sieke, M. (2013). A review of trade credit literature: Opportunities for research in operations. European Journal of Operational Research, 231(2), 245-256.

Simatupang, T. M., Wright, A. C., \& Sridharan, R. (2002). The knowledge of coordination for supply chain integration. Business Process Management Journal, 8(3), 289-308.

Srinivas, C., \& Rao, C. S. P. (2010). Optimization of supply chains for single-vendormultibuyer consignment stock policy with genetic algorithm. The International Journal of Advanced Manufacturing Technology, 48(1-4), 407-420.

Srinivas, C., Rao, C. S. P., \& Rao, Y. V. (2008). Consignment stock policy using genetic algorithm for effective inventory management in supply chains. International Journal of Services Operations and Informatics, 3(2), 107-126. 
Stadtler, H. (2008). Supply chain management-an overview. In Supply Chain Management and Advanced Planning (pp. 9-36). Springer Berlin Heidelberg.

Stevens, G. C. (1989). Integrating the supply chain. International Journal of Physical Distribution \& Materials Management, 19(8), 3-8.

Sui, Z., Gosavi, A., \& Lin, L. (2010). A reinforcement learning approach for inventory replenishment in vendor-managed inventory systems with consignment inventory. Engineering Management Journal, 22(4), 44-53.

Surini, Valentina (2011). Inventory Management in Supply Chains: the Three-Level case in Traditional and Consignment Policies (Unpublished master's dissertation, Università degli Studi di Brescia).

Taft, E. W. (1918). The most economical production lot. Iron Age, 101(18), 1410-1412.

Tang, O., Zanoni, S., \& Zavanella, L. (2007). A stochastic single-vendor single-buyer model under a consignment agreement. In Advances in Production Management Systems (pp. 321328). Springer US.

Tirole, J. (2006). The theory of corporate finance. Princeton University Press Princeton USA.

Valentini, G., \& Zavanella, L. (2003). The consignment stock of inventories: industrial case and performance analysis. International Journal of Production Economics, 81, 215-224.

Vendor managed inventory (VMI) - Definition. (n.d.). Retrieved April 26, 2013, from http://www.businessdictionary.com/definition/vendor-managed-inventory-VMI.html

Voluntary Interindustry Commerce Standards (VICS). (2004). Collaborative planning, forecasting and replenishment (CPFR) (pp. 1-24). Retrieved April 26, 2013, from https://www.gs1us.org/DesktopModules/Bring2mind/DMX/Download.aspx?command=core _download\&entryid=492\&language=en-US\&PortalId=0\&TabId=134

Waller, M., Johnson, M. E., \& Davis, T. (1999). Vendor-managed inventory in the retail supply chain. Journal of Business Logistics, 20(1), 183.

Wang, S. P., Lee, W., \& Chang, C. Y. (2012). Modeling the consignment inventory for a deteriorating item while the buyer has warehouse capacity constraint. International Journal of Production Economics, 138(2), 284-292.

Wang, Y., Jiang, L., \& Shen, Z. J. (2004). Channel performance under consignment contract with revenue sharing. Management Science, 50(1), 34-47.

Williams, B. D., \& Tokar, T. (2008). A review of inventory management research in major logistics journals: Themes and future directions. The International Journal of Logistics Management, 19(2), 212-232. 
Wilson, N., \& Summers, B. (2002). Trade credit terms offered by small firms: survey evidence and empirical analysis. Journal of Business Finance \& Accounting, 29(3-4), 317-351.

Wu, J., Ouyang, L. Y., Cárdenas-Barrón, L. E., \& Goyal, S. K. (2014). Optimal credit period and lot size for deteriorating items with expiration dates under two-level trade credit financing. European Journal of Operational Research, 237(3), 898-908.

Yang, P. C., \& Wee, H. M. (2001). An arborescent inventory model in a supply chain system. Production Planning \& Control, 12(8), 728-735.

Yi, H. Z., \& Sarker, B. R. (2014). An operational consignment stock policy under normally distributed demand with controllable lead time and buyer's space limitation. International Journal of Production Research, 52(16), 4853-4875.

Yi, H., \& Sarker, B. R. (2013a). An operational policy for an integrated inventory system under consignment stock policy with controllable lead time and buyers' space limitation. Computers \& Operations Research, 40(11), 2632-2645.

Yi, H., \& Sarker, B. R. (2013b). An optimal consignment stock production and replenishment policy with controllable lead time. International Journal of Production Research, 51(21), 6316-6335.

Zanoni, S., \& Grubbstrom, R. W. (2004). A note on an industrial strategy for stock management in supply chains: modelling and performance evaluation. International Journal of Production Research, 42(20), 4421-4426.

Zanoni, S., \& Jaber, M. Y. (2015). A two-level supply chain with consignment stock agreement and stock-dependent demand. International Journal of Production Research, 53(12), 35613572.

Zanoni, S., Jaber, M. Y., \& Zavanella, L. E. (2012). Vendor managed inventory (VMI) with consignment considering learning and forgetting effects. International Journal of Production Economics, 140(2), 721-730.

Zanoni, S., Mazzoldi, L., \& Jaber, M. Y. (2014). Vendor-managed inventory with consignment stock agreement for single vendor-single buyer under the emission-trading scheme. International Journal of Production Research, 52(1), 20-31.

Zavanella, L., \& Zanoni, S. (2009). A one-vendor multi-buyer integrated production-inventory model: The 'Consignment Stock' case. International Journal of Production Economics, 118(1), 225-232. 